$R \mp$ Conter

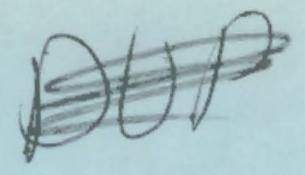

PNL $-365 ?$
NUREG/CR-1845

\title{
FRAPCON-2: A Computer Code for the Calculation of Steady State Thermal-Mechanical Behavior of Oxide Fuel Rods
}

Prepared by G. A. Berna, M. P. Bohn, W. N. Rausch, R. E. Williford, D. D. Lanning EG\&G Idaho, Inc.

Pacific Northwest Laboratory

Prepared for

U.S. Nuclear Regulatory

Commission 


\section{NOTICE}

This report was prepared as an account of work sponsored by an agency of the United States Government. Neither the United States Government nor any agency thereof, or any of their employees, makes any warranty, expressed or implied, or assumes any legal liability or responsibility for any third party's use, or the results of such use, of any information, apparatus product or process disclosed in this report, or represents that its use by such third party would not infringe privately owned rights.

Available from

GPO Sales Program

Division of Technical Information and Document Control

U. S. Nuclear Regulatory Commission

Washington, D. C. 20555

Printed copy price: $\$ 7.00$

and

Nationai Technical Information Service

Springfield, Virginia 22161 
NUREG/CR-1845

R3

\section{FRAPCON-2: A Computer Code for the Calculation of Steady State Thermal-Mechanical Behavior of Oxide Fuel Rods}

Manuscript Completed: December 1980

Date Published: January 1981

Prepared by

G. A. Berna, EG\&G Idaho, Inc.

M. P. Bohn, formerly with EGGG Idaho, Inc., currently with Lawrence Livermore Laboratory

W. N. Rausch, Pacific Northwest Laboratory

R. E. Williford, Pacific Northwest Laboratory

D. D. Lanning, Pacific Northwest Laboratory

EG\&G idaho, inc.

Idaho Falls, ID 83401

Pacific Northwest Laboratory

Richland, WA 99352

\section{Prepared for}

Division of Reactor Safety Research

Office of Nuclear Regulatory Research

U.S. Nuclear Regulatory Commistion

Washington, D.C. 20555

NRC FIN A6050, B2043 



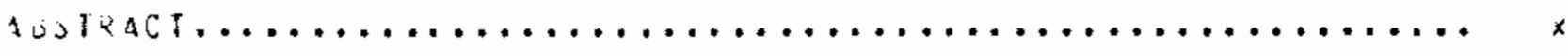

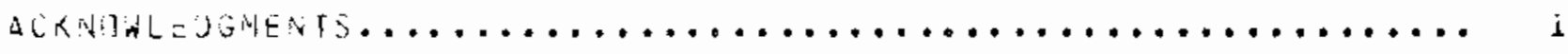

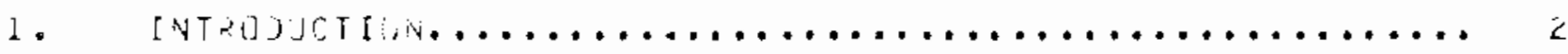

1.1 Jhjegtres of tha riapcon series..............? ?

L.? Limitations of rkapidit-2.................. 4

1.3 Report juting dio zadation to illuer keports......

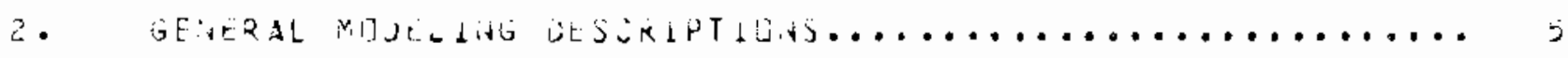

2. FRAHCuitz solution joneme.................. b

2.2 Couplinj of Tnerind and racnanical pojels........

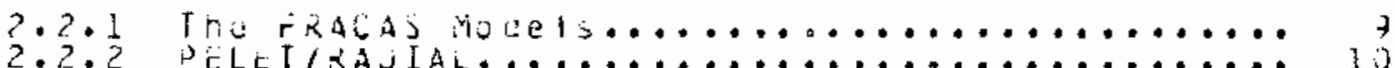

2.3 ruel tiod inerial zesponse................. dy

?.3.1 coolant ionditions.................. 21

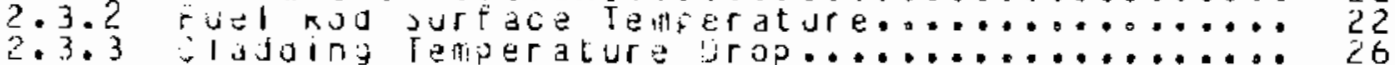

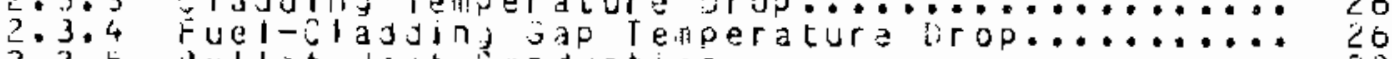

2.3 .5 tel tet tedt

2.3.5. General procedures............ 32

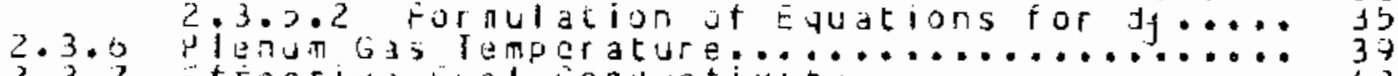

2.3 .7 treccive fual conductivity................ 43

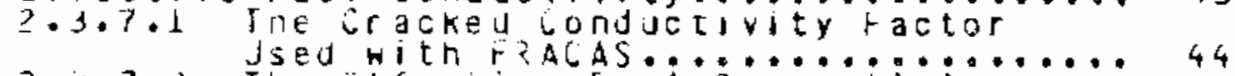

2.3.7.2 The Etfective Fuei canductivity

2.3 .8 stored unergy........................ 46

2.4 Huel rol Machantcal kesponse................ b3

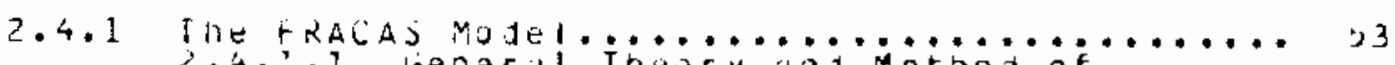

2.4... General Theory and Method of

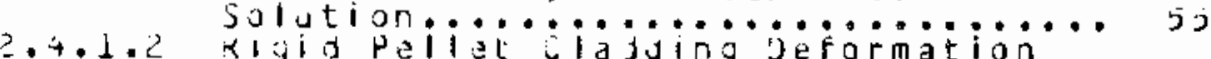

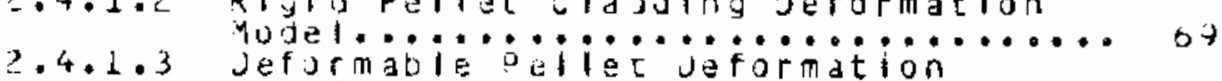

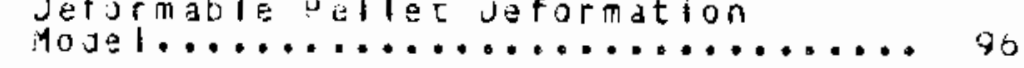




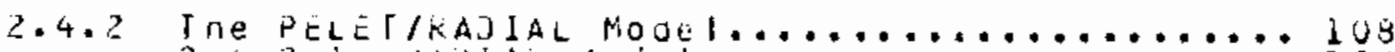

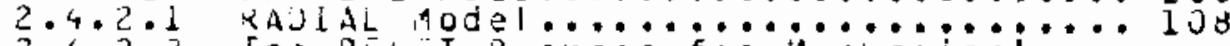
2.4 .2 .2 rne pelet packaje fji

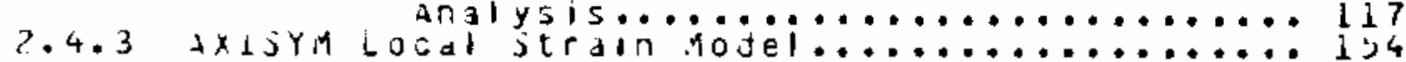

$2 .=$ Fuel koj Internal Gas pressurekesfonse........... I5s

2.j.1 Fuei kou Interndi jas pressure............ 1.5

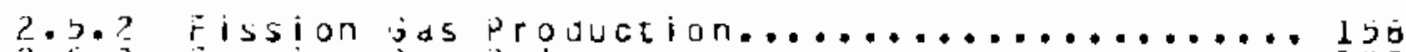

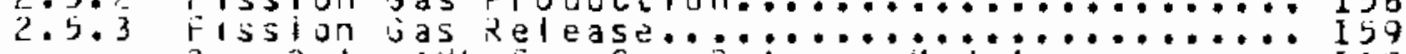

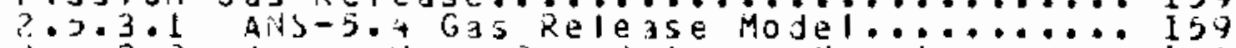

2.2.3.2 jeyer-Hann jas kelkase Mojet....... 164

2.2.3.3 1acjonala-weisman Gas Rel 2 ase

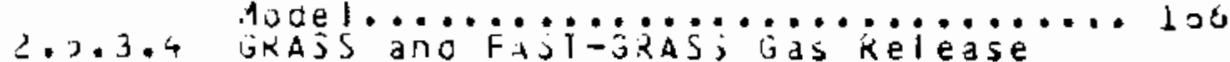

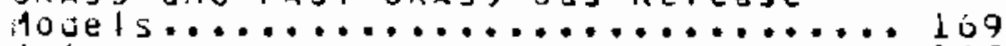

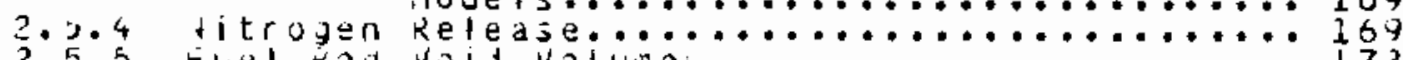

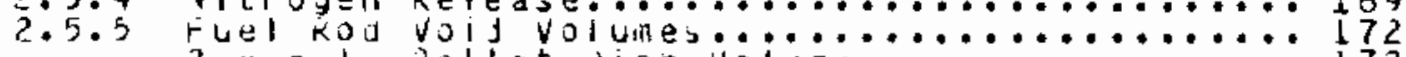

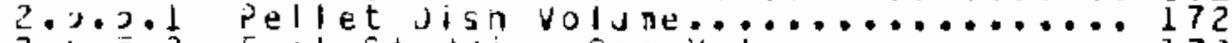

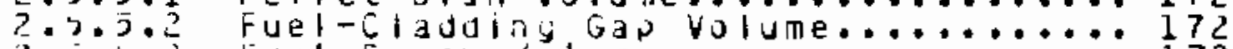

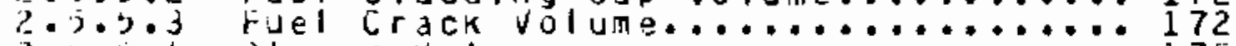

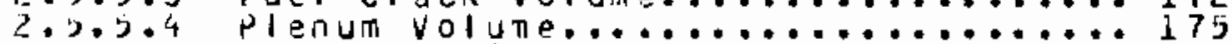

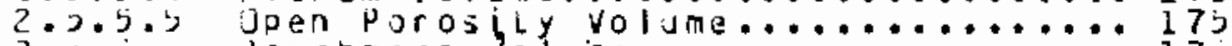

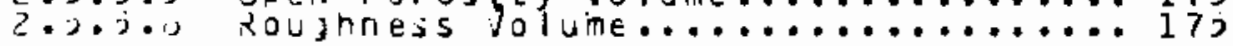

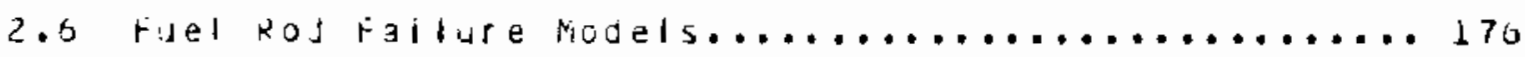

2.6.l Model for cladding Melt................ 177

2.6 .2 houel tor euteclic Melt.................. 177

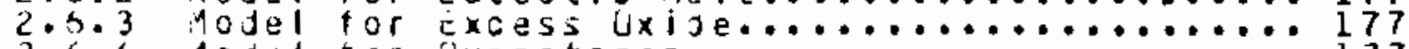

2.5 .4 lojel tor juerstress..................177

2.6 .5 lojel for Erack Growth................. 180

2.6 .6 lodel tor Gverstrain................... 1á

2.7 Uncertainty analysis Option................ 185

2.7 .1 Uncertainty Methodology $2 \ldots \ldots \ldots \ldots \ldots \ldots \ldots \ldots \ldots \ldots$ ldo

3. geitzil cout uescriplin......................... 190

3.1 Cade structure ana solution schene.............. 190

3.1 .1 code structure.......................190

3.1 .2 solution scheme.......................... 192

3.2 Code kesults............................... 198

3.2 .1 Fuel kod kesponse.................... 201

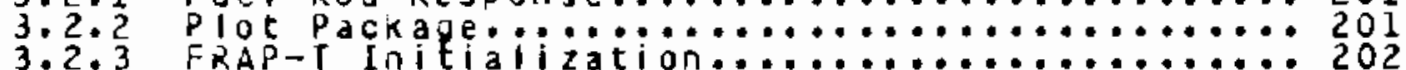


3.3 teatures of FkAPCUN-2.................... 202

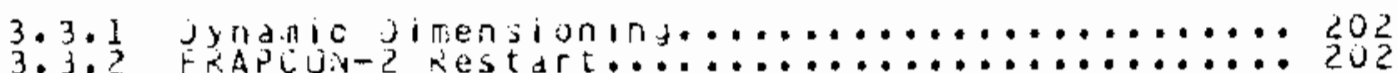

4. REFERENLES................................. 204

appendix A - Input and udtput Descriptlon........... Llu Appendix - taterial properties correlations

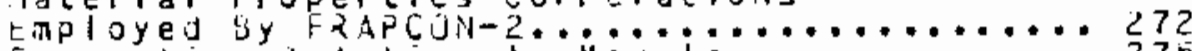
Appendix $c$ - Subroutine kelation to Models............ 275 Appendix - Code Listing and Sample iase Jutput....... 2 dz

NUTE: Appendix D to this report is presented on inicrofiche at achej to the insije back cover.

FIGURES

1. Simplified fKapliat-2 flow chart................. ?

2. Schematic of Typically crackeo fuel rellet.......... 12

1. Flow chart of the ruel and Clauding temperature

calcutation.............................. 19

4. Schematic of tne Fuel Roj Temperature

Distribution............................... 20

2. Thermal Conductivity factor versus Hyarostat ic

Stress lData points loentified by kod Number $\ldots . . . . . .44$

6. Fuel conductivity Multiplier (CrAC) as a Function of Interfacial Pressure and Gas composition.......... jo 
7. Slope of CFac versus loj cressurel iurve of fill

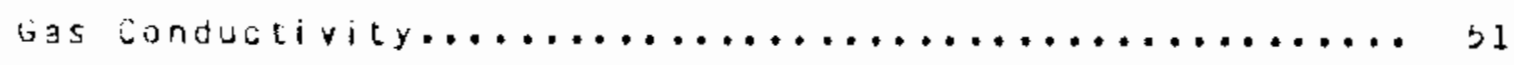

i. Intercept of ifac versus loj (pressure) lurve as a

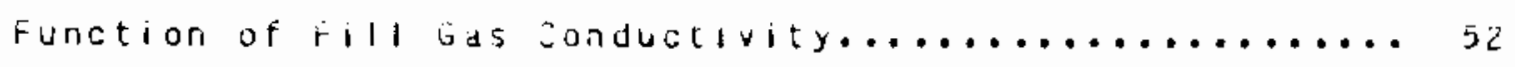

7. Typicat Isotherinal jtress-jitain iurve..................

10. Scnematic of the Metnod of juccessive tiastic

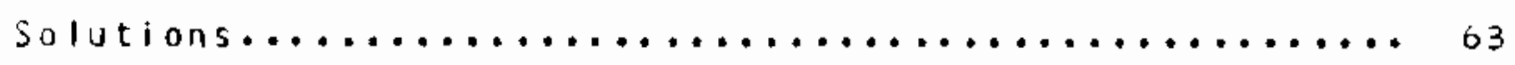

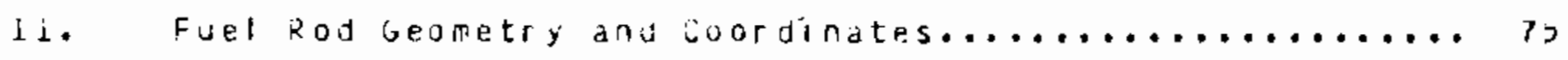

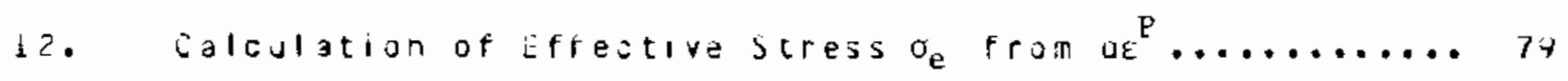

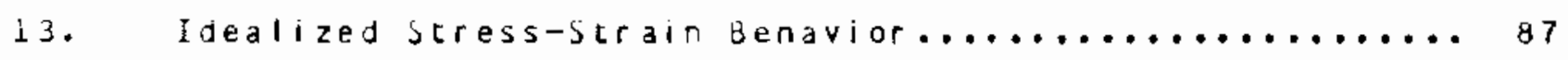

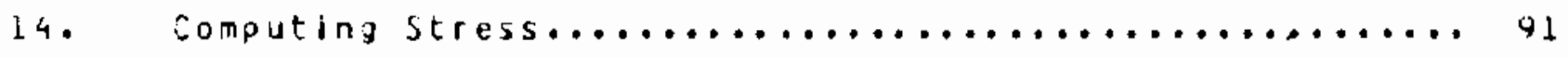

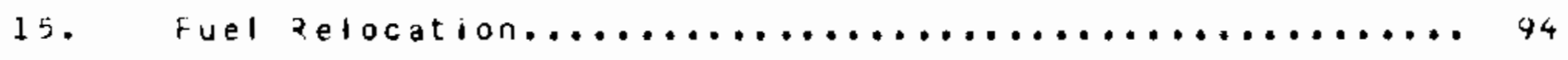

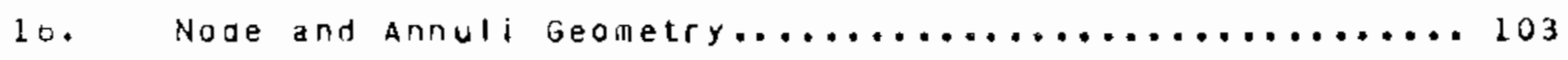

1\%. Convergence Method for Determining Gap Size and

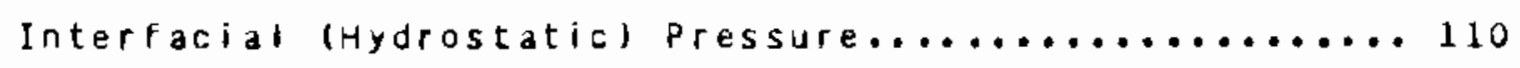

10. Range of Test Rods Examinej in Jerms of free Area 
witnin tne 6 radarh........................... 112

1子. Radial elastic Mojulus as a tunction of ruel arailable Void (bota points identitied oy kod lumoer)..........113

2u. Axial Elastic Modulus as á Funcion of total aviliable Void foata Poinks fuentifiej oy Rog vinoer).......... 114

21. Upper and loner linats for the ruel zadal Mooulus as a Function of iurrent irack Area................. 11

22. Uoper and Lower Limits for the rel bxal hodulus as a Function ut current lotal free Area................ llo

23. Basic Axisymetric king slement used in the stress Analysis Madels...........................................

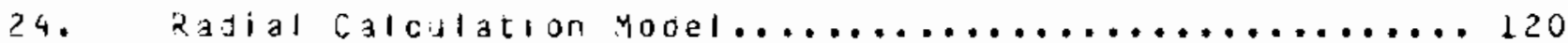

2b. The Axial Mechanical Ansiysis idooel Jsinga Quadrilateral Elenent composed of four Triangular Elenents..................................... 1 i

26. Node and uur Numbertng system for Fuel-iladoing Combined Radial Modef......................... 137

27. Node and Dof Numbering jystem within the Fuel........ 137 
20. Glosal Stiffress tatrax for toe four Elements in figure 27 ljtodal Jiff vumbers are atong the

口utsije) ................................ 138

24. Note and vil duberiny jysten fur tual and cladding

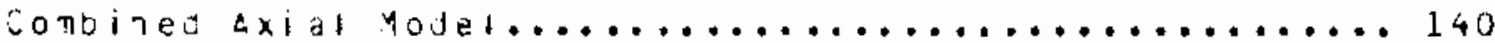

3. Comoarison of Strain Hardening and Time Hardening

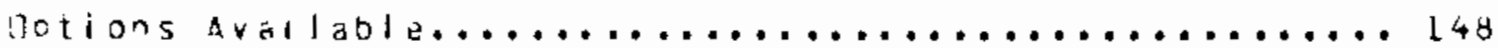

1. Elenents $1,2,3$ and 4 dre Plastic and are brought zack to Prtor jujplastic jtate.................. 1si

32. The plastro strain faken oy alternating plastic

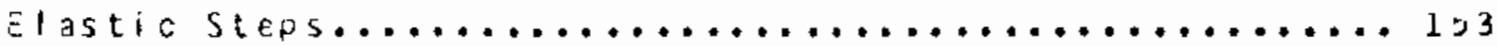

33. Yield stress versus jtrain jsej in Priel calculations

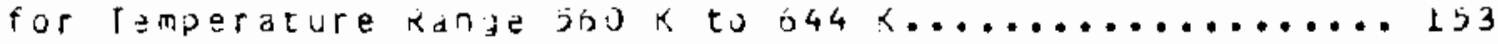

34. disn vaid volume........................... 173

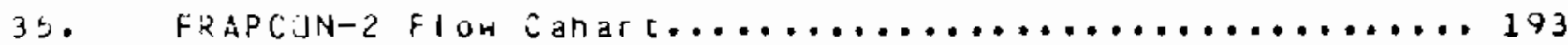

30. Calling Sequence for FRAPGOn-2 Subrojtines........... 194

A-1. Card List Generated oy ECHUL..................... 255

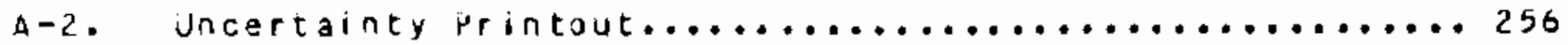




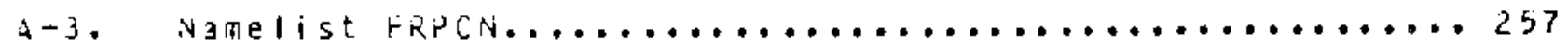

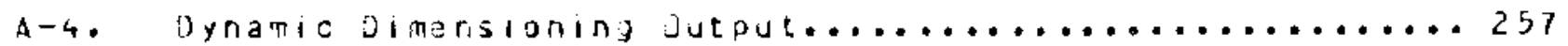

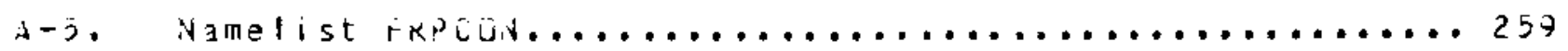

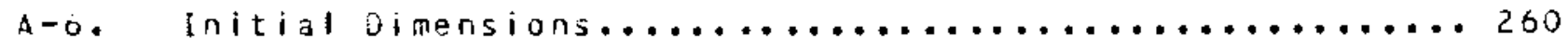

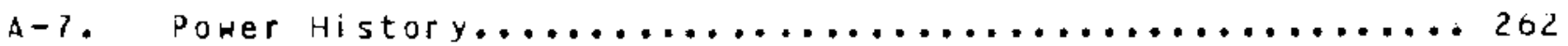

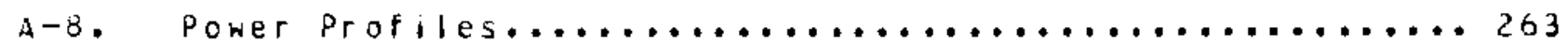

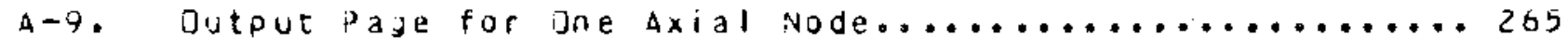

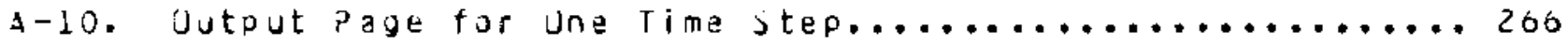

A-11. 4yS-5.4 Rauioactive (jnort-ijed) Fission Gas Release...267

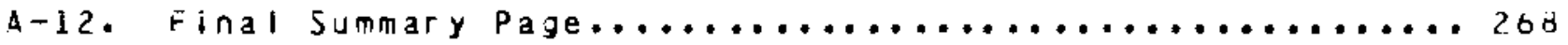

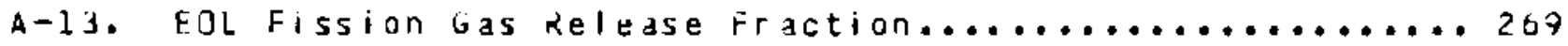

C-1. FRAPCON-2 Sudroutine Relation and Heirarchy.............77

C-2. Relation of jubroutine RADIAL to uther Subroutines ....279 
C-3. Heirarchy ano jequence of subradines witnin the PELt

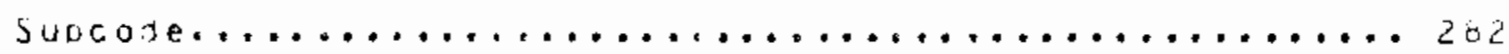

IABLES

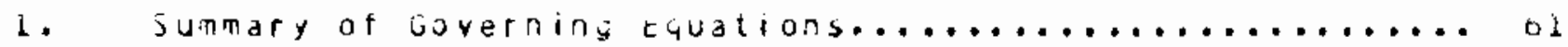

2. Rods Examined for Fuel Hodulus Correlations (All

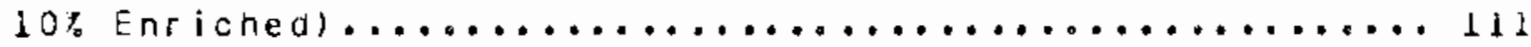

3. Assigned Temperature Bounjaries ana kelease rractions... ló

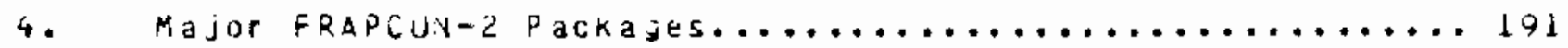

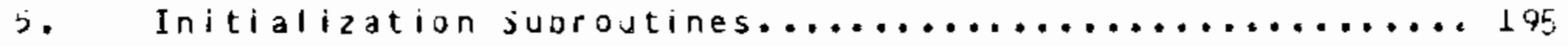

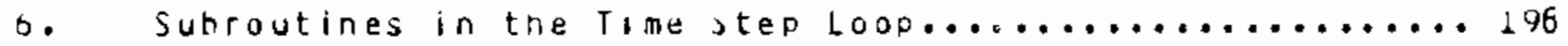

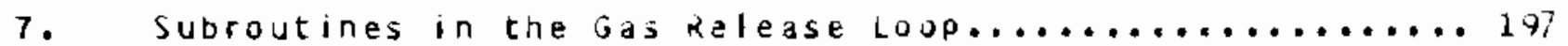

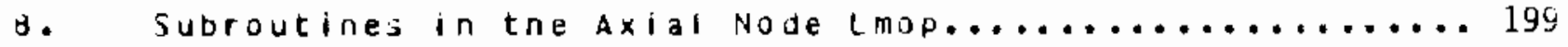

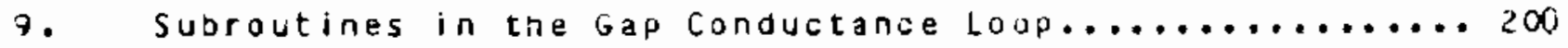

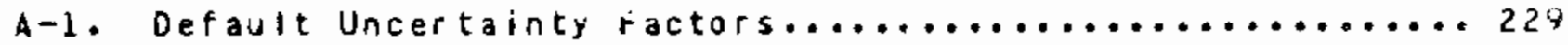

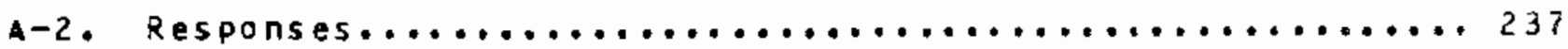




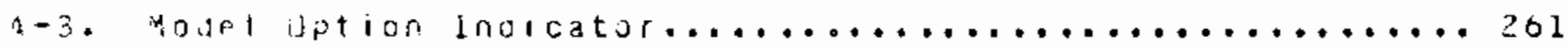

3-i. Materials properties inctuded in Matpku-1

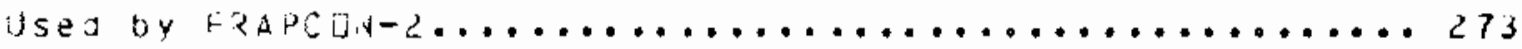


FRAPCON-2 is a FORTRAN IV computer code that calculates the steady state response of light water reactor fuel rods during lonoterm burnup. The code calculates the temperatures pressure, deformation, and failure histories of a fuel rod as functions of t Ime-dependent fuel rod power and coolant boundary conditions. The phenomena modeled by the code include (a) heat conduction through the fuel and cladding, (b) cladding elastic and plastic deformation, (c) fuel-cladding mechanical interaction, (d) fission oas release, (e) fuel rod internal gas pressure, (f) heat transfer between fuel and cladding, (g) cladding oxidation, and (h) heat transfer from clading to coolant. The code contains necessary material properties, water properties, and heat transfer correlations. FRAPCON-2 is programed for use on the CDC Cyber 175 and 176 computers.

The FRAPCON-2 code is designed to generate initial conditions for transient fuel rod analysis by elther the fRAp-To computer code or the ther a al-hydraulic code, RELAPGIMOD7 Version 2. 


\section{ACKNOWLEDGMENTS}

The authors acknowledge Dr. G. P. Marino of the U.S. Nuclear Regulatory Commission for his technical guidance on the models and the modeling processes presented herein. Also we acknowledge Tim Howe, Mitch Cunnincham and Frank Panisko for their careful technical review; Nancy Wildung, Wenoy Bennett, and sue watson for their general technical assistance; and Mary ann Hansen for her efforts in typing this document. Finally, the authors acknowledge Tom Laets for his help in isolating problems in the FRAPCON-2 code whlch were subsequently corrected. 


\section{FRAPCON-2: A COMPUTER CODE FOR THE CALCULATION OF \\ STEADY STATE THERMAL-MECHANICAL BEHAVIOR OF \\ OXIDE FUEL RODS}

\section{INTRODUCTION}

1.1 Dojectives of the FRAPCON Series

The ability to accurately calculate the performance of light water reactor (LWR) fuel rods under long-term burnup conditions is a major objective of the Reactor Safety Research Progran beino conducted by the U.S. Nuclear Regulatory Comission (NRC). To achieve this objective, the NRC has sponsored an extensive program of analytical computer code development, as well as both in-plle and out-of-pile experiments to benchmark and assess the analytical code capabilities. The computer code being developed for the calculation of the long-term burnup response of a single fuel rod is FRAPCON. Inis report describes FRAPCON-2, the second released code of the FRAPCON serjes.

FRAPCON-2 is an analytical tool that calculates LWR fuel rod behavior when power and boundary condition changes are sufficiently slow for the term "steady staten to apply. This includes situations such as long periods at constant power and slow power ramps which are typical of normal power reactor operations. The code calculates the variation with time of all significant fuel rod variables, including fuel and cladding temperatures, cladding hoop strain, cladding oxidation, fuel irradiation swelling, fuel densification, fission gas release, and rod internal gas pressure. In additions the code is destgned to generate initiat conditions for transient 
fuel rod analysis by either FRAP-To, the companion transient fuel roo analysis code, or RELAP4/MOD7, a thermal-hydraulic code for transient analysis of LWR systems.

FRAPCON-2 is linked with the MATPRO ${ }^{3}$ materials properties package. Thus, the user is not required to provide any material property input. The matpko subcode is composed of modular suoroutines that jefine materials properties for tenperatures ranging from room temperatures to temperatures above meltins. Each suoroutine lefines only a single material property. ror example, Yitplo contains suorautines defining fuel tnermal conductivity as a function of fuel temperature and fuel density; fuet tnermal expansion as a function of ruel temperature; and the cladoing stress-strain relation as a tunction of cladding temperature, strain rate, cold work, and fast neutron fluence.

The development of the FRAPCON series is a joint effort of Idaho National Ergineering laboratory (INEl) and Paclfic Northwest Laboratory (PNL) which began with the development of FRAPCON-1. The basis of the FRAPCON-1 code is the FRAP-S $3^{4}$ code developed at INEL with two major changes. First, the code was modified for dynamic dimensioning to increase the number of computer faclities on which the code could be installed. Second, the fRap-S3 temperature subcode was replaced by a more versatile subcode developeo at PNL. The major improvements in FRAPCON-2 with respect to FRAPCON-1 include three advanced mechanics options, FRACAS-II and AXISYM developed at INEL and PELET from GAPCON-THERMAL-3

a. INEL and PNL are operated for the Department of Energy by EGEG Idaho Inc., and Battelie Memorial Institute, respectively.

D. FRAPCON-1, MATPRO-1OA, I daho National. Engineeringlaboratory, EGGG Idaho, Inc., Code Conflguration Control Number HoOj3olB. 
developed at PNL, four additional fission gas release options, bnd an uncertainty analysis option.6

1.2 Limitations of FRAPCON-2

The code has inherent limitations. The rajor limitations are:

1. The thermal models of the code are based on steady state data and equations. Therefore, calculated temperatures will become progressively inaccurate due to this assumption alone as input power histories result in power ramp rates greater than about $0.02 \%$ per secona. Similiarly, the gas release models are based on steady state data and do not reflect release rates expected for rapid power changes.

2. Only small cladding deformations are meaningful. All of the thermal and mechanlcs modeling options assume an axisymetric fuel rod. Large deformations ( $>5 \%$ straln), which tend to become asymetric, will not be traced well by the FRAPCON-2 code. In addition, rapid deformation (greater than about $0.002 \%$ per second) wil not be accurately calculated since a transient temperature calculation would be required to properly include the thermal-mechenlcal reedback.

3. Large power changes are not acceptable to the PELET mechenics wodel. If the PELET option is chosen, power step changes preater than $5 \mathrm{kH} / \mathrm{a}(1.5 \mathrm{k} w / \mathrm{ft})$ per tiat step should not be used. The PELET solution routine is 
incremental and path dependent, and power steps larger than the limit stated above result in an unacceptably large stress-strain increment.

1.3 Report Dutiline and Relation to other Reports

Sections 2. and 3 . of this report deal with the modeling concepts and the code description, respectively. Input instructions, a sample case, materials properties, and subroutine interrelation are discussed in the appendixes. The reader is cautioned that, although the thermal and mechanical models are described separately, they actually are highly interrelated. Section 2.2 is included prior to the detalled modeling descriptions to outline these interrelationships.

This report does not present an assessment of the code performance with respect to in-reactor data. Critical comparisons with experimental data from well-characterized, instrumented test rods will be presented in a separate report describing the developmental assessment of the code. General comparison with a much larper body of data will be presented in a report describing the independent assessment of the FRAPCON-2 code conducted at INEL.

\section{GENERAL MOOELING DESCRIPTIONS}

2.1 FRAPCON-2 Solution Schene

The FRAPCON-2 code iteratively'calculates the interrelated effects of fuel and cladding temperature, rod internal gas pressure, 
fuel and claddino deformation. release of fission product gases, fuel swelling and densification, cladding thermal expansion and irradiation-induced growth, cladding corrosion, and crud deposition as functions of time and fuel rod specific power.

The calculational procedure is illustrated in Flgure 1 , a simplified flowchart of FRAPCON-2. (A detailed flowchart is provided in Section 3). The calculation begins with processing of input data. Next, the initial fuel rod state is determined through a self-initialization calculation. Time is advanced according to the input-specified time step size, a steady state solution is performed, and the new fuel rod state is determined. The new fuel rod state provides the initial state conaltions for the next time step. The calculations are cycled in this manner for the userspecified number of time steps.

The solution for each time step consists of (a) a calculation of the temperature of the fuel and the cladding, (b) a calculation of fuel and cladding deformation, and (c) a calculation of the fission product generation and, void volume, and fuel rod internal gas pressure. When the FRACAS-I mechanics model is chosen, the fuel rod failure probability is also calculated. Each of these calculations is rade in a separate subcode. As is shown in Figure 1, the fuel rod response for each time step is determined by repeated cycling through two nested loops of calculations until the fuel rod temperature, deformation, and internal gas pressure conver oe.

For the FRACAS mechanics models, the fuel temperature and deformation are alternately calculated in the inner loop. On the first cycle through this loop for each time step, the gap conduc- 


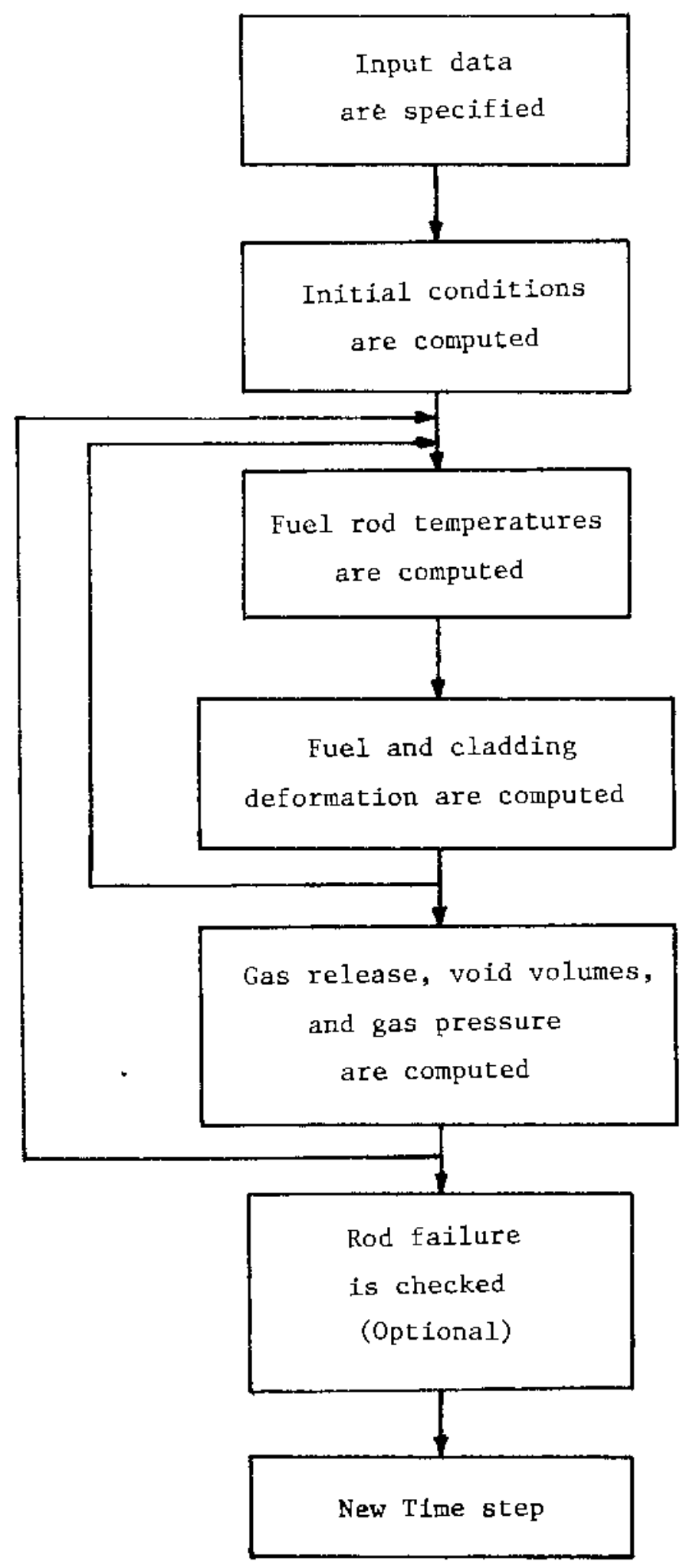

Figure 1. SImplified FRAPCON-2 flow chart. 
tance is computed using the fuel-clading gap size from the previous time step. Then the fuel rod temperature distribution is computed. This temperature distribution feeds the deformation calculation by influencing the fuel and cladding thermal expansions and the cladding stress-strain relation. An updated fuel-cladding gap size is calculated and used in the gap conductance calculation on the next cycle through the inner loop. The cyclic process through the inner loop is repeated until two successive cycles calculate essentially the same temperature distrioution.

The outer loop of calculations is cycled in a manner similar to that of the inner loop, but with the amount of internal gas being determined during each iteration. The calculation alternates between the fuel rod roid volume-gas pressure calculation and the fuet rod temperature-deformation calculation. On the first cycle through the outer loop for each time step, the gas pressure from the previous time step is used. For each cycle through the outer loop, the number of gas moles is calculated and the updated gas pressure computed and feo back to the deformation and temperature calculations (the inner loop). The calculations are cycled until two successive cycles calculate essentialiy the sane gas pressure. When the fuel rod failure option is chosen (see Section 2.61, and after the fuel roo temperatures, deformation and number of gas moles calculated in the two inner loops have converged, fuel rod failure probability is computed. Then a new power time step is begun.

A different solution scheme is used when the PELET mechanics option is chosen. During the first pressure iteration, a simultaneous solution of fuel stress and fuel-cladoing gap size is performed for each axial region prior to the fuel temperature calculation. These calculations are updated during subsequent gas 
pressure calculation iterations. After converoence on fuel rod internal gas pressure, an incremental elastic-plastic deformation calculation is performed for the entire fuel rod. These incremental results are used to update the stress and strain arrays.

\subsection{Coupling of Thermal and Mechanical Models}

The coupling of thermal and mechanical models is such that the packages cannot be used interchangeably. Each thermal-mechanical package and its unifying principles are discussed below starting with the two fRACAS packages and then the PELET/RADIAL package.

2.2.1 The FRACAS Models. The close coupling of the thermal modeling and mechanlcal modeling is the result of the existence of the fuel-cladding gap, and therefore, the space for fuel cracking and relocation. As the fuel temperature increases, the extreme stresses resulting from the large temperature gradients in the fuel cause fuel cracking and relocation to occur. Vold space which is originaliy in the fuel-cladding gap is relocated into the fuel as framents of fuel move outwardy into the fuel-cladding gap. The fuel cracking causes a change in the effective fuel thermal conductivity from the as-fabricated state value.

As the fuel becomes hotter, the fuel expands fliling some of the voids within the fuel. However asperities do not align exactly, thereby causing the fuel diameter to appear laroer and the fuel to interact with the cladding at a lower power than that expected due to normal expansion mechanisms lthermal expansion, swelling, and densification). 
The modeling of the cracked and relocated fuel, both thermally and mechanically, requires accounting for the changed fuel conductivity, changed fuel-cladoing gap size land hence gap conouctancel, ano the changed fuel pellet diameter as the tuel interacts with the cladding. Two models are used to account for these phenomena: Effective fuel thermal conductivity and fuel surface relocation. The effective fuel conductivity model is a correlation wnich frovides a multiplier on the MATPRO fuel conductivity. The fuel surface relocation provides a new fuelciadding gap size fcr use in gap conductance calculations and mechanical interaction calculations. Also considered is the shift of voias from the fuel-cladoing gap into the fuel pellet land the resultant pressure changel and the feedback into the mechanics and thermal calculations.

FRACAS-1 ${ }^{7}$ uses the effective fuel conductivity and the relocated fuel-cladaing gap size for the thermal calculations but does not make use of the fuel surface relocation in the mechanics calculation.

FRACAS-II uses the effective fuel conductivity and the relocateo fuel-clading gap size for the thermal calculations but, unlike FRACAS-I, the refocated fuel surface is used in the mechanics calculations. A model is used to account for time opendent motpressing" of misaligned fuel frayinents.

2.2.2 PELET/RADIAL. The thermal and mechanical responses of a fuel roo to power changes are known to be interdependent. In the conventional viem of a solid pellet stack located concentrically within the cladaing, this mutual dependence can be summarized as fol IOWS: 


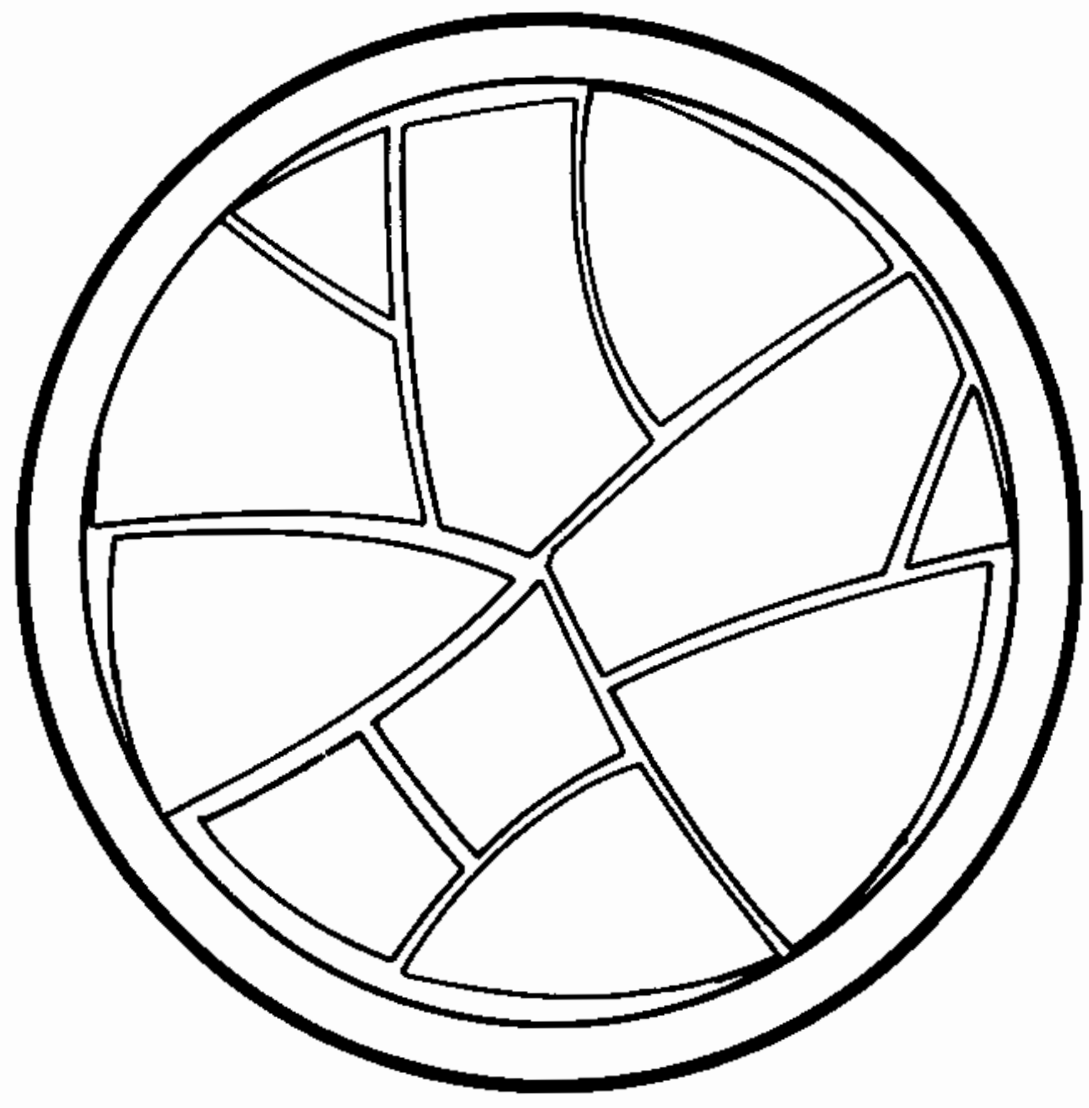

Figure 2. Unifying principles of cracked fuel.

The "free area" is actually cistributed between the fuel cracks and the fuel-cladoing gap. The arount of free area that is occupied by the cracks is aetermined by the lengths and the wioths of the cracks. The lengths of the cracks can be defined by choosing an appropriate crack pattern from postirraciation examation data. The idth of a crack is determined by the interfragment loads (fuel stresses) that cause the cracks to close ouring an increase in power. A relationship between crack width and applied stress was 
1. The symmetric fuel-cladding gap closes with increasing porer due to differential thermal expansion. After fuelcladding contact occurs, further pouer increase results in increasing fuel-cladoing interfacial pressure and an axial ano raoial load on the cladoing.

2. On the other hand, the fuel-cladding gap closure and interfacial pressure changes due the increase in rod power result in an increasing thermal conductance at the fuelcladding interface. This feedback tends to counteract the decrease in fuel intrinsic thermal conductivity with increasing fuel temperature.

In the above view, the "free area" within a given crosssection of the rod resides totaliy in the fuel-ciadding gap. The model is unitied by accounting for how various submodels (thermal expansion, fuel relocation, fuel densification, etc.l change the fued-cladding gap size.

A different view is now proposed which has a more complete set of unifying principles. This new view is based on the fuel pellets being randomly cracked, as is shown in Figure 2 . These cracks are commonly observed in fuel pellets even after very little irraciation. They are caused by the thermal hoop stresses resulting from the steep radial temperature gradients in the fuel. The new modeling of this phenomenon includes a function that relates the crack geometry (wioth) to the fuel stresses. The fuel stresses in turn are related to the fuel temperatures by the differentiat thermal expansions of fuel and cladoino. Thus the crack geometry is relatec to the fuel rod thermal and mechanical responses and to power changes. The concept is briefly discussed below. 
given by Mikic

$1 / 2 \operatorname{erfc}\left(\frac{d}{R \sqrt{2}}\right)=\frac{\sigma}{\sigma+H}$

where

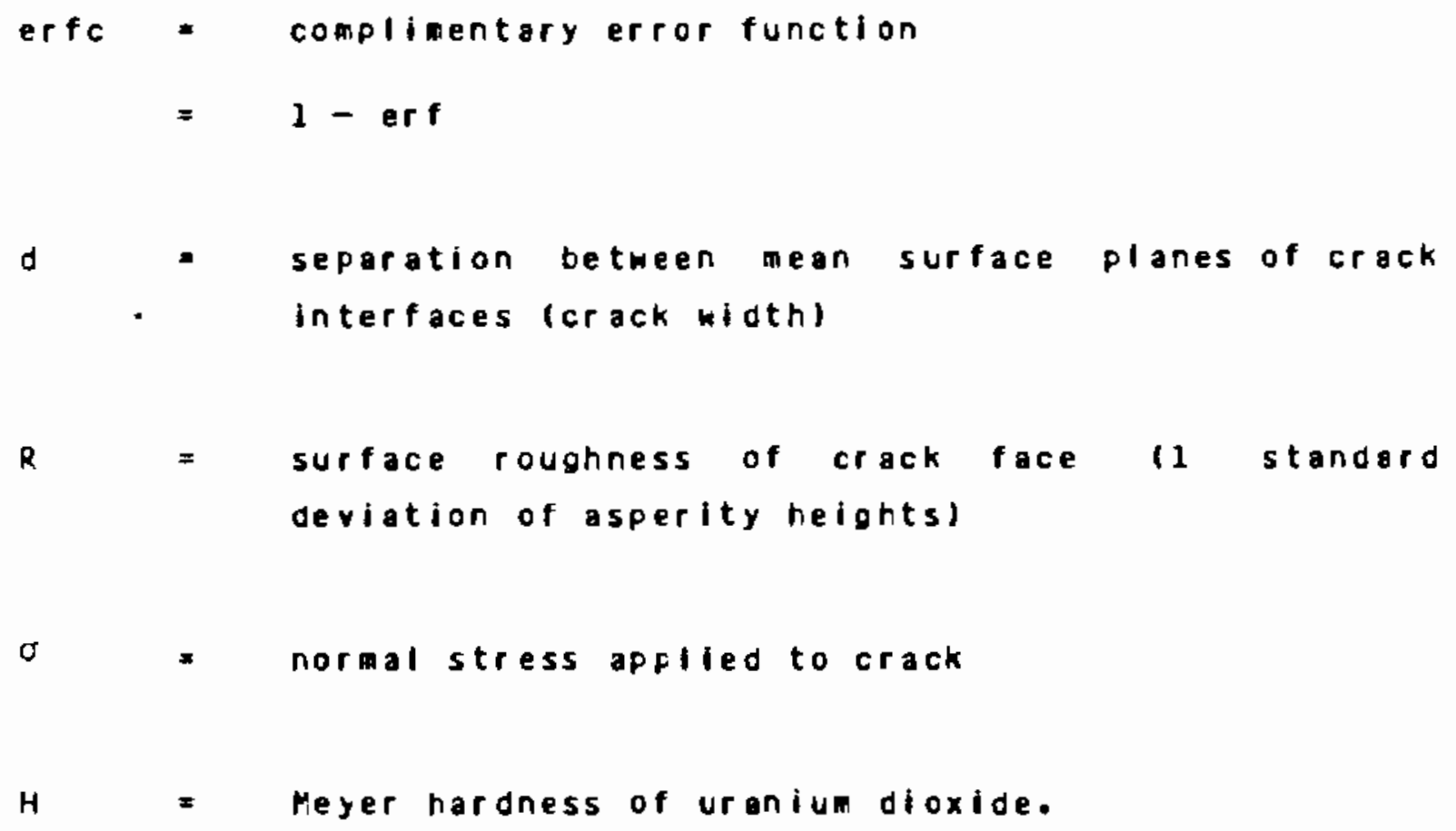

In this application, the surface roughness is actually the roughness which is composed of a combination of the wicroroughess and the surface waviness. If all cracks are assumed to have the same effective roughness and if the fuel is assumed to be in a hydrostatic stress state in the $(r, \theta)$ plane, then all cracks will hove the sane kidth for a specified stress (power) tevel. Crack widh multiplied by total crack length equals total crack area, and the free area occupled by the cracks is thus deterined. 
because the cracks consume some of the free area, they cause the fuel perimeter to increase in length. This is knokn as relocation. However, the fragments become misaligned curing this relocation so that the fuel-cladding gap also has an effective roughness. By virtue of the Mikic surface interaction model, there is always some finite amount of contact between fragments and between fragments and cladding. This occurs because of the error function formulation of the model and provides equilibrium for alt the fragments at all times. At low powers the contact may be very small, but still finite. At higher powers the contact is usually increased substantially. This radial equilibrium condition allows for simultaneous solution of the crack and gap wioths via the Mikic model by equating the fuel hydrostatic stress to the radal stress at the fuel-cladding gap.

There is also axial force equilibrium between the fuel and cladding. Because of the fuel-cladding friction that is induced by constant contact, the fuel and cladding are assumed to be unable to slip axially with respect to each other.

In general, the fuel-cladoing gap sizes lfuel-cladoing surface separations) calculated by this model are substantialiy less than those calculated by the solid cylinder model. About half of the free area is usually consumed by cracks in the fuel. The degradation of the fuel thermal conductivity is dependent on the voids (cracks) that exist within the fuel. Thus, the primary feedback mechanism between fuel temperatures ano fuel-cladoing thermal expansion is crack closure and its effect on fuel thermal conductivity, rather than fuel-cladding gap closure and its effect on gap conductance. 
The axiomatic foundations of the approach are now summarized.

1. The fuel is cracked and the free area is distributed between tuel cracks ano the fuei-cladding gap.

2. The amount of tree area occupied by the cracks is determined by crack geometry (length and widh) and the stress level. Crack width is related to the fuel stress by the Mikic surface interaction model.

3. The fuel stress state in the (r, $)^{\prime}$ plane is assunied to be hyorostatic, and all cracks within the fuel have equal roughness. Thus, all crack wioths are assumed equal. (A separate roughness is assigned to the fuel-claoding gapl.

4. The fuel fragments and cladding are alkays in radial and axial equilitrium. Thus, there is always fuel-cladoing contact and never axial slippage between fuel and cladding.

5. The primary thermal-mechanical feedback is due to crack width effects on the effective fuel thermal conductivity, rather than by the fuel-clading gap closure effects on cap conductance.

The above principles have been used to reinterpret in-reactor power, fuel temperature and cladding elongation data. ${ }^{9}$ From this reinterpretation came numerical estimates (empirical correlations) for the fuel conductivity and effective fuel elastic moduli as a function of the estimated free area partition and hydrostatic stress 
state of the fuel. The procedure used for this reinterpretation will now be reviewed to explain both how the correlations were deriveo and how they are used in PELET/RADIAL.

The data used came primarily from Halden Reactor Tests IFA-432 10 and IfA-513.11 For those tests the following information for a large range of fuel-clading gap sizes ano fill gas composition was arailable:

1. Accurate steady state power versus temperature data

2. Transient temperature versus time data lylelding an estimate of total thermat resistance apportioned to fuel and gap thermal resistance) ${ }^{a}$

3. Cladding elongation as a function of time and power.

Gap and crack roughness (assumed proportionall were found by trial as a function of the as-fabricated fuel-cladding gap size so that the calculated conductances ritched those deduced from transient data. These then became fixed values in the data analysis, as they are now in the code. A fixed value of total crack length ( 3.5 pellet diameters) was also chosen, based on inspection of many photographs of irradiated peliets. With these parameters fixed, the Mikic model could be used to afrive simultaneously at

a. Total thermal resistance is defined as the differencebetween fuei centeríne and coolant temperatures divided by the local inear heat rate. Reference 12 provides an expanded discussion of thermal resistance and the use of transient temperature data. 
fuel-cladoing yap size, crack size, and hydrostatic stress in the fuel, given fuel centerline temperature and fuel power. The incremental changes in deduced fuel strain and stress from one power level to the next were used to estimate an effective radial fuel elastic modulus. Similarly, iascemental changes in cladding elongation were used to estimate an effective elastic modulus. The moduli were correlated to the estimated free area while the deduced change (aegradation) in the fuel thermal conductivity was correlated to the fuel hyorostatic stress.

Within PELET/RAOIAL, the same principles apply in the sense that the thermal-mechanical state in the current time step is estimated from the previous time step plus the incremental change in power and temperature. The thermal and mechanical models are coupled in the following manner:

1. Given the current power and the previous cladding radius, the amount and partition of pree area within the fuel rod is found for each axial node, together mith the corresfonding hydrostatic stress.

2. From item 1, fuel-cladding gap size and interfacial pressure are immediately arailable. Both of these variables go into the gap conductance calculation, and the fuet-cladding interfacial pressure feeds the calculation of radial and axial cladding stress and strain via the finite element formulation in PELET.

3. The thermal conductivity degradation is estimated from an empirical correlation involving hydrostatic stress and 
fill gas composition. This feeds to the fuel temperature, and hence, to the fission gas release calculations.

4. The raaial ana axial fuel elastic moduli are estimated from emfirical correlations with the free area. These feed directly to the calculation of cladoing stress and strain at the end of the time step.

Within the fELET/RAEIAL package, fuel-cladding gap size and interfacial pressure are oot varied during the iterations that determine the temperature orop across the fuel-cladding $g a p$, and the computer time normally spent performing these calculations has been transferred to determining the hyarostatic stress state for the crackeo fuel. Ihis stress state determination lies within the gas iteration loop and is updated due to temperature changes resulting from gas release. The mechanical calculation of incremental cladaing deformation lies outside both iteration loops and is done at the end of the time step.

In summary, the thermal and mechanical models are intimately interrelated. But by nandling that interrelationship on an incremental basis, computer running time needed is minimized.

\subsection{Fuel Rod Thermal Response}

The temperature distribution throughout the fuel and the cladding is calculated at each axial node. A simplified flowchart of the temperature aistribution solution is shown in Figure 3. A schematic drawing of the temperature distribution at an arbitrary axial node is shown in figure 4. 


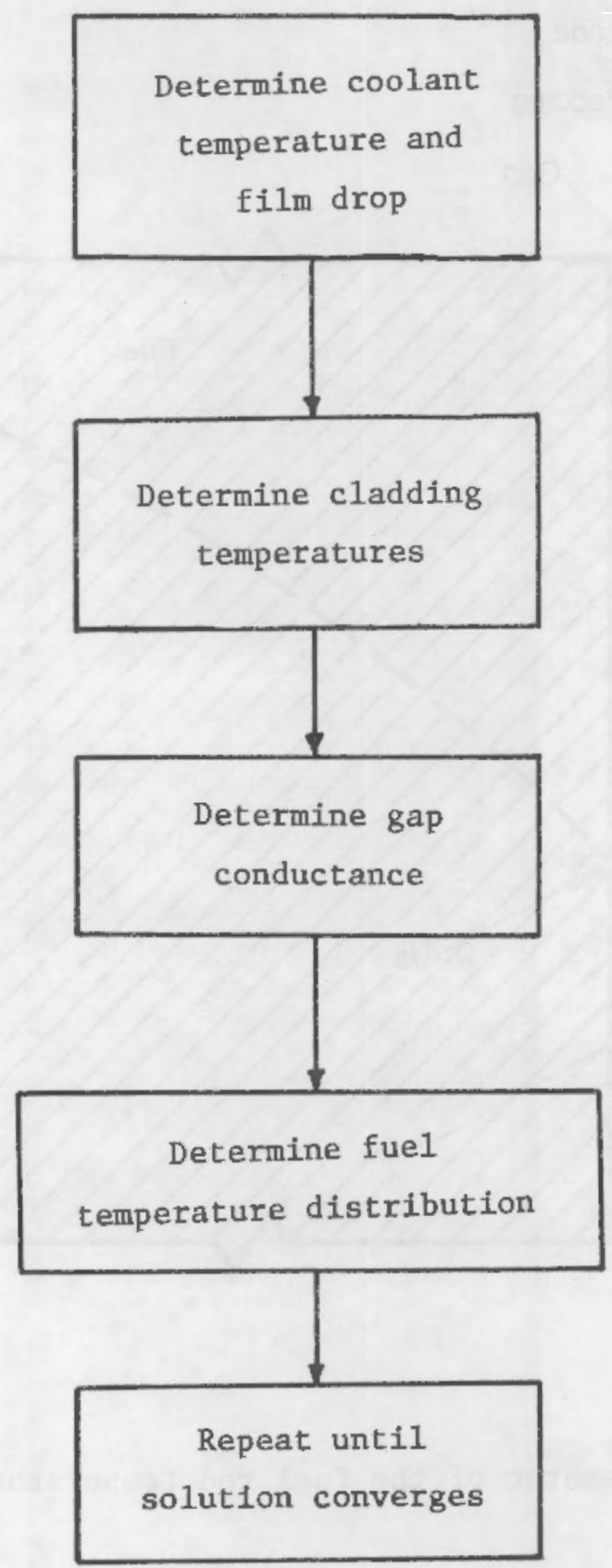

Figure 3. Flow chart of the fuel and cladding temperature calculation. 


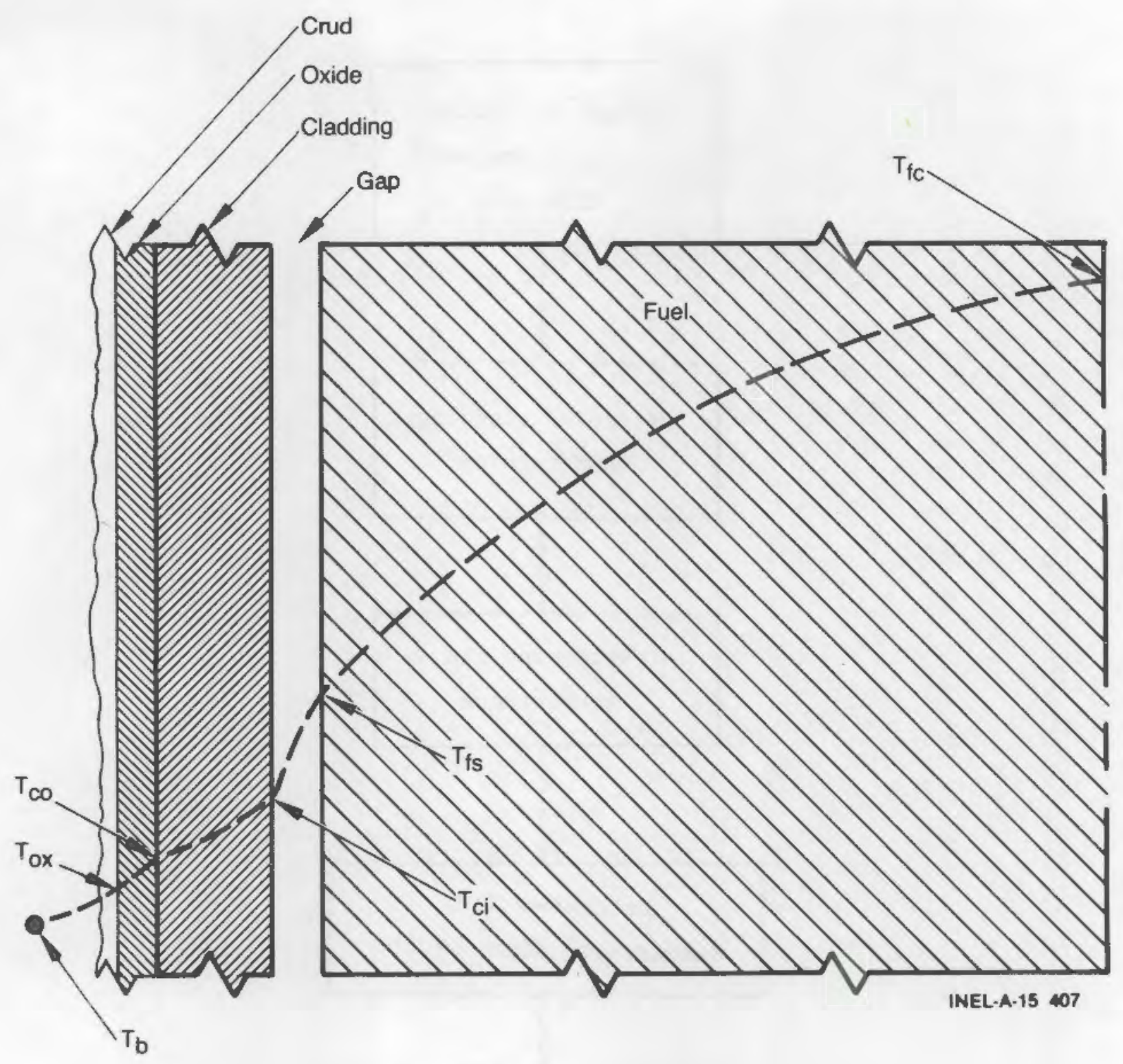

Figure 4. Schematic of the fuel rod temperature distribution.

The models used in the fuel rod temperature calculations assume a cylindrical fuel rod surrounded by coolant. User supplied boundary conditions (coolant inlet temperature, coolant channel equivalent heated diameter, and the coolant rass f $(u x)$ and the user supplied axial linear heat generation rate are used to calculate the 
coolant bulk temperature, $T_{b}$, using a single channel coolant enthalpy rise nocel. A film temperature rise, $T_{f}$ is then calculated from the coolant to the surface of the fuel rod through any cruo layer which may exist. The cladoing inside surface temperature, $r_{c i}$, is found by calculating the temperature rise across the zirconium oxide and the cladoing using fourier's Law. The temperature rise to the fuel surface is determined from an annular gap conductance model, thereby establishing the fuel surtace temperature, $T_{f s}$. Finally, the temperature distribution in the fuel is calculated, accounting for fuel cracking effects using the fuel surface temperature and assumed symetry at the centerline as boundary conoitions.

Tre models used in the temperature calculations involve a number of assumptions and limitations. The most important are:

1. No heat conduction in the axial direction

2. No neat conduction in the azimuthal direction

3. Constant boundary conditions during each time step

4. Steady state

5. The fuel rod is a right circular cylinder surrounded by water coolant.

2.3.1 Coolant Conditions. FRAPCON-2 calculates bulk coolant temperatures assuming a single, closed coolant channel according to 
$T_{b}(z)=T_{i n}+\int_{0}^{z}\left[\frac{4 \dot{q}^{\prime \prime}(z)}{C_{p} G_{e}}\right] d z$

where

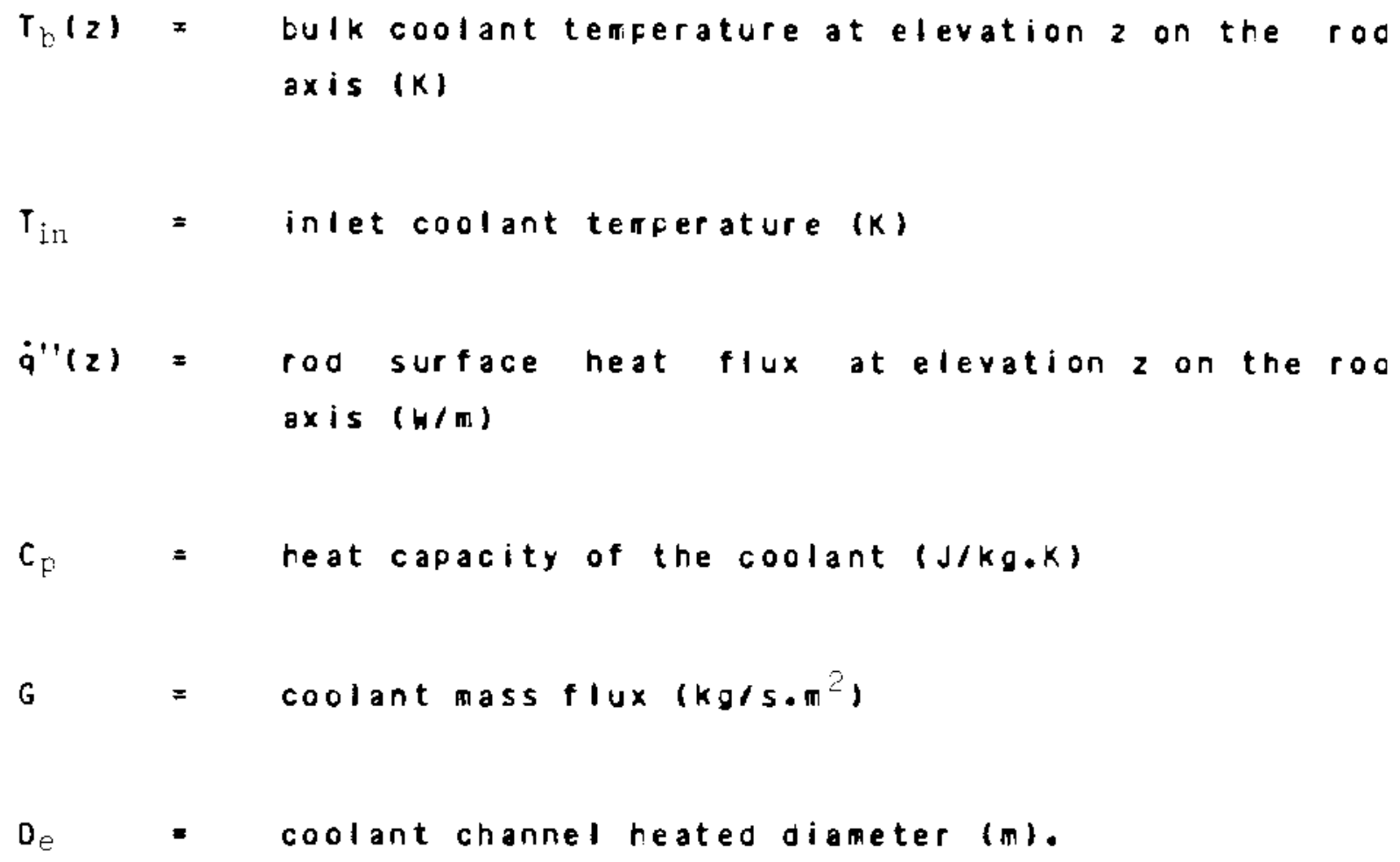

2.3.2 Fuel Rod Surface Temperature. The cladoing surface temperature at axial elevation $z$ is taken as the minimum value of

$$
\begin{aligned}
& T_{w}(z)=T_{b}(z)+\Delta T_{E}(z)+\Delta T_{c}(z) \\
& T_{w}(z)=T_{s a t}+\Delta T_{J L}
\end{aligned}
$$


where

$$
\begin{aligned}
T_{w}(z)= & \text { roo surface teiperature at elevation } z \text { on the rod } \\
& \text { axis }(K)
\end{aligned}
$$

$\begin{aligned} \Delta T_{f}(z)= & \text { forcea convection film temperature orcp at } \\ & \text { elevation } z \text { on the rod axis (K) }\end{aligned}$

$\Delta T_{c}(z)=$ crud temperature drop at elevation $z$ on the roo axis $(K)$

$T_{\text {sat }}=$ coolant saturation temperature $(K)$

$\Delta T_{i L}=$ nucleate boling temperature crop at elevation $z$ on the roo axis $(K)$.

The choice of the minimum value is a simple means of deciding whether heat is transferred trom the cladoing surface to the coolant by forced convection or nucleate bolling. It also provides a smooth numerical transition from forced convection to nucleate boiling thereby avoiding convergence problems. For forced convection heat transfer, the temperature drop across the coolant film layer at the rod surface is based on

$$
\Delta T_{E}(z)=\dot{q}^{\prime \prime}(z) / h_{E}
$$

where $h_{f}$ is the Dittus-Boelterl3 film conductance given by 
$n_{E}=\left(0.023 k / D_{e}\right) \quad R_{e}^{0.8} P_{r}^{0.4}$

$(t)$

where

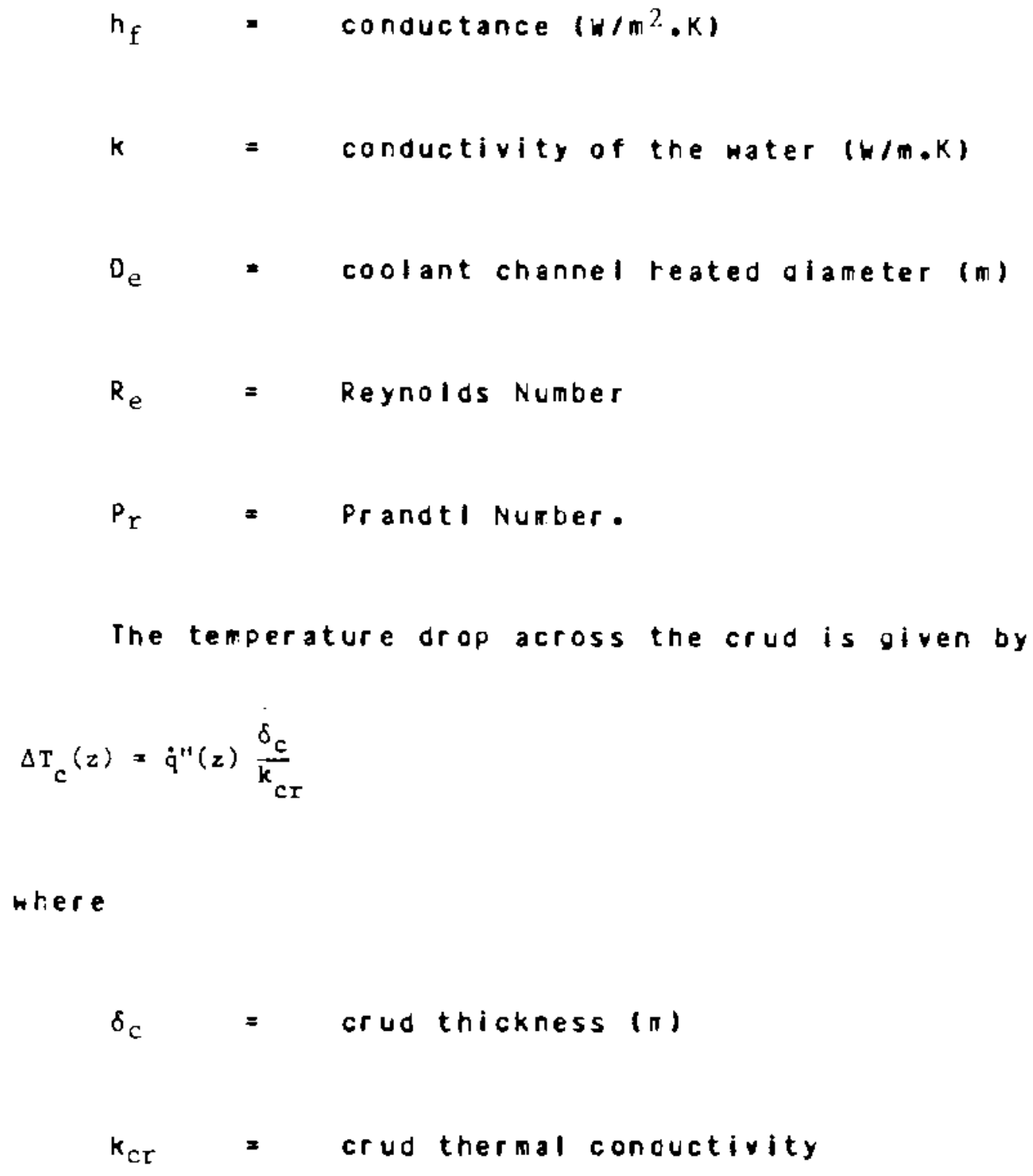




\section{$=0.8648(H / \pi \cdot K)$}

For nucleate bolling heat transfer, the temperature orop across the coolant film layer at the rod surface is based on the JensLetes ${ }^{14}$ formulation

$\Delta \mathrm{T}_{\mathrm{JL}}(\mathrm{z})=60\left[\dot{q}^{\prime \prime}(\mathrm{z}) /\left.10^{6}\right|^{0.25} / \mathrm{e}^{\left(\mathrm{P} / 6.2 \times 10^{6}\right)}\right.$

where

P = system bulk coolant pressure (Pa).

No additional temperature drop from the cladoing surface to the coolant is assumeo to occur due to crua deposition men nucleate boiling exists. The coolant is assumed to voil thrcugh the crud b I anket.

The temperature drop across the zirconium oxloe layer at elevation $z$ on the rod axis is deterineo by

$$
\Delta T_{0}(z)=\frac{\dot{q}^{\prime \prime}(z) \delta_{\Omega}(z)}{k_{0}}
$$

where

$$
\begin{aligned}
\Delta T_{0}(z)= & \text { oxide temperature drop at elevation } z \text { on the roo } \\
& \text { axis (K) }
\end{aligned}
$$




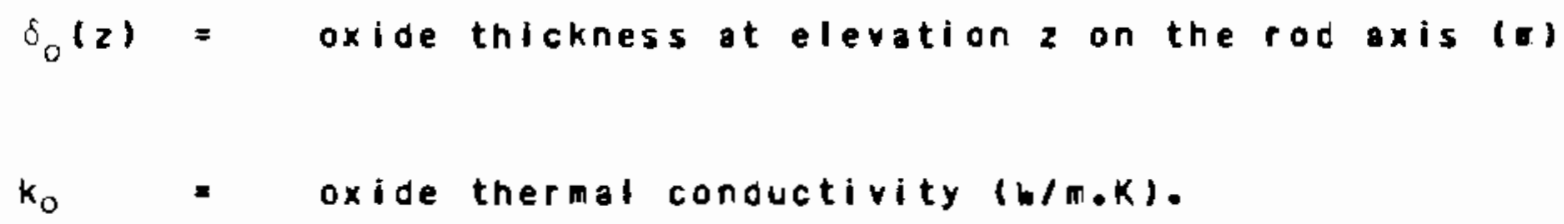

2.3.3 Cladding Temperature crop. The cladding temperature drop for each axial location is calculated according to the following expression for steaoy state heat transfer through a cylinder with uniform thermat conouctivity:

$$
\Delta \mathrm{T}_{c l \mathrm{ad}}=\dot{q}^{\prime \prime}(z) r_{0} \ln \left(\mathrm{r}_{\mathrm{o}} / \mathrm{r}_{\mathrm{i}}\right) / \mathrm{k}_{c l a d}
$$

where

$$
\begin{aligned}
& \Delta T_{c l a d}=\text { clading tenperature arop }(K) \\
& \text { ro cladding outside radius (m) } \\
& r_{i} \quad \text { clading inside radius }(m) \\
& k_{\text {clad }} x \text { temperature and material dependent thernal } \\
& \text { conductivity of the cladding (w/m.k). }
\end{aligned}
$$

2.3.4 Fuel-CIadding Gap Temperature Drop. The fuel-cladding gap conductance is the sum of three components: the conductance due to radiation, the conduction through the oas, ano the conouction through regions of solid-solid contact. The equations and aodels for each of these components are oiscussed below. 
Radiation Heat Transfer--The net radiant heat transfer of heat from the fuel to the cladoing is the infinite-cylinder oray body form as derlved by Kreith ${ }^{15}$ and others:

Net surface heat $\operatorname{flux}(\mathrm{S} . \mathrm{H} . \mathrm{F})=\sigma \mathrm{F}\left[\mathrm{T}_{\mathrm{fs}}^{4}-\mathrm{T}_{\mathrm{ci}}^{4}\right]$

wrere

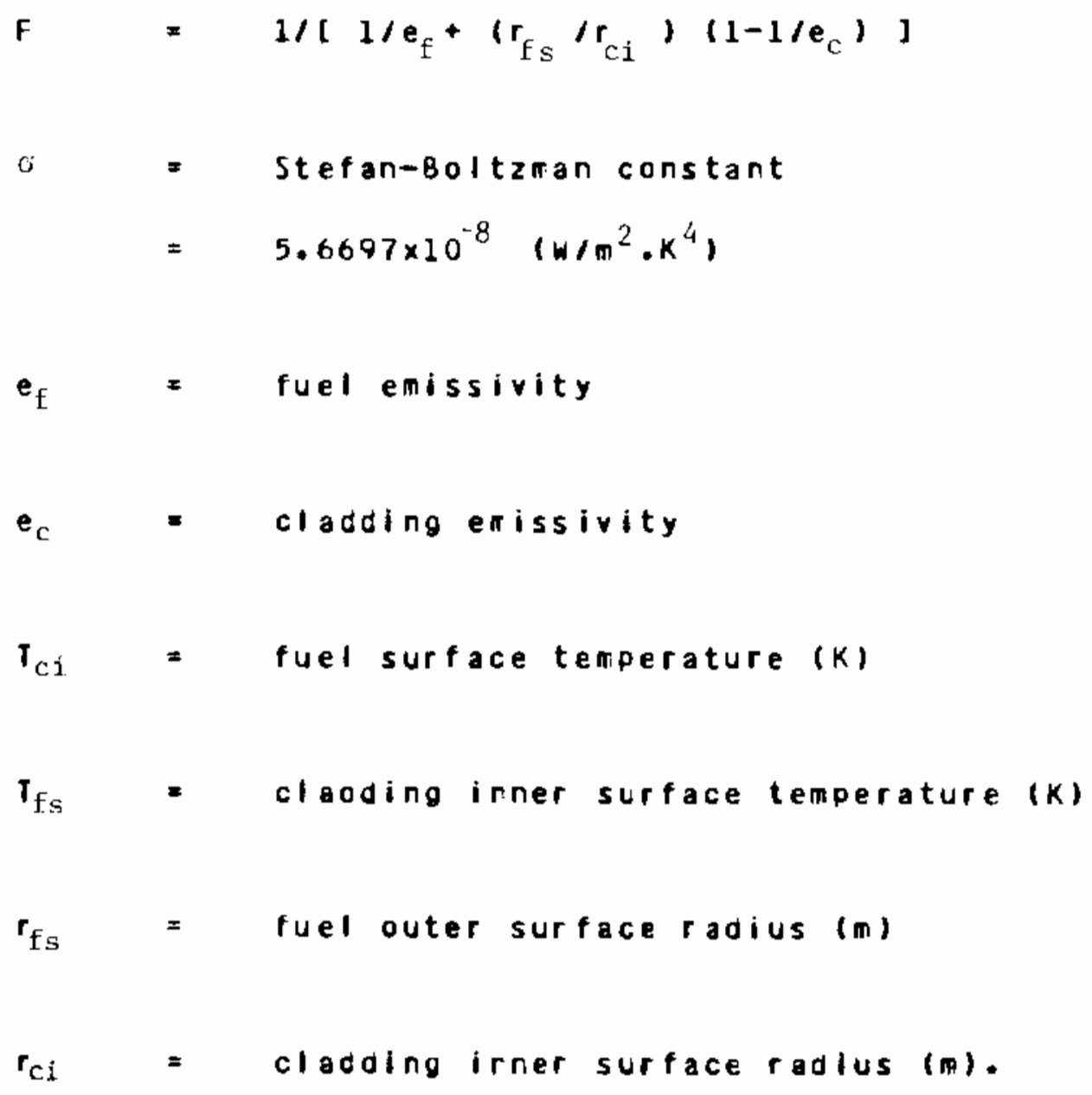

Tre conductance due to radiation, $h_{r}\left(w / w^{2}, k\right)$, is defined by 
$h_{r}\left(T_{f s}-T_{C i}\right)=$ S.H.F.

Combining Equations (11) and $(12)$ and dividing by $\left(1_{f_{s}}-T_{c i}\right.$ ) gives

$$
h_{z}=\sigma F\left[T_{f s}^{2}+T_{c i}^{2}\right]\left[T_{f s}+T_{c i}\right]
$$

Conduction Through The Interfacial Gas--ine form of the conductance due to conductive heat tranfer through the gas in the fuel-cladding gap gas, $h$ gas $\left(w / m^{2} \cdot k\right)$, is that universally applied to siall annular gaps:

$h_{\text {gas }}=\frac{k_{\text {gas }}}{d+\left(g_{1}+g_{2}\right)}=\frac{k_{\text {gas }}}{\Delta x}$

were

$$
\begin{aligned}
d \quad & \text { effective physical gap width }(\pi) \\
k_{\text {gas }} & =\text { sas thermal conductivity }(w / m \cdot k) \\
\left(g_{1}+g_{2}\right) & =\begin{array}{l}
\text { temperature jump distances at fuel and cladoino } \\
\text { surfaces, respectively }(\pi)
\end{array} \\
\Delta x \quad & =\text { total effective gap width }(m) .
\end{aligned}
$$


The various mechanical rodeling options separately contribute values of $\triangle x$. If either of the fRACAS mechanics options are chosen, $A x$ is given by 16

$$
\Delta \mathrm{x}=1.8\left[\mathrm{~d}_{\text {eff }}+\mathrm{g}_{1}+\mathrm{g}_{2}\right]-\mathrm{b}+\mathrm{d}
$$

where

$$
\begin{aligned}
& \text { d a value from fRACAS for open fuel-clacoing gap } \\
& \text { size (m) } \\
& d_{\text {eff }}=2 \exp (-0.00225 P)\left(R_{1}+R_{2}\right) \text { for closed fuel-cladoing } \\
& \text { gaps (m) } \\
& =2\left(R_{1}+R_{2}\right) \text { for open fuel-cladoing gaps }(m) \\
& \text { p = fuel-cladding interfacial fressure (kofer }{ }^{2} \text { ) } \\
& R_{1}+R_{2}=\text { cladding plus fuel surface roughness (R) } \\
& \text { b } \quad=1.397 \times 10^{-6}(m) \text {. }
\end{aligned}
$$

If the PELET option is chosen, fuel-cladding contact is almays assumed, and

$$
\Delta x=d_{\text {eff }}+1.8\left(g_{1}+g_{2}\right)
$$

whered is defined in the PELET/RAOIAL model. 
In elther case, the quantity $\left(\theta_{1}+g_{2}\right)$ is calculated from the GAPCDN-2 $2^{16}$ model which is

$$
\left(g_{1}+g_{2}\right)=A\left[\frac{k_{\text {gas }} \sqrt{T_{g a s}}}{P_{g a s}}\right]\left[\frac{1}{{\sum \beta_{i} f_{i} / \sqrt{M_{i}}}_{i}}\right]
$$

where

$$
\begin{aligned}
& A \quad=0.7816 \\
& k \text { gas }=\text { gas conouctivity }(m / m \cdot k) \\
& P_{\text {gas }} \quad \text { oas pressure }(\mathrm{Pa}) \\
& T_{\text {gas }}=\text { average gas temperature (K) } \\
& a_{i}=\text { accomodation coefficient of } i-t h \text { gas component } \\
& M_{i} x \text { gram-nolecular weight of i-th gas component iks. } \\
& \text { moles) } \\
& f_{i}=\text { mote fraction of i-th gas compcnent. }
\end{aligned}
$$

Conductance Through points of contact--The fRACAS and PELET mechanics models utilize expressions for h solid which are similar in form but differ in magnitude. If the FRACAS models are selecteo, $h_{\text {solid }}$ is dependent on both the fuel-cladcing interfacial pressure 
and the ricroscoplc roughnesses, $R$, as follows

$$
\begin{aligned}
& h_{\text {solid }}=A \mathrm{~km} \mathrm{P}_{\text {rel }} / \mathrm{RE}, \quad \mathrm{P}_{\text {rel }}>0.01 \\
& =A \mathrm{~km}(0.01) / \mathrm{RE}, 0.01>\mathrm{P}_{\mathrm{rel}}>0.0001 \\
& =\mathrm{A} \mathrm{km} \mathrm{P}_{\text {rel }}^{0.5} / \mathrm{RE}, \quad \mathrm{P}_{\mathrm{rel}}<0.0001
\end{aligned}
$$

where

$$
\begin{aligned}
& P_{\text {rel }}= \\
& \text { hatio of interfacial pressure to cladding Meyer }
\end{aligned}
$$

$k \pi=$ mean conductivity $(w / m \cdot k)$

$$
\begin{aligned}
& =2 K_{f} K_{c} /\left(K_{f}+K_{c}\right) \\
R & =\sqrt{R_{l}^{2}+R_{2}^{2}(m)} \\
K_{c} & =\text { cladding thermal conductivity }(w / m . K) \\
K_{f} & =\text { fuel thermal conductivity }(w / m \cdot K) \\
E \quad & \exp \left[-3.51-0.528 \ln \left(R_{1}\right)\right] .
\end{aligned}
$$

The above comes from a fit to Ross and stoute ${ }^{17}$ data plus that by Rapier ${ }^{18}$ using the Mikic-Todreas model. 19 
If the PELET option is chosen, h $h_{\text {solid }}$ is dependent only upon fuel-cladoing interfacial pressure, accordirg to the relation

$$
\mathrm{h}_{\text {solid }}=0.038+0.017 \mathrm{P}_{\text {int }}\left(w / \mathrm{cm}^{2} \cdot \mathrm{k}\right)
$$

where

$$
P_{\text {int }}=\text { fuel-cladoing interfacial pressure (MPa). }
$$

This equation is an estiratec fit to recent out-of-pile cata by J.A. Garnier. 20

2.3.5 Pellet Heat conouction. The pellet temperature distribution is calculated using the Method of weighted Residuals proposed by Finlayson. 21 The methoo is described below.

2.3.5.1. General Procedures--The rodel used to solve the steady state radial heat transfer in the fuel is based on the assumption that the fuel is a homogeneous, continuous right circular cylinder with a constraint surface temperature about its perimeter. The method used has been extended to hancle a restructured zone. Heat conouction in the radial direction in the fuel is described by

$$
\frac{d^{2} T(r)}{d r^{2}}+\frac{1}{r} \frac{d T}{d r}+\frac{1}{K(T)}\left(\frac{d K}{d T}\right)\left(\frac{d T}{d r}\right)^{2}+\frac{Q(r)}{K}=0
$$


where

$$
\begin{aligned}
& I(r)=\text { tre fuel temperature }(K) \\
& Q(r)=\text { the volumetric heat generation rate (wfm }{ }^{3} \\
& =\quad \text { the conductivity of the fuel }(W / m \cdot K) .
\end{aligned}
$$

The equation is nonlinear because of the temperature dependence of the fuel conductivity. The boundary conditions are

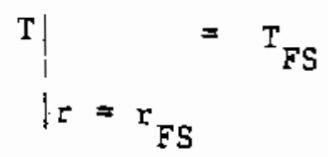

and

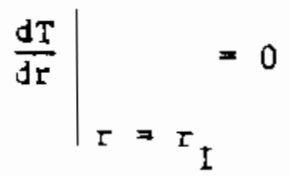

where

$\begin{array}{ll}r_{I} & \text { fuel inner radius }(\mathrm{m}) \\ r_{F S} & =\text { fuel outer radius }(\mathrm{m}) \\ T_{F S} & =\text { fuel surface temperature }(K) .\end{array}$


There are several methods which can be used to solve Equation (20). The method used in FRAPCGN-2 is a collocation technique' using the method of weighted residuals, and has the following advantages: (a) the solution time on a computer is minimal, and (b) coefficients are produced which permit calculation of correct temperatures at any radial position in the fuel without resolving the entire problem. The specific steps used are a slight variation of the method proposec by B. A. Finlayson, and proceed as follows:

I. A trial solution of the form $T=\sum_{j=1}^{N+2} d_{j} r^{j-1}$ is assumed, where the $d_{j}$ are unknown coefficients.

2. To find estimates of the $d_{j}$, Equation (20) is forced to be satisfied at $N$ coflocation points within the fuel or annular sutreoion. This requirement plus the boundary conditions result in $N+2$ simultaneous equations for the $\mathrm{N}+2 \mathrm{~d}_{j}$ coefficients. Fourth-order (4 point) collocation has been found to yield temperatures accurate to within 1 k compared with exact solutions. Thus, six coefficients are gerierated.

3. In order to solve the six simultaneous equations, the terms involving conductivity are considered knonn by evaluating the conductivity using temperatures from the previous iteration. The temperatures for the very first iteration are calculated using a constant typical value for the conductivity.

4. The procedure is repeated until convergence of the 
2.3.5.2 Formulation of Equations for $o_{j}$-lo avoio reformulating the equations for each gap conductance lteration, it is convenient to perform the calculations in the normalized cooroinate, $y=r / r_{F S}$. With this variatle change, Equation ( 20 ) becomes

$\frac{d^{2} T(y)}{d y^{2}}+\frac{1}{y} \frac{d T}{d y}+\frac{1}{k(T)} \frac{d K}{d T}\left(\frac{d T}{d y}\right)^{2}+\frac{Q(y) r^{2} F S}{K}=0$

with boundary conditions

$$
\begin{aligned}
& \frac{d T}{d y}: y=y_{I} \\
& \quad=0 \\
& \left.T\right|_{y=1}=T_{\text {FS }} .
\end{aligned}
$$

Using the collocation technique, six simultaneous equations are formulated as

$$
\left.\frac{d T_{n}}{d y}\right|_{y=y_{I}}=0
$$

$\left.\frac{d^{2} T_{n}}{d y^{2}}\right|_{y=y_{i}}+\left.\frac{1}{y_{i}} \frac{d T_{n}}{d y}\right|_{y=y_{i}}=\frac{1}{K_{n-1}}\left[r^{2} Q_{i}+\left(\frac{d k_{n}-1}{d T_{i}}\right)\left(\left.\frac{d T_{n}-1}{d y}\right|_{y=y_{i}} ^{2}\right)_{i=2,3,4 \text { and } 5}\right]$ 
$\left.\mathrm{T}_{\mathrm{n}}\right|_{\mathrm{y}=1}=\mathrm{T}_{\mathrm{FS}} \quad \mathrm{i}=6$

where sutscripts $n$ and $n-1$ refer to the current and previous iteration, respectively, anc

$Q_{i}=Q\left(y_{i}\right)$,

$x_{n-1}=x\left(T_{n-1}\right)$

$\frac{d x_{n-1}}{d T_{i}}=\left.\frac{d X}{d T}\right|_{T=T_{n-1}\left(y_{i}\right)}$

The equations for $i=2$ through s need to be modified by elminating thé tern

$\left(\left.\frac{d T_{a-1}}{d y}\right|_{y=y_{i}}\right)^{2}$

Ire most accurate way to do this is to use the relationship

$-\left.K \frac{d T}{d r}\right|_{r=r_{i}}=$ surface heat flux at $r_{i}=2 \pi \int_{r_{I}}^{r} Q(r) r d r / \pi 2 r$. 
$Q(r)$ is expressea as $Q(r)=N\left(Z+Y Y r^{2}+w r^{4}\right.$, where $N$ is a norralization constant which transforms $O(r)$ into physical units. Thus, Equation (33) tecomes

$$
-K \frac{d T}{d r}=\frac{N}{r}\left[\frac{Z}{2}\left(r^{2}-r_{I}^{2}\right)+\frac{Y Y}{4}\left(r^{4}-r_{I}^{4}\right)+\frac{H}{6}\left(r^{6}-r_{I}^{6}\right)\right] .
$$

Iransforming to normalized coordinates ano solving for $\frac{d T}{d y} \bigcup_{y=y_{i}}$
Equation (34) Decomes

$$
\begin{aligned}
& \left.\frac{d T}{d y}\right|_{y=y_{i}}=-\left(\frac{N \tau_{S S}^{2}}{y_{i}^{K}}\right)\left[\frac{z}{2}\left(y_{i}^{2}-y_{I}^{2}\right)+\frac{Y Y}{4}\left(y_{i}^{4}-y_{I}^{4}\right)+\frac{W}{6}\left(y_{i}^{6}-y_{I}^{6}\right)\right] \\
& =\left[\frac{N r_{E S}^{2}}{y_{i} K}\right] C_{i} \equiv I_{i} .
\end{aligned}
$$

Thus, the collocation equations $j=2$ through 5 can be mritten as

$$
T_{n}\left(y_{i}\right)=\sum_{j=1}^{6} d_{j} y_{i}^{j-1}
$$

with I defined in Equation (36). Substituting the following expression into Equation (37),

$$
\left.\frac{d^{2} T}{d y^{2}}\right|_{y=y_{i}}+\left.\frac{1}{y_{i}} \frac{d T}{d y}\right|_{y=y_{i}}=\frac{-1}{K_{n-1}}\left[r_{F S}{ }^{2} Q_{i}+\left(\frac{d K_{n-1}}{d T_{i}}\right) I_{i}{ }^{2}\right]
$$


the following six equations are obtainea:

$$
\sum_{j=1}^{6} d_{j}\left[(j-1) y_{i}^{j-2}\right]=0 \quad i=1
$$

$\sum_{j=1}^{6} d_{j}\left[\left(j^{2}-2 j+1\right) y_{i}{ }^{-3}\right]=\frac{-1}{k_{n-1}}\left[r_{F S}{ }^{2} Q_{i}+\left(\frac{d k_{n-1}}{d T_{i}}\right) I_{i}{ }^{2}\right] i=2,3,4$, and 5

$$
\sum_{j=1}^{6} d_{j}=T_{F S} \quad 1=6
$$

In watrix form, the above set can be written as

$$
[A]\left(\begin{array}{l}
d_{1} \\
d_{2} \\
d_{3} \\
d_{4} \\
d_{5} \\
d_{6}
\end{array}\right)=\left(\begin{array}{l}
0.0 \\
B_{2} \\
B_{3} \\
B_{4} \\
B_{5} \\
T_{F S}
\end{array}\right)
$$


where

$$
\left.\begin{array}{rlrl}
A_{i j} & =(j-1) y_{I}(j-2), & i & =1 \\
& =\left({ }^{2}-2 j+1\right) y_{i}^{j-3,}, & i & =2,3,4,5 \\
& =1.0 & i & =6
\end{array}\right\} j=1,2,3,4,5,6
$$

anc

$B_{i}=\frac{-1}{K_{n-1}}\left[r_{F S}{ }^{2} Q_{i}+\left(\frac{d K_{n-1}}{d T_{i}}\right) I_{i}^{2}\right], i=2,3,4,5$.

Notice that in succeeding iterations, the matrix a need not be recalculated, but only the $\theta_{i}$.

This procedure can be appliec to annular subregions of the fuel, which are then couplea by the condition of tenperature ano heat flow continuity between regions. The motive for doing so comes from the possibility for deviation in the fuel thernal concuctivity function above ano below a transition temperature isee section $2 \cdot 3 \cdot 7)$.

2.3.6 Plenum Gas Temperature. The plenun gas temperature is calculated based on energy transfer between the top of the pellet stack and the plenum gas, between the coolant channel and the plenum gas, and between the spring and the plenum gas. A discussion of these contributions follows. 
ivatural convection from the top of the tuel stack is calculated based on heat transfer coefficients from Mcadams 22 for laminar or turuulent natural convection from flat plates.

The heat transfer coefficient is calculated from

$h_{p}=\frac{k N u}{D}$

where

$$
\begin{aligned}
& h_{P}=\text { the heat transfer coefficient from the top of the } \\
& \text { pellet stack to the olenum gas }\left(w / m^{2}, K\right) \\
& \text { Nu } \quad=\text { Nusselt Number } \\
& 0 \text { = inside diameter of the cladding of the top node (m) } \\
& k=\text { conductivity of the plenum gas }(\mathrm{w} / \mathrm{m}, \mathrm{k}) \text {. }
\end{aligned}
$$

The Nusselt Number is calculated using

$\mathrm{Nu}=\mathrm{C}\left(\mathrm{GrPr}_{\mathrm{r}}\right)^{\mathrm{m}}$

where

$$
\text { Gr } \quad=\text { the Grashof Number }
$$


Pr = the Pranat I Number

and for

GrPr $\leq 2.0 \times 10^{7}, \mathrm{C}=0.54$ and $\mathrm{m}=0.25$,

or

GrPr > $2.0 \times 10^{7} \mathrm{C}=0.14$ and $\mathrm{m}=0.33$.

The overall effective conouctivity from the coolant to the plenum is defined as the inverse of the sum of the individual heat flon resistances. The three resistances are (a) the resistance across the inside surface film, (b) the resistance across the cladding, and (c) the resistance across the outside surface film. The overall conductivity is therefore found as

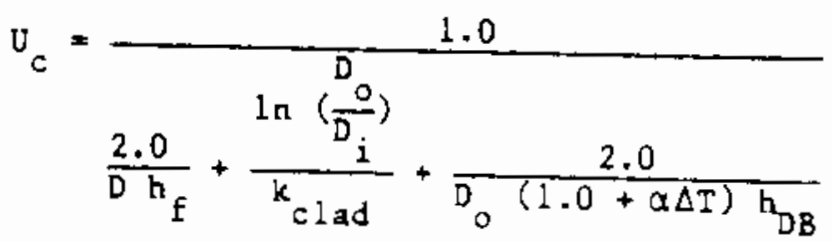

where
$U_{c}=$ overall effective conductivity from the coolant to the plenum gas $(m / m \cdot k)$
D $\quad$ hot-state inside cladding diameter (m)
$h_{f}=$ cladoing inside surface film coefficient $\left(W / \pi^{2}, K\right)$ 


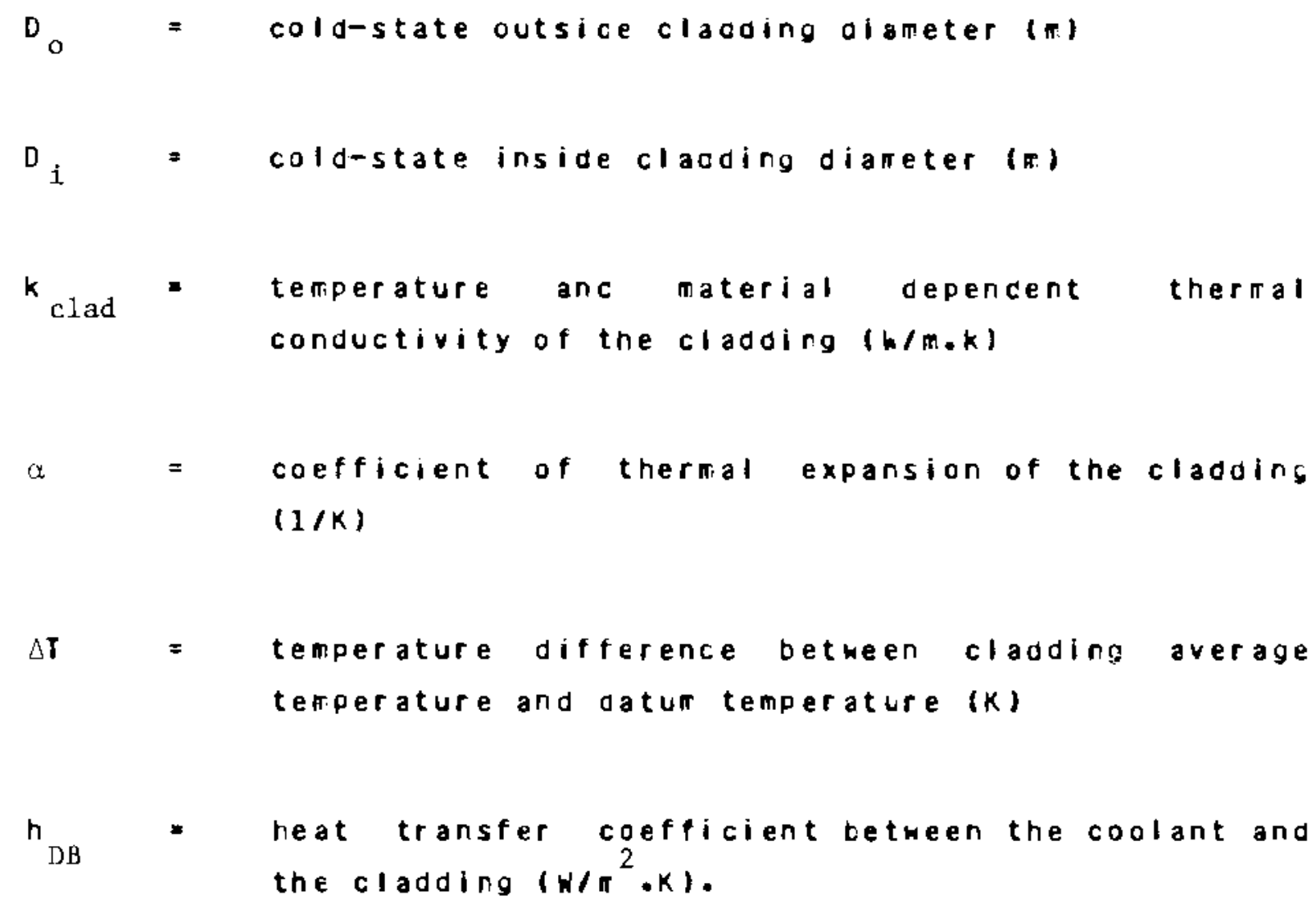

Gamia heating in the hold down spring is calculated assuring a volumetric heating rate of $3.76 \mathrm{~m} / \mathrm{m}^{3}$ for every $/ \mathrm{m}^{2}$ of rod average heat flux. The expression is

$Q_{s p}=3.76 \dot{q} " v_{s}$

where
$Q_{\mathrm{sp}}=$ energy generated in the spring due to gamma teating (w)
$\dot{q}^{\prime \prime} \quad=\quad$ average heat $f\left(u x\right.$ of the $\operatorname{rod}\left(w / m^{2}\right)$ 
$v_{s}=$ volume of the spring $\left(\pi^{3}\right)$

The plenum temperature is approximated from

$T_{\text {plen }}=\frac{Q_{s p}+U_{c} \frac{v_{p}}{D^{2}} T_{B L K}+T_{p a} h_{p} \pi D^{2} / 4}{U_{c} \frac{V_{p}}{D^{2}}+\frac{h_{p} \pi D^{2}}{4}}$

where

$$
\begin{aligned}
& T_{\text {plen }}=\text { plenum temperature }(K) \\
& v_{p} \quad \text { volume of the plenum }\left(\pi^{3}\right) \\
& T_{B L K}=\text { bulk coolant temperature at the top axial noce }(K) \\
& T_{p a} \quad=\text { temperature associateo with the insulator or top } \\
& \text { pellet }(K) \text {. }
\end{aligned}
$$

2.3.7 Effective fuel Conductivity. The thermal conductivity of cracked fuel is not the same as trat of uncrackeofuel. In order to compute the effective thermal conductivity of cracked fuet, a conductivity factor, $R, i s$ applied to the uncrackec fuel conductivity. The conductivity factor is

$k_{\text {eff }}=R k_{\text {lab }}$ 
where

$$
\begin{aligned}
& k_{\text {eff }}=\text { effective fuel thermal concictivity (w/m.k) } \\
& k_{\text {lab }}=\text { uncracked fuel thermal conouctivity as a function } \\
& \text { of temperature }(\mathrm{m} / \mathrm{m} \cdot \mathrm{k}) .
\end{aligned}
$$

2.3.7.1 The Cracked Conouctivity factor Used with FRACAS-DIRe cracked conductivity factor used hith fRACAS is an emcirical ccrielation which accounts for the presence of cracks in the fuel. This correlation was developed trom fuel centerline and offcenter theriocouple data taken as part of the jap conouctance tests series $^{23}$ performed in the pomer Burst facility at the Idaho National Engineering Laboratory. Ine equation for $R$ is

$$
R=1.0-C c_{r e l}\left[1.0-\frac{k^{g}}{k_{1 a b}}\right]
$$

where

$$
\begin{aligned}
& \text { C }=0.30(1 / \mathrm{m}) \text { for FRACAS-I } \\
& =0.48(1 / \mathrm{m}) \text { for FRACAS-II } \\
& C_{\text {rel }}=\frac{3 \delta\left(\delta_{s}-\delta_{\mathrm{T}}\right)}{\mathrm{r}_{\mathrm{p}} \mathrm{V}\left(0.8 \times 10^{-4}\right)} \text { (n) for HRACAS- I } \\
& =\frac{V}{4 r_{p}\left(0.8 \times 10^{-4}\right)}(n) \text { for FRACAS-II }
\end{aligned}
$$




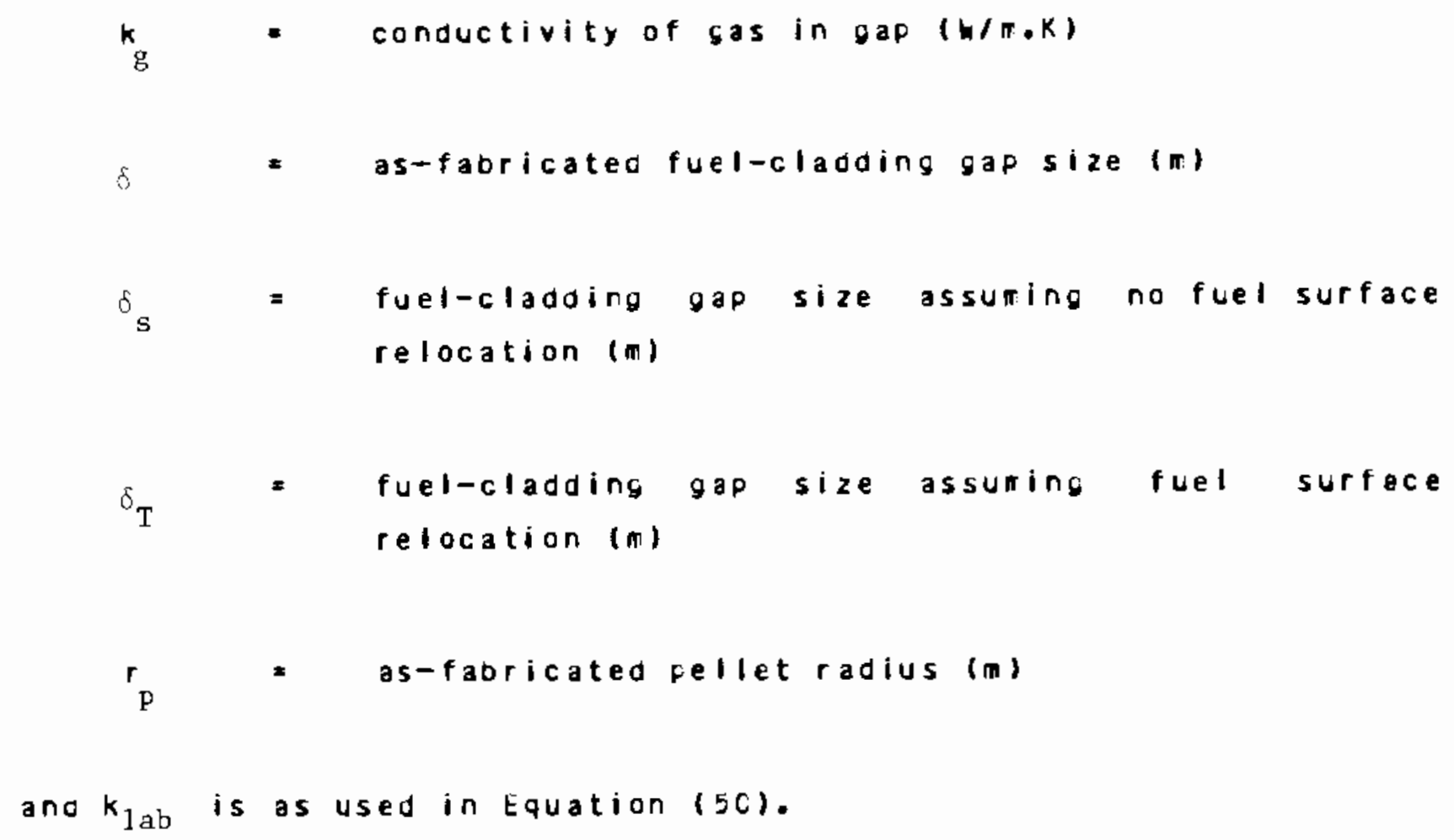

The term $\left(1-k_{g} / k_{l a b}\right)$ accounts for the fact that the gas in the cracks in the fuel has a lower conductivity than the fuel, and thus degrades the effective conductivity of the fuel.

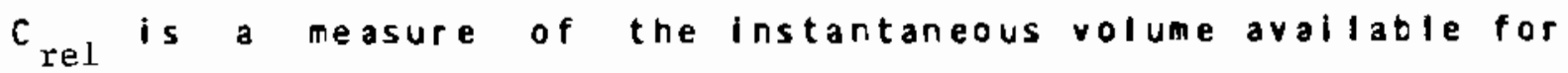
cracking. This term oecreases as the fuet-cladoing gap size decreases. Recognizing that crackeo fuel can never fully reconsolidate, this term is never allowed to be less than 0.25 . C was chosen to best fit the experimental data base for roos containing He, $x e$, and ar.
\end{abstract}

Crack healing is assumed when the local fuet temperature reaches cr exceeds some transition temperature. The transition temperature has been chosen to be nine-tenths of the user-specifieo fuel sintering temperature. Crack healing is enforced as 
$R=1.0$ when $T_{I} \geq T_{\text {trans }}$

where

$$
\begin{aligned}
& T_{r}=\text { local fuel temperature }(K) \\
& T_{\text {trans }}=\text { fuel transition temperature }(K) .
\end{aligned}
$$

Assumptions made are that (a) cracks instantaneously heal when fued regions are above the transition temperature, (b) once a region has healed no recracking of that region accurs and (c) the relative $g$ as and fuel conductivities are latoratory values.

2.3.7.2 The Effective Fuel Conductivity Used with PELET/ RADIAL-- Ine effective value of tre fuel thermal conductivity is degraded by fuel cracking. This follows directly from the otservation that the cracks in fuel pellets observed in postirradiation examinations have nonradial components. As notedin Section 2.2.2, certain assigned values for crack length and fuel and gap roughness are given to various rods from mich in-reactor powert temperature measurements are avaifable. The assignment of these values permits the calculation of fuel-clading gap size, gap conductance, and hycrostatic fuel stress. From the gap conductance, fuel surface temperature can be estimated. The estimateo surface temperature, $I_{s}$, ano measured fuel centerline temperature, $I_{c}$, can be used to calculate the effective fuel conductivity as 
CFAC $\int_{T_{S}}^{\mathrm{T}} \mathrm{K}_{\mathrm{M}} \mathrm{dT}=\frac{\mathrm{Pf}}{4 \pi}$

where

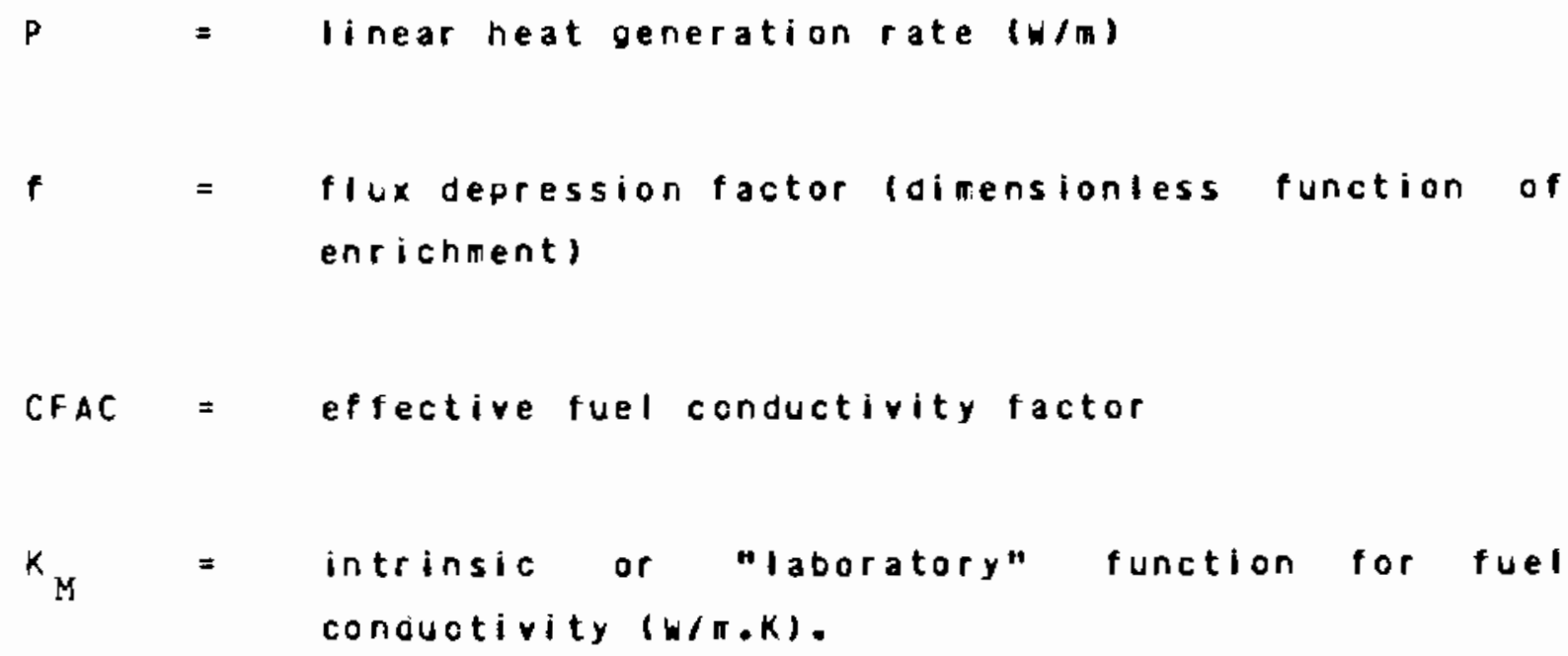

Generally, CFAC is found to be less than 1.0. A plot of CFAC values versus the corresponding fuel hydrostatio stress is shom for various Halden roos in figure 5.

Note in figure 5 that the CFAC values for all of the helium filled rods teno to overlap (except at exceedingly lon stress) anc tend to be linear. Simllarly the data from the mixea-gas rod also tend to be linear. This is the basis for the correlation that is applied in FRAPCOA-2 when the PELET/RADIAL option is used. The dependence of the conductivity for given rod is nearly linear, but the slope and intercept of the lines are functions of the gas 


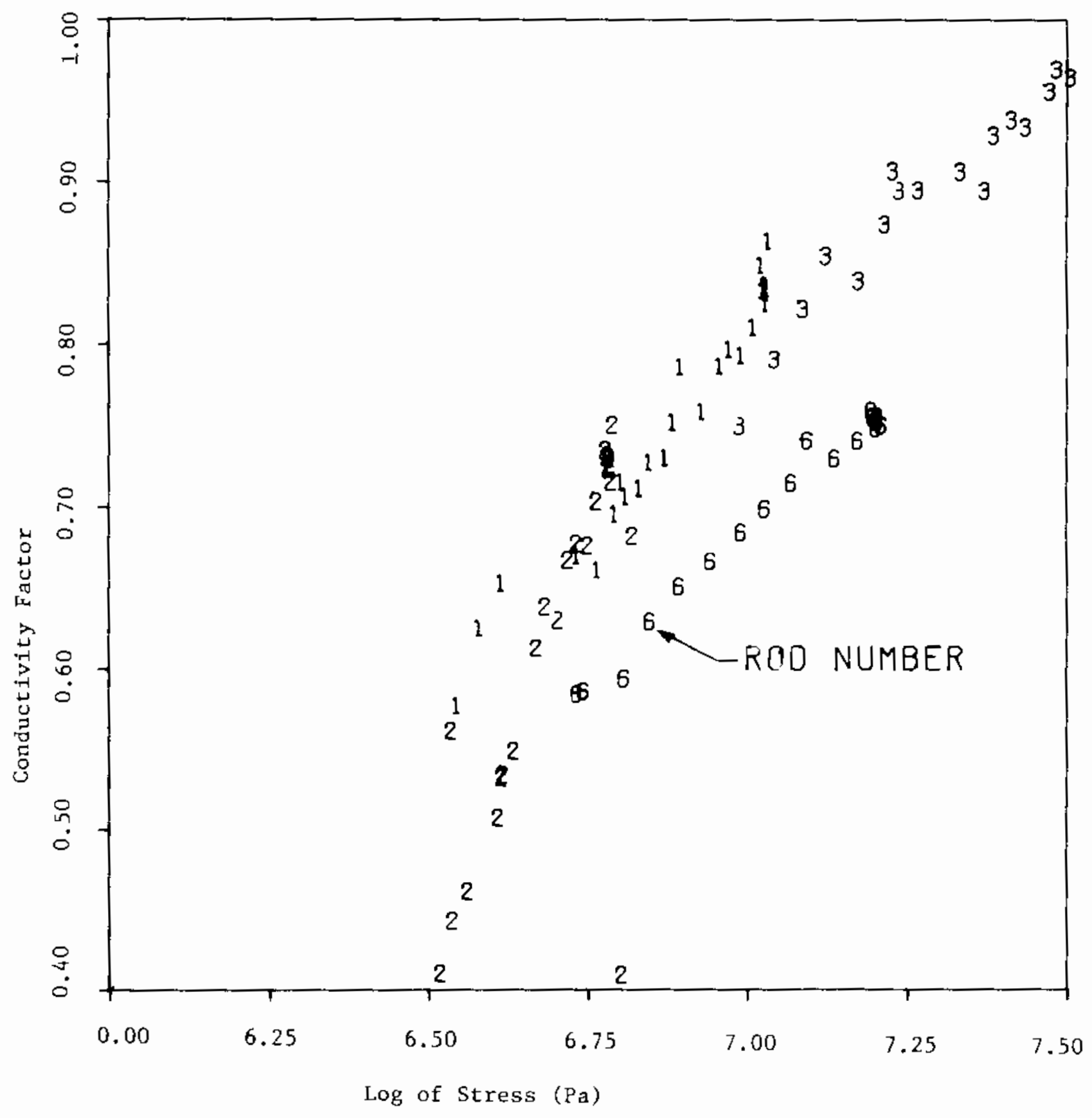

Figure 5. Thermal conductivity factor versus hydrostatic stress (data points identified by rod number). 
conductivity relative to that for pure helium.

Figure $t$ shows the correlation for CFAC as it is applied in RACIAL. Note that the low-cutoff for the conouctivity multiplier (CFAC) has teen arbitrarily chosen as 0.3 . Note also that even at zero power, convergeo values for the hydrostatic stress are ir. excess of $1.4 \times 10$ Pa $(200$ psial which is why the correlation is based on the intercept at log(stress) = 6 plus the slope estimatec from data. The correlations for intercept and slope of CFAC versus log(stress) as a function of gas conductivity ratio (the gas conductivity ratio is defined as the ratio of current gas conguctivity to pure helium gas conductivity) are shom in figures? and 8 , respectively. Note that corbinations of slope, intercept ano log(stress) resulting in CFAC values less than 0.3 are aiscountec since 0.3 is the lon-value cutoft for CFAC.

2.3.8 Stored Energy. The stored energy in the fuel rod is calculated by suming the erergy of each pellet ring calculated at the ring temperature. The expression for stored energy is

$$
E_{S}=\frac{\sum_{i=1}^{I} m_{i} \int_{298 k}^{T_{i}} c_{p}(t) d T}{m}
$$

were

$$
\begin{aligned}
& E_{S} \quad=\text { stored energy }(J / k g) \\
& m_{i}=\text { mass of ring segrent } i(k g)
\end{aligned}
$$


or

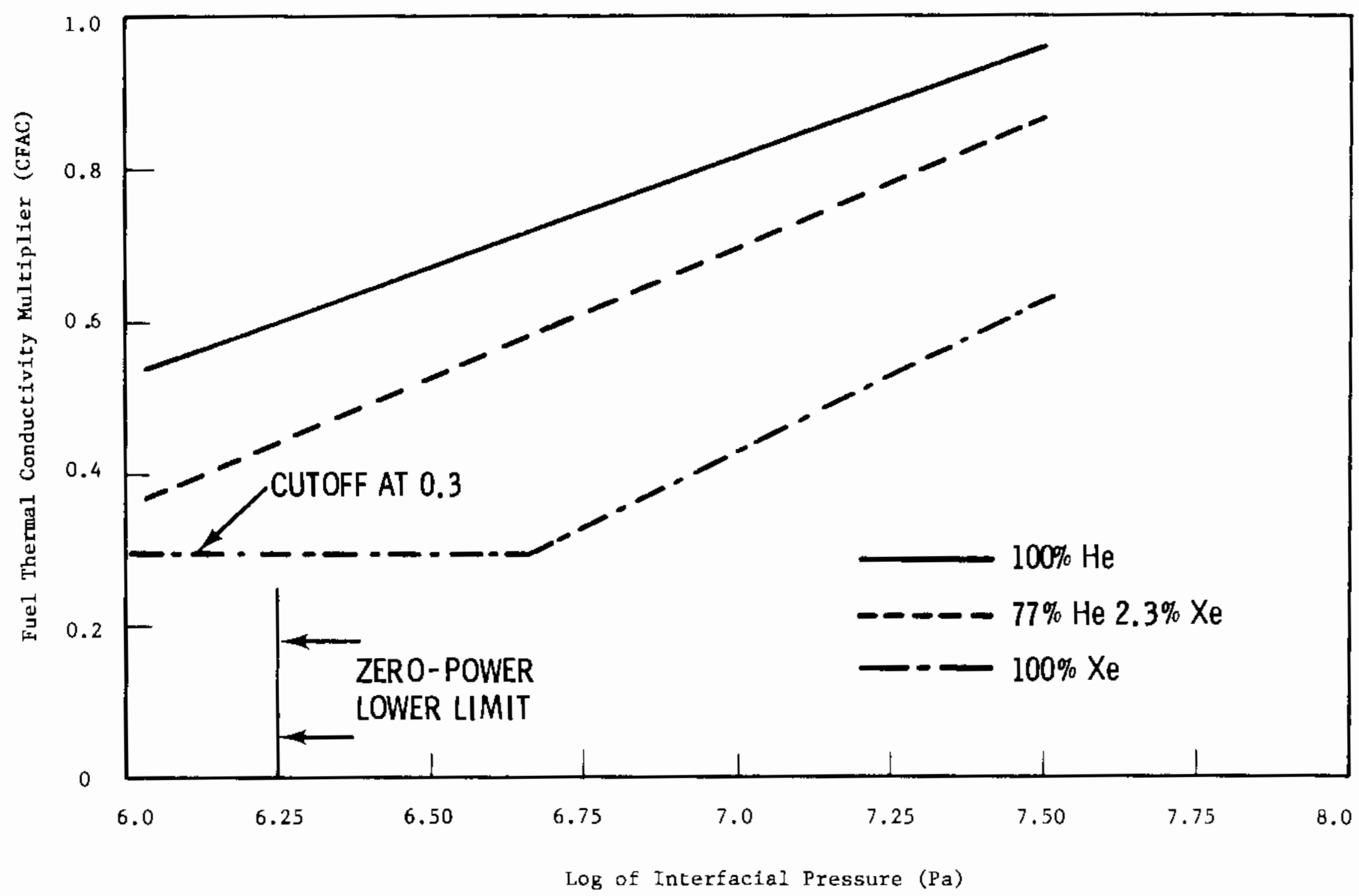

Figure 6. Fuel conductivity multiplier (CFAC) as a function of interfacial pressure and gas composition. 


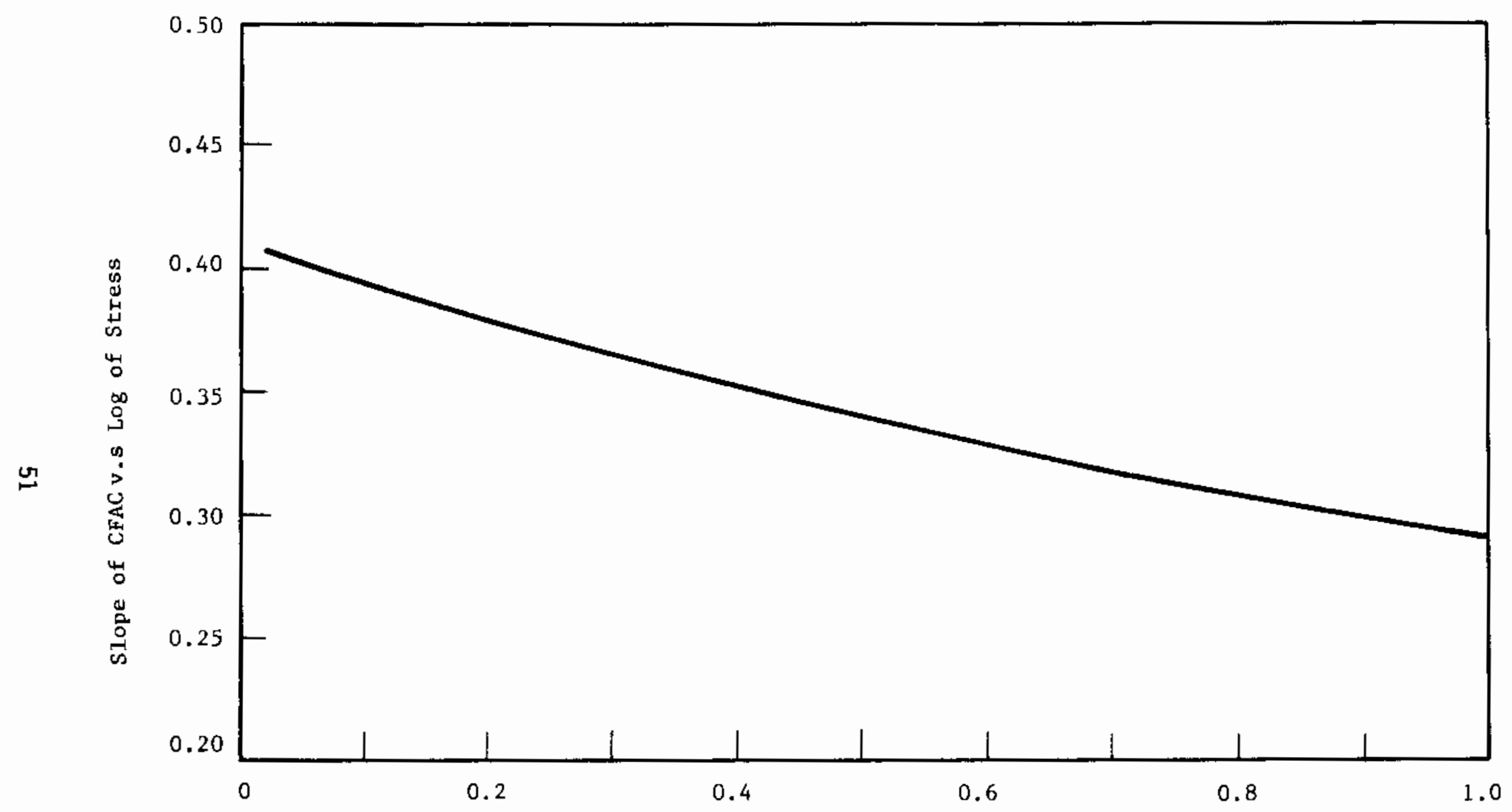

Ratio of Gas Conductivity to That for Pure Helium

Figure 7. Slope of CFAC versus log (pressure) curve as a function of fill gas conductivity. 


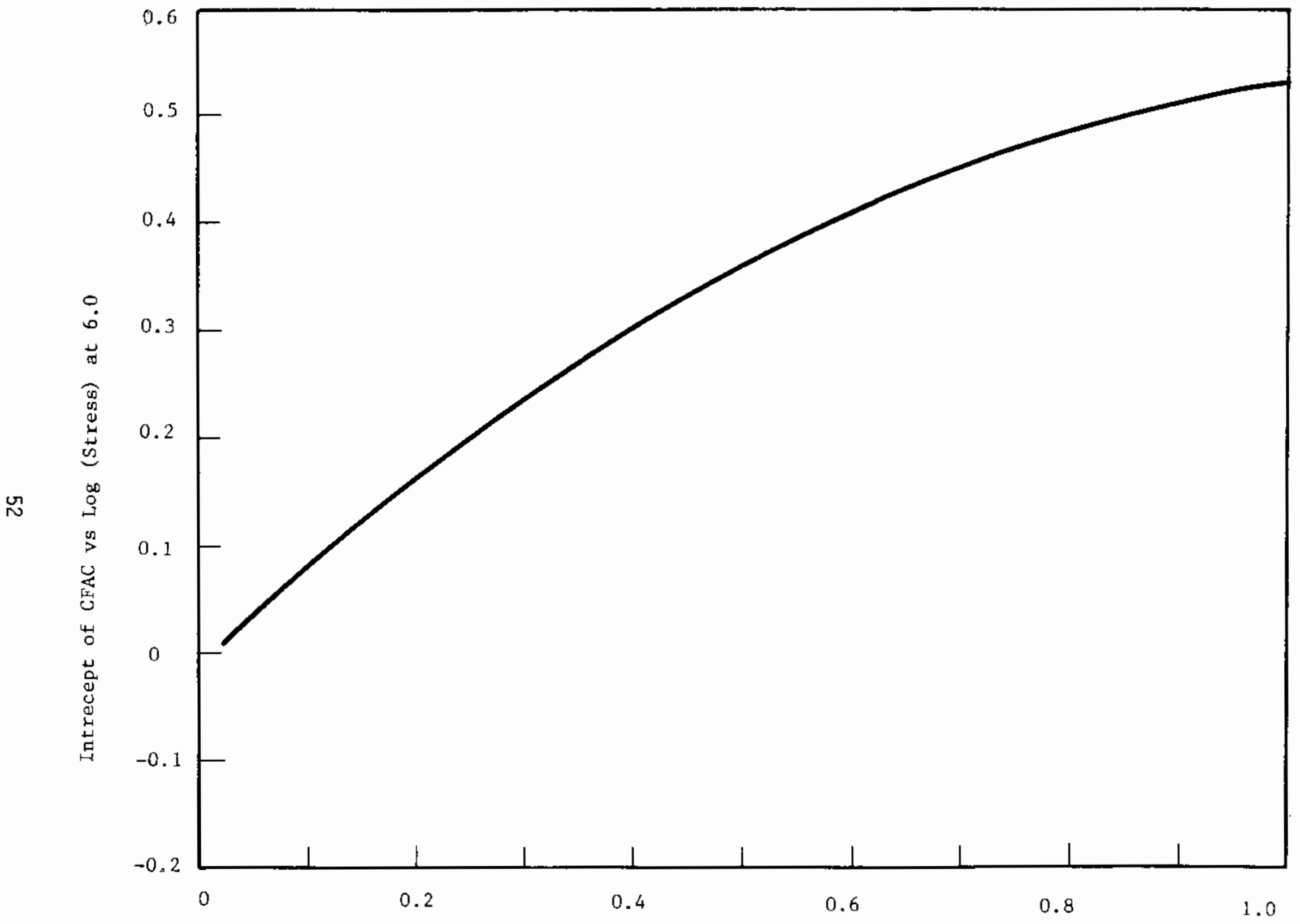

Figure 8. Intercept of CFAC versus $\log$ (pressure) curve (at 6.0) as a function of fill gas conductivity. 


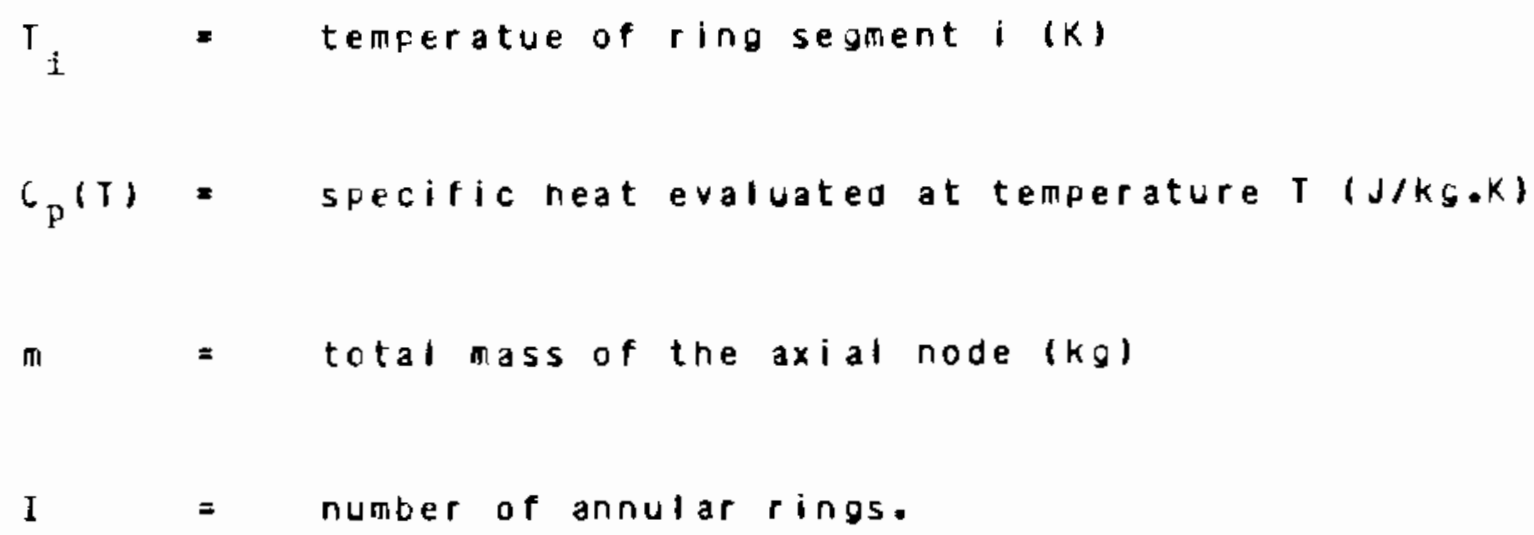

An accurate calculation of fuel and cladding deformation is necessary in any fuel rod response analysis because the heat transfer coefficient across the tuel-cladding gap is a function of both the effective fuel-cladding gap size and the fuel-clading interfacial pressure. In addition, an accurate calculation of stresses in the cladding is needed so that an accurate calculation of the onset of cladaing failure land subsequent release of fission productsl can be made.

FRAPCON-2 has four deformation modeling options: FRACAS-I, FRACAS-II, PELET/RADIAL and AXISYM. The models are described in the following sections.

2.4.1 The FRACAS models. Two FRACAS models are arailable for the calculation of the small displacement deformation of the fuel and cladoing. The more simplified model, FRACAS-I, neglects the stressinduced deformation of the fuel, and is called the riglo peliet 
model. The seccnd option, fkacas-II, includes stress-induced fuel deformation, and is calted the deformable pellet model.

In analyzing the deformation of fuel rods, two physical situations are envisioned. Tne first situation occurs when the fuel and clajding are not in contact. Here the problem of a cylindrical shetl (the clasoing) with specified internal and external pressures and a specified cladoing temperature distribution must be solved. Ihis situation is calleo the "open gap" regime.

The second situation envisioned is when the fuel considerably hotter thar the cladoingl has expanded so as to be in contact with tne cladoing. Further heating of the fuel results in "driving" the cladoing outwardy. Inis situation is called the "closed gap" regime. Alternatively, this closed gap regime can occur due to the creep of the cladoirg onto the fuel due to elevated clacding temperatures anj a high coolant pressure.

The prececing two regimes of fuel rod deformation are characterized by siall cladding strains and by the clacoing retaining its essentially cylindrical shape.

The deformation analysis in FkAPCON-2 consists of a 5 mialldeformation analysis ano when Using FRACAS-I, a cladoing failure analysis. A small deformation analysis of the stresses, strains and displacements in the tuel anc cladding is performed first for the entire fuel rod. This analysis is based on the assumption that the cladoing retains its cylindrical shape during deformation, and includes the effects of:

1. Fuel thermal expansion, creep, swelling, densification and 
relocation

2. Cladding thermal expansion, creep and plasticity

3. Fission sas and external coolant pressures.

As part of the small displacement andysis, the applicable local deformation regime (open gap, or closedgap) is determirea. Finally, an analysis is performed to oetermine if cladding failure has occured.

In section 2.4.1.1, the general theory of plastic analys is is outined and the method of solution used in tine fRACAS models is presented. Ihis method of solution is used in toth the rigid pellet and deformatle pellet models. In Sections 2.4.1.2, and 2.4.1.3, the equations for the rigid pellet model ano deformable pellet model, respectively, are oescribea.

2.4.1.1 General Theory ana Method of Solution--The general cheory of ulastic analysis and the method of solution are used in both the rigid pellet and deformable pellet models.

General consicerations in Elastic-plastic Analysis--Problems involving elastic-plastic deformation and multiaxial stress states involve a number of aspects that do not require consideration in a uniaxial protlen. In the following discussion, an at tempt is made to uriefly outlire the structure of incremental plasticity, ana to outline tre Method of Successive Substitutions ${ }^{22}$ lalso called the Methoo of Successive Elastic Solutionsl, which has been used successfully in treating muttiaxial elastic-plastic problems. The 
method can be used for any problem for which a solution based on elasticity can be obtainea. Tris method is used in both the rigio pellet and deformable pellet models.

In a problem involving only uniaxial stress, $\sigma_{1}$, the strain, $\varepsilon_{1}$, is related to the stress by an experimentally determined stress-strain curve as shown in figure 9, and Hooke's law is taken as

$$
\varepsilon_{1}=\frac{\sigma_{1}}{E}+\varepsilon_{1}^{p}+\int \alpha d T
$$

where $E_{1}^{P}$ is the plastic strain and $E$ is the modulus of elasticity. The onset of yieldino occurs at the yield stress, which can be determined directiy from fisure 9. Given a load (stress) history, the resulting deformation can be determined in a simple manner. The increase of yield stress with work-nardening is easily computed directly from ficure 9.

In a probler. involving multiaxial states of stress, however, the situation is not as clear. In such a problem, a method of refating the onset of plastic deformation to the results of a uniaxial test is required, and further, when plastic deformation occurs, some means is needed for determining how much plastic deformation has occurred and how that deformation is distributed among the individual components of strain. These tmo complications are taken into account by use of the so-called "yield function" and "flow rule", respectively. 


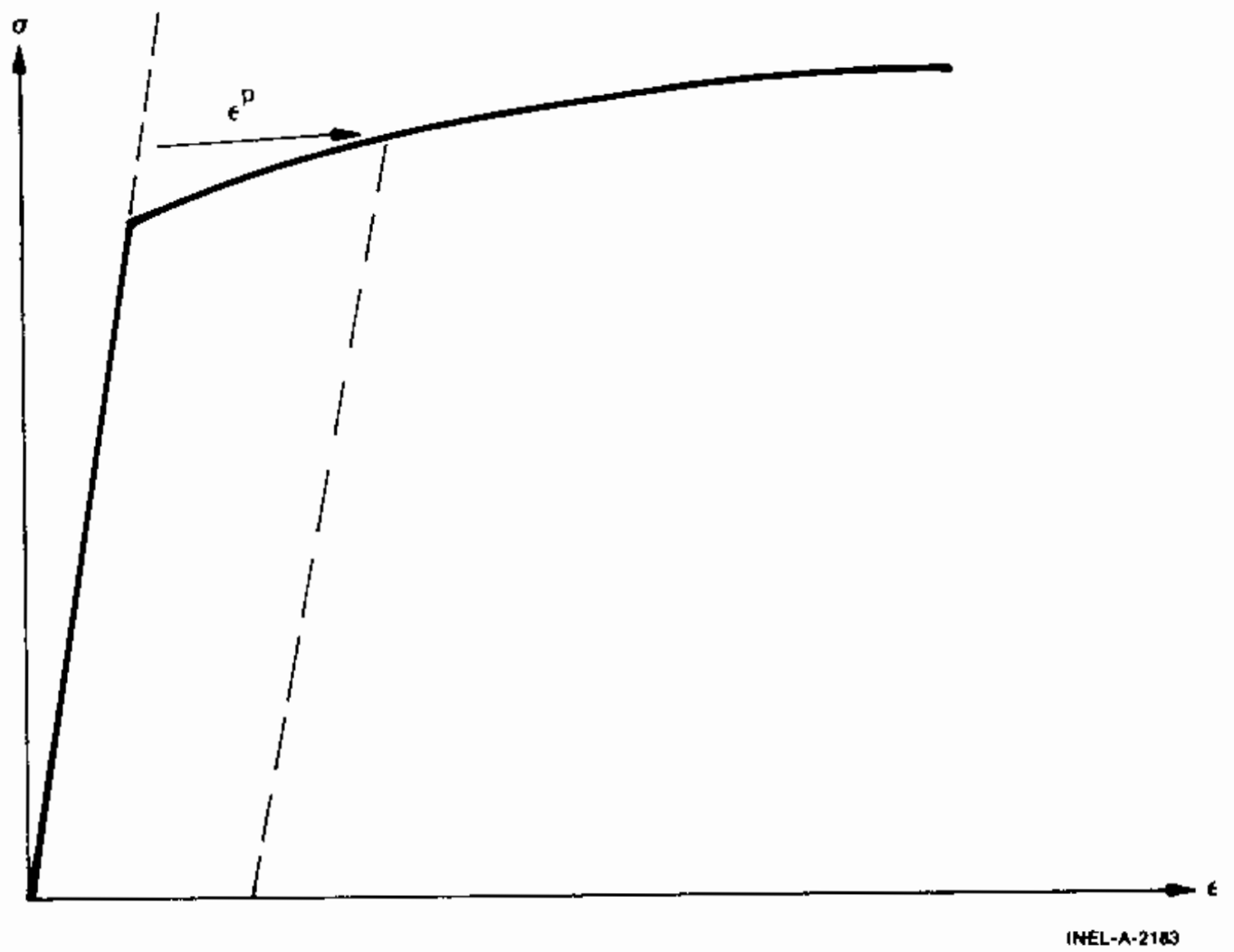

Figure 9. Typical isothermal stress-strain curve.

A considerable wealth of experimental evidence exists on the onset of yieloing in a mulitaxial stress state. The bulk of this evidence supports the von Mises yield criterion, which asserts that yieloing occurs when the stress state is such that

$$
0.5\left[\left(\sigma_{1}-\sigma_{2}\right)^{2}+\left(\sigma_{2}-\sigma_{3}\right)^{2}+\left(\sigma_{3}-\sigma_{1}\right)^{2}\right]=\sigma_{y}^{2}
$$

where the $\sigma_{1}$ values are the principle stresses, and $\sigma_{y}$ is the yletd stress as determined in a uniaxial stress-strain test. The square root of the left side of this equation is referred to as the 
"effective stress", "e, and this effective stress is one commonly used type of yield function.

10 determine how the yiela stress changes with cermanent deformation, the yield stress is hypothesized to be a function of the equivalent flastic strain, $\varepsilon^{2}$. An increment of equivalent plastic strain is determined at each load step, and $\varepsilon^{p}$ is defined as the sum of all increments incurred:

$\varepsilon^{P} \triangleq \quad \sum d \varepsilon^{P}$

bach ircrement of effective plastic strain is related to the inaividual plastic strain components by

$$
\begin{gathered}
\mathrm{d} \varepsilon^{\mathrm{P}}=\frac{\sqrt{2}}{3}\left[\left(\mathrm{~d} \varepsilon_{1}^{\mathrm{P}}-\mathrm{d} \varepsilon_{2}^{\mathrm{P}}\right)^{2}+\left(\mathrm{d} \varepsilon_{2}^{\mathrm{P}}-\mathrm{d} \varepsilon_{3}^{\mathrm{P}}\right)^{2}\right. \\
\left.+\left(\mathrm{d} \varepsilon_{3}^{\mathrm{P}}-\mathrm{d} \varepsilon_{1}^{\mathrm{P}}\right)^{2}\right]^{1 / 2}
\end{gathered}
$$

where the $d E_{1}^{P}$ are the plastic strain components in principle coordinates. Experimental results indicate that at pressures on the order of the yield stress, plastic deformation occurs with no change in volume, which implies that

$$
d \varepsilon_{1}^{P}+d \varepsilon_{2}^{P}+d \varepsilon_{3}^{P}=0
$$

Therefore, in a uniaxial test with $\sigma_{1}=\sigma, \sigma_{2}=\sigma_{3}=0$, the plastic strain increments are

$$
d \varepsilon_{2}^{P}=d \varepsilon_{3}^{P}=-1 / 2 d \varepsilon_{1}^{P}
$$


Tnerefore in a uniaxial test, Equations (56) and (58) reduce to

$$
\sigma_{\mathrm{e}}=\sigma
$$

$$
d \varepsilon^{P}=d E_{1}^{P}
$$

Ihus, when the assumption is made that the yield stress is a function of the total effective plastic strain lcalled the strain Haraening hypotnesisl, the functional relationship between yield stress ano plastic strain can be taken directiy from a uniaxial stress-strdin curve by virtue of Equations $(61)$ and $(62)$.

Ine relationstic between the magnitudes of the plastic strain increments and the effective plastic strain increment is froviceo by the Pranoti-Reuss flow Rule:

$$
d \varepsilon_{i}^{P}=\frac{3 d \varepsilon^{P}}{2 \sigma_{e}} \quad s_{i} \quad i=1,2,3
$$

where the $S_{i}$ values are the deviatoric stress components in principal coordinates) detined by

$$
s_{i}=\sigma_{i}-\frac{1}{3}\left(\sigma_{1}+\sigma_{2}+\sigma_{3}\right) \quad i=1,2,3
$$

Equation (63) emtocies the fundamental observation of plastic deformation; that is, plastic strain increments are proportional to the ceviatoric stresses. The constant of proportionality is determined by the choice of the yield function. ${ }^{24}$ Direct substitution shows that Equations $(56),(58),(63)$, and $(64)$ are 
consistent with one another.

ünce the plastic strain increments have been determined for a given load step, the total strains are determined from a generalized formi of hooke's law given by

$$
\begin{aligned}
& \varepsilon_{I}=\frac{1}{E}\left\{\sigma_{1}-v\left(\sigma_{2}+\sigma_{3}\right)\right\}+\varepsilon_{I}^{P}+d_{\varepsilon_{I}}^{P}+\int a_{I} \mathrm{dI} \\
& \varepsilon_{2}=\frac{1}{E}\left\{\sigma_{2}-v\left(\sigma_{1}+\sigma_{3}\right)\right\}+\varepsilon_{2}^{P}+d \varepsilon_{2}^{P}+\int \alpha_{2} \mathrm{dT} \\
& \varepsilon_{3}=\frac{1}{E}\left\{\sigma_{3}-v\left(\sigma_{2}+\sigma_{1}\right)\right\}+\varepsilon_{3}^{P}+d_{\varepsilon_{3}}^{P}+\int \alpha_{3} \mathrm{~d} I
\end{aligned}
$$

in wich $E_{1}^{P}, \varepsilon_{2}^{P}$, and $\varepsilon_{3}^{p}$ are the total plastic strain components at the eno of the previous load increment.

The remaining continuum field equations of equilibrium, strain displacement, and strain compatibllity are unchanged. The complete set of governing equations is presented in Table 1 , written in terms of rectanguiar cartesian coordinates and employing the usual indicial notation in which a repeated latin index implies summation. This set of equations is augmented by an experimentally determined uniaxial stress-strain relation.

The Method of Solution-- when the problem under conslderation is staticaliy determinate so that stresses can be found from equiliorium conditions alone, the resulting plastic deformation can be determined directly. However, when the problem is statically indeterminate and the stresses and deformation must be found simulataneously, the full set of plasticity equations proves to be quite formidable, even in the case of simple loadings ano 
TABLE 1. SUMIARY OF GOVERNING EQUATIONS

Equilibrium

$$
\begin{aligned}
\sigma_{j i, j}+\rho f_{i} & =0 \\
\text { where } \sigma & =\text { stress tensor } \\
\rho & =\text { mass density } \\
f_{j} & =\text { components of body force per unit mass }
\end{aligned}
$$

Stress Strain

$$
\begin{aligned}
& \varepsilon_{i j}=\frac{l+v}{E} \sigma_{i j}-\delta_{i j}\left(\frac{v}{E} \sigma_{k k}-\int \alpha d T\right) \\
&+\varepsilon_{i j}^{p}+d \varepsilon_{i h}^{p}
\end{aligned}
$$

Compatibility

$$
\begin{gathered}
\varepsilon_{i j, k \ell}+\varepsilon_{k \ell, i j}-\varepsilon_{i k, j \ell}-\varepsilon_{j \ell, i k}=0 \\
\text { Definitions Used in Plasticity } \\
\sigma_{e} \triangleq \sqrt{\frac{3}{2} S_{i j} S_{i j}} \\
s_{j j} \triangleq \sigma_{i j}-\frac{1}{3} \sigma_{k k} \\
d_{\varepsilon}^{P} \triangleq \sqrt{\frac{2}{3} d \varepsilon_{i j}^{P}{ }_{d \varepsilon_{i j}^{P}}^{P}}
\end{gathered}
$$

Prandt1-Reuss Flow Rule

$$
d \varepsilon_{i j}^{P}=\frac{3}{2} \frac{d_{E}^{P}}{\sigma_{e}} \quad S_{i j}
$$

61 
geometries.

Une numerical procedure which has been used with consioerable success is the Method of Successive Substitutions. This method can be dpplied to any problem for which an elastic solution can be obtained, either in closed form or numerically. A full discussion of this technique, including a number of technologically useful examples, is contained in Reference 24 .

Griefly, the method involves breaking the loading path up into a number of smatl increments. For example, in the present application, the loads are external pressure, temperature, and either internal fressure or a prescribed displacement of the inside surface of the cladoing. These loads all vary during the operating history of the fuel rod. For each new increment of the loading, the solution to all the plasticity equations listed in Table 1 is obtained as follows.

First, an initial estimate of the plastic strain increments, $d E_{i j}^{p}$, is made. on the basis of these values, the equations of equilibrium, Hooke's lan, and strain-displacement and compatibility [Equations (119), (121), (125) and (126)] are solved as for any elastic protiem. From the stresses so obtained, the deviatoric stresses, $S_{i j}$, may be computed. This "pseudo-elastic" solution represents one path in the computational scheme.

Independentiy, through use of the assumed ${ }^{P}{ }_{i j}$ values, the

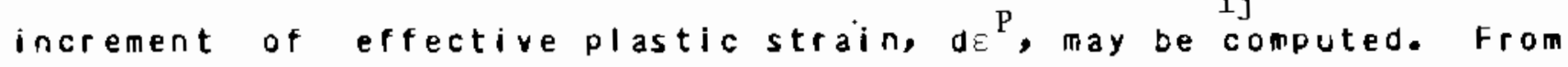
this result and the stress-strain curve, a value of the effective stress, o, is obtained. 
Finally, a new estimate of the plastic strain increments is ootained from the prandtl-Reuss flow rule

$d E_{i j}^{P}=\frac{3}{2} \frac{d \varepsilon^{P}}{\sigma_{e}} S_{i j}$

and the entire process is continued until the $d_{i j}$ converge. A schematic of the iteration scheme is shown in Figure 10 .

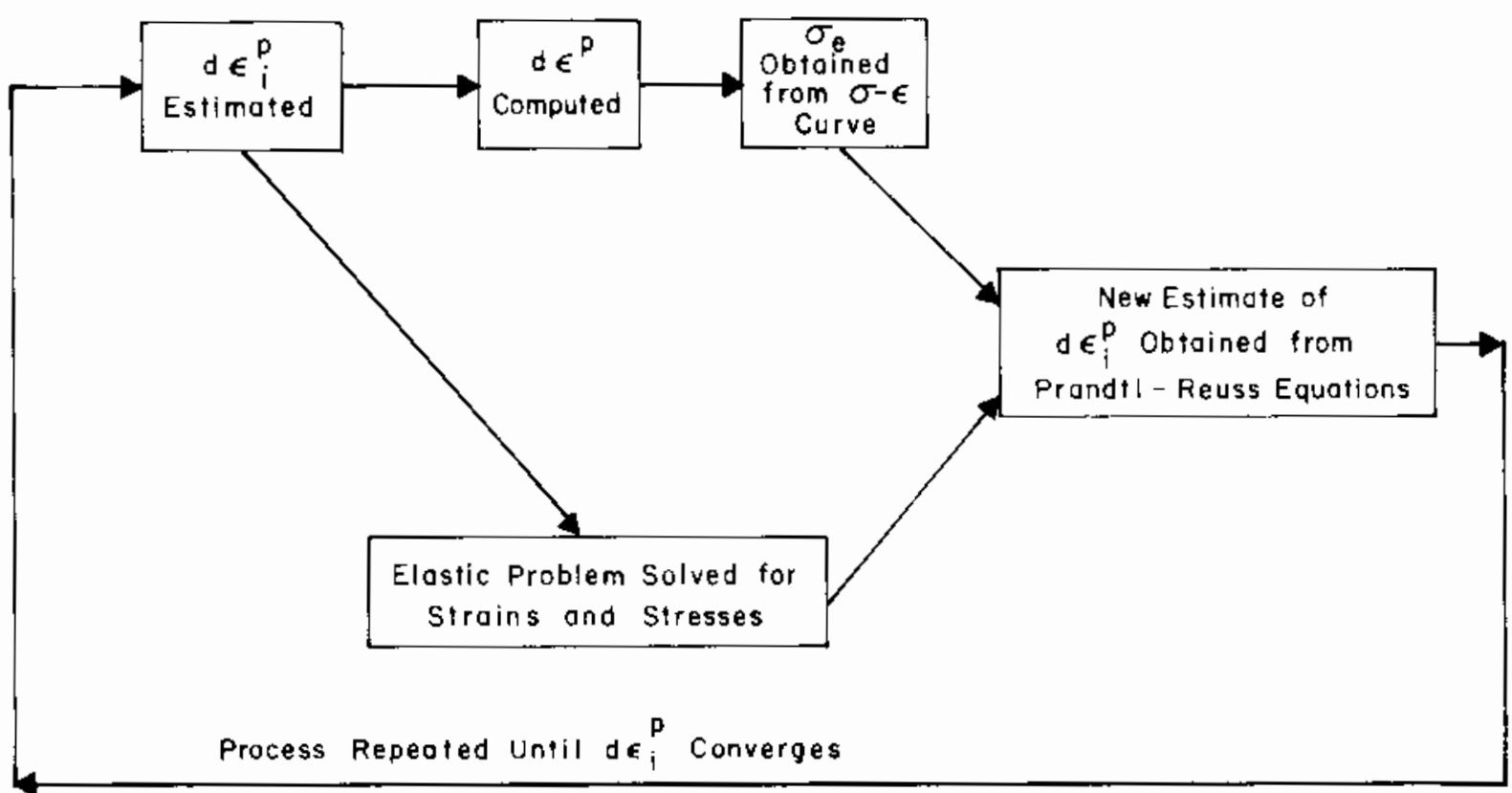

INEL-A-2162

Figure 10. Schematic of the method of successive elastic solutions. 
The mechanism by which improved estimates of ${ }^{P} \varepsilon_{i j}$ are ottained results from the fact that the effective stress obtained from $e^{P}$ and the stress-strain curve will not be equal to the effective stress that would be obtained with the stresses from the elastic solution. The effective stresses will only agree when convergence is obtained.

The question of convergence is one that cannot, in general, be answered a obtained for sufficiently small loadincrements. Experience has shown that this technique is suitable for both steady state and transient fuel roc analyses.

Extension to Creep and Hot-pressing--The methoo of solution descrited for the time-independent plasticity calculations can also be used for time-dependent creep and hot-pressing calculations. In this context, the term creep refers to any time-dependent constant volume permanent deformation, whereas the term hot-pressing refers to any time-dependent process which results in a permanent change in volume. Both creep and hot-pressing are stress-driven processes and are usually highly depenoent on temperature.

The only change required to extend the Method of Successive Elastic Solutions to allow consideration of creep and hot-pressing is to rewrite the prandti-Reuss flow rule [Equation (63)] as 


$$
\begin{aligned}
& \mathrm{d} \varepsilon_{1}^{c}=1.5 \frac{\dot{\varepsilon}^{c} \Delta t}{\sigma_{e}} s_{1}+\frac{\dot{v}^{c} \Delta t}{9} \frac{\left(\sigma_{1}+\sigma_{2}+\sigma_{3}\right)}{\sigma_{m}} \\
& \mathrm{~d} \varepsilon_{2}^{\mathrm{c}}=1.5 \frac{\dot{\epsilon}^{\mathrm{c}} \Delta t}{\sigma_{\mathrm{e}}} \mathrm{s}_{2}+\frac{\dot{\mathrm{v}}^{\mathrm{c} \Delta t}}{9} \frac{\left(\sigma_{1}+\sigma_{2}+\sigma_{3}\right)}{\sigma_{\mathrm{m}}} \\
& \mathrm{d} \varepsilon_{3}^{\mathrm{c}}=1.5 \frac{\dot{\epsilon}^{\mathrm{c}} \Delta \mathrm{t}}{\sigma_{\mathrm{e}}} \mathrm{s}_{3}+\frac{\dot{\mathrm{v}^{\mathrm{c}} \Delta \mathrm{t}}}{9} \frac{\left(\sigma_{1}+\sigma_{2}+\sigma_{3}\right)}{\sigma_{\mathrm{m}}}
\end{aligned}
$$

The first term on the right hand side of each of these equations computes the constant volume creep strain, whereas the second term in each equation computes the permanent change in volume. To use this form of che flow rule, two additonal material property correlations must be available. The first is a corretetion for constant volume creep strain, $\varepsilon^{c}$ (taken in a uniaxial test), as a function of stross, time, temperature, ano neutron flux; that is,

$$
E^{c}=f(\sigma, T, t, \dot{F})
$$

where

$\begin{aligned} \text { o } & =\text { uniaxial stress (MPa) } \\ T \quad & \text { temperature }(K) \\ t & =t i m e(s)\end{aligned}$


In the fkACAS madels, the strain hardening hypothesis is assumed, wich implies that the creep strain correlation can be differentiateo and solved for creep strain rate in the form

$$
\dot{\varepsilon}^{c}=h\left(\sigma, \varepsilon^{c}, T, \dot{F}\right)
$$

*nich is no tonger an explicit function of time. This equatior is obtained from the rATPRU package during the creep calculations.

The second aditional correlation required is a relationship between the rate of permanent volumetric strain and the applied loads; chat is,

$$
\dot{v}^{c}=g\left(\sigma_{m}, T, t, v_{\text {avail }}\right)
$$

where

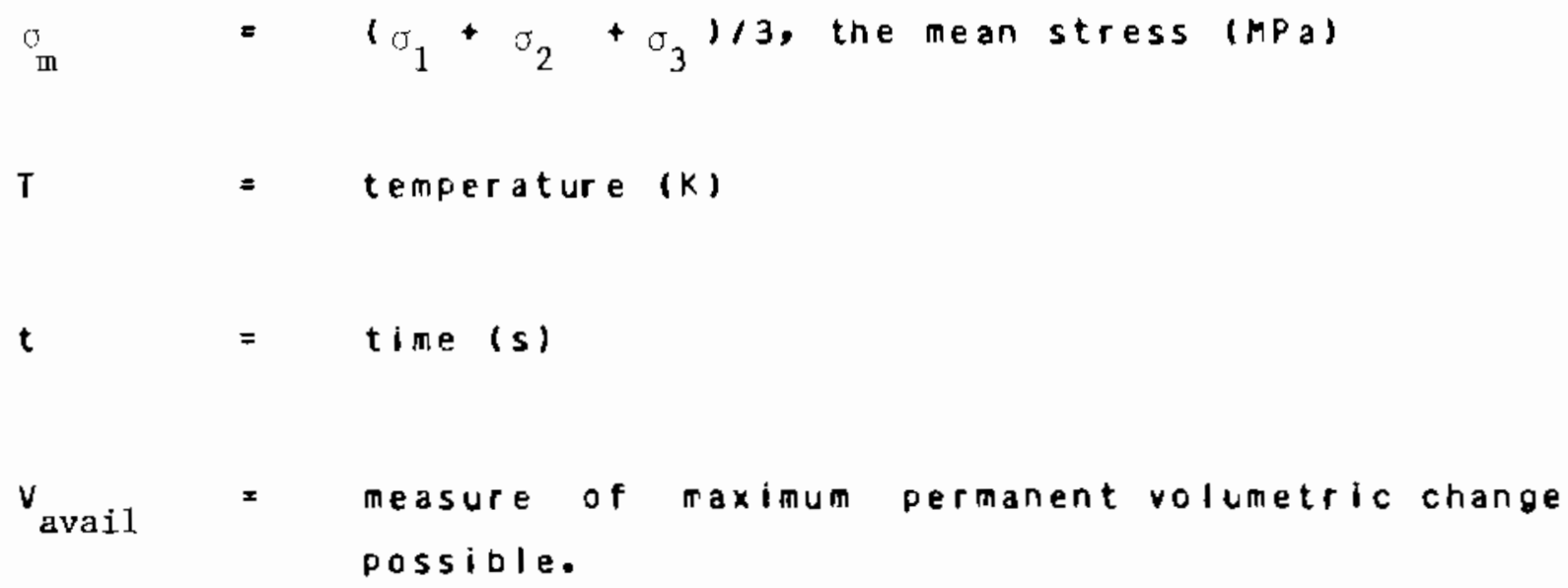


The permanent volumetric strain increment $d V^{c}$ is related to the creep strain increments by the equation

$$
d v^{c}=d \varepsilon_{1}^{c}+d \varepsilon_{2}^{c}+d \varepsilon_{3}^{c}
$$

In rkACAS-II, hot-fressing is considered only in the fuel. The source of the permanent volume change is assumed to be the healing of cracks in the relocated fuel. The maximum amount of volume available for permanent volume change is thus the amount of volume generated by fuel relocation. The equation for the permanent volume change was generated by comparing FRACAS-II calculated and measured Iength changes for experimental fuel rods irradiated in the power burst Facility and the Halden Test Reactor. The correlation which resulted in the test agreement with measured fuel rod length changes was found to be

$$
\Delta V=-V\left[1-\exp \left(-A \sigma^{B} \Delta t\right)\right]
$$

where

$\Delta v \quad=\quad$ rate of volume change $\left(m^{3} / s\right)$

A

$$
=1.0 \times 10^{-17}
$$

$=$ fuel-clading interface pressure (MPa)

$v \quad=$ relocation volume remaining $\left(m^{3}\right)$

$\Delta t \quad=\quad$ time stepsize $(s)$ 


$$
\text { B } \quad=4.5
$$

The relocation displacement for the oeformable pellet model (FRACAS-II) is computed by the equation

$U_{x}=\alpha \delta-\beta$

where

$\begin{aligned} & =\text { as-fatricated fuel-cladoing gap size }(\mathrm{m}) \\ \alpha & =0.79 \\ \beta & =0.0334(\mathrm{~m})\end{aligned}$

This equation for relocation displacement is based on the assumption that the fuel has not been subjected to repeated power cycles, as does the equation for relocation displacement discussed under the neading "fuel surface relocation" in section 2.4.1.2. No positive permanent volume change is permitted, and as the volumetric strain $v^{c}$ approaches the volume available from relocation, $V$ reloc, the permanent voluretric strain rate goes to zero.

As previously noted, two fRACAS models are available for analyzing the small deformation of the fuel and cladding. The first model cansiders the fuel pellets to be essentially rigld and to deform due to thermal expansion, swelling, and densification only. Thus, in the rigld pelfet model, the displacement of the fuel is calculated independently of the deformation of the 
cladding. This rigid pellet analysis is performed with the FRACAS-I subcode.

The second model available for the small deformation analysis is a more general analysis in which the fuel is assumed to deform due to stress, anc in this case the deformation of the fuel and cladoing must be determined simuttaneousiy. This deformable pellet analysis is performed with the fRACAS-II subcode.

The code user has the option of choosing either the rigia peliet or deformable pellet model. In general, the rigid pellet model (FRACAS-I) is less time-consuming and has proven to be adequate for a wide variety of reactor analysis in which pelletcladoing interaction is not the dominant fajlure mechanism. When pellet-cladding mechanical interaction is anticipated, however, the deformable pellet model (FRACAS-II) provides a more accurate calculation.

$$
\text { 2.4.1.2 Rigid Peliet Cladding Deformation Model--FRACAS-I }
$$
consists of a claoging deformation model and a fuel deformation model. If the fuel-cladding gap is closed, the fuel deformation model will apply a oriving farce to the clading deformation model. The cladding deformation model, however, never influences the fuel deformation model.

The clading deformation model in FRACAS-I is based on the following assumptions:

1. Incremental theory of piasticity 
2. Prandt-keuss flow rule

3. Isotropic work-hardening

4. No creep aeformation of cladding

3. Thin wall cladding lstress, strain, and temperature unitorm. through cladding thickness)

6. If fuel and cladoing are in contact, no axial slippage occurs at fuel cladding interface

7. Bending strains and stresses in cladding are negligible

8. Axisymmetric loading and deformation of cladoing.

The fuel deformation model in FRACAS-I is based on the following assumptions:

1. Thermal expansion, swelling, and densification are the only sources for fuel deformation

2. No resistance to expansion of tuel

3. No creep defortation of fuel 


\section{Isotropic tuel properties.}

The cladoing and fuel deformation models in FRACAS-I are described Delow.

Cladoing veformation Mocel-The rigid pellet cladding offormation subcode (FRACAS-I) consists of four sets of models, each used independently of the others.

Deformation and stresses in the cladoing in the open gap regime are computed using a model which considers a thin cylindrical shell with specified internal and external pressures ano a prescribed uniform: temperature.

Calculations for the closea gap regime are made using a model wich considers a thin cylindrical shell with prescribed external pressure and a prescribed radial displacement of the clading inside surface. The prescribed displacement is abtained from the fuel Expansion models described later in this section. Further, since no slippase is assumed to take place when the fuel and cladding are in contact, the axial expansion of the fuel is transmitted oirectly to the cladoing, and hence, the change in axial strain in the shell is also prescribed.

The decision whether the fuel-cladding gap is open or closed is made ty considering the relative movement of the cladding inside surface and the fuel outside surface. At the completion of the FRACAS-I analysis, either a new fuel-cladding gap size or a new fuel-cladding interfacial pressure and the elastic-plastic clading stresses and strains are obtained. 
Two additional models are used to compute changes in yield stress with work-hardening, given a uniaxial stress-strain curve. This stress-strain curve is obtained from MATPRO. The first model computes the effective total strain ano new effective plastic strain, given a value of effective stress and the effective plastic strain at the end of the last loading increment. The second model computes the effective stress, given an increment of plastic strain and the effective plastic strain at the end of the last loaoing increment. Depending on the work-hardened value of yletd stress, loading can be either elastic or plastic, and unloading is constrained to occur elastically. IIsotropic work-hardening is assumed in these calculationsl. These four sets of models are describea telow.

The decision as to whether or not the fuel is in contact with the cladding is made by comparing the radial displacement of the fuel surface with the radal displacement that nould accur in the clading due to the prescribed external (coolant) pressure and the prescribed internal (fission and fill gas) pressure. The free radial displacement of the cladding is obtained using equation (65). The following expression is used to decide if fuel-cladoing contact has occured:

$u_{\tau}^{\text {fuel }} \geq u_{I}^{c l a d}+\delta$

where

$$
\delta \quad=\text { as-fabricated fuel-cladoing gap size (m). }
$$

If equation (74) is satisfied, the fuel is in contactwith the cladding. The loading history enters into this decision by virtue 
of the permanent plastic cladding strains which are applied to the as-fabricated geometry. These plastic strains, ind total effective plastic strain, $E^{p}$, are retained for use in subsequent calculations.

If the fuel and cladding displacements are such that Equation (74) is not satisfied, the fuel-cladding gap has not closed during the current step and the solution obtained by the open gap solution is appropiate. The current value of the fuel-clading gap size is then computed and is used in the temperature calculations. The plastic strain values may be changed in the solution if adotional plastic straining has occurred.

If Equation (74) is satisfied, however, fuel and cladding contact has occurred during the current loading increment. At the contact interface, radial continuity requires that

$$
u_{r}^{c l a d}=u_{r}^{\text {fuel }}-\delta
$$

while in the axial direction the assumption is made that no slippage occurs between the fuel and the cladding. This state is referred to as "lockup".

Note that only the additional strain which occurs in the fuel after lock-up has occurred is transferred to the cladding. Thus, if $E_{\substack{z, 0 \\ \text { lad }}}$ is the axial strain in the cladoino just prior to contact, and

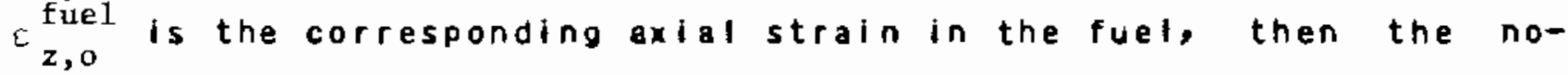
slippage condition in the axial direction becomes

$$
\varepsilon_{z}^{c l a d}-\varepsilon_{z, 0}^{c l a d}=\varepsilon_{z}^{\text {fue } 1}-\varepsilon_{z, 0}^{\text {fuel }}
$$


The values of the "prestrains", $\varepsilon_{z, 0}^{f u e l}$ and $E_{z, 0}^{c l a d}$, are set equal to the values of the strains that existed in the fuel and cladoing at the time of fuel-claoding gap closure and are stored and used in the cladcing sequence of calculations. The values are updated at the enu of any load increment ouring which the fuel-cladoing gap is closed.

After $u=c$ and $\varepsilon_{z}^{c l a d}$ have been computed, they are used in a calculation which considers a thin cylindrical shell with prescribed axiad strain, external pressure and prescribedradial displacement of the inside surface. After the solution is obtained, a value of the fuel-clading interfacial pressure is computed along with nem plastic strains and stresses.

The open gaf nodeling considers a thin cylindrical shell loaded by both internal ano external pressures. Axisymmetric loading and oeformation are assumed. Loading is also restricted to being uniforn in the axial cirection, and no bending is considered. The geometry and coordinates are shown in figure 11. The displacements of the mioplane of the shell are u and w in the radial and axial directions, respectively.

For this case, the equilitiom equations are identically satisfied by

$\sigma_{\theta}=\frac{r_{i} P_{i}-r_{0} P_{0}}{t}$

$\sigma_{z}=\frac{r_{i}^{2} P_{i}-r_{o}^{2} P_{o}}{r_{n}^{2}-r_{i}^{2}}$

(78) 

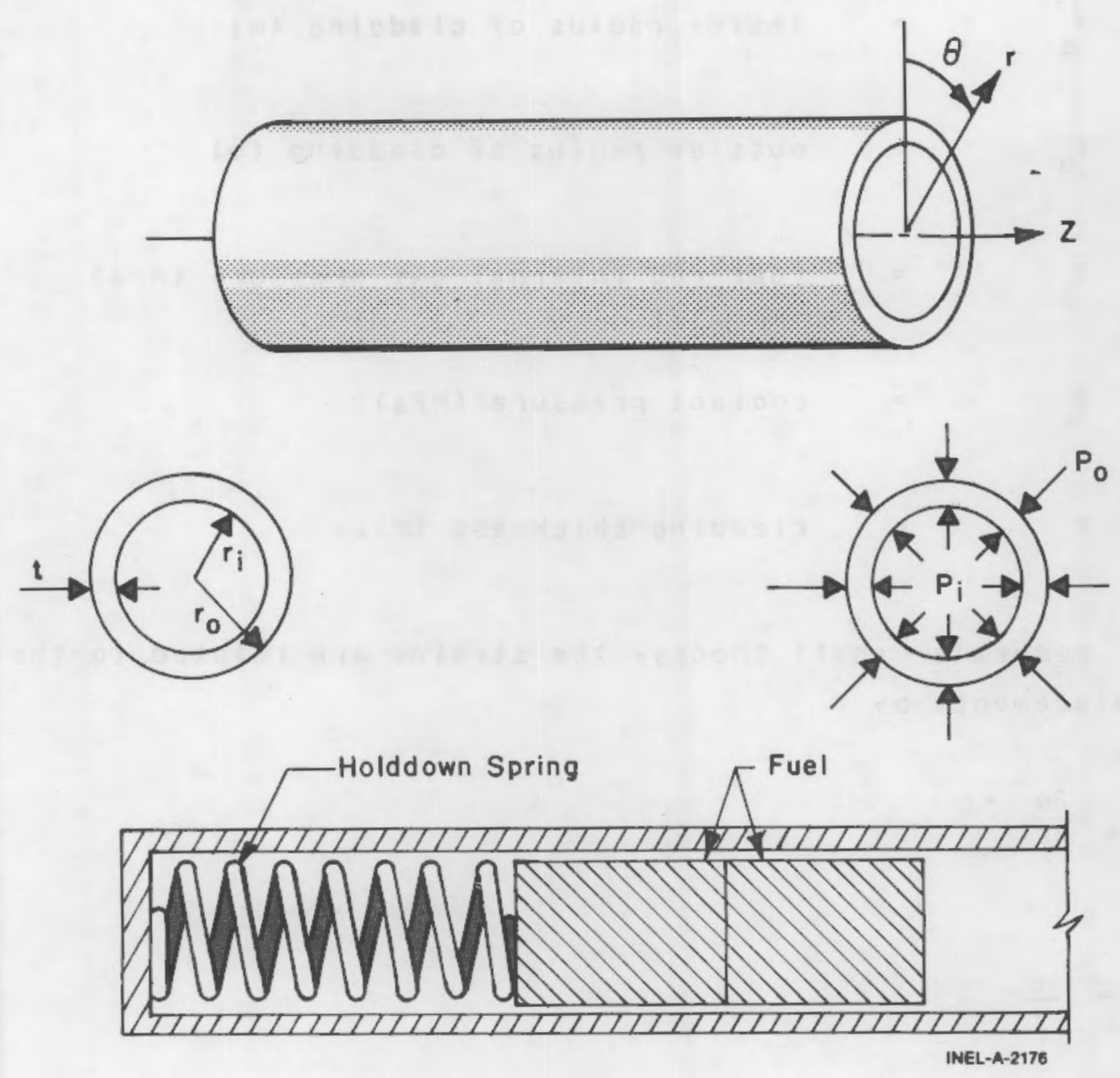

Figure 11. Fuel rod geometry and coordinates.

where

$\begin{aligned} \sigma_{\theta} & =\text { hoop stress (MPa) } \\ \sigma_{z} & =\text { axial stress (MPa) }\end{aligned}$



$r_{i} \quad=\quad$ inside radius of cladoing $(\mathrm{m})$
ro outside radius of cladding (m)
$P_{i} \quad=\quad$ fuel rod internal gas pressure (MPa)
$P_{0} \quad=\quad$ coolant pressure (MPa)
t $=$ cladding thickness $(\mathrm{m})$.

For membrane shell theory, the strains are related to the midplane displacements by

$$
\begin{aligned}
& \varepsilon_{z}=\frac{\partial w}{\partial z} \\
& \varepsilon_{\theta}=\frac{u}{\vec{r}}
\end{aligned}
$$

where $\bar{r}$ is the radius of the midplane. Strain across the thickness of the shell is allowed. In shell theory, since the radial stress can be neglecteo, and since the hoop stress, $\sigma_{\theta}$, and axial stress, $\sigma_{z}$, are uniform across the thickness when bending is not considered, the radial strain is due only to the Poisson's effect and is uniform across the thickness. INormally, radial strains are not considered in a shell theory, but plastic radial strains must be included when plastic deformations are considered).

The stress-strain relations are written in incremental form as 


$$
\begin{aligned}
& \varepsilon_{\theta}=\frac{1}{E}\left\{\sigma_{\theta}-v \sigma_{z}\right\}+\varepsilon_{\theta}^{P}+d \varepsilon_{\theta}^{P}+\int_{T_{0}}^{T} \alpha_{\theta} d T \\
& \varepsilon_{z}=\frac{1}{E}\left\{\sigma_{z}-v \sigma_{\theta}\right\}+\varepsilon_{z}^{P}+d \varepsilon_{z}^{P}+\int_{T_{0}}^{T} \alpha_{z} d T \\
& \varepsilon_{r}=-\frac{v}{E}\left\{\sigma_{\theta}+\sigma_{z}\right\}+\varepsilon_{r}^{P}+d \varepsilon_{r}^{P}+\int_{T_{0}}^{T} \alpha_{r} d T
\end{aligned}
$$

where

$$
\begin{aligned}
T_{0} & =\text { strain-free reference temperature }(K) \\
\alpha \quad & =\text { coefficient of thermal expansion } \\
T \quad & \text { current average cladding temperature }(K) \\
E \quad & \text { modulus of elasticity } \\
& =\text { Poisson's ratio. }
\end{aligned}
$$

The terms $\varepsilon_{\theta}^{P}, \varepsilon_{z}^{P}$ and $\varepsilon_{r}^{P}$ are the plastic strains at the end of the last load increment, and $d \varepsilon_{\theta}^{P}, d \varepsilon_{z}^{P}$, and $d \varepsilon_{r}^{P}$ are the additional plastic strain increments which occur due to the new load increment. 
The magnitude of the adoitional plastic strain increments is determined by the effective stress and the Prandtl-Reuss flow rule, expresses as

$$
\begin{aligned}
& \sigma_{e}=\frac{1}{\sqrt{2}}\left[\left(\sigma_{\theta}-\sigma_{z}\right)^{2}+\left(\sigma_{z}\right)^{2}+\left(\sigma_{\theta}\right)^{2}\right] 1 / 2 \\
& d \varepsilon_{i}^{P}=\frac{3}{2} \frac{d \varepsilon^{P}}{\sigma_{e}} s_{1} \text { for } i=r, \theta, z \\
& s_{i}=\sigma_{i}-\frac{1}{3}\left(\sigma_{\theta}+\sigma_{z}\right) \text { for } i=r, \theta, z
\end{aligned}
$$

The solution of the open gap case proceeds as follows. At the end of the last loao increment the plastic strain components, $\varepsilon_{\theta_{P}}^{P}, E_{z}^{P}$, and $\varepsilon_{r}^{p}$ are known. also the total effective plastic strain, $E^{P}$, is known.

The loaoing is now incremented with the prescribed values of $P_{i}, P_{0}$, and $T$. The new stresses can be determined from Equations (77) and (78), anc a new value of effective stress is obtained from Equation $(84)$.

The increment of effective plastic strain, de , which results from the current increment of loading, can now be determined from the uniaxial stress-strain curve at the new value of $\sigma_{e}$, as shom in Figure 12. (The new elastic loading curve depends on the value of $\varepsilon$ ).

Once de $E^{P}$ is determined, the individual plastic strain components are found from Equation (85), and the total strain 


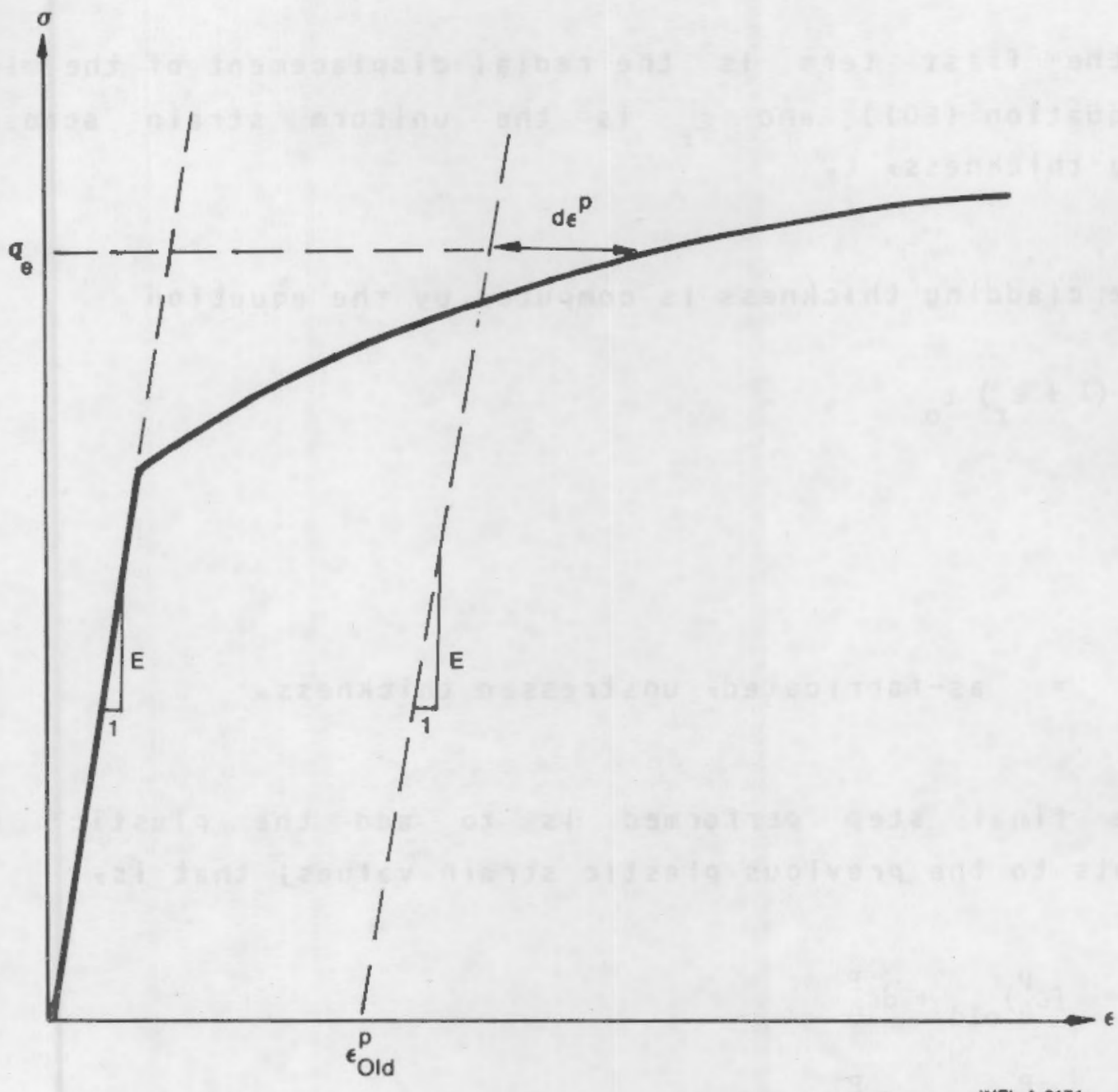

INEL-A-2174

Figure 12. Calculation of effective stress $\sigma_{e}$ from $d \varepsilon^{P}$.

components are obtained from Equations (81) thorugh (83).

The displacement of the inside surface of the shell must be determined so that a new fuel-cladding gap width can be computed. The radial displacement of the inside surface is given by

$u\left(r_{1}\right)=\bar{r} \varepsilon_{\theta}-\frac{t}{2} \varepsilon_{r}$ 
where the first term is the radial displacement of the midplane [from Equation ( 80$)$ ] and $\varepsilon_{r}$ is the uniform strain across the cladding thickness, t.

The cladding thickness is computed by the equation

$t=\left(1+\varepsilon_{\mathbf{r}}\right) t_{0}$

$(88)$

where

$t_{0}=$ as-fabricated, unstressed thickness.

The final step performed is to add the plastic strain increments to the previous olastic strain values; that is,

$\left(\varepsilon_{\theta}^{\mathbf{P}}\right)_{\text {new }}=\left(\varepsilon_{\theta}^{\mathbf{P}}\right)_{\text {old }}+\mathrm{d} \varepsilon_{\theta}^{\mathbf{P}}$

$\left(\varepsilon_{z}^{P}\right)_{\text {new }}=\left(\varepsilon_{z}^{P}\right)_{\text {old }}+d \varepsilon_{z}^{P}$

$\left(\varepsilon_{\mathbf{r}}^{\mathrm{P}}\right)_{\text {new }}=\left(\varepsilon_{\mathbf{r}}^{\mathrm{P}}\right)_{\text {old }}+\mathrm{d} \varepsilon_{\mathbf{r}}^{\mathrm{P}}$

$\left(\varepsilon^{P}\right)_{\text {new }}=\left(\varepsilon^{P}\right)_{\text {old }}+d \varepsilon^{P}$

These values are used in or for the next load increment.

Thus all the stresses and strains can be computed directly, since in this case the stresses are determinate. In the case of the driven cladding displacement, the stresses depend on the displacement, and such a straightforward solution is not possible. 
The closed gap modeling considers the problem of a cylindrical shell for which the radial displacement of the inside surface and axial strain are prescribed. Here the stresses cannot be computed directly since the pressure at the inside surface t the fuel-cladaing interfacial pressurel must be determined as part of the solution.

As in the open gap modeling, the displacement at the inside surface is given by

$$
u\left(r_{i}\right)=u-\frac{t}{2} \varepsilon_{r}
$$

where $u$ is the radial displacement of the midplane. From Equation $(B 1), u=r E_{0}$ and

$$
u\left(r_{i}\right)=\tilde{r} \varepsilon_{\theta}-\frac{t}{2} \varepsilon_{r}
$$

Tnus, prescribing the displacement of the inside surface of the sheli is equivalent to a constraining relation between $\varepsilon_{\theta}$ and $E_{i}$. As before, Hooke's law is taken in the form

$$
\begin{aligned}
& \varepsilon_{\theta}=\frac{1}{E}\left(\sigma_{\theta}-v \sigma_{z}\right)+\varepsilon_{\theta}^{P}+d \varepsilon_{\theta}^{P}+\int_{T_{0}}^{T} \alpha_{\theta} d T \\
& \varepsilon_{z}=\frac{1}{E}\left(\sigma_{z}-v \sigma_{\theta}\right)+\varepsilon_{z}^{P}+d \varepsilon_{z}^{P}+\int_{L_{0}}^{T} \alpha_{z} d T
\end{aligned}
$$


$E_{r}=-\frac{v}{E}\left(\sigma_{\theta}+\sigma_{z}\right)+\varepsilon_{r}^{p}+d \varepsilon_{r}^{P}+\int_{r_{0}}^{\mathrm{T}} \rho_{r} \mathrm{dT}$

Use of Equation (91) and (94) in Equation (92) results in a relation between the stresses $\sigma_{\theta}, \sigma_{z}$, and the prescribed displacement u(r, ${ }_{i}$

$$
\begin{aligned}
& \frac{u\left(r_{i}\right)}{\bar{r}}+\frac{1}{2} \frac{\left(\frac{t}{r}\right)}{r}\left\{\varepsilon_{r}^{P}+d \varepsilon_{r}^{p}+\int_{T}^{T} \quad \alpha d T\right\} \\
& -\left\{\varepsilon_{\theta}^{P}+\mathrm{d} \varepsilon_{\theta}^{P}+\int_{T_{0}}^{T} \operatorname{dd} T\right\}=\frac{1}{E}\left[\left(1+\frac{\nu}{2} \frac{t}{\bar{r}}\right) \sigma_{\theta}\right. \\
& \left.+v\left(\frac{1}{2} \frac{t}{\bar{\tau}}-1\right) \sigma_{z}\right]
\end{aligned}
$$

Equations (93) and (95) are now a pair of simultaneous algabraic equations for the stresses $\sigma_{\theta}$ and $\sigma_{z}$, which may be written as

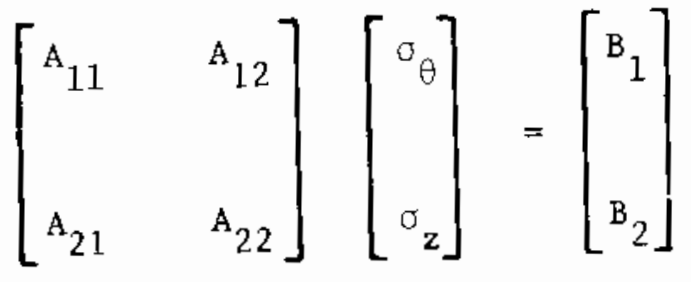

where

$$
\begin{aligned}
& A_{11}=1+\frac{v}{2} \frac{t}{r} \\
& A_{12}=v\left(\frac{1}{2} \frac{t}{r}-1\right) \\
& A_{21}=-v
\end{aligned}
$$




$$
\begin{aligned}
& \mathrm{A}_{22}=1 \\
& \mathrm{~B}_{1}=\frac{\mathrm{U}\left(\mathrm{r}_{\dot{i}}\right)}{\overline{\mathrm{r}}}+\frac{\mathrm{E}}{2} \underset{\frac{\mathrm{r}}{\mathrm{r}}}{\left(\frac{\mathrm{t}}{\mathrm{r}}\right)}\left\{\varepsilon_{\mathrm{r}}^{\mathrm{P}}+\mathrm{d} \mathrm{E}_{\mathrm{r}}^{\mathrm{P}}+\int_{\mathrm{T}_{0}}^{\mathrm{T}}\langle\mathrm{dT}\}\right. \\
& -E\left\{E_{\theta}^{\mathrm{P}}+\mathrm{d} \varepsilon_{\theta}^{\mathrm{P}}+\int_{\mathcal{T}_{0}}^{\mathrm{T}} \alpha \mathrm{dT}\right\} \\
& B_{2}=E\left[E_{z}-E \varepsilon_{z}^{P}+d \varepsilon_{z}^{P}+\int_{i_{0}}^{T} \alpha d T\right]
\end{aligned}
$$

Then the stresses can be written explicity as

$$
\begin{aligned}
& \sigma_{0}=\frac{\mathrm{B}_{1} \mathrm{~A}_{22}-\mathrm{B}_{2} \mathrm{~A}_{12}}{\mathrm{~A}_{11} \mathrm{~A}_{22}-\mathrm{A}_{12} \mathrm{~A}_{21}} \\
& \sigma_{z}=\frac{\mathrm{B}_{2} \mathrm{~A}_{11}-\mathrm{B}_{1} \mathrm{~A}_{21}}{\mathrm{~A}_{11} \mathrm{~A}_{22}-\mathrm{A}_{12} \mathrm{~A}_{21}}
\end{aligned}
$$

Inese equations relate the stresses to vir ${ }_{i}$ and $\varepsilon_{z}$, which are prescrioed, and to $d e_{\theta}^{P}$, $d \varepsilon_{z}^{P}$, and $d E_{r}^{P}$ which are to be determined. The remaining equations which must be satisfied are

$$
\begin{aligned}
& \sigma_{\mathbf{e}}=\frac{1}{\sqrt{2}}\left[\left(\sigma_{\theta}-\sigma_{z}\right)^{2}+\left(\sigma_{\theta}\right)^{2}+\left(\sigma_{z}\right)^{2}\right]^{1 / 2} \\
& d \varepsilon^{P}=\frac{2}{3}\left[\left(d_{r}^{P}-d \varepsilon_{\theta}^{P}\right)^{2}+\left(d \varepsilon_{\theta}^{P}-d \varepsilon_{z}^{P}\right)^{2}+\left(d \varepsilon_{z}^{P}-d \varepsilon_{r}^{P}\right)^{2}\right] 1 / 2
\end{aligned}
$$

and the Prandti-Reuss flow equations [defined in Equation (85)] 
${ }_{d} \varepsilon^{P}=\frac{3}{2} \frac{d \varepsilon^{P}}{\sigma_{e}}\left[\sigma_{\theta}-\frac{1}{3}\left(\sigma_{\theta}+\sigma_{z}\right)\right]$

$\mathrm{d} \varepsilon_{z}^{P}=\frac{3}{2} \frac{\mathrm{d} \varepsilon^{\mathrm{P}}}{\sigma_{\mathrm{e}}}\left[\sigma_{z}-\frac{1}{3}\left(\sigma_{\theta}+\mathrm{c}_{z}\right)\right]$

$\mathrm{d} \varepsilon_{r}^{\mathrm{P}}=-\mathrm{d} \varepsilon_{0}^{P}-\mathrm{d} \varepsilon_{z}^{P}$

The effective stress, ${ }^{\circ}{ }_{\theta}$, and the plastic strain increment, ${ }^{P}$, must of course, be related by the uniaxial stress-strain lah. Equations (97) through (103) must be simultaneously satisfied for each loading increment.

As aiscussed in Section 2.4.1.1, a straightorward numerical solution to these equations can be obtained by means of the method of Successive Elastic Solutions. By this method, arbitrary values are initially assumed for the increments of plastic strain, and Equations (97) through (103) are used to obtain improved estimates of the plastic strain components. The steps performed are as follows for each increment of load:

1. Values of $0 \varepsilon_{\theta}^{P}, d \epsilon_{z}^{P}$, and ${ }_{a} \varepsilon_{r}^{P}$ are assumed. Then, $d E^{P}$ is computed from Equation $(100)$ and the effective stress is obtained from the stress-strain curve at the value of.

2. From Hooke's law, still using the assumed plastic strain increments and the prescribed values of $u\left(r_{i}\right)$ and $\varepsilon_{z}$, values for the stresses can be obtained from Equations $(97)$ and $(98)$. 
3. New values for $d e_{a}^{P}, d c_{z}^{P}$, and $d{ }_{r}^{P}$ are now computed from the pranoti-Reuss reliations,

$\left.d \varepsilon_{i}^{P}=\frac{3}{2} \frac{d E^{P}}{e} 10_{i}-\frac{1}{3}\left(\sigma_{i}+o_{z}\right)\right] \quad i=r, 0,2$

using as conputeo in step is and as computed in step 2.

4. The old and nem values of $d e_{2}^{P}, d E_{2}^{P}$, and de $e_{r}^{P}$ are comparea and the process continued until convergence is obtaineo.

b. Unce convergence has been ootained, the fuel-cladaing interfacial pressure is computed from the following equation.

$P_{i n t}=\frac{t \sigma_{0}+r_{0} P_{0}}{r_{i}}$

when Steps I through 5 have teen accomplisheo, the solution is complete, provided that the fuel-cladoing interface pressure is not less than the tocal gas pressure.

However, due to unequal amounts of plastic straining in the hoop anc axial cirections upon unloading, the tuel-cladoing interfacial pressure as obtaineo in 5 tep 5 is often less than the gas pressure even though the fuel-cladding gap has not opened. when this situation occurs, the frictional "locking" iwhich is assumed to constrain the cladoing axial deformation to equal the fuel axial deformation) no longer exists. The axial strain and stress adjust themselves so that the tuel-cladoing interfacial pressure equals the 
gas pressure, at hhich point the axial strain is again "lockea". Thus, upon further unloading, the axial strain and the hoop ano axial stresses continually readjust themselves to maintain the fuel-cladoing interfacial pressure equal to the gas pressure until tre fuel-cladding gap opens. Since the unloading occurs elasticaliy, a solution for this portion of the fuel-cladoing interaction problen can be cotained directly as discussed below.

Since the external pressure and the fuel-caldding interfacial pressure are knokn, the hoop stress is obtained from Equation as

$\sigma_{\theta}=\frac{r_{i} P_{i n t}-r_{0} P_{0}}{t}$

From Equation (91), the following expression can be written

$\varepsilon_{0}=\frac{u_{r}^{\text {fuel }}-\delta+t / 2 \varepsilon_{r}}{\bar{r}}$

Substitution of $\varepsilon_{\theta}$ and $\varepsilon_{r}$, as given by Equations $(92)$ and $(94)$, into Equation (107) results in an explicit equation for $\sigma_{z}$ :

$$
\begin{aligned}
v r_{i} \sigma_{z} & =(\bar{r}+v t / 2) \sigma_{\theta}+\bar{r} E\left(\int \alpha d T+d \varepsilon_{\theta}^{P}\right) \\
& -\frac{t}{2} E\left(\int \alpha d T+d \varepsilon_{r}^{P}\right)-E u\left(r_{i}\right)
\end{aligned}
$$

in which $\sigma_{\theta}$ is knokn from Equation (106). With $\sigma_{z}$ and $\sigma_{\theta}$ known, the strains may be computed from Hooke's law, Equations (92) through 1941. This set of equations is automatically invoked whenever $P$ int 
is computed to be less than the local gas pressure.

As in the oper gap modeling, the last step performed is to set the plastic strain components and total effective strain equal to their new values by adding in the computed increments $d \varepsilon_{i}^{p}$ and $d e^{P}$.

The stress-strain modeling is used to relate stress and plastic strain, taking into consideration the dicection of loading and the previous plastic deformation. A typical stress-strain curve is shown in Figure 13. This curve presents the results of a uniaxial

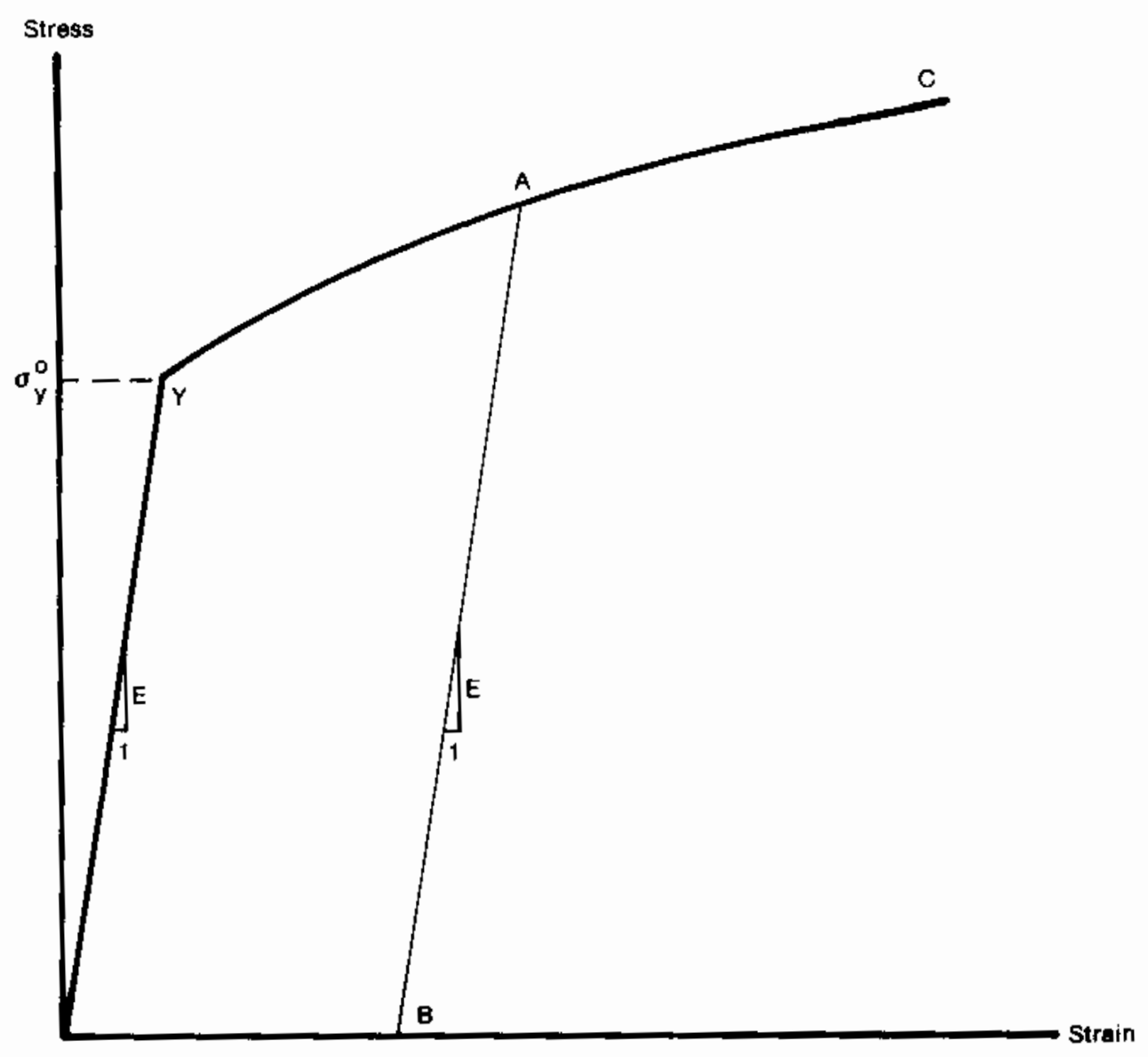

Figure 13. Idealized stress-strain behavior. 
stress-strain experiment and may be interpreteo beyond initial yielo as the locus of work-hardened yield stresses. The equaticn of the curve is provided ly the MATPRi fackage at each temperature.

To utilize this intormation, the usual idealization of the mecnanical benavior of metals is made. Inus, linear elastic behavior is assumec until a sharply defined yield stress is reached, dfter which plastic (irrecoverablel deformation occurs. unloading from a stress state beyond the initial yield stress, y, is assumed to occur along a straight line havirg the elastic modulus for its slope. wnen the (uniaxial) stress is removed completely, a residual plastic strain renains, anc this completely determines the suosequent yield stress. That is, when the speciren is loaded again, loading mill occur along line Ba in Figure 13 , ano no adjitionat deformation will occur until point $A$ is again reachec. Point $A$ is the subsequent yield stress. If $0=f(c)$ is the equation of thic plastic portion of the stress-strain curve (YAC), then for a given value of plastic strain, the subsequent yielo stress is found oy simultaneously solving the pair of equations

$$
\left\{\begin{array}{l}
G=f(\varepsilon) \\
\sigma=E\left(\varepsilon-\varepsilon^{P}\right)
\end{array}\right.
$$

which may be written as

$\sigma=\mathrm{f}\left(\frac{\sigma}{\mathrm{E}}+\mathrm{E}^{\mathrm{P}}\right)$

Inis nontinear equation may be solved efficiently by using Newton's iteration scheme 
$\sigma^{(m+1)}=E\left[\frac{\sigma^{(m)}}{E}+:{ }^{p}\right] \quad m=0,1,2, \ldots$.

Tne initial iterate, $\sigma^{(0)}$, is arbitrary, and without loss of jenerality, is taken as $34.5 \mathrm{MPa}$. For any monotonically increasing stress-plastic strain relaticn, the iteration scneme in Equation (111) will converge uniformy and absolutely.

The computations of the stress-strain modeling are described delom. The first computes strin as a function of plastic straine temperature, and stress. The second computes stress as a function of plastic strain, temperature and plastic strain increments.

values of plastic strain, $e^{p}$, temperature and stress are used as follows:

1. For a given temperature, 0 f(c) is obtainec from the MATPRO package.

2. The yield stress $C_{y}$ for given $E^{P}$ is obtained from Equation (111).

3. For a given value of stress,

$$
\text { if } \begin{gathered}
\bar{z} \sigma_{y}, \varepsilon=\frac{\sigma}{E}+\varepsilon^{P} \\
\varepsilon_{\text {new }}^{P}=\sigma_{\text {old }}^{p}
\end{gathered}
$$




$$
\begin{aligned}
& \text { if } \sigma>\sigma y, \varepsilon=E(\sigma) \\
& E_{\text {new }}^{P}=\varepsilon-\sigma / E \\
& { }_{d \varepsilon^{P}}^{P}=E_{\text {new }}^{P}-\varepsilon_{\text {old }}^{P} .
\end{aligned}
$$

where $E$ is computeo using the MATPRD package.

Values of plastic strain, $\varepsilon^{P}$, temperature, and plastic strain increment, de $\varepsilon^{P}$, are used as follows:

1. For a given temperature, $\sigma=f(\varepsilon)$ is obtained from the matpro gackage.

2. The yielo stress $\sigma_{y}$ for given $\varepsilon^{P}$ is ootained from Equation (111).

3. Given $d \epsilon^{P}$ (see Figure 14),

$\varepsilon_{\text {new }}^{P}=\varepsilon_{\text {old }}^{P}+d \varepsilon^{P}$

Since $d E^{P}>0$, the new value of stress and strain must lie on the plastic portion of the stress-strain curve $\sigma=f(E)$. So, $\sigma$ and Eare obtained by performing a simultaneous solution, as before.

Rigid Pellet Fuel Deformation in FRACAS-I--This section describes the analytical models used to compute fuel deformation in FRACAS-I. Models are arailable to calculate length change and fuel 


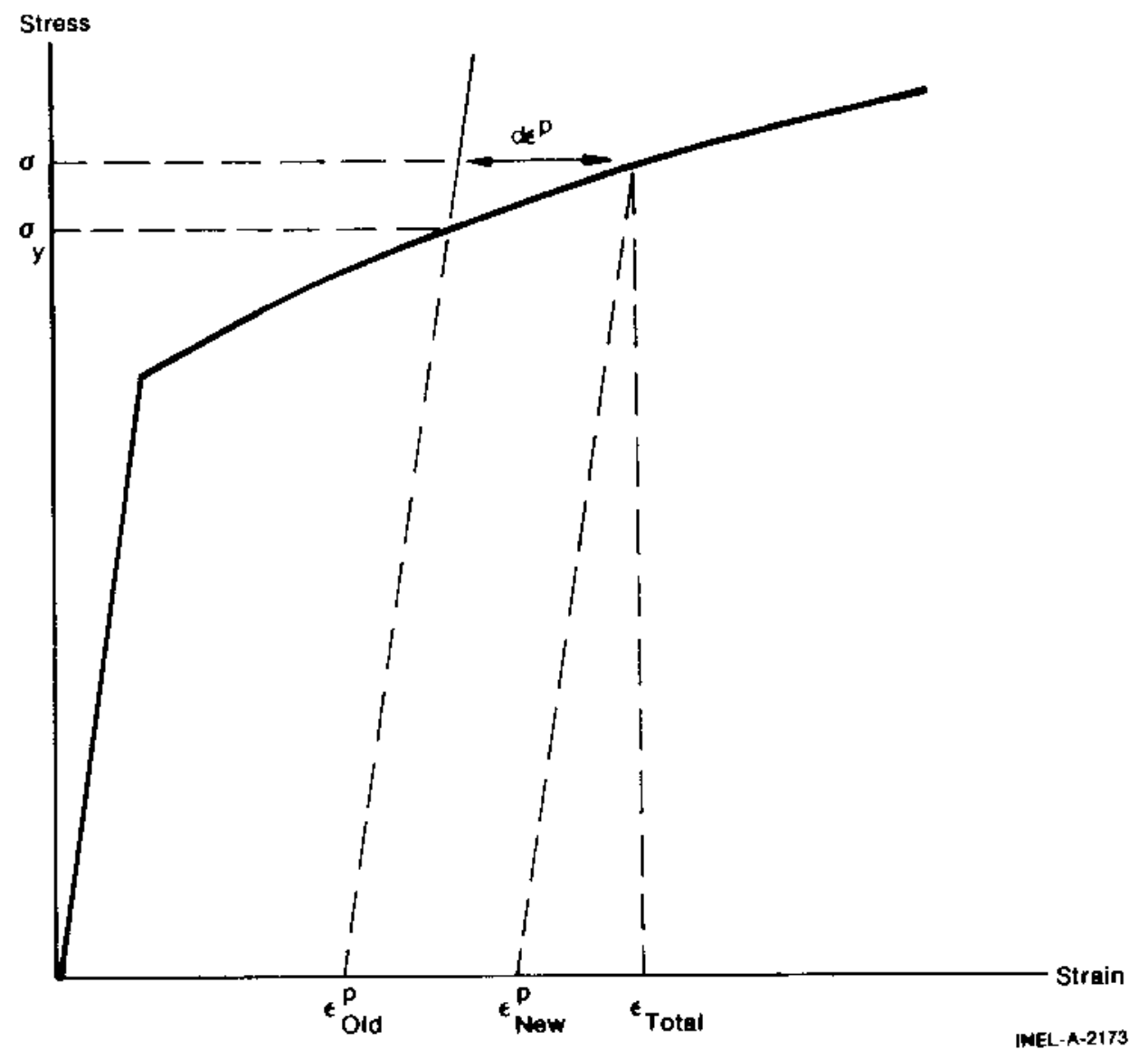

Figure 14. Computing STRESS.

radial displacement. Relocation is also considered in FRACAS-I, and is also discussed in this section. The effect of relocation. however, is incluaed only in the thermal response and is not consideredin the mechanical response of the fuel and cladding.

The assumptions made with respect to fuel deformation in FRACAS-I are that no pellet deformation is induced by fuel-cladding cantact stress or thermal stress and that freering thermal expansion applies. Each individual fuel ring is assumed to expand 
* ithout restraint fron any ctner ring, anc the total expansion is the sur of the inciviaual exfansions.

Rajial ueformation=-kadial oeformation of the pellet gue to thermal expansion, irrajiation shelling and oensification is calcuated with a tree-ring expansion model. The governing equation for tnis model is

$R_{H}=\sum_{i=1}^{N} r_{i}\left(1+\partial_{\Gamma_{i}} \therefore \Gamma_{i}+\cdots s_{i}+v_{i}\right)$

where

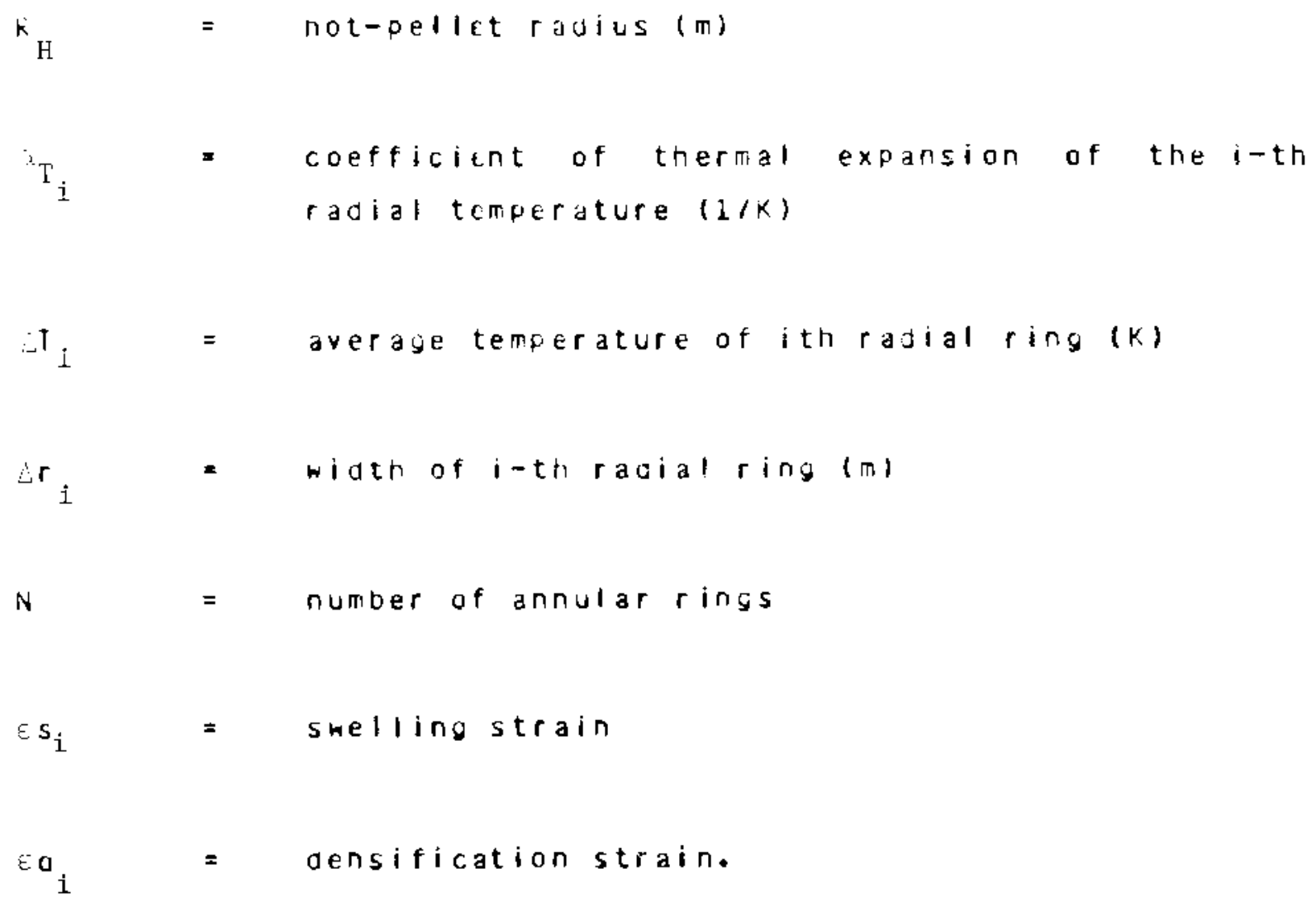


Axial deformation--Axial deformation of the fuel stack is calculated by sumping the maximum ring axial expansions of each pellet. Maximun ring axial expansion of each pellet is calculateo as the difference between the length of the ring with the maximum overall hot length and the cold length of that ring.

The calculation of the overall ring height includes consioeration of a central oish, wher present. The fuel stack length 15 this calculateo from

$L_{\bar{I}}=\sum_{j=I}^{M}\left(l+v_{i} \Delta T_{i}+c_{s}+s_{i}\right) d_{j} j$

where

- = the rins with maximum axial length of the juth node

$\mathrm{L}_{\mathrm{f}} \quad=$ not length of the fuel stack $(\mathrm{m})$

M number of axial nodes

$L_{i} \quad=$ length of the j-th radial ring (m).

Fuel Surface kelocation--Two closely related models are used to compute the effect of relocation. The fuet surface relocation model results in an effective pellet-cladding gap which is used in computing the gaf conductance and the thermal gap. The sface made arailable tor cracks based on this model, is distributed inside the 
peliet. This leads to the second relocation model which calculates an effective thermal conductivity across the cracked pellet. The effective thermal conductivity model was previously discussed in section 2.3 .7 .1 .

The amount of fuel surface relocation necessary to result in the proper gap closure versus power was studied by coleman using FRAP-T3. These results are presented in Reference 25. The model which was developed from these results provides FRACAS-I with an effective fuel strain which is a function of the fuel roo cold-state geometry. This strain is treated the same as the other fuel radial strains (thermal expansion, swelling, and densification). With reference to Figure 15 , the equation for the variable relocation

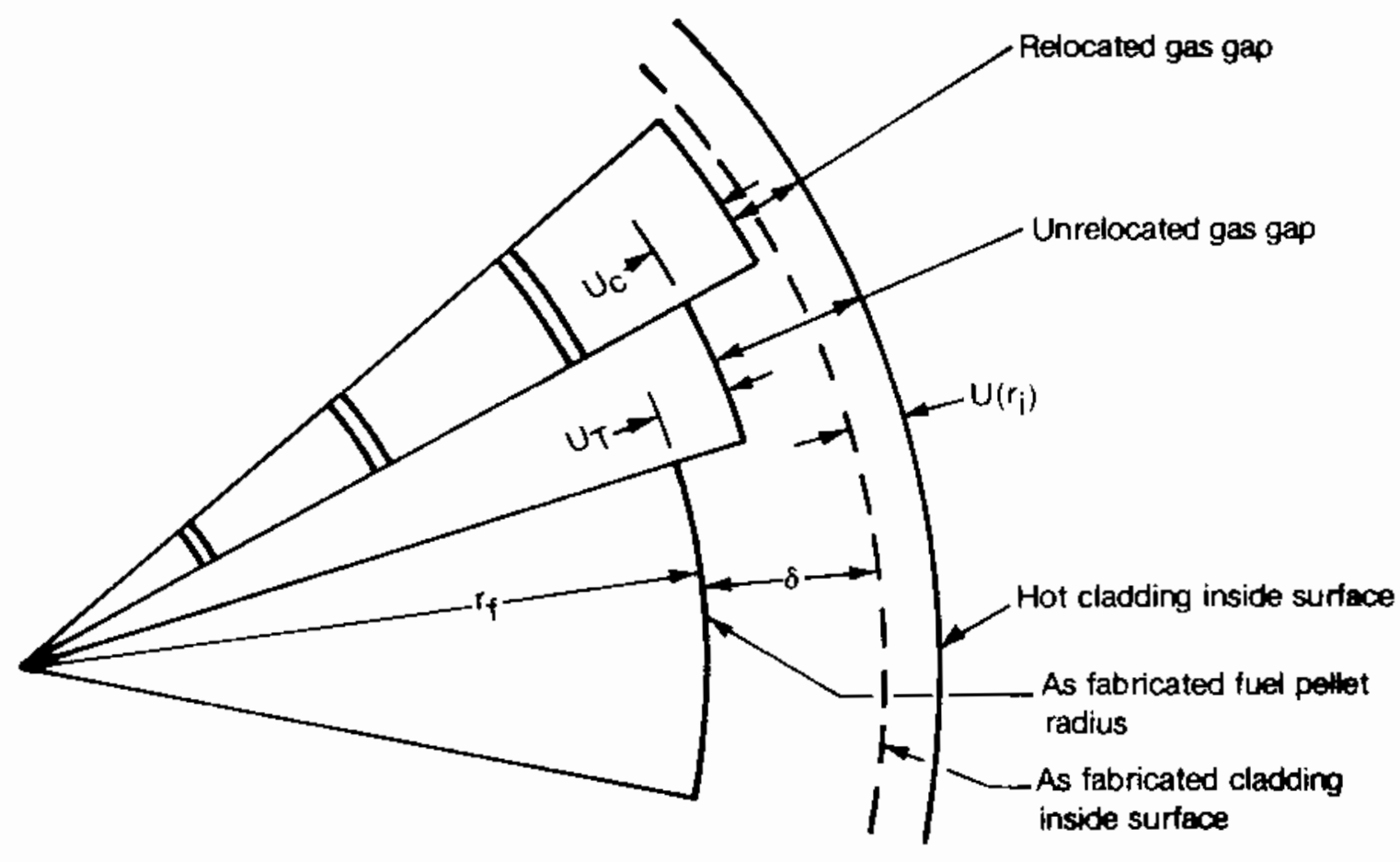

INEL-A-8059

Figure 15. Fuel relocation. 


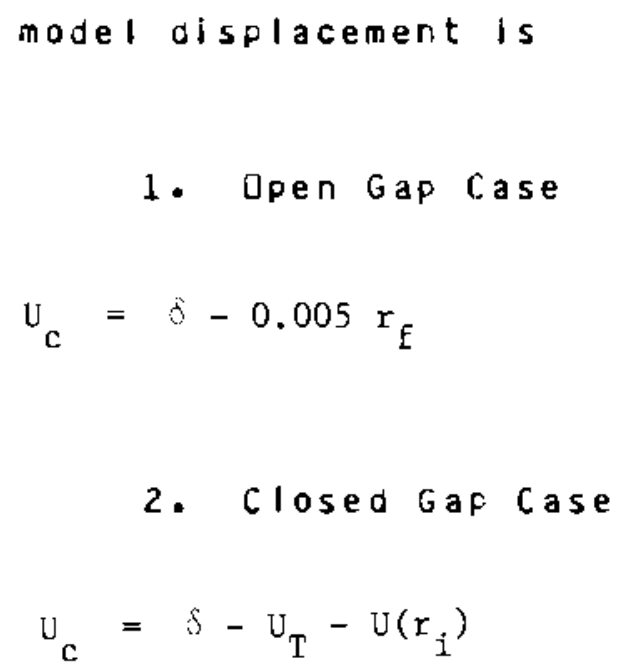

where

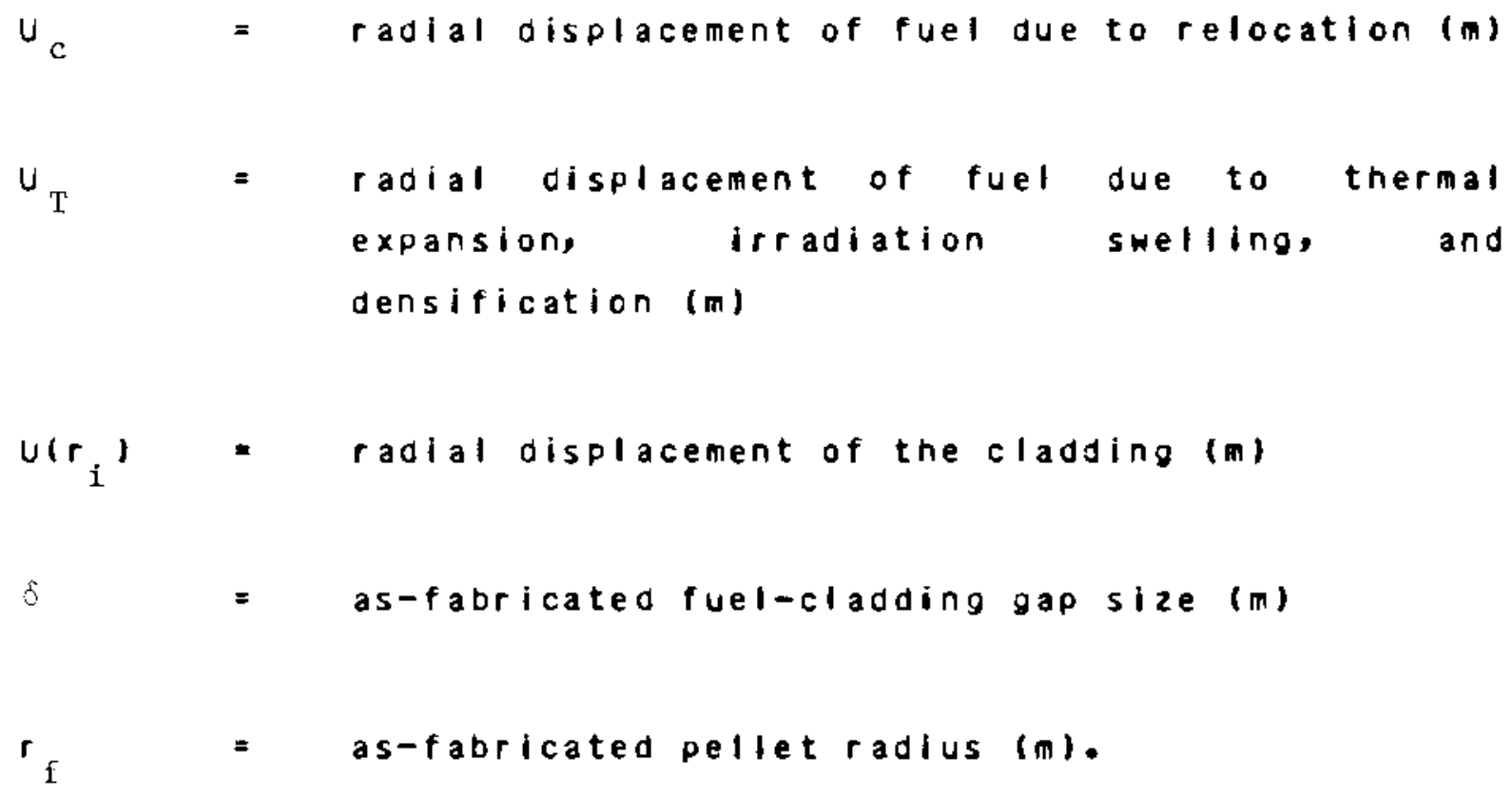

The fuel-cladoing gap size usedin the thermal and internat pressure calculations include the fuel relocation, while the fuelcladding gap size used in the structural calculations does not. The fuel conductivity is modified according to Equation (51) to account 
for the cracks forneo by fuel relocation.

2.4.1.3 Defornable Pellet Deformation Model--The deformable pellet deformation model, fRACAS-II, is used to calculate the fuel rod deforiation when stress effects on fuel deformation become impartant. Tnis model comfutes the stress and strain distributions in toth the fuel and claoding. Elastic and plastio strains in both the fuel and clacoiny are considered. The stresses and strains in the fuel and cladcing are ottained by the transfer matrix appoach. The plastic strains are ottaineo by the Method of Successive suostitutions, which was outlined in section 2.4 .1 .1 .

The method cf cotaining the "pseudo-elastic" solution for fuel rod stresses ano strains required at each plastic strain iteration in the Method of Successive Sutstitutions is described below.

The geometric roosel is a right circular cylinder leither solid or holfow) in a state of generalized plane strain. The applied loads are external pressure, internal pressure lif the cylinder is nolfowl, and dxial force. The cylinger may consist of a single material, or may be a composite cylinger cansisting of two layers of Jifferent materials. An arbitrary radial temperature distribution may te prescribed, and temperature dependent material properties may be usec.

a single layer (homogeneous) cylinder is used to analyze the fuel and the cladding separately before contact occurs. A twolayered (composite) cylinder is used to analyze the fuel and clading after fuel expansion results in firm contact between the fuel ano the claoding. For the composite cylinder case, the stress and strain distributions are permitted to be discontinuous at the 
interface cetween the layers, and the discontinuity in radial displacenient ario axial strain must be deternined. (Ihe Jiscontinuity values are obtained from the displacements which exist in the fuel and clacoing at the instant of fuel-cladoing contact).

ine metnod liseo to solve for the stresses, strains and displacements in the composite cylinder is the transfer matrix approach, as descrited in Reference 24 , modified to consider the state of generalized plane strain. In adoition, the technique nas veen extended to consider displacement discontinuities and both axial and radial cracks in the cylinder.

First, a complete homoceneous cyinder with no discontinuities, out mith variable $E$, $b$, ano $a$ (modulus of elasticity, poisson's ratio, ano coefficient of thermal expansion, respectivelyl is considered. Unty racial variations in temperature, $T$, and material properties are considered. Generalized plane straln deformation is assumeo, so that tor all $r$,

$E_{z}=$ constant

The value of the constartaxial strain, $E_{z}$, is determined from the condition of axial force equilibrium,

$$
\int \sigma_{z} d A=F_{z}
$$

where $F_{z}$ is the resultins axial force. $F_{z}$ is determined from the known internal ano external pressures. 
The governing equations of equilibrium and compatibility in the absence of any dislocations (displacement discontinulties) are given by

$$
\begin{aligned}
& \frac{d \sigma}{d r}+\frac{\sigma_{r}-\sigma_{\theta}}{r}=0 \\
& \frac{d \varepsilon_{\theta}}{d r}+\frac{\varepsilon_{\theta}-\varepsilon_{r}}{r}=0
\end{aligned}
$$

The elastic-plastic stress-strain relations are

$$
\begin{aligned}
& \varepsilon_{r}=\frac{1}{E}\left[\sigma_{r}-v\left(\sigma_{\theta}+\sigma_{z}\right)\right]+\alpha_{r} T+\varepsilon_{r}^{P}+d \varepsilon_{r}^{P} \\
& \varepsilon_{\theta}=\frac{1}{E}\left[\sigma_{0}-v\left(\sigma_{r}+\sigma_{z}\right)\right]+\alpha_{\theta} T+\varepsilon_{\theta}^{P}+d \varepsilon_{\theta}^{P} \\
& \varepsilon_{z}=\frac{1}{E}\left[\sigma_{z}-v\left(\sigma_{r}+\sigma_{\theta}\right)\right]+\alpha_{z} T+\varepsilon_{z}^{P}+d \varepsilon_{z}^{P}
\end{aligned}
$$

Substitution of Equations (123) and (124) into Equation (122) results in

$$
\begin{aligned}
& \frac{\mathrm{d}}{\mathrm{dr}}\left\{\frac{\sigma_{\theta}}{\overline{\mathrm{E}}}-\frac{\nu}{E}\left(\sigma_{\mathrm{r}}+\sigma_{z}\right)+\alpha T+\epsilon_{\theta}^{\mathrm{P}}+\mathrm{d} \varepsilon_{\theta}^{\mathrm{P}}\right\} \\
& +\frac{1+v}{E} \frac{\left(v_{\theta}-{ }^{\sigma} r\right)}{\mathbf{r}}+\frac{\alpha_{\theta} \mathrm{T}-\alpha_{r} \mathrm{~T}}{\mathrm{r}}+\frac{\varepsilon_{0}^{\mathrm{P}}-\varepsilon_{r}^{\mathrm{P}}}{\mathrm{r}} \\
& +\frac{d \varepsilon_{\theta}^{P}-d \varepsilon_{r}^{P}}{r}=0
\end{aligned}
$$

Equations (121) and (126) relate the stresses as they vary across the cylinder. A number of node points are introduced along the 
radius of the cylinger, and the stresses are evaluated only at the nodes. Thus Equations (119), (121), (125) and (126) can be written in finite difference form, and a set of recursion relations.

$$
\left\{\begin{array}{c}
\sigma_{r} \\
\sigma_{\theta} \\
\sigma_{z}
\end{array}\right\} i+1=[L(i)]\left\{\begin{array}{l}
\sigma_{r} \\
\sigma_{\theta} \\
\sigma_{z}
\end{array}\right\}+\{M(i)\}
$$

are obtained. This matrix equation relates the stresses at node i-1 to those at node i. The matrices [L(i)] and [M(i)] depend only on the materials properties, geometry, and plastic strains.

By successive application of Equation (127), a relation between the stresses at any node and the stresses at node 1 the node at the insioe of the cylinoer) can be ootained. This relation takes the form

$$
\{\sigma\}_{i+1}=[a(i)]\{\sigma\}_{i}+\{B(i)\}
$$

where

$\{\sigma\}=\left\{\sigma_{r}, \sigma_{\theta}, \sigma_{z}\right\}$.

The matrices [A(i)] and [B(i)] may be determined from [L(i)] ano [M(i)], as follows:

$$
[A(i)]=[L(i)][A(i-1)]
$$

$\{B(i)\}=[L(i)\}\{B(i-1)\}+\{M(i)\}$ 
for i ireater than 1 , and

$[A(1)]=[L(1)]$

$\mathrm{B}(1) ;=\{\mathrm{M}(1)\}$

for i equal 1 .

by recursion, [A(i)] and [B(i)] across the cylinder wall can be ootained with tne result that

$\{0\}_{N}=[A(N-1)] \quad\{\sigma\}_{1}+\{B(N-1)\}$

At the outside surface, ${ }_{r}=-P_{0}$, where $P_{0}$ is the external (coolant) pressure acting upon the cladoing. Thus, the following concition can be obtained.

$-P_{0}=A_{11}(N-1) o_{r}(1)+A_{12}(N-1) O_{0}(1)+A_{13}(N-1) o_{z}(1)+B_{1}(N-1)$

At the inside surface of the cylinger, one of the following conditions notos:

$\left\{\begin{array}{l}\sigma_{r}(1)=-P_{i}, \quad \text { if } r_{1} \neq 0 \\ \sigma_{r}(1)=\sigma_{0}(1), \text { if } r_{1}=0\end{array}\right.$

(136)

Finally, the condition of axial equilibrium, 
$\int \sigma_{z} d A=\sum_{j=1}^{n-1} \sigma_{z}(j) d A(j)=F_{z}$

must de satisfied. Lsing the recursion matrices, this becones

$$
\begin{aligned}
& \sum_{j=1}^{N-1} \sigma_{z}(j) \mathrm{dA}_{j}=\left[\begin{array}{lll}
0 & 0 & 1
\end{array}\right]\left[[I] \mathrm{d} A_{1}+\left|\mathrm{A}_{1}\right| \mathrm{dA}_{2}+\ldots .+\left|\mathrm{A}_{\mathrm{N}-1}\right| \mathrm{dA} \mathrm{A}_{\mathrm{N}}: \sigma_{1}\right\} \\
& +\left\{0+\left\{\mathrm{B}_{1}\right\} \mathrm{dA}_{2}+\ldots .+\left\{\mathrm{B}_{\mathrm{N}-1}\right\} \mathrm{dA}_{\mathrm{N}}\right\} \\
& \Delta[C]\left\{\sigma_{1}\right\}+\{0\} \text {. }
\end{aligned}
$$

The axial force conoition is the third component of this matrix equation, which can be written as

$$
F_{2}=C_{33_{2} \sigma_{2}(1)+D_{j}}
$$

Equations (135), (136), 1139) are solved simultaneously for the stresses at the inside node $[\sigma]$, after which all other streses and strains can be aetermined from the recursion relations given in Equation (129).

Thus, once the transfer matrites [L(i)] and [M(i)] in Equation (127) are knowr for each annulus in the cylincer, finding the stresses throughot the cylinder becomes a straightormaro procedure.

The advantage of using the transfer matrix approach in solving for fuel rod defornations is that different transfer matrices can be 
used, depending on whether the fuel is cracked axially or radially, or both, and whether or not the cladding and fuel are in contact. The basic solution technique is not changed. The various transfer matrices required are illustrated below.

Homogeneous Cylinder--This section describes the transfer matrices for a homogeneous cylinder in which the radial displacements and $a x+a l$ strains are continuous. The temperature and material properties, nowever, may vary (radially) in an arbitrary manner.

As shown in figure 16 , the cylinder feither hollow or solid) is broken up into N-1 annular regions, with $N$ node points, where $r_{i}$ is the radius to the first node. (For a solid cylinder $r_{i}=0$ ). values of stresses, elastic strains, and plastic strains are founo at each of the node points.

The derivatives are evaluated at the center of each annular region; that is, for the j-th annulus, at

$r=0.5\left(r_{j+1}+r_{j}\right)$

Equations (121) ano (126) are written for the midpoint of each annular region. Thus, for the $j-t h$ annulus,

$\frac{d}{d r}\left(\frac{\sigma_{\theta}}{E}\right)=\left[\frac{\sigma_{\theta(j+1)}}{E(j+1)}-\frac{\sigma_{\theta(j)}}{E(j)}\right] /\left(r_{j+1}-r_{j}\right)$

$(141)$ 
and

$\frac{\sigma_{\theta}}{E}=\left[\frac{\sigma_{\theta}(j+1)}{E(j+1)}+\frac{\sigma_{\theta}(j)}{E(j)}\right] / 2$

(142)

where function values at the miapoint are taken as the average of the function values at the endpoints. Denoting $\Delta r_{j}=r_{j+l}-r_{j}$,

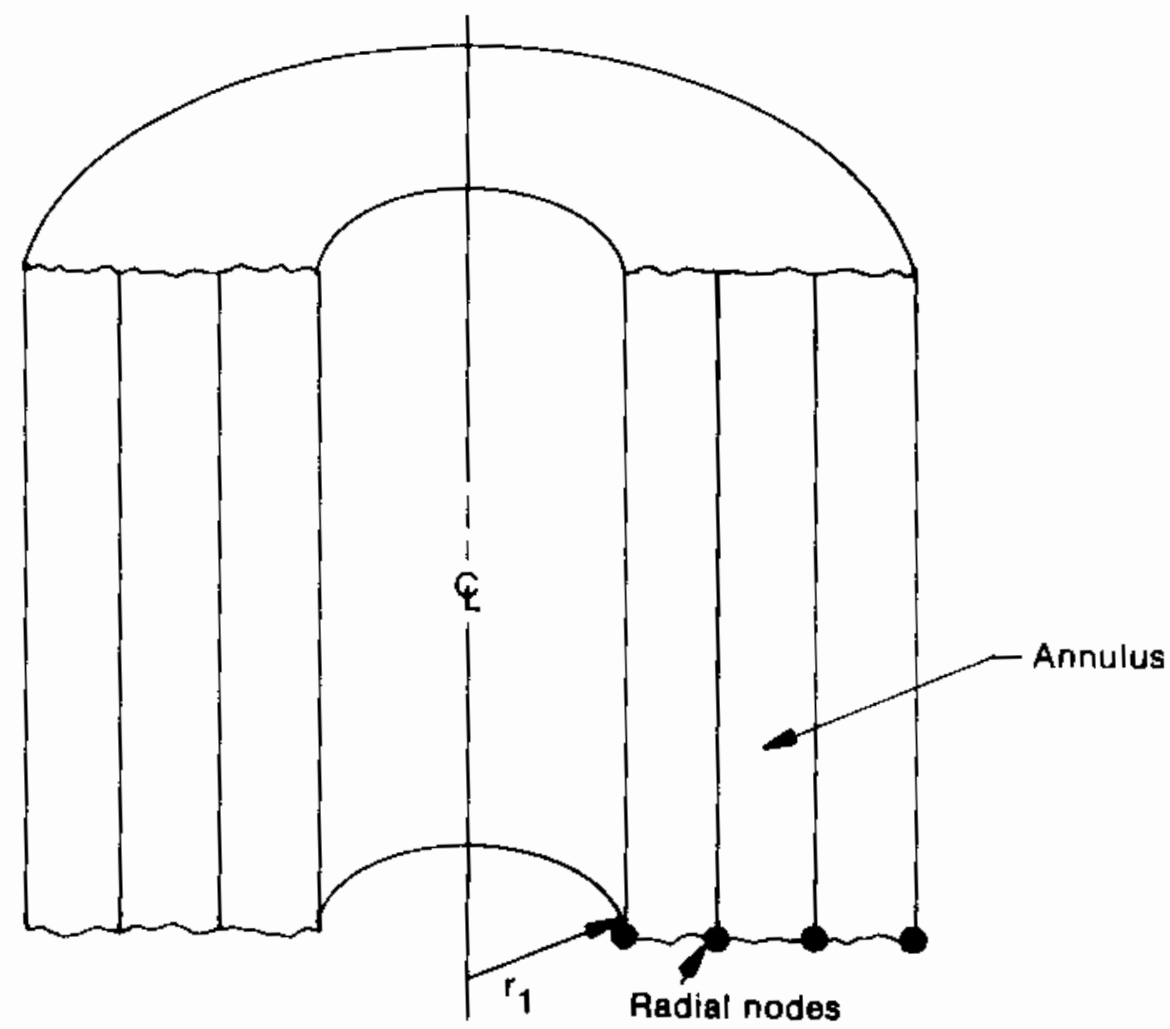

Figure 16. Node and annuli geometry. 
Equations (121), (126), and (119) and (125) become

$$
\begin{aligned}
{\left[\frac{1}{r_{j+1}-r_{j}}+\frac{1}{2 r_{j+1}}\right] \sigma_{r}(j+1)+\left[\frac{-1}{2 r j+1}\right] \sigma_{j}(j+1) } \\
=\left[\frac{1}{r_{J+1}-r_{j}}-\frac{1}{2 r_{j}}\right] \sigma_{r}(j)+\left[\frac{1}{2 r_{j}}\right] \sigma_{e}(j)
\end{aligned}
$$


and

$$
\begin{aligned}
& \left\{\frac{-v(j+1)}{E(j+1) \Delta r(j)}\right\} \sigma_{z}(j+1)+\left\{\frac{v(j)}{E(j) \Delta r(j)}\right\} \sigma_{z}(j) \\
& +\left\{\frac{-v(j+1)}{E(j+1) \Delta r_{j}}-\frac{1+v(j+1)}{2}\right\} \sigma_{r}(j+1) \\
& +\left\{\frac{1}{E(j+1) \Delta r(j)}+\frac{1+v(j+1)}{2 E(j+1) r_{J+1}}\right\} \sigma_{\theta}(j+1) \\
& +\left\{\frac{t v(j)}{E(j) \frac{1+v(j)}{\Delta r(j)}} \quad-\frac{1+v r_{j}}{2 E(j)} \quad \sigma_{r}(j)\right. \\
& +\left\{\frac{-1}{E(j) \Delta r(j)}+\frac{1+v(j)}{2 E(j) r_{j}}\right\} \quad \sigma_{\theta}(j) \\
& +\left\{\left[\alpha_{\theta} T(j+1)+\varepsilon_{\theta}^{p}(j+1)+d e_{\theta}^{p}(j+1)\right]\left(\frac{1}{\Delta r(j)}+\frac{1}{2 r_{j+1}}\right)\right\} \\
& +\left\{\left[x_{\theta} T(j)+\varepsilon_{\theta}^{P}(j)+d \varepsilon_{\theta}^{P}(j)\right] \quad\left[\frac{-1}{\Delta r(j)}+\frac{1}{2 r_{j}}\right]\right\} \\
& +\left\{\frac{-1}{2 r_{j+1}}\left[a_{r} T(j+1)+\varepsilon_{r}^{P}(j+1)+d \varepsilon_{r}^{P}(j+1)\right]\right\} \\
& +\left\{\frac{-1}{2 r_{j}}\left[x_{r} T(j)+e_{r}^{P}(j)+d E_{r}^{P}(j)\right]\right\}=0
\end{aligned}
$$




$$
\begin{aligned}
& \text { and, finaliy } \\
& \overrightarrow{E(j+1)}\left\{\sigma_{z}(j+1)-v(j+1)\left[\sigma_{\tau}(j+1)-\sigma_{\theta}(j+1)\right]\right\} \\
& +\left[a_{z} T+\varepsilon_{z}^{P}+d \varepsilon_{z}^{P}\right]_{j+1} \\
& \frac{1}{E(j)}\left\{\sigma_{z}(j)-v(j)\left[\sigma_{r}(j)+\sigma_{\theta}(j)\right]\right\} \\
& +\left[\alpha_{z} T+c_{z}^{P}+d \varepsilon_{z}^{p}\right]_{j}
\end{aligned}
$$

For the j-th annulus, Equations (143), (144) and (145) may be compactly written as

$$
[E(J)]\left\{\begin{array}{l}
\sigma_{r} \\
\sigma_{\theta} \\
\sigma_{z}
\end{array}\right\}_{j+1}=[F(j)]\left\{\begin{array}{l}
\sigma_{r} \\
\sigma_{\theta} \\
\sigma_{z}
\end{array}\right\}_{j}+\{G(j) j
$$

where [E], [F] ano [G] depend only on the material properties, plastic strains and thermal strains. The axial strains do not occur in the above. Multiplication of this equation by the inverse of [E] results in the equation

$$
\left\{\begin{array}{l}
\sigma_{r} \\
\sigma_{\theta} \\
\sigma_{z}
\end{array}\right\}_{j+1}=[L(j)]\left\{\begin{array}{l}
\sigma_{r} \\
\sigma_{\theta} \\
\sigma_{z}
\end{array}\right\}_{j}+[M(j)\} .
$$

Since neither [E] nor [F] depeno on the plastic strains, the matrices $[E]^{-1}$ and [L] need to be found only once for each load 
step. Hence only [t] [G] need to be recomputed at each step of the iteration in the Method of Successive Substitutions.

Transfer Matrix Across a Surtace of Displacement Discontinuity--one annulus of zero thickness is used as a surface of discontinuity. The radial displacement and axial strain across this discontinuity may be discontinuous. The displacements on each side of the surface are related by

$$
\begin{aligned}
& u_{r}(\dot{k})=u_{r}(k+1)+\Delta u_{r} \\
& \varepsilon_{2}(k)=\varepsilon_{z}(k+1)+\Delta \varepsilon_{2}
\end{aligned}
$$

where $k$ is tre annulus corresponding to the surface of discontinuity. In addition, the radial stress must be continuous, so that

$\sigma_{r}(k)=\sigma_{r}(k+1)$

Then by use of the compatability equations and $\epsilon_{\theta}=u_{x} / r$, Equations $(148)$, (149) and (250) can be written in matrix form as

$$
[P]_{k+1}\{\sigma\}_{k+1}=[P]_{k}\{\sigma\}_{k}+[Q]
$$

The inverse of [P] is easily evaluated explicitly, so that the appropriate transfer relations across the fuel-cladoing interface are 
$\{\sigma\}_{\mathrm{k}+1}=[\mathrm{P}]_{\mathrm{k}+1}^{-1}[\mathrm{P}]_{\mathrm{k}}\{\sigma\}_{\mathrm{k}}+[\mathrm{P}]_{\mathrm{k}+1}^{-1}\{\mathrm{Q}\}$

which is in the form of Equation (120). Similar transfer relations are useo for difterent combinations of axial anc raoial crack patterris.

2.4.2 The PELET/talial Mocel. This section describes the RaDial mocel, which estimates the thermal/mechanical state of the fuel Juring a time step, and the PELET model, which calculates the incremental elastic-plastic cladding oeformation at the eno of the time ster. The interconnection between RADIAL and PELET was indicated in section 2.2 .2 ano is described in detail in appendix $c_{\text {. }}$

2.4.2.1 The kabial Model--The principles oy which hydrostatic stress and free area distribution are derived from in-reactor data were aescribed in section 2.2.2. The free area is that portion of the $(r, \theta)$ area circurscribeo by the cladding which is not occupied by fuel. part cf this area resides in the fuel-clading sap, and the remaincer in the tuel cracks. These same principles and assumptions are applicc in the RADIAL model (mhere current temperatures anu poners are treated as datal to converge on values for the hyarostatic stress ano free area partition for each axial noue for the current time step.

This convergence is accomplished as follows. It is required to bring the quantity (PRG-PRF) below some criterion, where PRG and PRF are the (hyorostatic) stresses in the fuel-cladding gap and fuel cracks, respectively. These two variables can be indefendently calculated ria the Mikic Model given an estimate of total free area, that is, the area which results from the fuel ano cladding temperature and expansion plus an estimate of the current local fuel-cladoing yap size. Choices for the fuel-clading gap size are 
constrained. They must be less than about $3 \sigma$, where $\sigma$ is the standara deviation of the fuel-caldding gap roughness $11 / 2$ peak height). The value of $\sigma$ is fixed in RADIAL as $1 / 5$ of the asfabricated fuel-cladding gap size. (Changes to this gap size occuring from densification, creep, and swelling are taken into account.) The problem is to find some rule for successive variations of fuel-claooing gap size so that convergence is achieved. The method used is sketched in figure 17. The original set of gap size-stress values from the previous time step is varied up and down producing three cases and three values for (PRF-PRG). A parabolic fit through these values gives an estimated fuel-clading gap size where $(P R F-P R G)=0$. The process is then repeated, using the last three values of (PRF-PRG). Convergence is rapid. The effective values for fuel elastic moduli can then be estimated via empirical correlations which will now be described.

The values deduced for the effective radial and axial modui from in-reactor data have been found to correlate particularly weII witn certain parameters: the radial modulus correlates well with estimated local crack area and the deduced effective axial modulus correlates well with the calculated local total free area. The specific rods examined are listed in Table 2. Note that they span the range (in terms of cold free area) of U.S. power reactor fuel rod designs. This is emphasized in figure 18. Plots of deduced elastic moduli versus void area are shomn in figures 19 and 20 . The respective void areas are expressed as percent of total area within tne cladding. Note that both moduli exhibit distinct separation accoroing to cold free volume, in addition to having strong dependence on the change in void area with increasing power. This segregation is net a measurement anomaly; replicate rods from various tests produced highly similar results. In addition, rod 6 in test IFA-513 and Rod 1 of test IFA-432, although identical in design, are different in fill gas composition and hence, in power- 
VARIATION 1

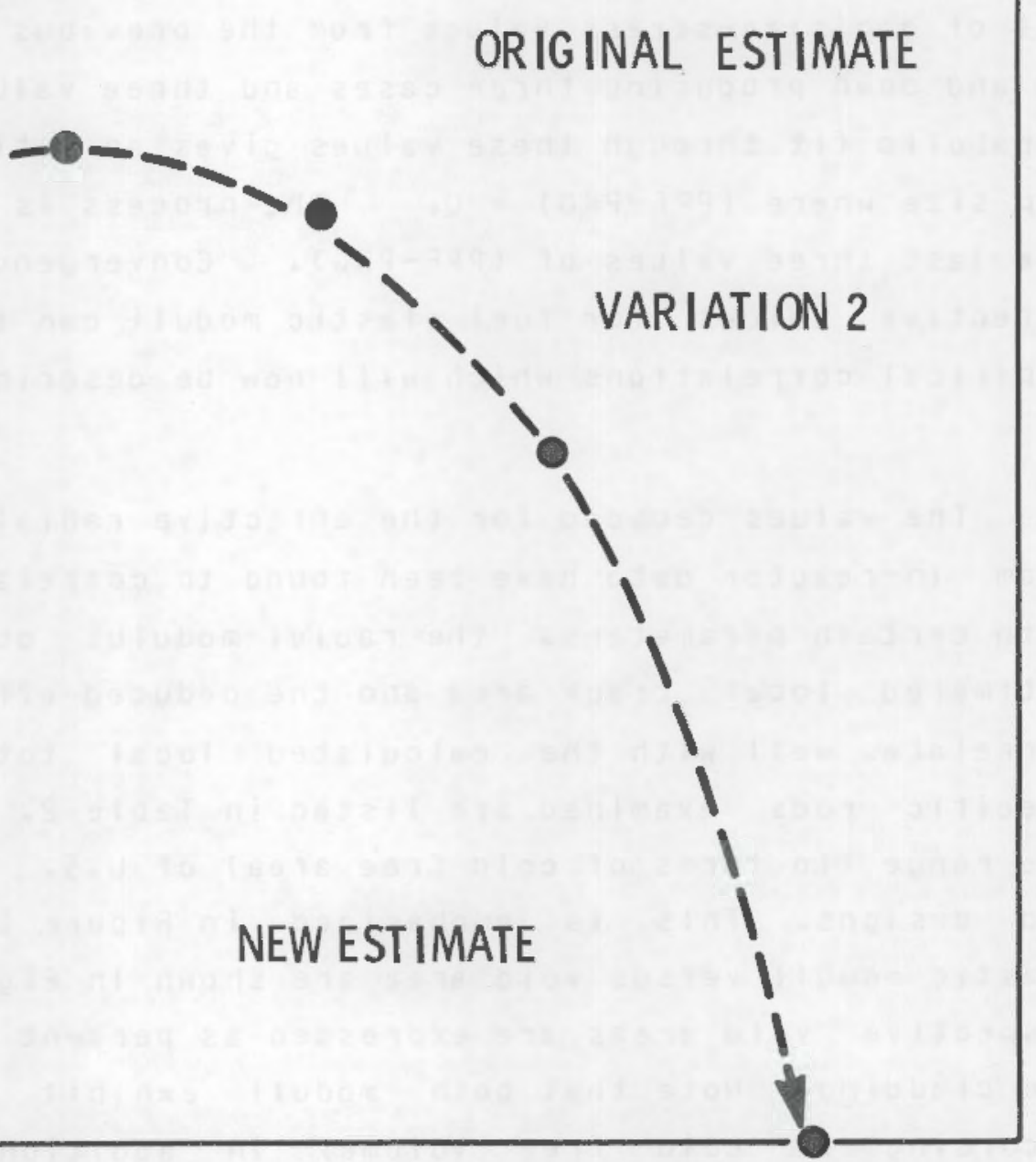

Gap Size

Figure 17. Convergence method for determining gap size and interfactal (hydrostatic) pressure. 
TABLE 2. RODS EXAMINED FOR FUEL ELASTIC MODULUS CORRELATIONS

(ALL 10\% ENRICHED)

\begin{tabular}{|c|c|c|c|c|}
\hline $\begin{array}{l}\text { Halden Reactor } \\
\text { Assembly Number }\end{array}$ & $\begin{array}{l}\text { Rod } \\
\text { Number }\end{array}$ & $\begin{array}{c}\text { Fuel PeI I et } \\
\text { Diameter }(\pi \mathrm{m})\end{array}$ & $\begin{array}{c}\text { Diametral } \\
\text { Gap }(m)\end{array}$ & $\begin{array}{c}\text { Fill Gas } \\
\text { composition }\end{array}$ \\
\hline 432 & 1 & 10.68 & 230 & $\mathrm{He}$ \\
\hline 432 & 2 & 10.52 & 380 & He \\
\hline 432 & 3 & 10.83 & 75 & $\mathrm{He}$ \\
\hline 513 & 6 & 10.68 & 230 & $H \in, x \in(77 \pi, 23 \pi)$ \\
\hline
\end{tabular}

temperature relationship. Their correspondence on both plots attests to both the adequacy of the correlating parameters and the reality of segregation according to initial void area.

These facts provide a basis for correlating effective moduli with current free void and its partition. The elements of these correlations (identical in logic for both moduli) are best shown schematically. In figures 21 and 22 limit lines are shown for the radial and axial elastic moduli, respectively. These represent upper and lower (zero- and high-power) limits on the moduli as a function of crack and total void area, respectively.

A hypothetical path for a particular fuel rod during ascension to power is traced on each plot given in Figures 21 and 22 . As power and temperature increase, the void fraction decreases. At 


\section{RANGE OF U.S. POWER}

REACTOR DESIGNS
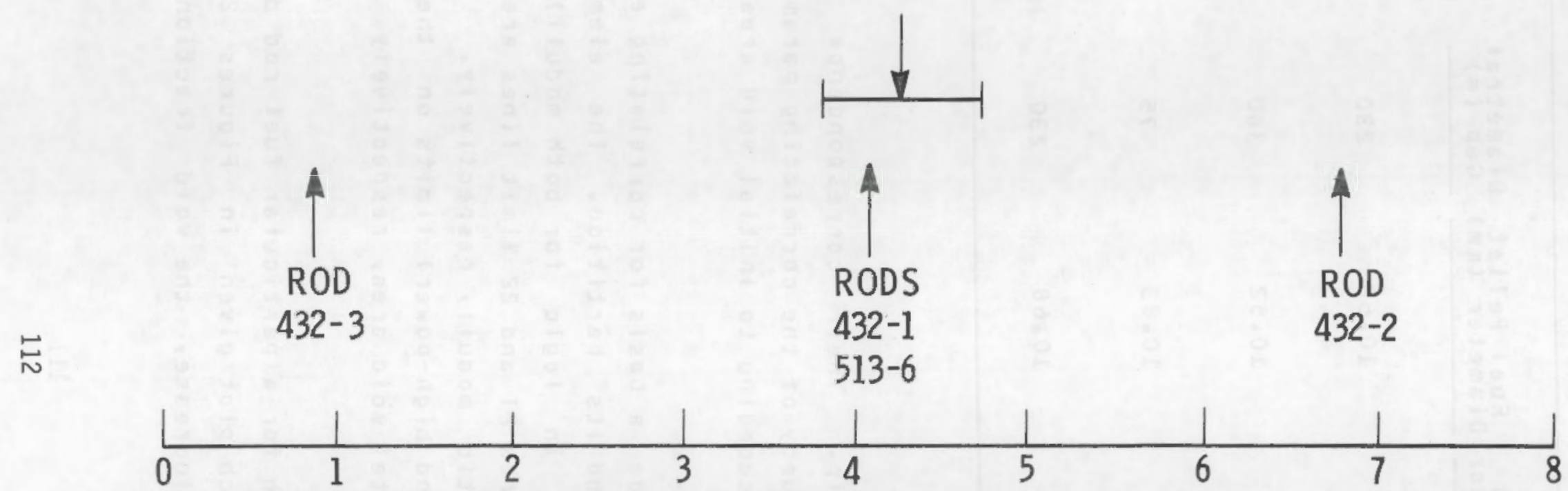

Cold Free Area, \% of Enscribed Area 


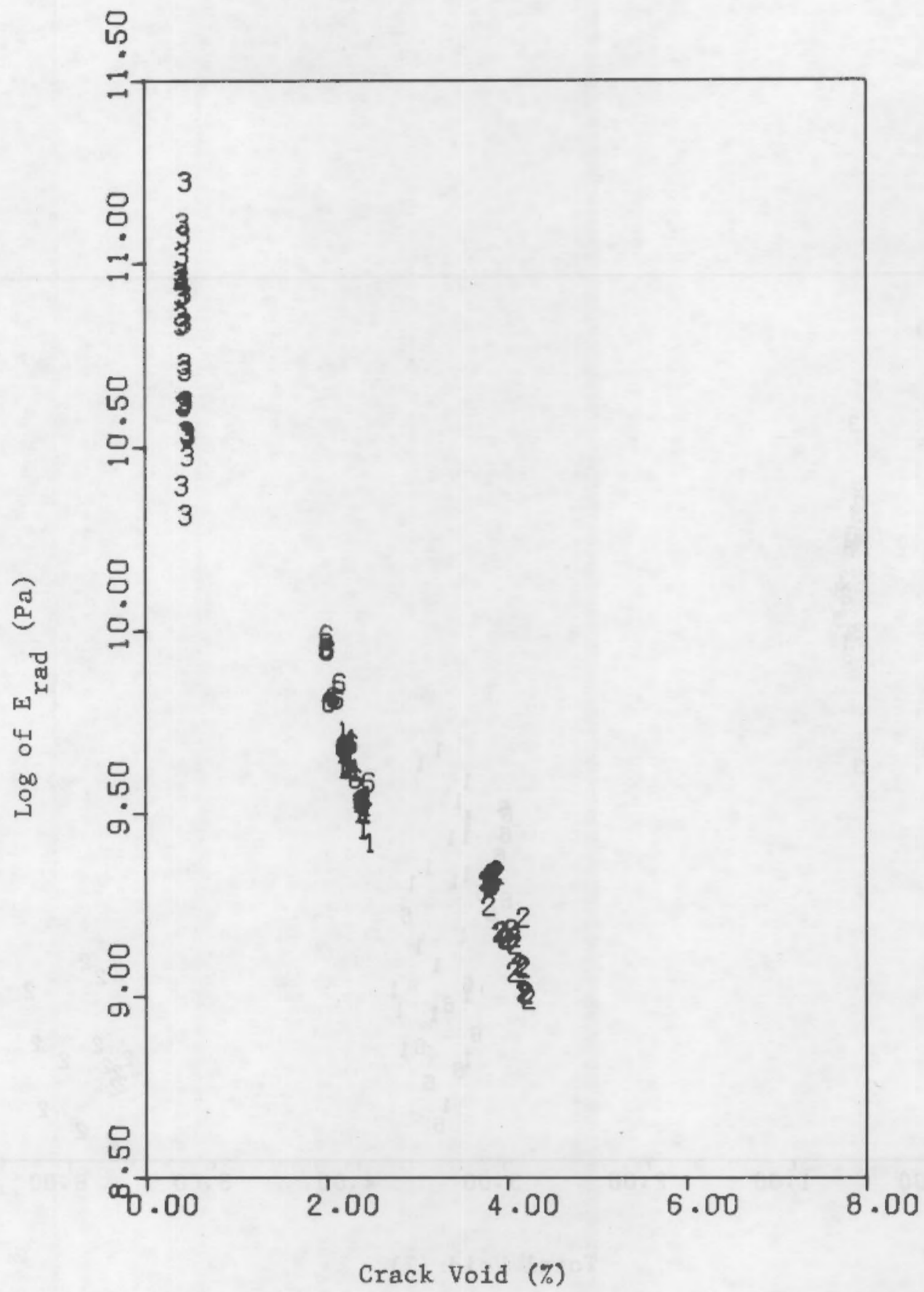

Figure 19. Radial elastic modulus as a function of fuel available void (data points identified by rod number). 


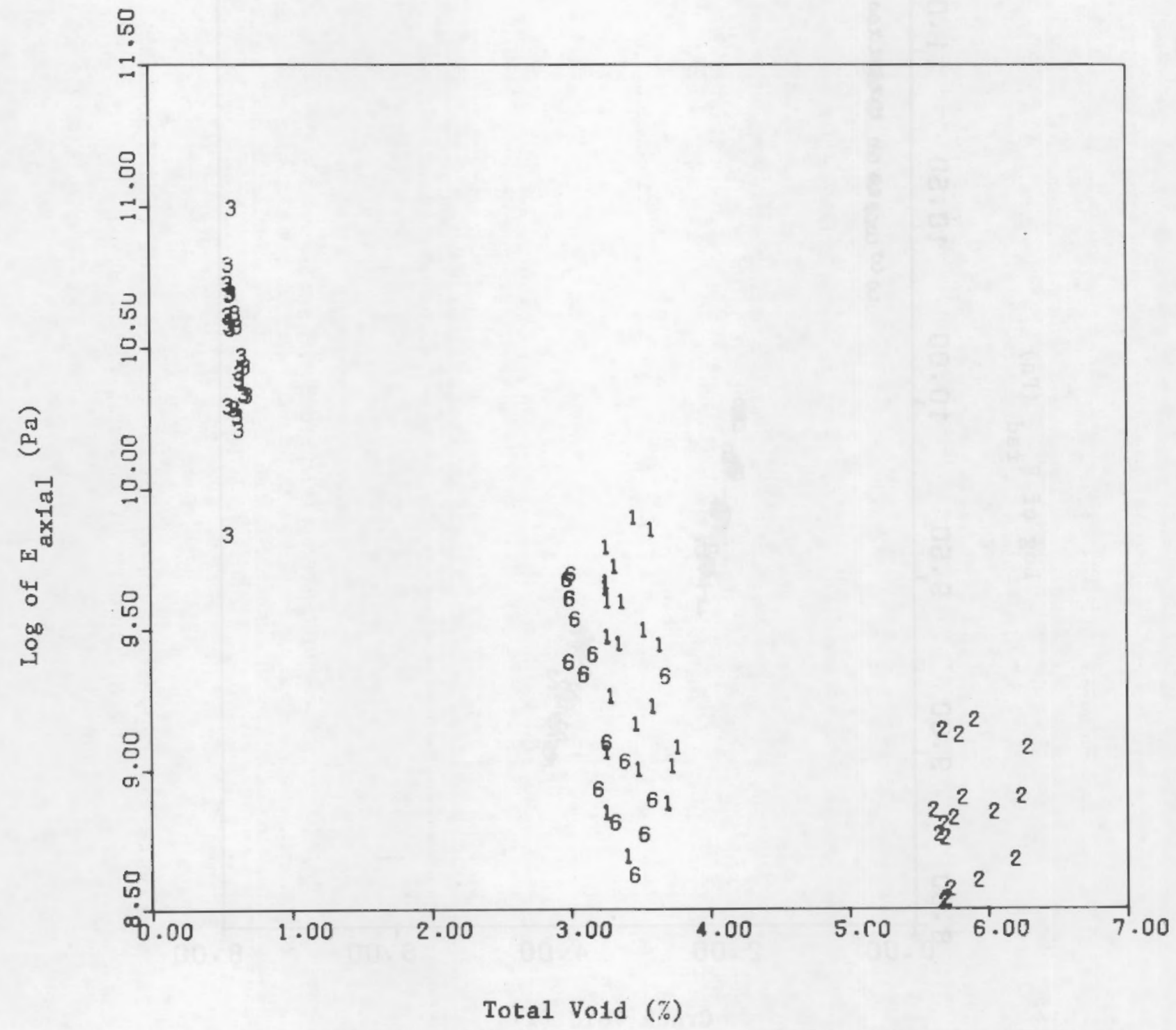

Figure 20. Axial elastic modulus as a function of total available void (data points Identified by rod number). 


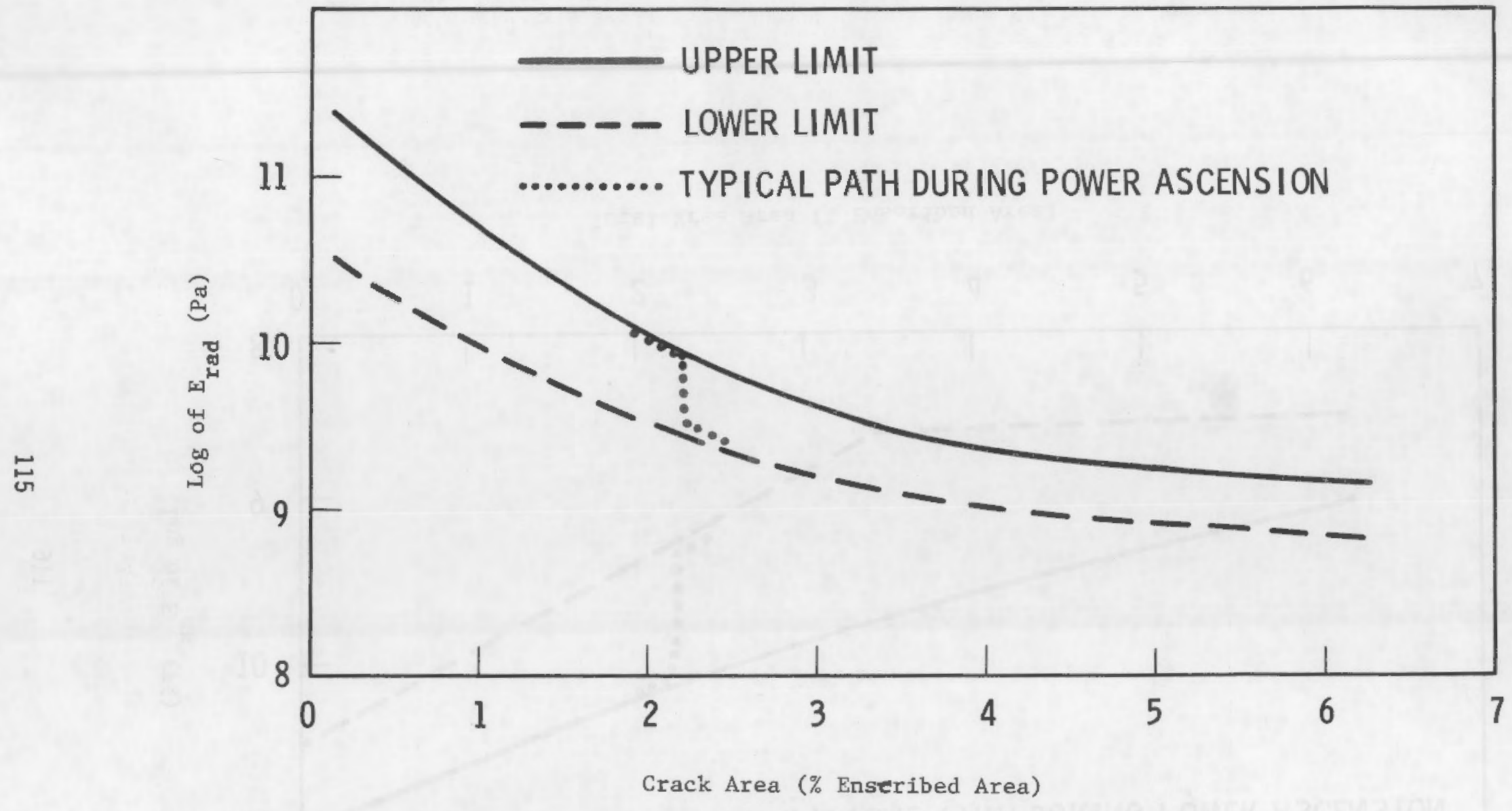

Figure 21. Upper and lower limits of the fuel radial modulus as a function of current crack area. 


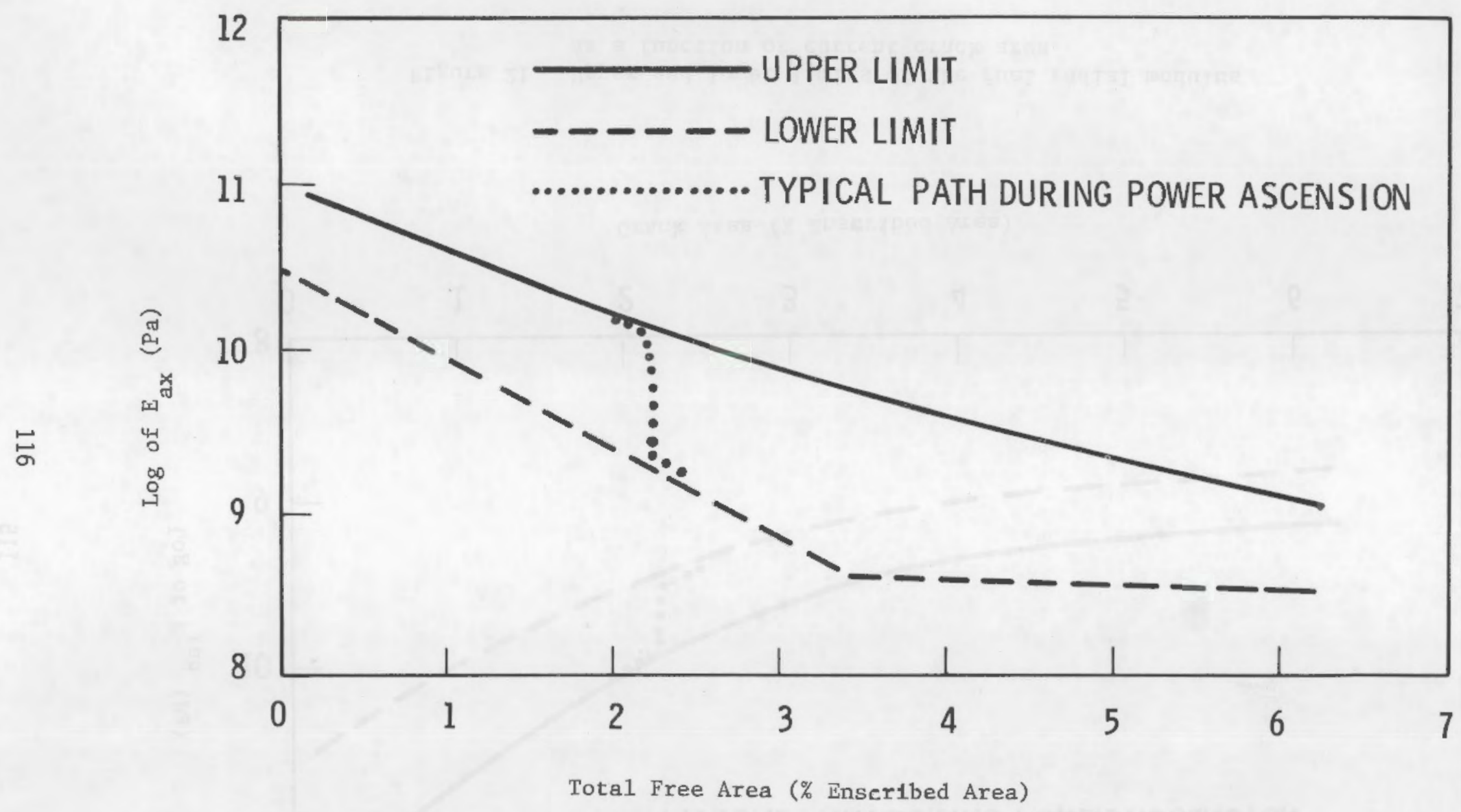

Figure 22. Upper and lower limits for the fuel axial modulus as a function of current total free area. 
first, the modulus is allowed to respond to decreasing void fraction by traveling only along the lower limit curve. However, with a continually decreasing void, the modulus is alloned to travel toward the upper limit curve and is constrained to follow that curve having intersecteo it. The criteria governing departure from the lower limit curve and travel toward the upper limit curve is discussed below.

From cladding elongation data, a distinct change in slope for elongation versus power plots was observed at about the point where 15\% of the total (cold) free void is estimated to be consumed by fuel thermal expansion. Accordingly, this is established as the point of departure from the lower limit curve. Similarly, it has been observed that the slopes of the cladoing elongation versus power curves tend to increase only until fuel volume average temperature exceeds the temperature at the departure point by about $280 \mathrm{~K}$. Accordingly, the ratio $\frac{\bar{T}-\bar{T}_{D}}{280}$ defines the fraction of the total distance between the two curves which the rod will attain. In the above, $\bar{T}$ is the current volume average fuel temperature and $\bar{T}_{c}$ is fuel average temperature at the time of departure from the lower-limit curve. Ihese criteria apply to both moduil.

2.4.2.2 The PELET Package for Mechanical Analysis--PELET is a modification of the chained $r$ adial and axial finite element models used in the GAPCON-3 5 fuel performance code. The axial and raoial models are "chained" in the sense that the axial stresses from the axial model constrain the radial calculations, which apply to representative sices of the fuel at each axial node. This section reviews both the original calculational procedure and the nature of the modifications made to it. Inis review is followed by a detalled 
discussion of the basic models and solution procedures. Finally, the methods by which cladding creep and plasticity are handed in PELET are described.

Modifications to the GAPCON-3 Procedure--The GAPCON-3 procedure for finding the incremental elastic deformation and stress in the cladoing is:

1. Representative thin slices of fuel rod lat midpoints of axial regions) are modeled by a series of axisymmetric triangular elements (see Figures 23 and 24 ). Incremental thermal strain on these elements is treated as initial strain, ano translated into loads. These are combined with incremental pressure loads to find incremental stress in the elements.

2. The estimate of incremental fuel-cladding interfacial pressure (for regions in contact) is translated to radial loads which are applied to a comprehensive axial model of the total fuel roo (see figure 25). Only axial stresses and strains are retained from this model.

3. The axial stresses within each axial region are translated to axial loads for a final pass through the radial models.

This chained radial-axial-radial procedure is modified in the PELET model in the following ways:

1. All axial regions are assumed to always be in contact. Therefore, radial model calculations are 


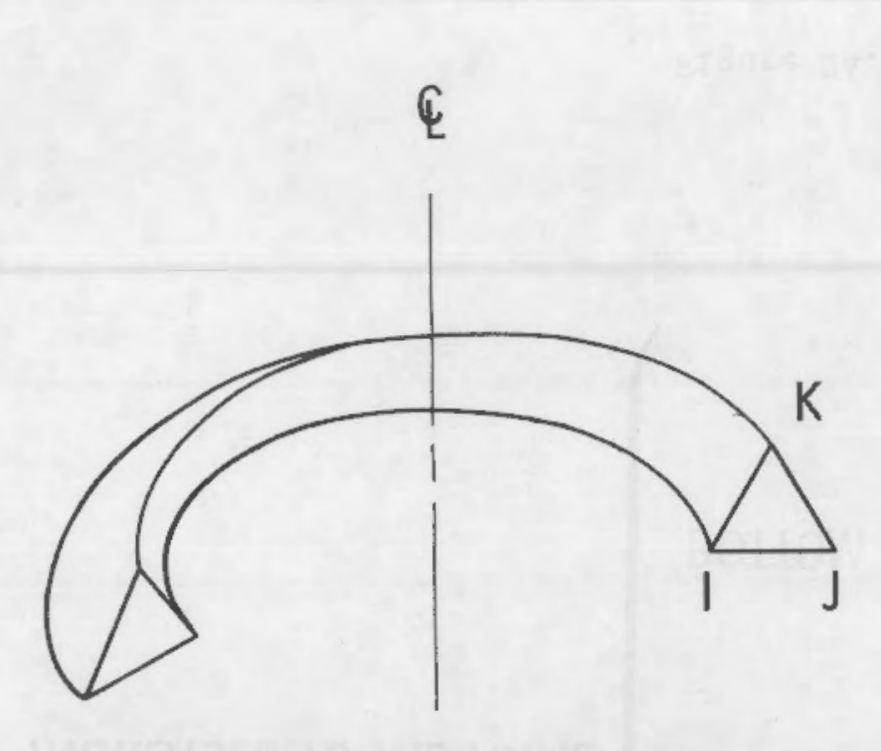

AXISYMMETRIC RING FINITE ELEMENT

$$
[K]\{a\}=\left\{f_{a}\right\}+\left\{f_{a}\right\} \cdots\left\{f_{i}\right\}
$$

\section{BASIC EQUATION}

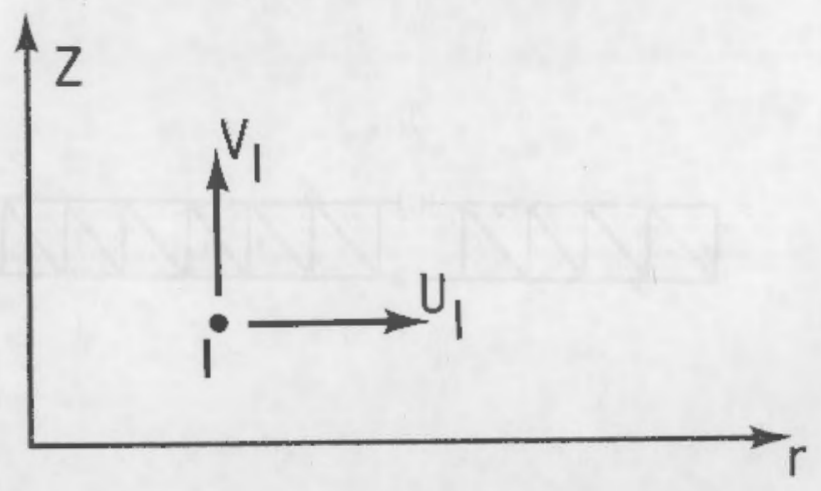

\section{WHERE:}

$\stackrel{\rightleftarrows}{\omega}$ 


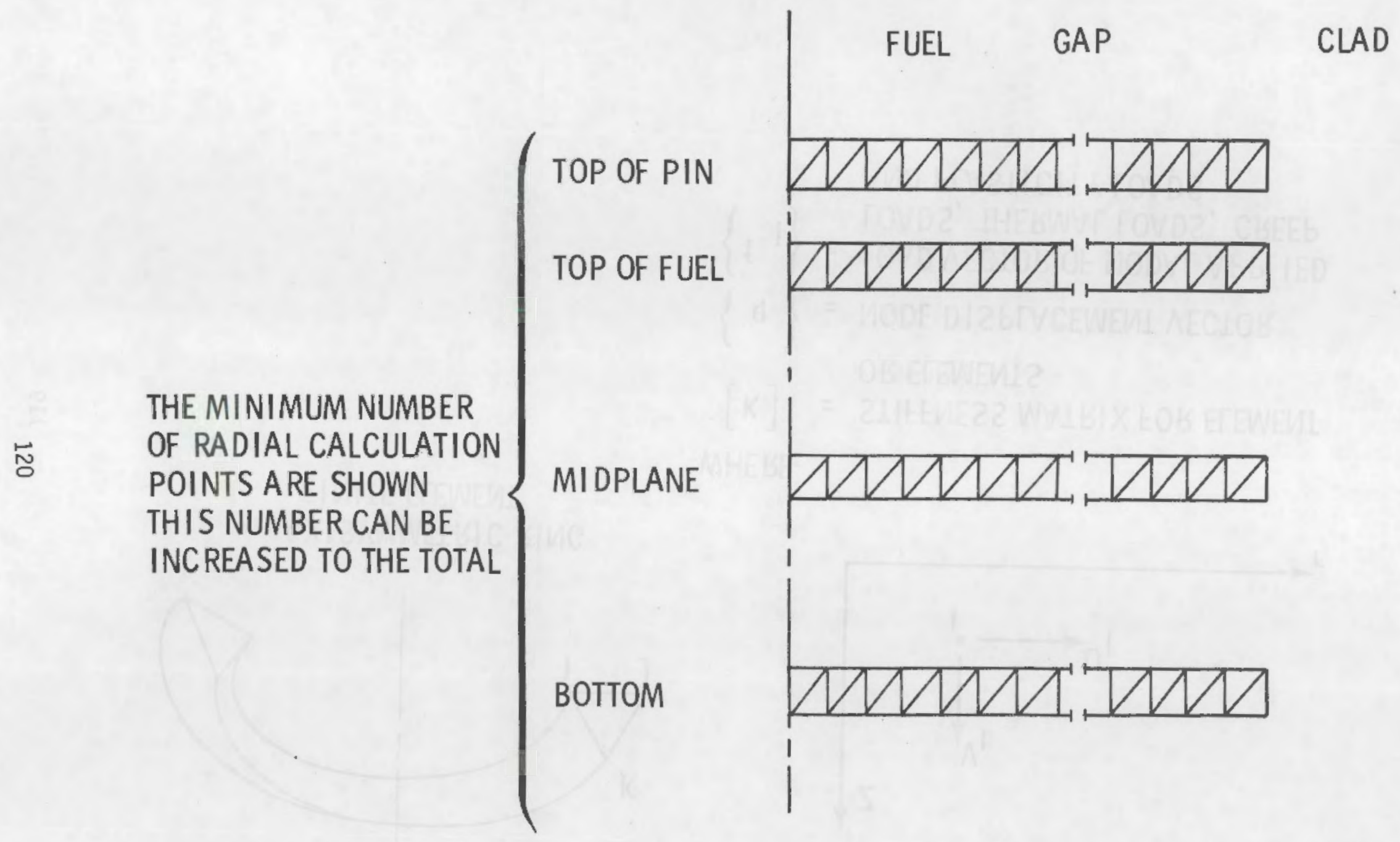

Figure 24. Radial calculation model. 


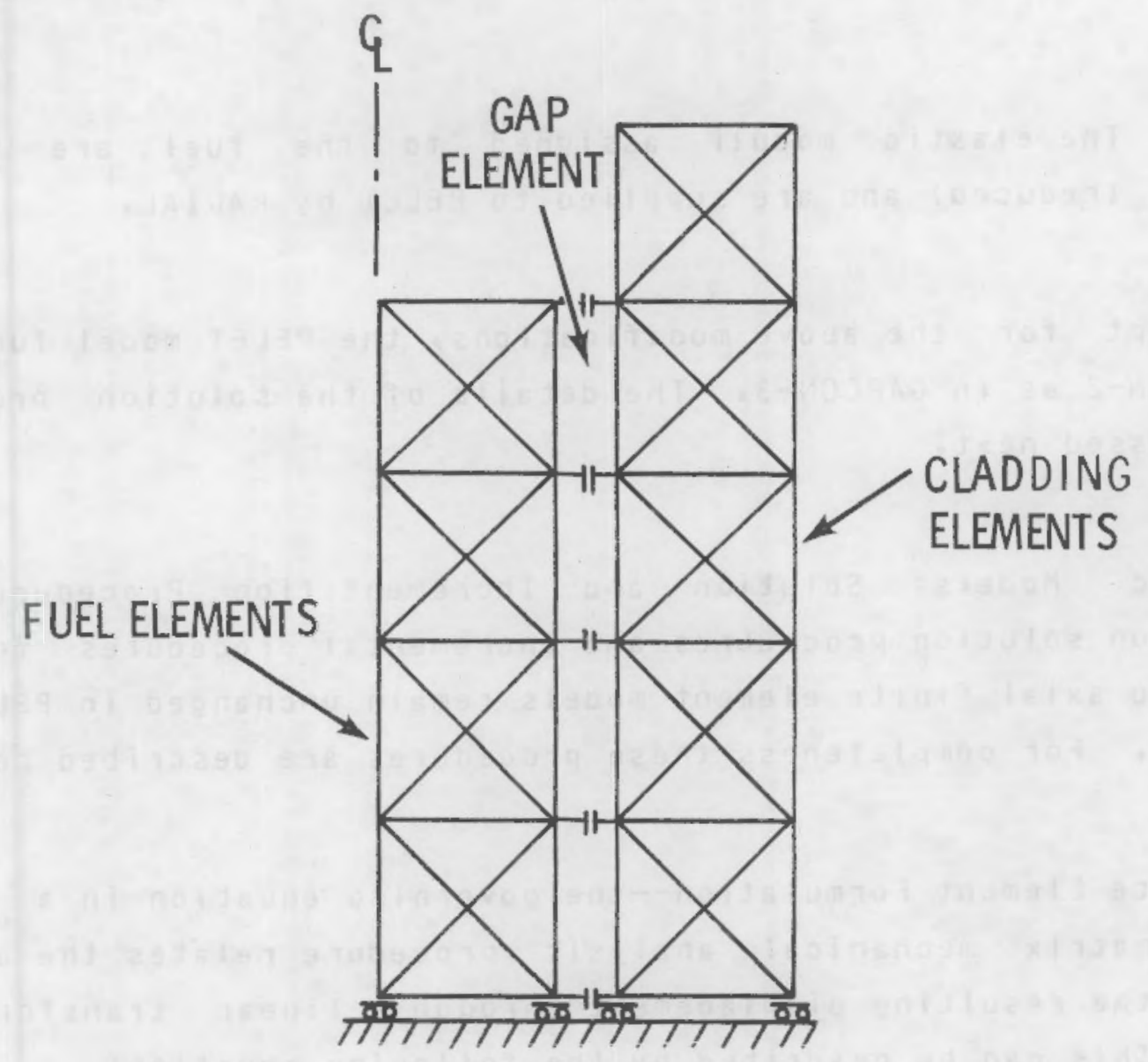

Figure 25. The axial mechanical analysis model using a quadrilateral element composed of four triangular elements. Cladding is accomplished by closure of the gap elements.

performed at all regions.

2. Next, the RADIAL model provides the estimate of incremental interfacial pressure; therefore, the first pass through the radial model has been eliminated. 
3. The elastic moduli assigned to the fuel are altered (reduced) ano are supplied to PELET by RADIAL.

Except for the above modifications, the PELET model functions in FRAFCGN-2 as in GAPCCN-3. The details of the solution procedure are discussed next.

Basic Models: Solution and Incrementation Procedures--The formulation soluticn proceoures and incremental procedures for the radial and axial finite element models remain unchanged in PELET for FRAPCON-2. For completeness these procedures are described below.

Finite Element formulation--the governing equation in a finite element matrix mechanical analysis procedure relates the applied loads to the resulting oisplacement through a linear transformation matrix. This can te oescribed by the following equation:

$\left.[\mathrm{K}]_{[\mathrm{q}}\right]_{i}=[\mathrm{f}]_{\text {external }}+[\mathrm{f}]_{\text {creep }}+[f]_{\text {thermal }}$

$$
+\left[\mathrm{f}_{\text {plastic }}=[\mathrm{f}]_{i}\right.
$$

where

[f] plastic = incremental load vector due to plastic loads

[f] external = incremental load vector due to external loads

$[f]_{\text {creep }}=$ incremental load vector due to creep loads 
$[f]_{\text {thermal }}=$ incremental load vector due to thermal loads

[a] = nodal oisplacement vector for a given incremental load vector

[K] = stiffness matrix or tranformation matrix relating applied loads and displacements.

The fuel-cladoing system experiences strains resulting from displacements due to thermal expansion, internal and external pressure, and fuel-cladding interaction. Further displacement strains are incurred though creep and plasticity. In PELET these sources of strain are expresseo as line loads [f] $i$ at the element boundaries.

Changes in the element stresses and strains resulting from changes in the increncental load vectors [f] $]_{i}$ are found by:

1. Translating thermal expansion and external load changes to incrmental nodal load vectors [f] ${ }_{i}$

2. Solving Equation (153) for the entire model to get the nodal disflacements.

3. Translating the nodal displacements of each element to element strain.

4. Subtracting the initial strain. (see the following sections). 
5. Calculating stress from strain.

6. Correcting for creep and plastic strains by translating these to nodal loads and repeating steps 1 through 5.

The stiffness matrix for the entire model is assembled from the matrices for the individual elements, subject to compatibility of nodal displacements ard to boundary conditions. Each element matrix is a 6 by 6 symmetric matrix, reflecting $51 x$ degrees of freedom (radial and axial movement of the three corner node points). The matrices for all elements are identical algebraically, since all elements are identical structurally. The numerical values of the $k$ from the different matrices are different for the following reasons.

1. The elements differ geometrically. (Nodes and internal degrees of freedom are always numbered counter-clockwise beginning at the lower left corner. For some elements $r_{1}=$ $r_{3}$ whereas for others $r_{2}=r_{3}$, etc.l.

2. The material properties will be evaluated at the element temperature which differs from element to element.

3. The dimensions of the elements will vary as deformation proceeds.

The single element shown in Figure 23 ioentifies the three triangular nodal points IJK. The figure indicates that for each node there are two displacements, $u$ and $v$, corresponding to the radial and axial directions for a total of six displacements for a given element. The element stiffness matrix [K] is then a 6 by 6 
matrix relating the six unknown displacements with six loads applied at the element nodes.

The derivation of Equation (253) is based on the minimization of the potential, or strain energy, in a continuum which can be specializeo to a separate element. The strain energy can be written as shown in Equations (154), (155), and (156) using the concept of virtual strains ana displacements and relating the strain energy of the body to the work done by body loads and surface loads:

$$
\begin{aligned}
& \Pi=\int_{\text {vol }}^{\mathrm{dU}}-\int \begin{array}{l}
\mathrm{xdV} \\
\text { vol }
\end{array}-\int \begin{array}{l}
\mathrm{Tds} \\
\text { surface }
\end{array} \\
& d U=\frac{1}{2}\{\varepsilon\}^{T}\{\sigma\} d V=\frac{1}{2}\{\varepsilon\}^{T}[C]\left[\left(\{\varepsilon\}-\left\{\varepsilon_{0}\right\}\right)\right] d V
\end{aligned}
$$

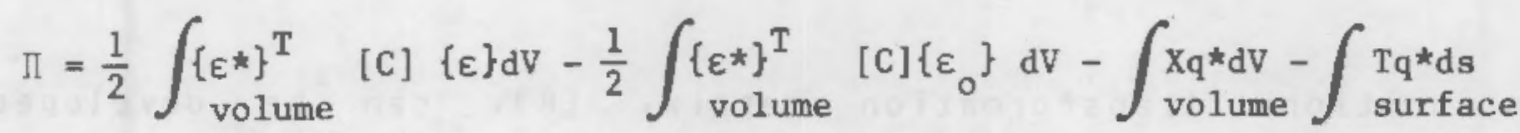

where

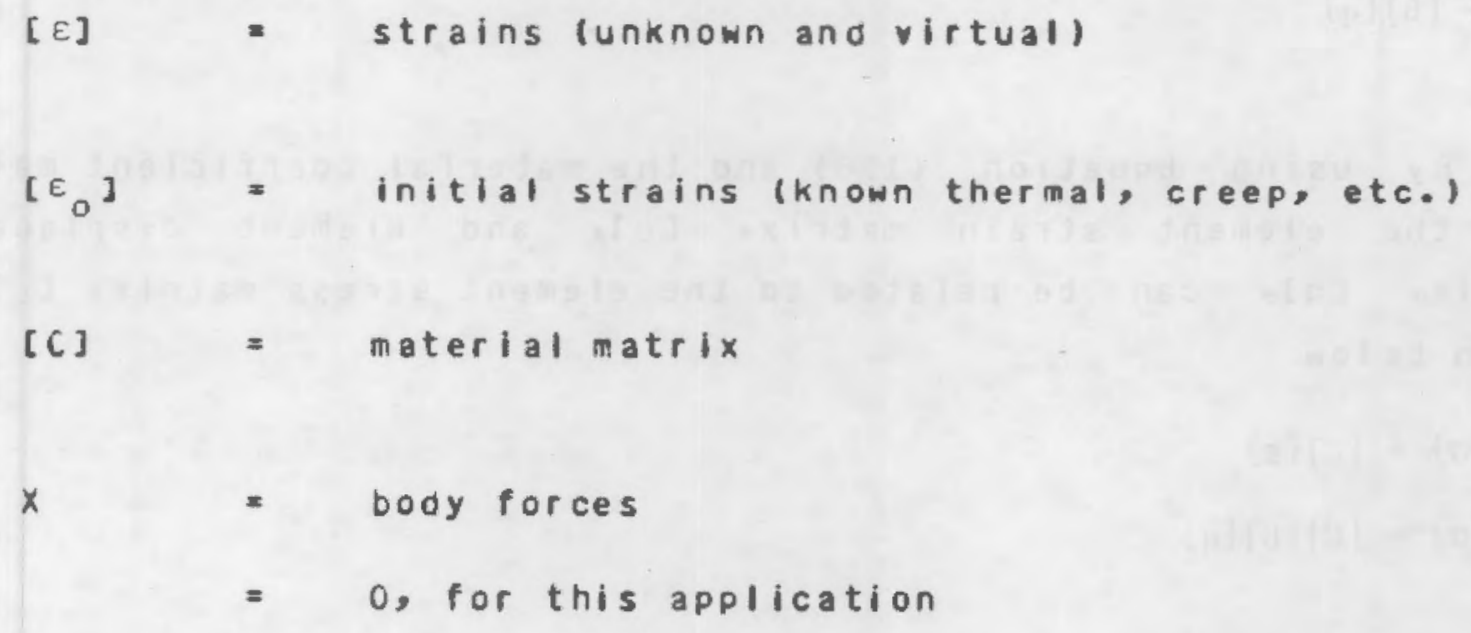




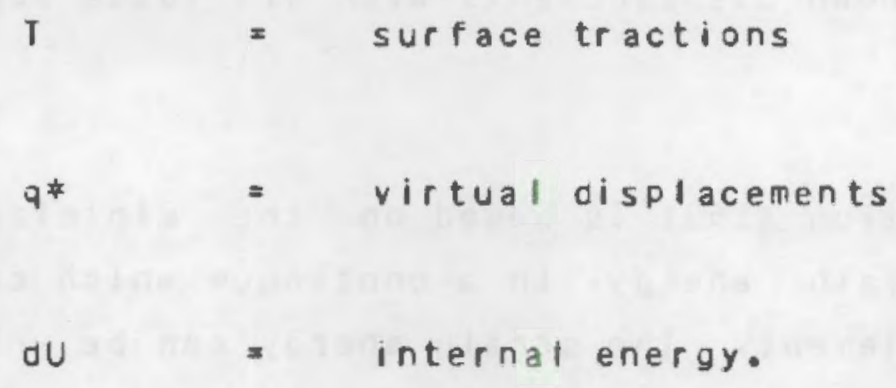

The component parts of Equation (156) can be rewritten in terms of matrix notation for an element where internal stresses and strains are described in terms of element nodal values. Equation (157) defines a transformation matrix, [N], for an interpolation function which relates the displacements of the element nodal points [q] with the displacements internal to the element [u].

$$
\{u\}=[N]\{q\}
$$

An additional transformation matrix, [B], can te developed which relates the element nodal point displacements, [q], with the element strains, [E], as defined in Equation (158).

$$
\{\varepsilon\}+[B]\{q\}
$$

By using Equation (158) and the material coefficient matrix, $[C]$, the element strain matrix, $[E]$, and element displacement matrix, [q], can be related to the element stress matrix, [o], as shown telow

$$
\text { or } \begin{aligned}
\{\sigma\} & =[C]\{\varepsilon\} \\
\{\sigma\} & =[C][B]\{q\}
\end{aligned}
$$


The expression for cescribing the potential energy [Equation $(154) 1$ can be rewritten by direct substitution of Equations (157), $(158)$, and $(159)$ as

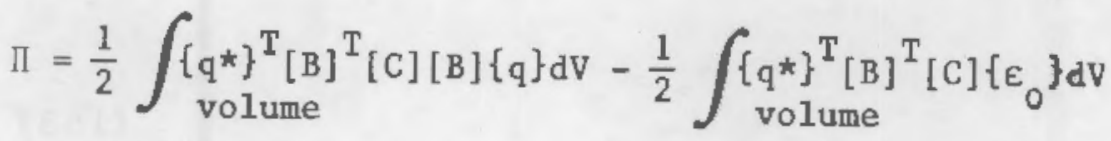

$$
\begin{aligned}
& -\underset{\text { volume }}{\left.\left\{q^{*}\right\}^{T}[N]\right]^{T}\{x\} d V-\int q_{\text {surface }}\{\}^{T}[N]{ }^{T}\{d\} d s}
\end{aligned}
$$

$(160)$

Using the variational principle for finaing the minimum potential energy shown in Equation (154) and differentiating with respect to the virtual oisplacements, the expression can be rewritten in terms of the functional $\Pi$, the strain energy $U$ and the external work W as shown in Equation (161).

$$
\Delta \Pi=\Delta \mathrm{U}+\Delta \mathrm{W}=0
$$

Extracting the virtual displacement vector [q] from Equation (160) anc performing the first variations indicated in Equation (161) with respect to the virtual displacements, Equation (162) is obtained.

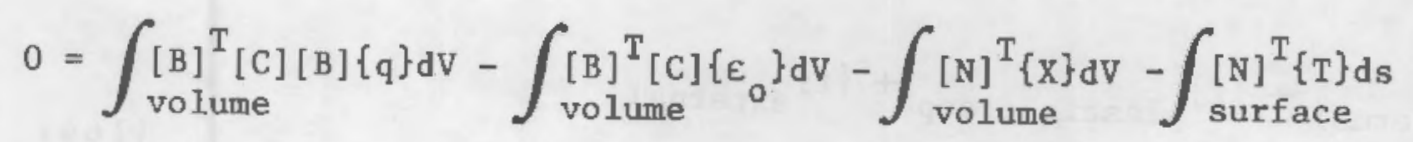

Equation (162) can be rewritten for this specific application by neglecting the body forces to obtain 


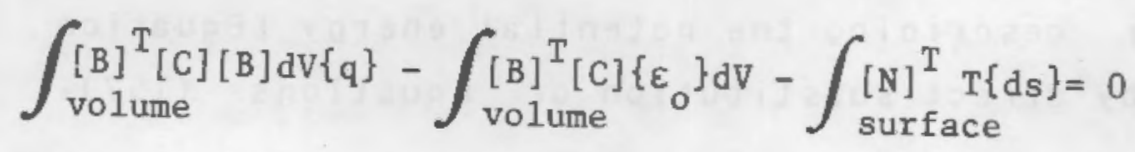

or

$[K]\{q\}=\left\{f_{b}\right\}+\left\{f_{T}\right\}$

where

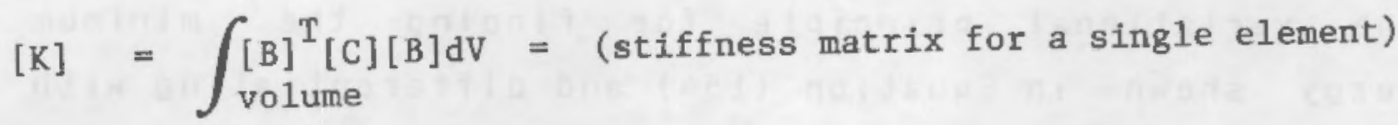

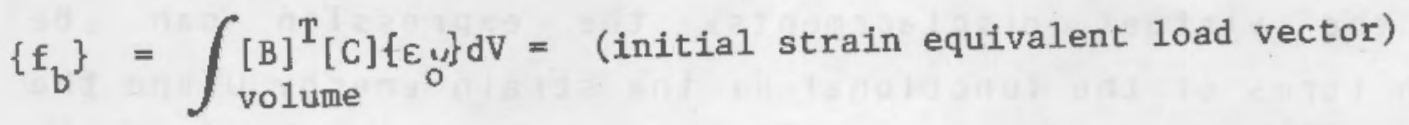

$\left\{\mathrm{f}_{\mathrm{T}}\right\}=\int_{\text {surface }}^{[\mathrm{N}]^{\mathrm{T}}\{\mathrm{T}\} \mathrm{ds}}=\begin{aligned} & \text { (external applied surface forces translated } \\ & \text { into nodal loads) }\end{aligned}$

The matrices [B], [C] and [N] have yet to be oefined for an axisymetric constant strain triangular element.

For the case of interest, the initial strain term [ $\left.\varepsilon_{0}\right]$ takes the form of thermal, plasticity, and creep strains. Equation (153) can then be rewritten as

$[K]\{q\}=\{f\}_{\text {thermal }}+\{f\}_{\text {plastic creep }}+\{f\}$ external

which is identical to Equation (153).

The actual application of this procedure to a given problem 
requires the selection of an element configuration (triangle, square, or rectangle) and the shape function [N] for describing the relationship between nodal point displacements [q] and oisplacements internal to the element [U].

In the case of FRAPCON-2, the element shape is a triangular ring. The triangular element is the least complicated of any of the special elements. The operation defined in Equation (163) indicates that the element formulation requires a volume integral to be performed. The axisymmetric triangular ring element allows integration as shown below.

$[K]=2 \pi \iint[B]^{T}[C][B] r d r d z$

An approximation to Equation (165) can be obtained by using centroidal values

$[\mathrm{K}]=2 \pi[\overline{\mathrm{B}}]^{\mathrm{T}}[\mathrm{C}][\overline{\mathrm{B}}] \overline{\mathrm{r}} \Delta$

where

$\Delta=$ cross sectional area of the triangle ithe bar indicates evaluation at the element centroid).

Equation (166) has a further restriction in that the shape function [N] must be linear and does not require any additional nodal displacements other than at the element corners.

The shape function [N] can best be described by the geometry 
shown by Figure 23 using the nodes IJK. The purpose of [N] is to provide a means of interpolating displacements within the boundary of the element cefined ty the nodal displacements of the element corners.

In general, the displacement of any node, I, can be broken into two components, $u$ and $v$, as shown in Figure 23. A total displacement of six components is neeced to complete the nodal displacenent vector, $C$. For a given element,

$\{q\}=\left\{\begin{array}{c}u_{I} \\ v_{I} \\ u_{J} \\ v_{J} \\ u_{K} \\ v_{K}\end{array}\right\}$

Using a linear expression for $U_{I}$ and $v_{I}$, Equation (168) can be written for all the nodes,

$$
\begin{aligned}
& u_{I}=\alpha_{1}+\alpha_{2} r_{I}+\alpha_{3} z_{I} \\
& v_{I}=\alpha_{4}+\alpha_{5} r_{I}+\alpha_{6} z_{I} \\
& u_{J}=\alpha_{1}+\alpha_{2} r_{J}+\alpha_{3} z_{J} \\
& v_{J}=\alpha_{4}+\alpha_{5} r_{J}+\alpha_{6} z_{J} \\
& u_{K}=\alpha_{1}+\alpha_{2} r_{K}+\alpha_{3} z_{K} \\
& v_{K}=\alpha_{4}+\alpha_{5} r_{K}+\alpha_{6} z_{K}
\end{aligned}
$$

where

$$
r_{I}=\text { radial dimension of the nodal point I }
$$




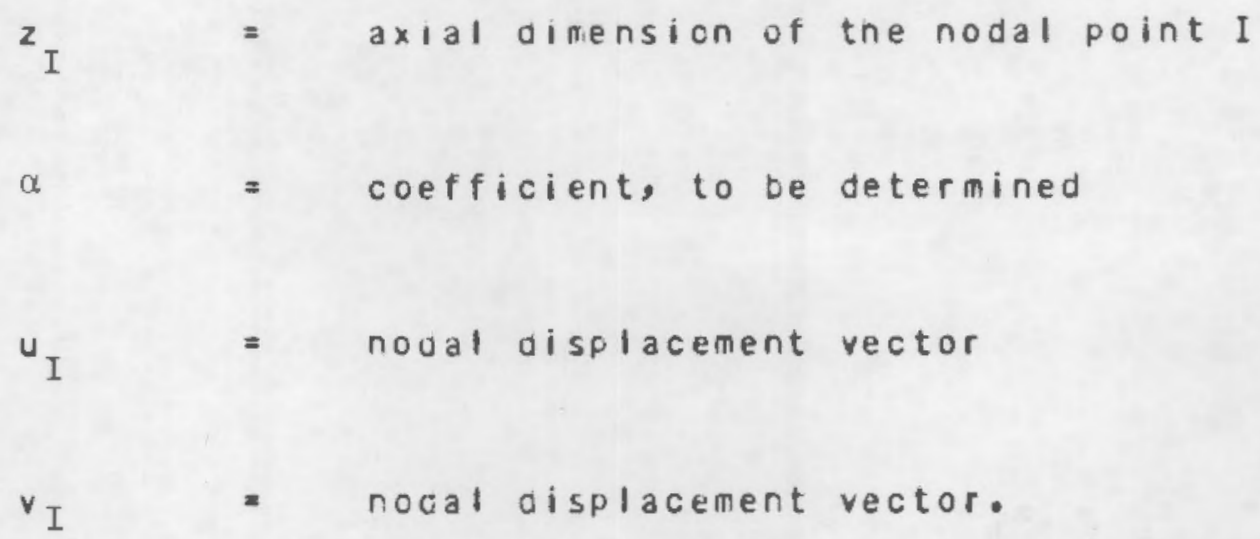

tquation (168) can be solved for the $\alpha$, and an expression can then be written for the displacements $u$ and $v$ of any point within the element at some location $r$ and $z$ [see Equation (169)]. This transtormation betmeen nodal point values and internal conditions is the interpolation function [N]

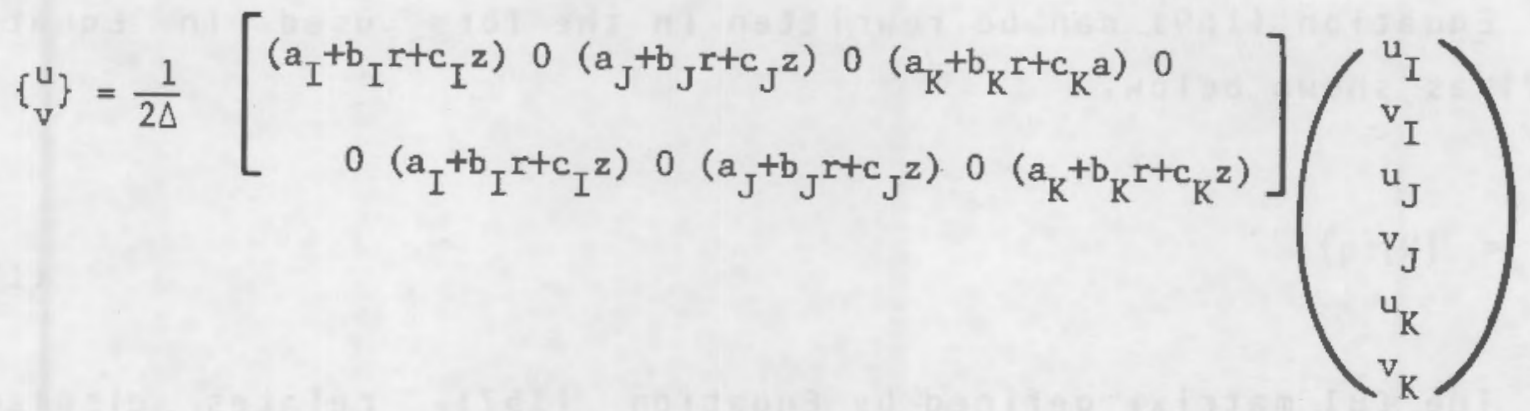

wnere

$$
\begin{aligned}
& \Delta=\text { area of the triangular cross section IJK } \\
& a_{I}=r_{J} z_{K}-r_{K} z_{J} \\
& a_{J}={ }^{r} z_{I}-{ }^{r_{I} z_{K}} \\
& a_{K}=r_{I} z_{J}-r_{J} k_{I} \\
& b_{I}=z_{J}-z_{K} \\
& b_{J}=z_{K}-z_{I}
\end{aligned}
$$




$$
\begin{aligned}
& b_{K}=z_{I}-z_{J} \\
& c_{I}=r_{K}-r_{J} \\
& c_{J}=r_{I}-r_{K} \\
& c_{K}=r_{J}-r_{I}
\end{aligned}
$$

Equation (169) can be rewritten in the form used in Equation $(157)$ as shown below.

$\left\{\begin{array}{l}u \\ v\end{array}\right\}=[N]\{q\}$

The [B] matrix, defined by Equation (157), relates displacements to strains and can be developed by examining the strain terms which are defined for the axisymetric condition as

$\{\varepsilon\}=\left\{\begin{array}{c}\varepsilon_{r} \\ \varepsilon_{\theta} \\ \varepsilon_{z} \\ \gamma_{r z}\end{array}\right\}=\left\{\begin{array}{l}\frac{\partial u}{\partial r} \\ \frac{u}{r} \\ \frac{\partial v}{\partial z} \\ \frac{\partial u}{\partial z}+\frac{\partial v}{\partial r}\end{array}\right\}$

(172) 
or, noting that $u$ and $v$ are $[q]$,

$\{\varepsilon\}=[B]\{q\}$

where $[\bar{B}]$ is evaluated at the element centroid (the tar denctes centroidal values for $r$ denoted oy $\bar{r}$ ),

$[\bar{B}]=\left[\begin{array}{cccccc}\frac{\partial N_{I}}{\partial r} & 0 & \frac{\partial N_{J}}{\partial r} & 0 & \frac{\partial N_{K}}{\partial r} & 0 \\ \frac{N_{I}}{\bar{r}} & 0 & \frac{N_{J}}{\bar{r}} & 0 & \frac{N_{K}}{\bar{r}} & 0 \\ 0 & \frac{\partial N_{I}}{\partial z} & 0 & \frac{\partial N_{J}}{\partial z} & 0 & \frac{\partial N_{K}}{\partial z} \\ \frac{\partial N_{I}}{\partial z} & \frac{\partial N_{I}}{\partial r} & \frac{\partial N_{J}}{\partial z} & \frac{\partial N_{J}}{\partial r} & \frac{\partial N_{K}}{\partial z} & \frac{\partial N_{K}}{\partial r}\end{array}\right]$

where

$$
\begin{aligned}
& \frac{\partial N_{I}}{\partial r}=b_{I} \quad \frac{\partial N_{I}}{\partial z}=c_{I} \\
& \frac{\partial N_{J}}{\partial r}=b_{J} \quad \frac{\partial N_{J}}{\partial z}=c_{J} \\
& \frac{\partial N_{K}}{\partial r}=b_{k}=c_{K} \\
& \frac{N_{I}}{r}=\frac{\partial N_{K}}{\bar{r}}+b_{I}+\frac{c_{I}}{\bar{r}}
\end{aligned}
$$




$$
\begin{aligned}
& \frac{\mathrm{N}_{J}}{\bar{r}}=\frac{a_{J}}{\bar{r}}+b_{J}+\frac{c_{J}^{\bar{z}}}{\bar{r}} \\
& \frac{N_{K}}{\bar{r}}=\frac{a_{K}}{\bar{r}}+b_{K}+\frac{c_{K} \bar{z}}{\bar{r}}
\end{aligned}
$$

The material matrix [C] can either be written for anisotsropic material properties or for isotropic conditions. PELET presentiy uses the latter condition in the cladding

$$
[C]=\frac{E}{(1+\mu)(1-2 \mu)}\left[\begin{array}{llll}
1-\mu & \mu & \mu & 0 \\
\mu & 1-\mu & \mu & 0 \\
\mu & \mu & 1-\mu & 0 \\
0 & 0 & 0 & \frac{1-2 \mu}{2}
\end{array}\right]
$$

where

$$
\begin{array}{ll}
E & =\text { elasic modulus } \\
\mu \quad & =\text { Poisson's ratio. }
\end{array}
$$

However in the fuel, the anisotropic form is used as follows

$$
C=\frac{E_{r}}{\left(1+\mu_{r}\right)\left(1-\mu_{r}-2 \frac{E_{r}}{E_{a}} \mu_{2}^{2}\right)}
$$




$$
\begin{array}{|llll|}
1-\frac{E_{r}}{E_{a}} \mu_{a}{ }^{2} & \mu_{r}+\frac{E_{r}}{E_{a}} \mu_{a}^{2} & \mu_{a}\left(1+\mu_{r}\right) & 0.0 \\
\mu_{r}+\frac{E_{r}}{E_{a}} \mu_{a}^{2} & 1-\frac{E_{r}}{E_{a}} \mu_{a}^{2} & \mu_{a}\left(1+\mu_{r}\right) & 0.0 \\
\mu_{a}\left(1+\mu_{r}\right) & \mu_{a}\left(1+\mu_{r}\right) & \left(1-\mu_{r}{ }^{2}\right) \frac{E_{a}}{E_{r}} & 0.0 \\
0.0 & 0.0 & 0.0 & \frac{\left(1+\mu_{r}\right)\left(1-\mu_{r}-2 \frac{E_{r}}{E_{a}} \mu_{a}{ }^{2}\right)}{2 \frac{E_{r}}{E_{a}}\left(1+\mu_{a}\right)}
\end{array}
$$

where

$$
\begin{aligned}
E & =\text { elastic modulus } \\
\mu & =\text { Poisson's ratio } \\
r & =\text { radial direction } \\
\text { a } & =\text { axial direction. }
\end{aligned}
$$

By using Equations (169), (171), (172), (174), and (175), a 11 of the inatrix formulation terms previously defined can be found. It 
is now necessary to develop the element stiffness matrix and load vectors for a single element. The implementation of this procedure for the radial and axial models is performed by formulating stiffness matrices for each individual element (xKBAR) and merging the separate matrices into a total stiffness matrix for the appropriate radial or axial model.

Assembly of the Global Stiffness Matrix-Radial Model--The assembly of indivioual finite elements into a total model involves careful accounting of the relative disflacements or degrees of freedon (DOF) of each element. The $r$ adial model shown in figure 24 has pairs of two triangular elements connected in series with adaitional pairs of elements. The merge operation of forming the global stiffness matrix from the element stiffness matrices consists of matching components in the individual stiffness matrices that involve common $O C F$ from other elements and then summing them.

Each individual element has six Dof or two displacements at each of three corner nodes. A combined or glooal stiffness matrix may contain several hundred or thousand cof. The individual matrices as well as the total global matrix are symmetric, bandeo and positive definite. The bandwidth is defined by the maximum number of DUF that interact with other Dof.

The fuel and cladding are assumed to be in contact, and a matrix for the total fuel-clacoing system is formed by doutlenumbering the DDF common to the two material regions. Figure 26 illustrates the the final merged system and Dof numbering for it.

Figures 27 ano 28 illustrates how the element stiffness components are assigned to the global stiffness matrix. Note that 


\section{NODE NUMBER}

8

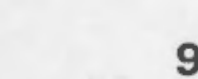

$9 \quad 10$

1112

13
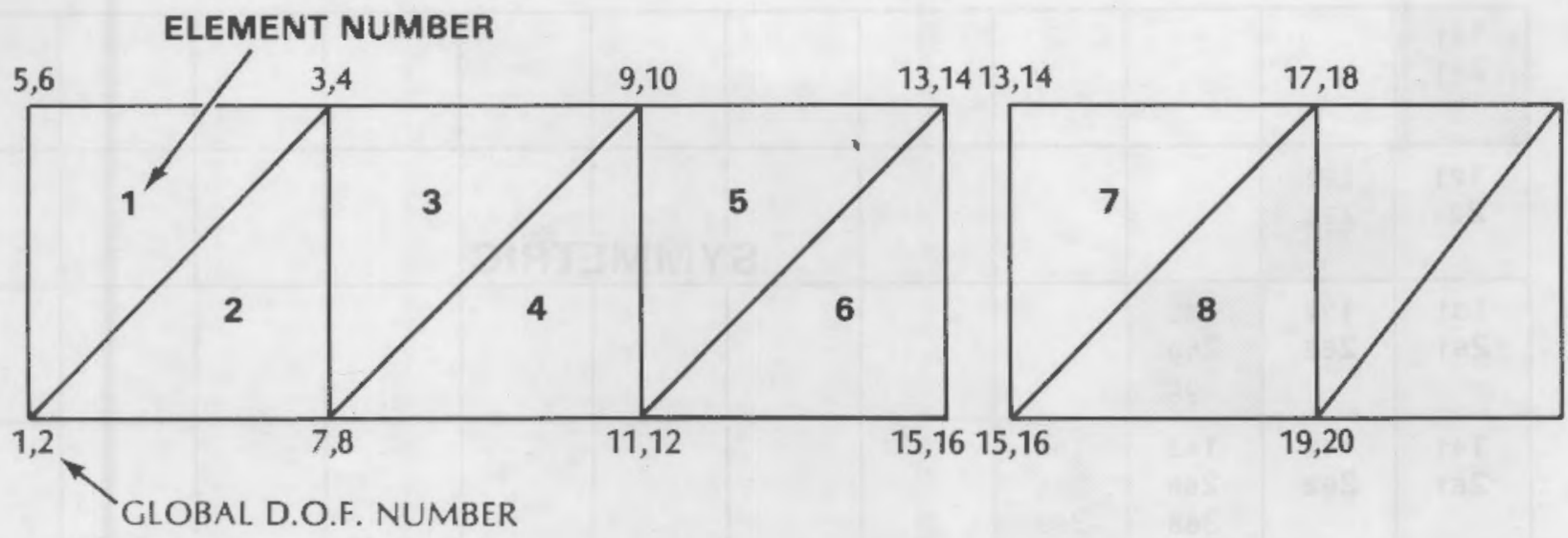

1

2

3

45

\section{FUEL-CLAD COMBINED MODEL}

Figure 26. Node and DOF numbering system for fuel-cladding combined radial model.

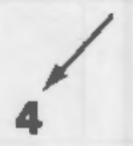

5

6

ELEMENT NUMBER

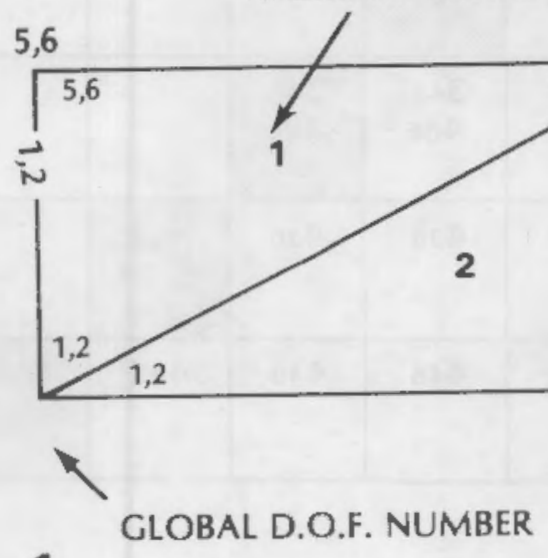

1

GLOBAL D.O.F. NUMBER

\section{FUEL OR CLAD SEPARATE MODEL}

Figure 27. Node and DOF numbering system for fuel or cladding separate radial model. 


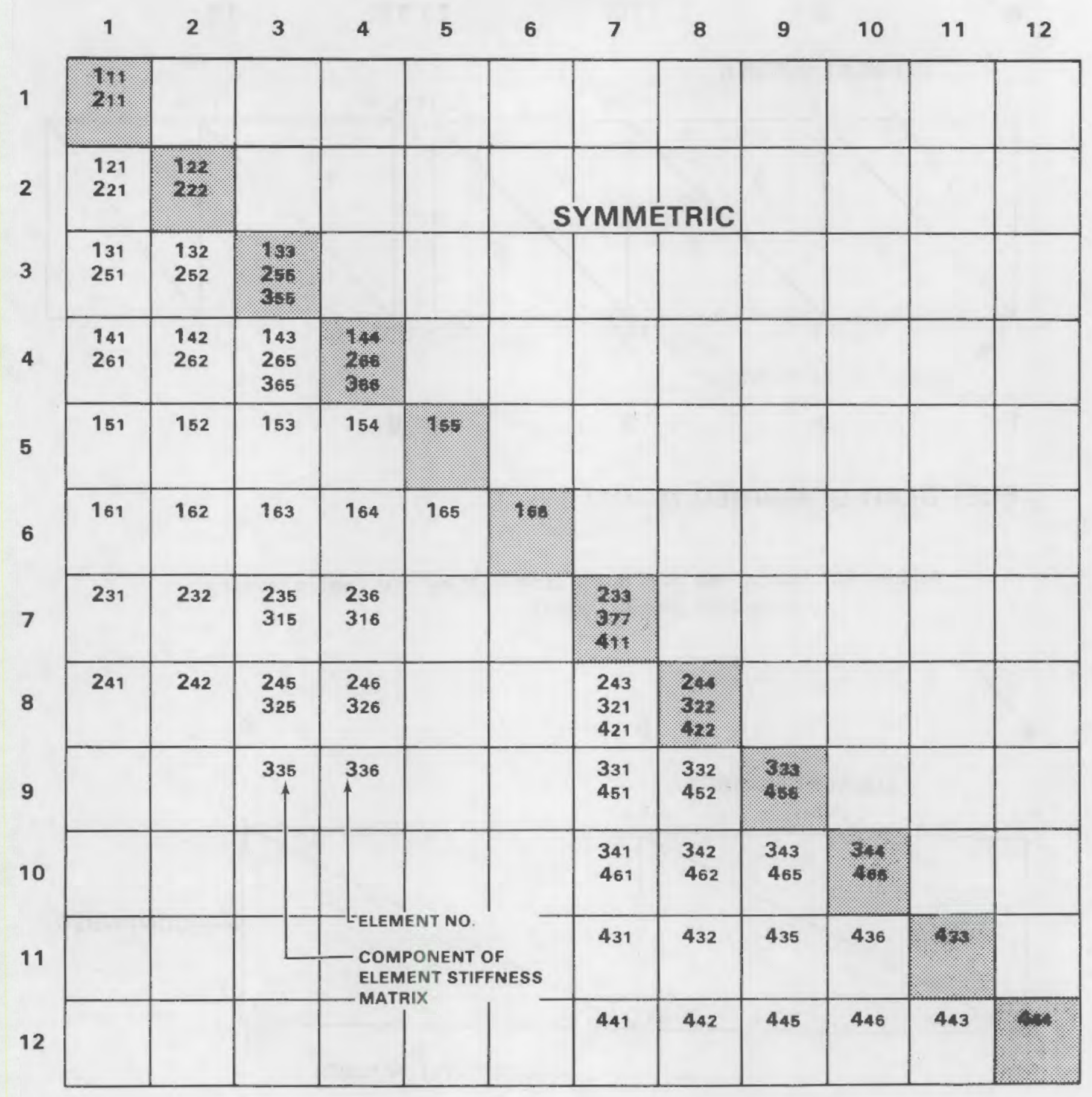

Flgure 28. Global stiffness matrix for the four elements in Figure 27, global DOF numbers are along the outside. 
node 1, for example, is shareo by elements 1 and 2. Therefore, some components from elements 1 and 2 will be present in the global inatrix in (global) DoF 1 and 2. Similarly node 5 is shareo by elements 1,2 , and 3 sc components from these elements would be expected to influence (global) DDF 3 and 4. The global stiffness matrix for the four elements shown in figure 27 is presented schematically in Figure 28. An entry I is meant to indicate the jk-th component of the element stiffness matrix for element I. The diagonal components from contributing elements appear on the diagonal of the global matrix at the DOF tnose elements share. The assignment of the off-diagonal element components is more suttle, but in every case the shared DOF can be observed to be properly influenced by the contributing elements; e.g., Dof 4 has offdiagonal influence from elements 1,2 , and 3 only. Also the global stiffness matrix can be observed to be banded and symmetric. Because of the symmetry, only the main diagonal and either the upper or lower codiagonals need be retained. This greatly reduces the required computer core storage as compareo with a square matrix of similar size.

Assembly of the Global Stiffness Matrix-Axial Model--The basic steps for calculating the element stiffness matrices used in the axial model are identical to the steps used in the radial model. The node and DOF numbering system for the axial model are shown in Figure 29. There is a renumbering of DOF in case of contact. The axial Dof for the boundaries of that region are to move together through the following procedure:

1. Let $i$ ano i be the two axial degrees of freedor to be locked. In the global stiffness matrix, row il is added to row i. To preserve symmetry, column i is adoed to column i. 


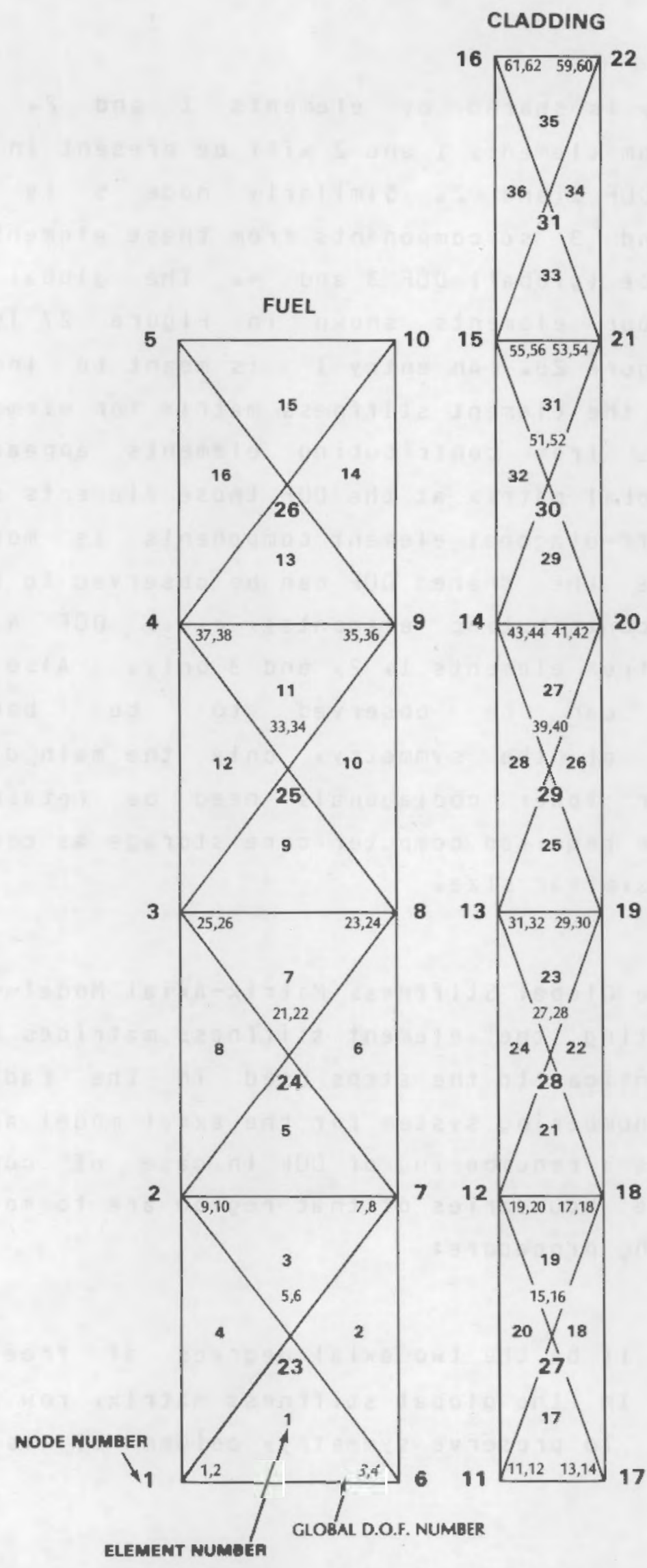

F1gure 29. Node and DOF numbering system for fuel and cladding combined axial model. 
2. Row and column il are filled with zeros and a 1.0 is inserted on the diagonal at position $(i i, i)$.

3. Load i is added to load $i$ and load i is filled with zeros.

4. In the solution, displacement ii will be zero and displacment $i$ Will represent the axial displacement in DOF $i$ and ii. Arbitrarily set displacement ii through displacement i.

This procedure has been shown to collect all the proper influence from contributing elements and consists of a series of simple algebraic row and column operations.

Application of Boundary Conditions--The only boundary condition in the models is zero axial displacement along the bottom. Accordingly, the rows and columins corresponding to those Dof are set to zero in the glotal matrix, the diagonal term is set to one, and the corresponding load is set to zero. This forces the proper displacements to be zero.

Formulation of the Load Vector--The last step in the formulation of the problem is to transform the right-hand side of Equation (163) into a series of nodal loads (vector f).

The first loao vector term in Equation (163) makes use of the [B] matrix to transform internal element initial strains into nodal loads, 


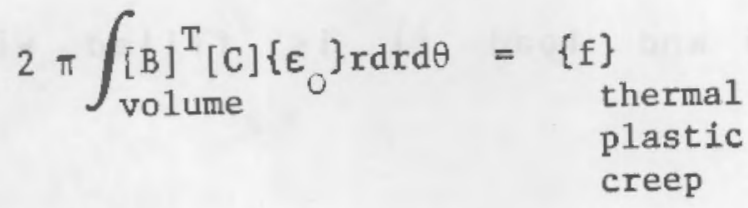

for centroidal values, this becomes

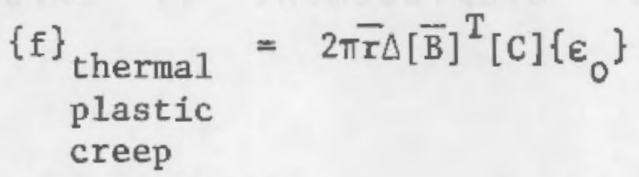

The external load vectors, fexternal, are cevelopec by integrating the surface tractions, or pressures, and developing equivalent nodal loads

$$
\int_{\text {surface }}^{[\mathrm{N}]^{\mathrm{T}} \mathrm{Tds}=\{\mathrm{f}\}} \text { external }
$$

Because of the geometry used, the external loads become equivalent to line loads which are pressure driven. This leads to

$\{f\}_{i_{\text {external }}}=\begin{aligned} & \text { Pressure Acting on } \\ & \text { Element Face } * \text { Face Area } \\ & \text { Total Length of Noda1 } \\ & \text { Line on Boundaries of } \\ & \text { Element Face (2 Nodes) }\end{aligned} * \begin{aligned} & \text { Length of } \\ & \text { Node Line (i) }\end{aligned}$

The same result is obtained as if the total force on the exterior surface of the element had been lumped at its exterior nodes. This process of lumping is performed throughout the program. 
solution procedure--Having formulated the global stiffness matrix and the loads, the remaining. step is to solve for displacements and translate these into element stresses and strains. The solution routine takes advantage of the banded symmetric nature of the stiftness matrix.

\section{Incrementation of Strains and Stresses-incremental} elastic strains and stresses are added to the total strain array after temperature convergence. Then creep/plastic strains are calculated and fed through the entire chain of radial and axial calculations to produce corrections to the stress and strain values. Automatic subdivision of the time step size may be done within this model to restrict the creep increment size. The solution is based on the concept that total strain can be described as

$E_{T}=\varepsilon_{\text {elastic }}+\varepsilon_{\text {creep }}+\varepsilon_{\text {thermal }}+\varepsilon_{\text {plast 1c }}$

and that for any one incremental step, the strains and associated load vectors will be separated as follows:

$$
\begin{aligned}
& \varepsilon_{T_{i}}=\varepsilon_{\text {elastic }}+\varepsilon_{\text {thermal }_{i}} \\
& \varepsilon_{T_{i+1}}=\varepsilon_{\text {elastic }}+\varepsilon_{\text {plastic }}+1 \\
& \varepsilon_{T_{1+2}}=\varepsilon_{\text {elastic }}+\varepsilon_{\text {creep }}+2
\end{aligned}
$$

a. The elastic stresses and strains for the it 1 and it? steps, change the elastic stress and strain distributions obtained as a result of the imposedplastic or creep strains. The total strain at the end of step will be a sum of $\varepsilon_{T i}+\varepsilon_{T i}+$ + $\varepsilon_{T i} 2$ with external loads and thermal loads from the ETi increment and the plastic and creep effects from the $i+1$ and $i+2$ increments. 
The stress state can be computed at the conclusion of a given time step or load step and the corrections to the stress distribution is as follows?

\section{Ibecma1_t_ELastic}

$\{\sigma\}_{1}=[C]\left\{\varepsilon_{T}\right\}-[C]\{\varepsilon\}_{\text {thermal }}$

Creeg

$\{\sigma\}_{1}=[C]\left\{\varepsilon_{T}\right\}-[C]\{\varepsilon\}_{\text {creep }}$

Plastic

$\{\sigma\}_{i}=[C]\left\{\varepsilon_{T}\right\}-[C]\{\varepsilon\}_{\text {plastic }}$

$(184)$

where the total strain and stress vectors for the current point in time is a summation of the individual components.

$$
\begin{aligned}
& \left\{\varepsilon_{\text {total }}\right\}=\sum_{i=1}^{n}\left\{\varepsilon_{T}\right\}_{i} \\
& \left\{\sigma_{\text {total }}\right\}=\sum_{i=1}^{n}\{\sigma\}_{i}
\end{aligned}
$$

Anisotropic Behavior--Anisotropy in plastic and creep deformation has a significant effect on the resulting deformation. The anisotropy model that is used is based on Hill's ${ }^{26}$ concept of changing the definition of the effective stress and strain terms, and in addition, changing the coefficients in the Prandti-Reuss equations. The modified Prandti-Reuss equations 
allocate the inelastic strain increment normal to the yield surface as a function of the stress and strain.

The definition of the anisotropy parameters change as a function of direction of testing. The equations for this application are referenced to the axial direction. This means that anisotropic strain characterizations should be based on uniaxialaxial material test results. A similiar set of equations can be developed for any of the other three directions.

For the isotropic case, the effective stress is given by

$\left.\sigma_{e}=\frac{1}{2}\left[\sigma_{11}-\sigma_{22}\right)^{2}+\left(\sigma_{22}-\sigma_{33}\right)^{2}+\left(\sigma_{33}-\sigma_{11}\right)^{2}\right]^{1 / 2}$

where

$\begin{aligned} 1 & =\text { axial direction } \\ 2 & =\text { hoop direction } \\ 3 & =\text { radial direction. }\end{aligned}$

The effective strain is given by

$$
d \varepsilon_{e}^{P}=\sqrt{\frac{2}{3}}\left[\left(d \varepsilon_{11}-d \varepsilon_{22}\right)^{2}+\left(d \varepsilon_{22}-d \varepsilon_{33}\right)^{2}+\left(d \varepsilon_{33}-d \varepsilon_{11}\right)^{2}\right]^{1 / 2}
$$

The PrandtI-Reuss equations are 


$$
\begin{aligned}
d \varepsilon_{e}^{P} & =\frac{d \varepsilon_{P}}{\sigma_{e}}\left[\sigma_{11}-1 / 2\left(\sigma_{22}+\sigma_{33}\right)\right] \\
d \varepsilon_{e}^{P} & =\frac{d \varepsilon_{P}}{\sigma_{e}}\left[\sigma_{22}-1 / 2\left(\sigma_{11}+\sigma_{33}\right)\right] \\
d \varepsilon_{e}^{P} & =\frac{d \varepsilon_{P}}{\sigma_{e}}\left[\sigma_{33}-1 / 2\left(\sigma_{22}+\sigma_{11}\right)\right]
\end{aligned}
$$

For the case of anisotropy referenced to the axial direction, the eftective stress is given by

$$
\sigma_{e}=\left[\frac{R}{R+1}\left(\sigma_{11}-\sigma_{22}\right)^{2}+\frac{R}{P(R+1)}\left(\sigma_{22}-\sigma_{33}\right)+\frac{R}{R+1}\left(\sigma_{33}-\sigma_{11}\right)\right]^{l / 2}
$$

where

$$
\begin{array}{ll}
\mathrm{R}=\frac{\delta \varepsilon_{\text {hoop }}}{\delta \varepsilon_{\text {radial }}} & \text { (obtained from a uniaxial axial test) } \\
\mathrm{P}=\frac{\delta \varepsilon_{\text {axial }}}{\delta \varepsilon_{\text {radial }}} & \text { (obtalned from a uniaxial hoop test) }
\end{array}
$$$$
\delta \varepsilon=\text { an increment of creep or plastic strain }
$$

The effective strain is given by

$$
\begin{aligned}
d \varepsilon_{e}^{P}= & \frac{l}{G}\left[a_{1}\left(a_{2} d \varepsilon_{11}-a_{3} d \varepsilon_{22}\right)^{2}+a_{2}\left(a_{3} d \varepsilon_{22}-a_{1} d \varepsilon_{33}\right)^{2}\right. \\
& \left.+a_{3}\left(a_{1} d \varepsilon_{33}-a_{2} d \varepsilon_{11}\right)^{2}\right]^{1 / 2}
\end{aligned}
$$


where

$$
\begin{aligned}
& G=\frac{R^{2}+R+P R}{P(R+1)^{2}} \\
& a_{1}=\frac{R}{R+1} \\
& a_{2}=\frac{R}{P(R+1)} \\
& a_{3}=\frac{1}{R+1}
\end{aligned}
$$

The modified Prandt-Reuss flow equations are

$$
\begin{aligned}
& \mathrm{d} \varepsilon_{33}^{\mathrm{P}}=\frac{\mathrm{d} \varepsilon_{\mathrm{P}}}{\sigma_{\mathrm{e}}}\left[\frac{\mathrm{P}+\mathrm{R}}{\mathrm{P}(1+\mathrm{R})} \sigma_{33}-\frac{\mathrm{R}}{\mathrm{P}(1+\mathrm{R})} \sigma_{22}-\frac{1}{1+\mathrm{R}} \quad \sigma_{11}\right] \\
& \mathrm{d} \varepsilon_{22}^{\mathrm{P}}=\frac{\mathrm{d} \varepsilon_{\mathrm{P}}}{\sigma_{\mathrm{e}}}\left[\frac{\mathrm{R}(\mathrm{P}+1)}{\mathrm{P}(\mathrm{R}+1)} \quad \sigma_{22}-\frac{\mathrm{R}}{\mathrm{P}(\mathrm{R}+1)} \quad \sigma_{33}-\frac{\mathrm{R}}{\mathrm{R}+1} \quad \sigma_{\mathrm{II}}\right] \\
& \mathrm{d}_{11}^{\mathrm{P}}=\frac{\mathrm{d} \varepsilon_{\mathrm{P}}}{\sigma_{\mathrm{e}}}\left[\sigma_{11}-\frac{\mathrm{R}}{\mathrm{R}+1} \sigma_{22}-\frac{1}{\mathrm{R}+1} \sigma_{33}\right]
\end{aligned}
$$

For the case here the material is isotropic, $R=1, P=1$, and Equations (190), (191), and (192) become equal to Equations (187), $(138)$, and $(189)$.

Creep and Plasticity Equations--After the incremental elastic stresses and strains have been found and the total stress and strain arrays updated accordingly, incremental creep (generalized) strains are calculated ano translated into loads, and thus, into stressstrain increments via the same procedure used for thermal strains. 
An array of incremental strains is calculated by $d E_{e}^{P}$ using eitner a strain hardening or time-hardening option (see figure 30 ). The procedure is very general in nature and does not require a closed solution explicit in strain. The solution for a strain hardening calculation uses a modified Newton-Raphson iteration technique.

The modified Prandtl-Reuss equations are used to develop

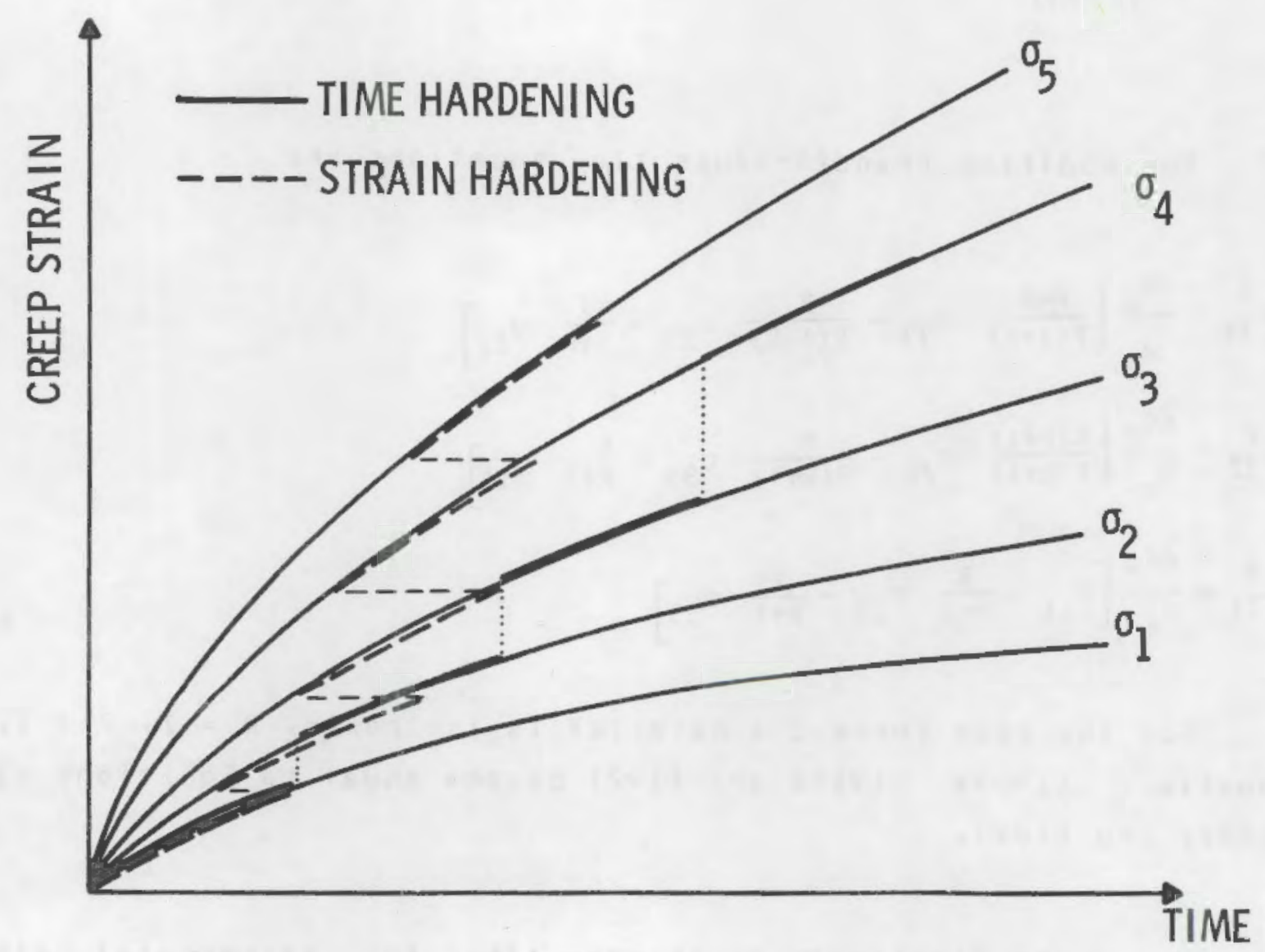

F1gure 30. Comparison of strain hardening and time hardening options available. 
initial strain vectors for each element $\left[\varepsilon_{0}\right]$. Using Equation (179), a set of of creep load vectors are developed that, when appliea to the finite element structure, will cause it to deform. The computed strains are the tctal strains caused by the increments of creep strain. The corrections to the total stress state are found using Equation $(184)$.

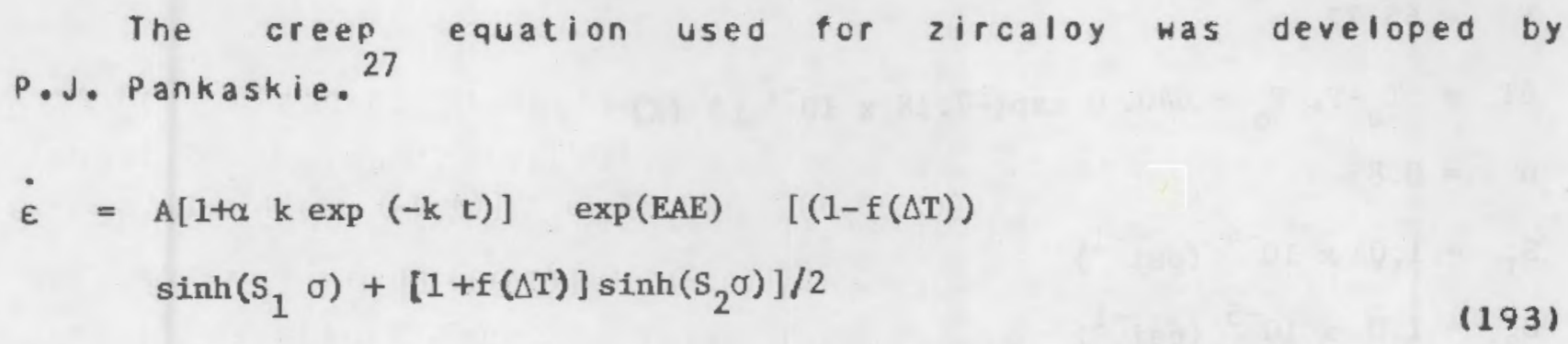

The creep equation used for zircaloy was developed by P.J. Pankaskie. ${ }^{27}$

$\dot{\varepsilon}=A[1+\alpha k \exp (-k t)] \quad \exp (E A E) \quad[(1-f(\Delta T))$

$\left.\sinh \left(S_{1} \sigma\right)+[1+f(\Delta T)] \sinh \left(S_{2} \sigma\right)\right] / 2$

(193)

where $E A E$ is the effective activation energy consisting of the sum of a high temperature thermal activiation energy term, TAE, and a fast flux or low temperature activation energy term, $F A E$, as follows:

$$
\begin{aligned}
& \mathrm{TAE}=-[\mathrm{C}(1-\mathrm{f}(\Delta \mathrm{T}))+(\mathrm{D}-0.038 \sigma) \quad(1+\mathrm{f}(\Delta \mathrm{T}))] / 2 \mathrm{R} \mathrm{T} \\
& \mathrm{FAE}=0.5 \quad(\mathrm{E}-\mathrm{F}) \quad(1+\mathrm{f}(\Delta \mathrm{T})) \\
& \phi>0, \mathrm{E}=\mathrm{n} \cdot \ln \phi \\
& \phi=0, \mathrm{E}=(\mathrm{C}-0.044 \sigma)[1-\Delta \mathrm{T} /(\mathrm{RT})] /\left(2 \mathrm{RT}_{\mathrm{o}}\right) \\
& \Delta \mathrm{T}<0, \mathrm{f}(\Delta \mathrm{T})=-1 \\
& \Delta \mathrm{T} \geq 0, \mathrm{f}(\Delta \mathrm{T})=+1
\end{aligned}
$$




$$
\begin{aligned}
& \alpha=3310(\mathrm{hr}) \\
& \mathrm{k}=0.0044\left(\mathrm{hr}^{-1}\right) \\
& A=5.96 \times 10^{14}\left(\mathrm{hr}^{-1}\right) \\
& \mathrm{C}=63600.0\left(\mathrm{cal} / \mathrm{mole} \mathrm{e}^{\circ} \mathrm{K}\right) \\
& \mathrm{D}=9500.0\left(\mathrm{cal} / \mathrm{mole} \mathrm{e}^{\circ} \mathrm{K}\right) \\
& \mathrm{F} \quad=65.77 \\
& \Delta \mathrm{T}=\mathrm{T}_{0}-\mathrm{T}, \mathrm{T}_{0}+640.0 \exp \left(-7.18 \times 10^{-7} \sigma\right) \\
& \mathrm{n} \quad=0.85 \\
& \mathrm{~s}_{1}=1.04 \times 10^{-4}\left(\mathrm{psi}^{-1}\right) \\
& \mathrm{s}_{2}=1.0 \times 10^{-5}\left(\mathrm{psi}^{-1}\right) \\
& \phi \text { - fast flux - avt ( } \mathrm{z} \geq 1 \mathrm{Mev}) \\
& \sigma \quad=\text { applied stress (psi) }
\end{aligned}
$$

Plasticity--The calculation of plastic strains is based on using the same initial strain method as is used for the creep calculation. The solution procedure, however, is implemented in a different manner and the criteria controlling the calculation are significantly altered.

The plasticity loop calculation is performed at the start of a creep loop. It is exercised when the stresses obtained from the just-completed elastic load step have driven the stresses in one or more elements above the yield stress. A test is made for each element based on the temperature and generalized strain in each element for that time.

As an example, consider the four-element cladding model shown 
in Figure 27. For some loading conditions, the stresses for this four-element model are shown in figure 31. At the conclusion of the just-completed elastic step, the stresses are shown to be above the yield stress curve. The stresses are then reduced back to the starting point shown by the stress conditions below the line. The total load step is then divided into substeps such that the first substep just brings the most plastic element to the yield surface. At this point, a second load substep is taken that will bring the second element to the yield surface. At the completion of this substep, a corrective plastic strainincrement is computed using the initial strain load vector. The strain that is incurred by element 4 as it leaves the yield surface is considered as plastic for both the pseudo-elastic and plastic portions. This procedure is repeated until all four elements are plastic as is shown when

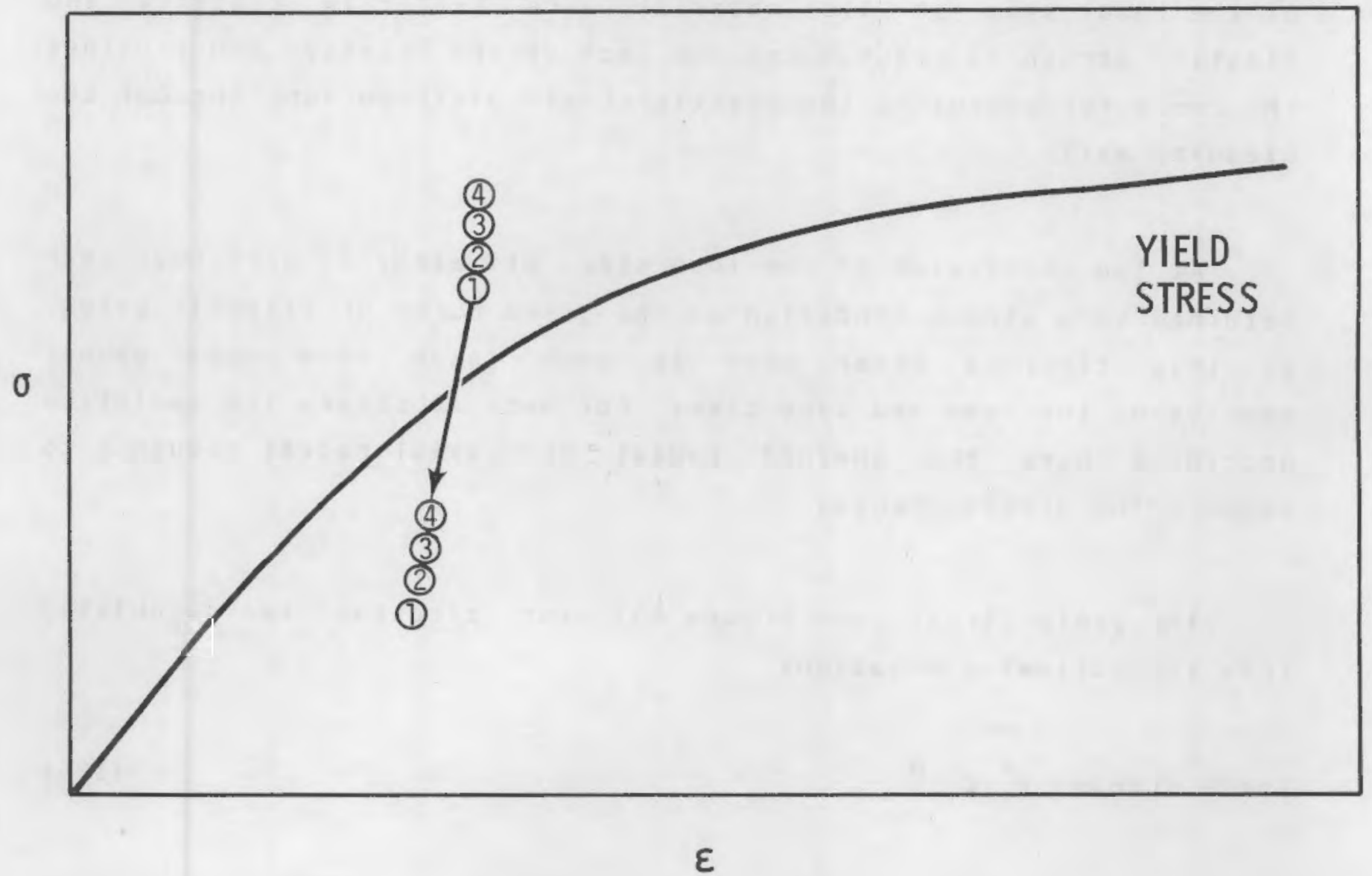
Figure 31. Elements $1,2,3$ and 4 are plastic and are brought
back prior subplastic state. 
element 1 is trought to the yield surface. A corrective plastic load step is taken and then the final portion of the original load step can be taken driving all elements above the yield surface. This procedure is concluded with a final plastic load step to correct the stress back to the yield condition (see figure 32 ).

In actual practice, the oscillation of the elements below the yield surface after a plastic corrective substep is less than 3.45 MPa out of 275.8 to $413.7 \mathrm{MPa}$. The actual spread between stresses decreases as more plastic substeps are taken and in the limit, elements 1 through 4 would be at the same stress at the conclusion of the load stef if the material were perfectly plastic. The plastic strain is accumulated for each of the substeps and provides the basis for computing the plastic strain distributions through the cladding wall.

At the conclusion of the load step, all elements will have teen returned to a stress condition on the yield curve or slight ly below. At this time, a creep step is then taken in a normal manner completing the $10 a 0$ and time step. For each substep, the solution procedure uses the chained radial and axial radial sequence to compute the oisplacenents.

The yield stress (see figure 33 ) for zircaloy is calculated from the following equation:

yield stress $=c \sigma^{n}$ 


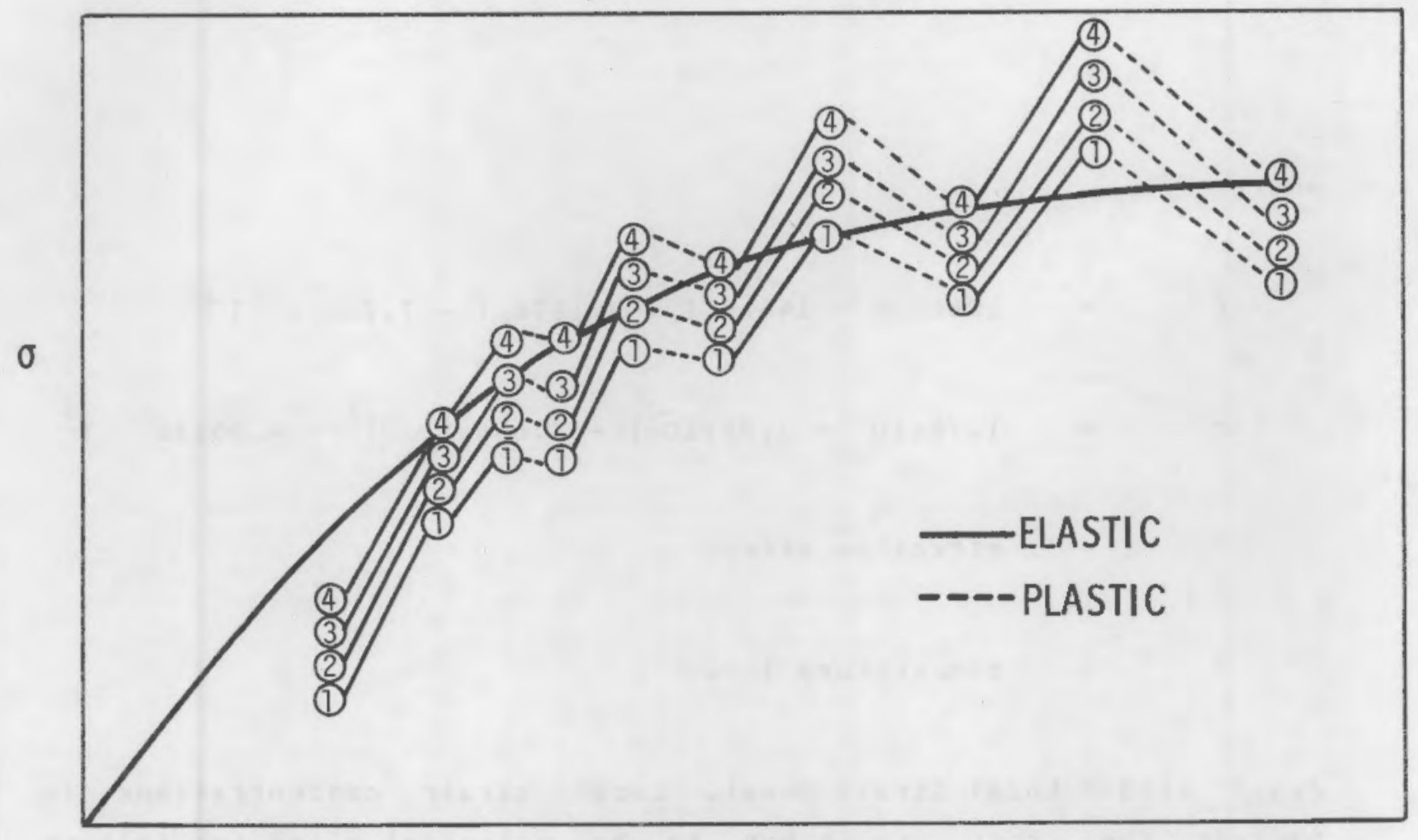

$\varepsilon$

Figure 32. The plastic strain taken by alternating plastic
elastic steps

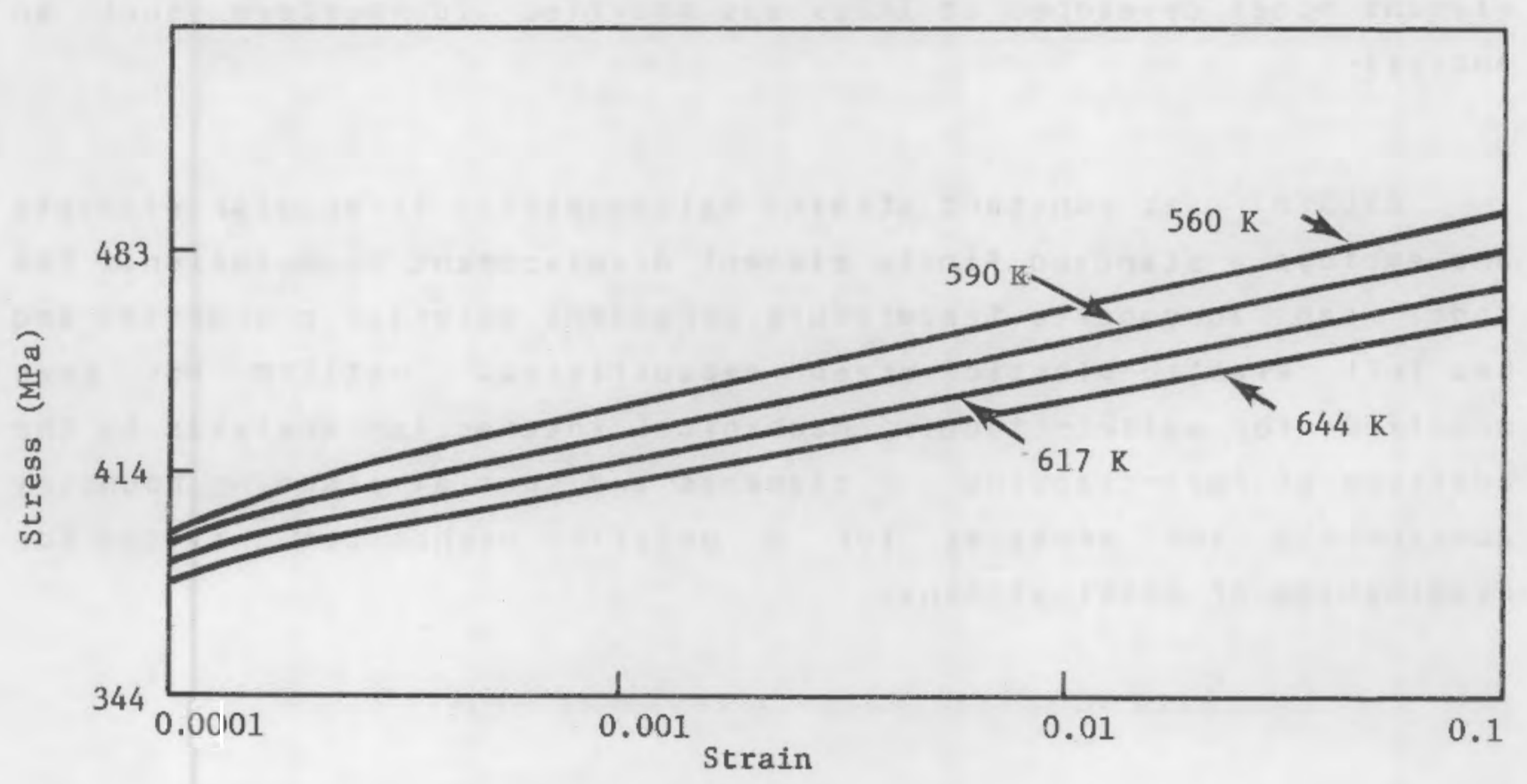

Figure 33. Yield stress versus strain used in GAPCON-3 calculations for temperature range $560 \mathrm{~K}$ to $644 \mathrm{~K}$. 
where

$\begin{aligned} C & =12860.0-146.5 \mathrm{~T}+0.1378 \mathrm{~T}^{2}-7.28 \times 10^{-5} \mathrm{~T}^{3} \\ n \quad & =1.75 \times 10+3.58 \times 10^{-2} \mathrm{~T}-7.638 \times 10^{-4} \mathrm{~T}^{2}-4.95 \times 10^{-7} \mathrm{~T}^{3} \\ \sigma \quad & \text { effective stress } \\ T \quad & \text { temperature (K). }\end{aligned}$

2.4.3 AXISYM Local Strain Model. Local strain concentrations in nuclear fuel rods are known to be potential sites for failure initiation. Assessment of such strain concentrations requires a two-dimensional analysis of stress and strain in both the fuel and the clading during pellet-cladding mechanical interaction. To provide this capability in the FRAPCON-2 code, AXISYM, a finite element model developed at INEL, was modified to perform such an analysis.

AXISYM uses constant strain, axisymmetric, triangular elements and employs a standard finite element displacement formulation. The model can accomodate temperature dependent material properties and has full elastic-plastic creep capabilities. AXISYM has been modified for pellet-cladding mechanical interaction analysis by the addition of fuel-cladding gap elements and special cladoing boundary constraints and provides for a detailed mechanical analysis for examination of local strains. 
2.5 Fuel Rod Internal Gas Pressure Response

After the fuel rod temperature and deformation calculations have been completed, the pressure of the gas in the fuel rod is computea. To calculate the gas pressure, the temperature ano volume of the gas are required. The thermal models discussed in Section 2.3 proviae the temperature of the gas in the fuel rod plenum, fuel-cladding gap, and fuel voids. The deformation models discussea in section 2.4 provide information for computing the volume of the fuel rod plenum, fuel-cladding gap, ano fuel voids.

The fuel roc internal gas pressure model is tased on the following assumptions:

1. Perfect gas law holds (PV = NRT).

2. Gas pressure is constant throughout the fuel rod.

3. Gas in the fuel cracks is at the average fuel temperature.

2.5.1 Fuel Rod Internal Gas Pressure. Fuel rod internal gas pressure is computeo from the perfect gas law modified to permit. different volumes of gas at oifferent temperatures as given by

$P=\frac{N R}{\sum \frac{V_{i}}{T_{i}}}$ 
where
$p$
- system pressure of gas (Pa)
$N$
$=$ moles of gas in fuel roo (gm.mole)
R
$=$ universal gas constant (k.gmemole)
$V=$ the $i-t h$ volume $\left(m^{3}\right)$
I = temperature of gas in the i-th volume $(K)$.

In FRAPCON-2, the different volumes considered are dish, crack, porosity, plenum, fuel-cladding gap and roughness volume. The choice of mechanics options, PELET Or FRACAS, will result in the total void volume being apportioned between fuel cracks and fuelclading gap in oifferent proportions.

Based on the above discussion, the detailed gas lam becomes

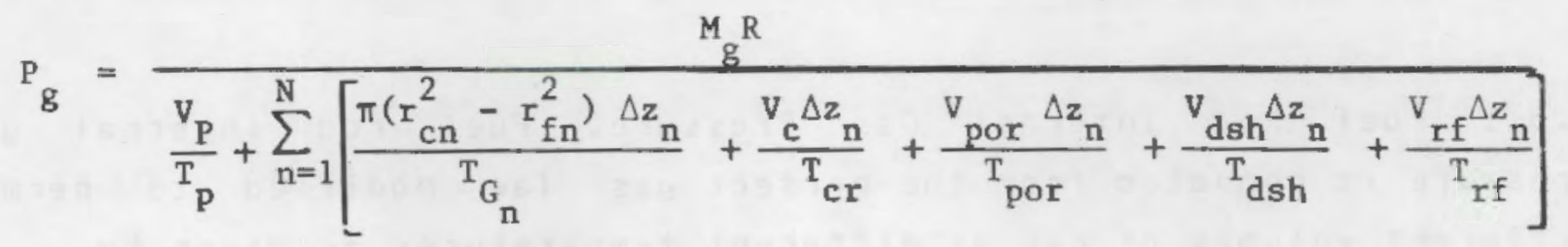

where

$P_{g} \quad=$ internal fuel rod pressure $(P a)$ 


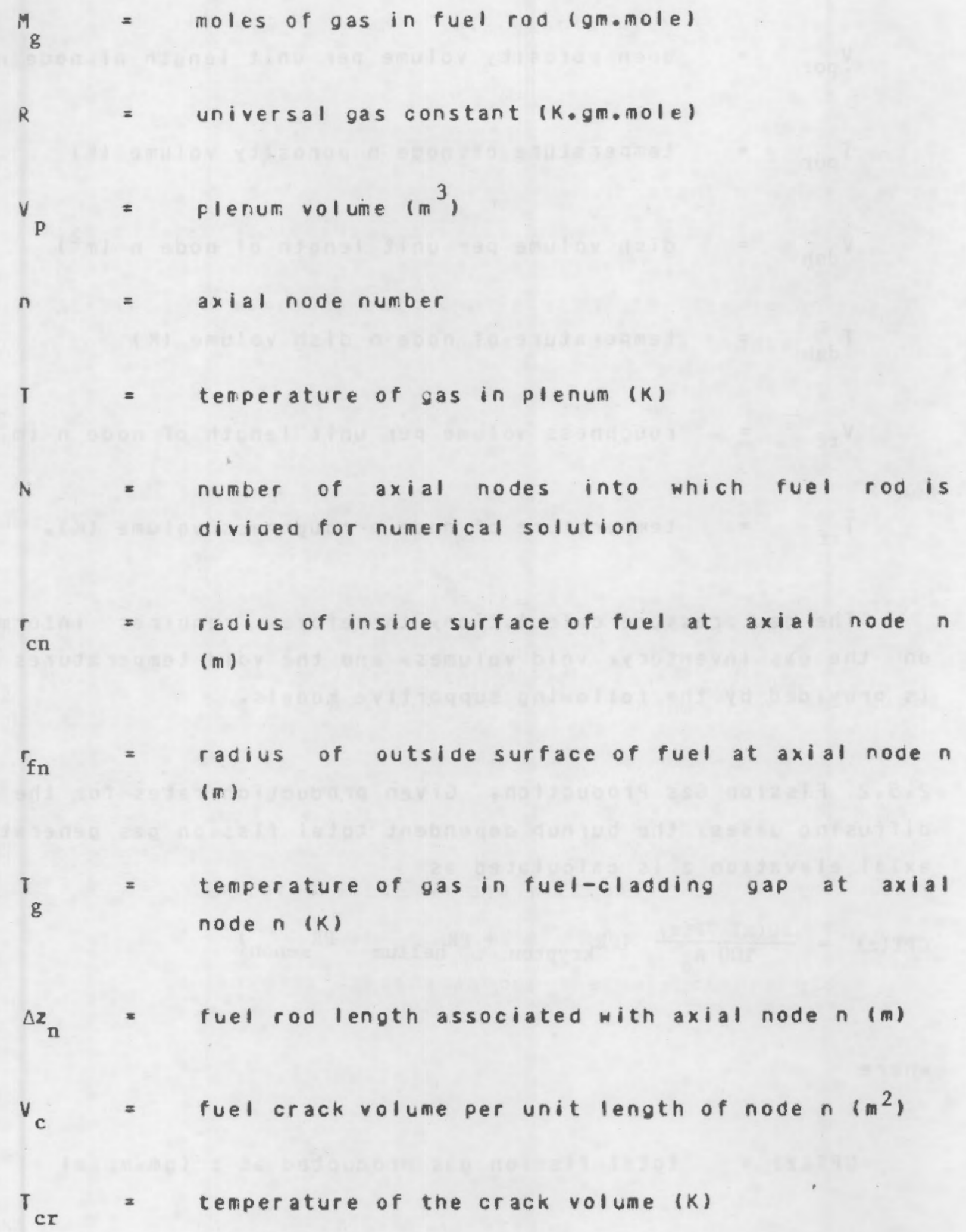




$$
\begin{aligned}
& v_{\text {por }}=\text { open porosity volume per unit length of node } n\left(m^{2}\right. \text { ) } \\
& T_{\text {por }}=\text { temperature of node } n \text { porosity volume }(k) \\
& v_{\text {dsh }}=\text { dish volume per unit length of node } n\left(m^{2}\right) \\
& T_{\text {dsh }}=\text { temperature of node } n \text { dish volume }(k) \\
& v_{r f}=\text { roughness volume per unit length of node } n\left(m^{2}\right) \\
& T_{r f}=\text { temperature of node } n \text { roughness volume }(k) .
\end{aligned}
$$

The gas pressure calculation, therefore, requires information on the gas inventory, void volumes, and the void temperatures which is provided by the following supportive models.

2.5.2 Fission Gas production. Given production rates for the major diffusing gases, the burnup dependent total fission gas generated at axial elevation $z$ is calculated as

$\operatorname{GPT}(z)=\frac{B U(z) V F(z)}{100 A_{v}}\left(P_{\text {krypton }}+P_{\text {hellum }}+P_{\text {xenon }}\right)$

where
GPT $(z)=$
total fission gas producted at z (gm.mole)
$B U(z)=$ burnup at 2 (fission/cc) 


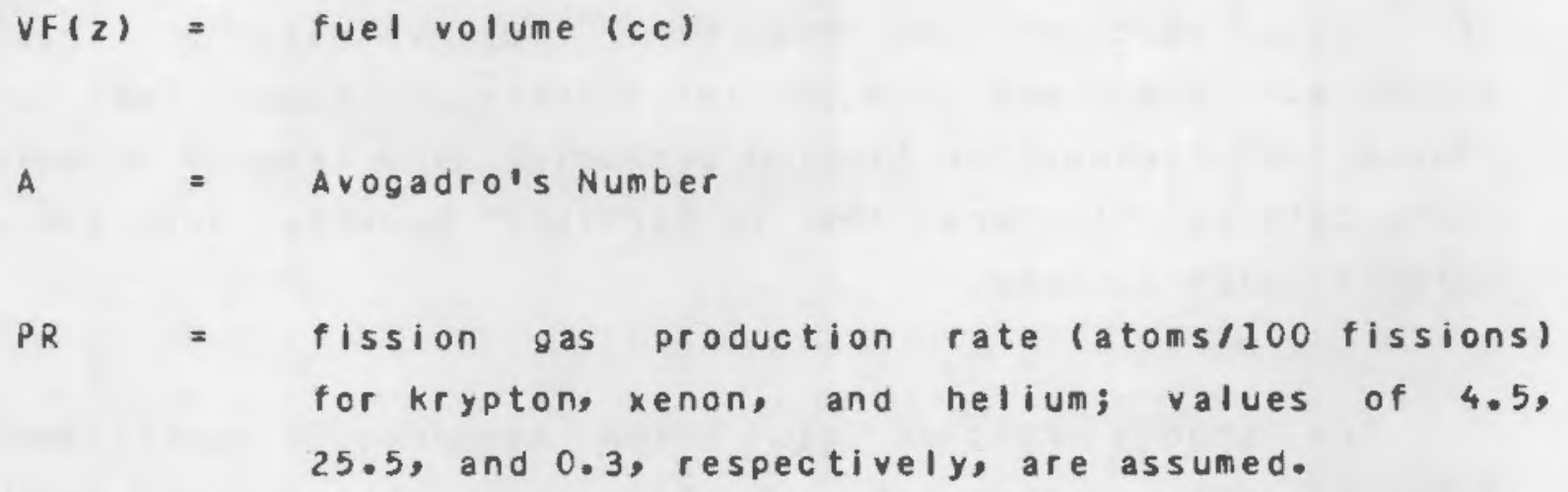

All the fission gas produced, however, is not releaseo. A portion is trapped in the fuel and a portion is released to the fuel-cladoing gap volume. Only the releasedportion is used to calculate the rod internal gas pressure. The gas release fraction is calculated as discussed in the following sections.

2.5.3 Fuel Rod Gas Release. Gas release models in FRAPCON-2 account for not only fission gas release (krypton, xenon, ano helium) but also nitrogen release. The nitrogen is released from the fuel lattice where it is trapped during the fuel fabrication process. Fission gas release in FRAPCON-2 includes five model options: ANS-5.4, ${ }^{28}$ Beyer-Hann, ${ }^{29}$ MacDonald-Weisman, 30 FAST-GRASS, and GRASS. 31 Each of these release models is discussed below.

2.5.3.1 ANS-5.4 Gas Release Model--The ANS-5.4 fractional fission gas release is calculated (on a local basis only) as a function of time and fuel temperature and burnup. The fuel is divided into $r$ adial and axial nodes according to the proposed ANS standard. A user requirement is that the time step sizes be such that the burnup increments do not exceed 2000 MWd/MTU.

The modeling is oivided into two main sections, one for release 
of stable isotopes and the other for release of short-lived isotopes. There are high ana low temperature models for toth the stable and radioactive fission products. The release is calculated using both the nigh and low temperature models, and the larger release value is useo.

The stable fission gas (high temperature model) cumulative total release fraction for a fuel volume after time step $k$ is defineo as

$F_{K}=1-\left\{\sum_{i=1}^{K-1}\left[B_{i}\left(\tau_{i} g_{i}-\tau_{i+1} g_{i+1}\right) / D_{i}^{\prime}\right]+B_{K} \Delta t_{K} g_{K}\right\} / \sum_{i+1}^{K} B_{i} \Delta t_{i} \cdot$

with

$\tau_{i}=\sum_{j=1}^{K} D_{j}^{\prime} \Delta t$

$g_{1}=g\left(\tau_{i}\right)=1-4 \sqrt{\tau_{1} / \pi}+3 \tau_{1} / 2 \quad$ for $\tau_{1}<0.1$

12001

$g_{1}=g\left(\tau_{i}\right)=\frac{1}{15 \tau_{i}}-\frac{6}{\tau_{i}}\left(\sum_{n=1}^{3} \frac{\exp \left(-n^{2} \pi^{2} \tau_{1}\right)}{n^{4} \pi^{4}}\right)$ for $\tau_{1}>0.1$

$D_{i}^{\prime}=\left[\left(D_{0} / a^{2}\right) \exp \left(-Q / R T_{i}\right)\right] \times 100^{B u_{1} / 28000}$ 


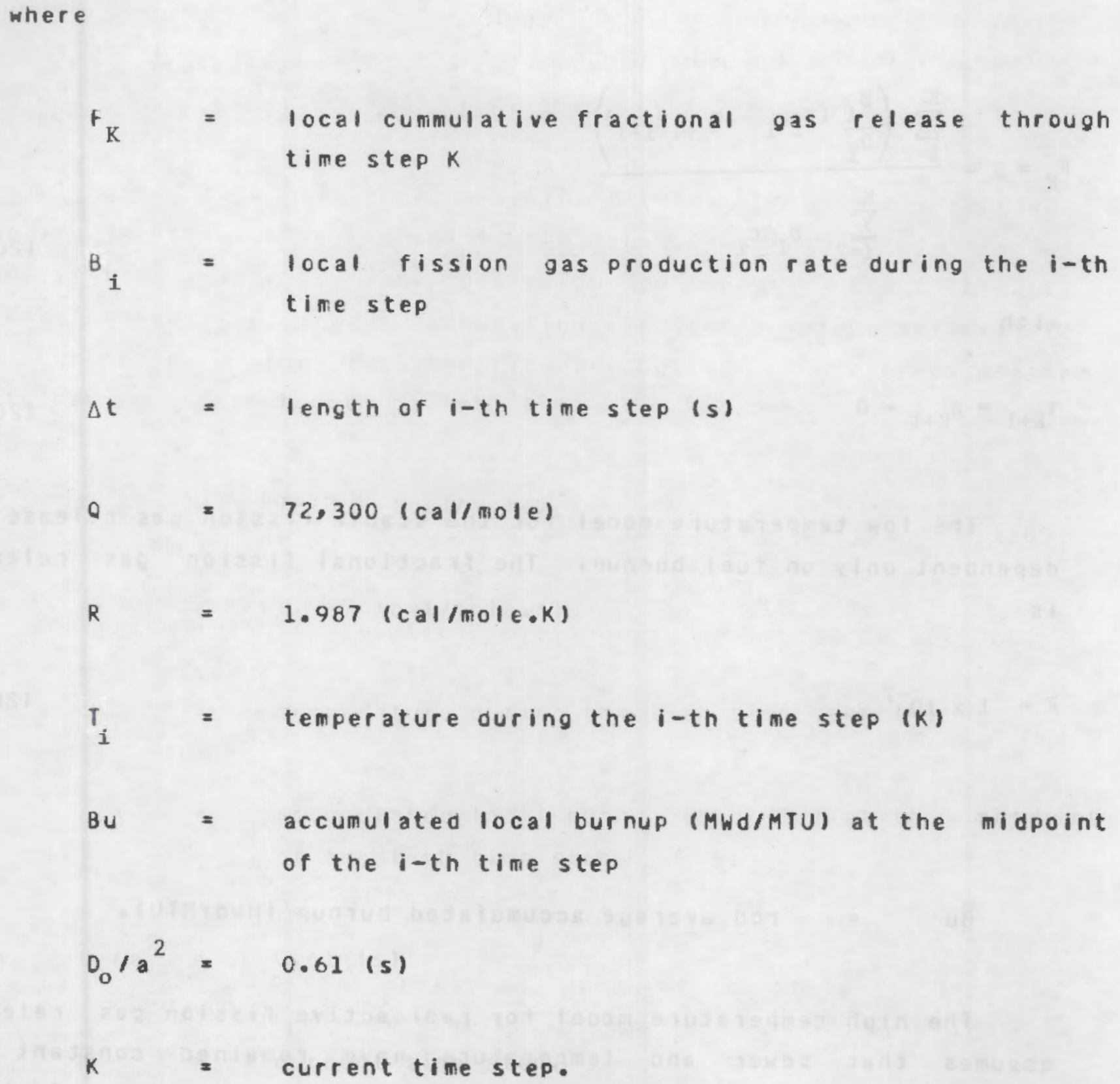


$F_{K}=1-\frac{\sum_{i=2}^{K}\left(\frac{B_{i}}{D_{i}}\left(\tau_{i} g_{1}-\tau_{i+1} g_{i+1}\right)\right)}{\sum_{i=2}^{K} B_{i} \Delta t_{i}}$

with

$\tau_{\mathrm{K}+1}=\mathrm{g}_{\mathrm{K}+1}=0$

The low temperature model for the stable fission gas release is dependent only on fuel burnup. The fractional fission gas release is

$\mathrm{F}=1 \times 10^{-7} \mathrm{Bu}$

where

Bu = rod average accumulated burnup (MWd/MTU).

The high temperature model for radioactive fission gas release assumes that power and temperature have remained constant for several half-lives. The fractional release is a function of time and fuel temperature and burnup, and is also dependent on half-life. The release fraction for isotope i is 


$$
\begin{aligned}
F_{1}=\frac{3}{1-\exp \left(-\mu_{i} \tau_{i}\right)} & {\left[\frac { 1 } { \sqrt { \mu _ { i } } } \left[\operatorname{erf}\left(\sqrt{\mu_{1} \tau_{i}}\right)-2 \sqrt{\left.\mu_{i} \tau_{i} / \pi \exp \left(-\mu_{1} \tau_{1}\right)\right]}\right.\right.} \\
& \left.-\frac{1-\left(1+\mu_{i} \tau_{i}\right) \exp \left(-\mu_{i} \tau_{i}\right)}{\mu_{i}}\right] \quad \text { for } \tau_{i} \leq 0.1
\end{aligned}
$$

and

$$
\begin{array}{r}
F_{i}=\left[3 \frac{1}{\sqrt{\mu_{i}}} \operatorname{coth}\left(\sqrt{\mu_{i}}\right)-\frac{1}{\mu_{i}}\right]-\frac{6 \mu_{i}}{\exp \left(\mu_{i} \tau_{i}\right)-1}\left(\sum_{i=1}^{3} \frac{1-\exp \left(-n^{2} \pi^{2} \tau_{i}\right)}{n^{2} \pi^{2}\left(n^{2} \pi^{2}+\mu_{i}\right)}\right) \\
\text { for } \tau_{i}>0.1
\end{array}
$$

with

$$
\begin{aligned}
& \mu_{1}=\lambda_{i} / D^{\prime} \\
& \tau_{1}=D^{\prime} t
\end{aligned}
$$

where

$$
\begin{aligned}
\lambda_{i} & =\text { decay constant for isotope } i(1 / s) \\
t & =\text { total accumulated irradiation time (s) }
\end{aligned}
$$



D. $=\left[\left(0_{0} / a^{2}\right) \exp (-0 / R T)\right] \times 100^{(B u / 2800)}$
erf = error function
Bu = total accumulated burnup (MWd/MTU)
I = temperature $(K)$ for previous four half-lives.

The low temperature radioactive fission gas release for isotope i is defined by

$F_{i}=\left(1 / \lambda_{i}\right)\left[1 \times 10^{-1} \sqrt{\lambda_{1}}+2 \times 10^{-12} \mathrm{P}\right]$

where

$$
\begin{aligned}
& P \quad=\text { specific porer (MWd/MTU) } \\
& \lambda_{1} \quad=\text { decay constant for isotope } \quad(1 / 5) .
\end{aligned}
$$

2.5.3.2 Beyer-Hann Gas Release Model--The Beyer-Hann gas release model is an empirical correlation based on carefully selected data sets wherein maximum fuel temperatures were measured or could be estimated. Reference 28 discusses the development of the basic model, which identifies discreet constant release rates from three different temperature zones in the fuel. The temperature boundaries of these zones and their assigned release rates are shown in Table 3. The release rates were assioned from a statistical correlation of results from seven well-qualified irradiation tests, plus agreement with microcoring results on irradiated fuel pellets. 
TABLE 3. ASSIGNED TEMPRATURE BCUNDARIES AND RELEASE FRACTICNS

\begin{tabular}{lll}
\hline Region & $\frac{\text { Temperature Bounds }(k)}{1473 \text { to } 1673}$ & Release Fraction \\
1 & 1673 to 1973 & 0.141 \\
3 & 1973 to 3073 & 0.807 \\
\hline
\end{tabular}

In addition to the high-temperature gas release indicated in Table 3, a lon-temperature gas release rate has been adod, which has been simplified from the original formulation in Reference 29. The low temperature release rate is a simple linear function of tuel burnup, reaching a maximum of $1.0 \%$ at 20,000 Mwalmiu.

Finally, the NRC-recommended gas release enhancement factor for high burnup fuel (greater than 20,000 Midmiu) has ceen added as an option. This correlation takes the form

$\mathrm{F}_{\text {corr }}=\mathrm{F}_{\mathrm{old}}+\left(1-\mathrm{F}_{\mathrm{old}}\right) \mathrm{Y}$

(211)

where

$$
F_{\text {old }}=\text { uncorrected fractional release rate }
$$


$F_{\text {corr }}=$ corrected fractional release rate.

The function $Y$ has the form

$Y=\frac{1-\exp A(B u-20,000)}{1+B / F_{\text {old }} \exp C(B u-20,000)}$

where $A, B$, and $C$ are constants and have the values

$\begin{aligned} A & =4.36 \times 10^{-5} \\ B & =0.665 \\ C & =1.107 \times 10^{-5} .\end{aligned}$

A convention ceveloped by Soulhier and Notley 32 is used in the application of the Beyer-Hann model. As burnup proceeds, the identity of the time step in which the last highest release fraction was attained must be maintained. If the current release fraction is greater than the last highest value, then the total current inventory is released at the current (all-time high) rate. If this is not the case, then oniy the inventory produced since the attainment of the last higher release value is avaibalefor release.

2.5.3.3 MacDonald-Keisman Gas Release Model--The MacDonaldWeisman fission gas release model considers the release determined by escape of gas from the fuel matrix and release of trapped gas from grain boundaries or dislocations. The model presented in Reference 19 is repeated here. If $k^{1}$ represents the portion of 
fission gas that escapes without being trapped, then

$\mathrm{dn}_{i}=\mathrm{k}^{1} \mathrm{pdt}$

where

$$
\begin{aligned}
d n_{i} & =\text { gas released oirectly in time dt (moles) } \\
d t & =\text { the time increment (s) } \\
p \quad & \text { the gas production rate (moles } / \mathrm{s}) .
\end{aligned}
$$

If the probability of trapped particle release per unit time is $k$, and the number of moles trapped is $C$, then the trapped moles released in dt is $\mathrm{dn}_{2}=k \mathrm{c} d t$. Only a fraction, ${ }^{1}$, of the gas released from traps reaches the surface, thus the total gas released is $a n=k^{1} k c d t+k^{1} p d t$.

If $C$ is replaceo by $(p t-n)$, and integration is performed, one obtains

$n=p t=\frac{1-k^{1}}{k^{1} k}\left[1-\exp \left(k^{1} k t\right)\right]$

At constant power, the total fractional release is

$F=n /(p t)=1-\left(1-k^{1}\right) \frac{1-\exp (-k t)}{K t}$

where $k=k^{l} k$. The constants, $k$ and $k$, have been evaluated from 
data as functions of fuel temperature and density in the form

$A \exp (-B / T-C d+D)$

(216)

where

$T=$ fuel temperature $(k)$

d $\quad$ fuel density (percent of theoretical density)

and for $k$ and $k^{1}$, respectively,
$A=0.25,1.0$
$B=11894.0,6916.7$
$c=0.0,0.333$
$D=0.0,33.95$.

The preceding formulation is extended to variatle power time nistories by assuming reactor operation is described by a series of constant power steps. The number of moles released, $n$, during the $i-t h$ interval is then 


$$
\begin{aligned}
\Delta n_{i}= & n_{i}-n_{1-1}=P_{i}\left\{\Delta t_{1}-\frac{1-k_{i}^{1}}{k_{i}}\left[1-\exp \left(-k_{i} \Delta t_{i}\right)\right]\right\} \\
& +C_{1-1}\left[1-\exp \left(-K_{i} \Delta t_{i}\right)\right]
\end{aligned}
$$

The first teris represent the release during $t$ had the initial concentration been zero. The last term is addional release due to previcusly produced gas. Since the total release from time zero is $n$, the fraction of total gas produced which is released is

$$
F=\left(\sum_{i=1}^{m} \Delta n_{i}\right) /\left(\sum_{i=1}^{m} p_{1} \Delta t_{1}\right)
$$

This fraction is used along with the total gas production isee Section $2,5.2)$ to obtain the gas inventory.

2.5.3.4 GRASS and FAST-GRASS GaS Release Models--GRASS is a highly mechanistic gas release model which accounts for bubble formation, migration, coalescence, channeling and eventual release. The model was developed at Argonne National Laboratory (ANL) and is cescribed in documentation provideo by ANL. 31 FAST/ GRASS is an abbreviated version of GRASS which was designed to run more efficiently.

2.5.4 Nitrogen Release. The release of nitrogen initially present in fuel material fron fatrication occurs as a result of a diffusion transport mechanism. The model proposed by Booth ${ }^{33}$ is used, given 
the following assumptions:

1. The initial concentration of diffusing substance, $C$, is uniform throughout a sphere of radius, a.

2. Transport of material does not occur from the external phase (gaseous nitrogen) back into the initial carrier medium.

The governing equation is

$r \frac{\partial C}{\partial t}=D \frac{\partial^{2}(C r)}{{ }^{\partial}{ }^{2}}$

where
$r$
$=$ radial location (m)
C
= concentration of diffusing substance
t $=$ time (s)
D = diffusion coefficient
C
$=0.0$ when $r=a$

with 
$c=c$ when $t=0$.

By applying a series solution method, the fractional release of the diffusing substance (nitrogen) can be approximated based on the value of $b$

$\mathrm{B}=\pi^{2} \mathrm{D}_{\mathrm{N}_{2}}(\mathrm{~T}) \mathrm{t}$

where

$$
\mathrm{D}_{2}(T)=\text { temperature dependent diffusion coefficient for }
$$$$
t=\text { time from the start of diffusion (s). }
$$

Then, when $B>1$, the fraction of nitrogen released as of time, $t$, equals

$$
\begin{aligned}
& \mathrm{F}_{\mathrm{N}_{2}}=1-6 \exp \left(-\mathrm{B} / \pi^{2}\right) \\
& \text { and, when } B \leq 1 \\
& \mathrm{~F}_{\mathrm{N}_{2}}=6\left[\mathrm{D}_{\mathrm{N}_{2}}(\mathrm{~T}) \mathrm{t} / \pi\right]^{0.5}-3 \mathrm{D}_{\mathrm{N}_{2}}(\mathrm{~T}) \mathrm{t} . \\
& \text { From the experimental data of Ferrari } 34,35 \\
& \mathrm{D}_{\mathrm{N}_{2}}(\mathrm{t})=\left(1 \times 10^{-12}\right) \exp (\mathrm{G})
\end{aligned}
$$


where

$G=20202.0\left(\frac{1.0}{1673.0}-\frac{1.0}{T}\right)$

(224)

2.5.5 Fuel Rod Void Volumes. Void volumes computed by FRAPCON-2 include the pellet dishing, the fuel-cladding gap, the volume crack, the plenum, the open porosity, and the roughness volume. These are calculated as indicated below.

2.5.3.1 Pellet Dish volume--The volume between pellets is calculated and included as part of the overall volume in the internal gas pressure model. The interpellet volume is calculated at each time step as the difference between the cold-peliet and hotpellet geometries.

Figure 34 shons (a) a cold-pellet interface configuration and (b) an exaggeratea hot-pellet interface configuration. The void volume available for internal fill gas is defined by the cross hatched areas ( $A$ and $B$ in the figure).

2.5.5.2 Fuel-Cladding Gap Volume--The fuel-cladding gap volume is calculated by considering the area between two concentric cylinders. The outer cylinder is assumed to have a diameter equal to the diameter of the clading inside surface based on plastic deformation. The inside cylinder is assumed to have a dimeter equal to the diameter of the relocated fuel pellet.

2.5.5.3 Fuel Crack volume--As the fuel expands, extensive 


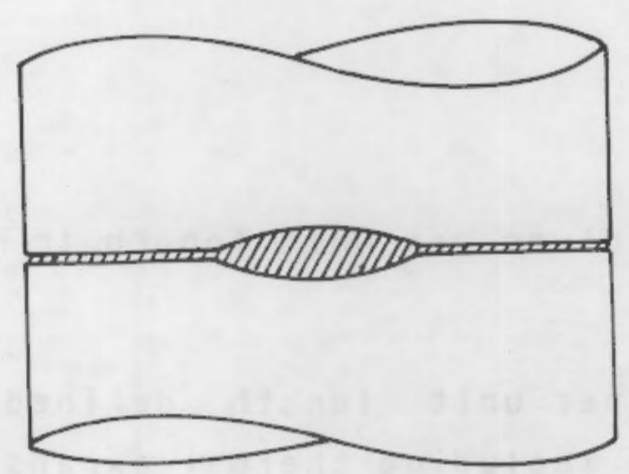

(A) Cold pellet interface

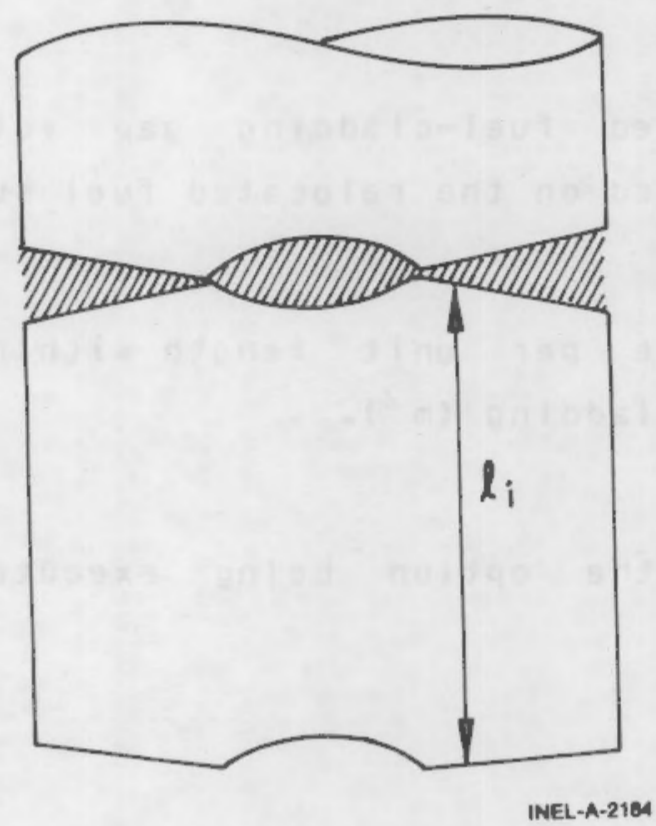

(B) Hot pellet interface

Figure 34. Dish void volume.

cracking occurs due to the righ thermally-induced stresses resulting in a relocated fuel surface. If FRACAS is used, this crack volume is computed as

$\mathrm{v}_{c}=\mathrm{v}_{\mathrm{eg}}-\mathrm{v}_{\mathrm{TX}}-\mathrm{v}_{\mathrm{g}}$ 
where
$v_{c}=$ fuel crack volume per unit length $\left(m^{2}\right)$ radial nodes including thermal expansion, swelling, and densification $\left(\mathrm{m}^{2}\right)$
$V_{\text {eg }}=$ fuel volume per unit length defined by expanded
$V_{\mathrm{TX}}=$ the computed fuel-cladding gap volume per unit length based on the relocated fuel surface $\left(m^{2}\right)$
$v_{g}=$ the volume per unit length within the thermaliy expanoed cladding $\left(m^{2}\right)$.

However, if PELET is the option being executed, the crack volume is calculated as

$\mathrm{v}_{\mathrm{cr}}=\mathrm{v}_{\mathrm{rfs}} \mathrm{VFF} / 100.0$

where
$v_{\text {cr }}=$ fuel crack volume per unit length $\left(m^{2}\right)$
$V_{\text {rfs }}=$ fuel volume (contained within the relocated fuel surface) per unit length (m2)
VFF $=$ void fraction within fuel $(\%)$. 
2.5 .5 .4 Plenum Volune--The plenum volume is calculated from geometry considerations of the thermally expanded cladding and the thermal expansion, censification, and swelling of the fuel. The volume of the hoto-dom spring is considered.

2.5.5.5 upen Porosity volume--A portion of the initial fabrication porosity is open to free gas flow wich is given by the expressions

$$
\begin{aligned}
V_{\text {por }}= & 0.0 \\
V_{\text {por }}= & 1.97 \times 10^{-8}\left(94.0-G_{\text {den }}\right) \text { when } G_{\text {den }} \geq 94.0 \\
v_{\text {por }}= & 2.77 \times 10^{-4}-3.818 G_{\text {den }} \\
& -1.43 \times 10^{-8} G_{\text {den }}^{2}+2.497 \times 10^{-10} G_{\text {den }}^{3} \text { when } G_{\text {den }}<91.25
\end{aligned}
$$

where

$$
\begin{aligned}
& v_{\text {por }}=\text { porosity volume per unit length }\left(\mathrm{m}^{2}\right) \\
& G_{\text {den }}=\text { DEN }-1.23
\end{aligned}
$$$$
\text { DEN = fuel oensity (percent of theoretical density). }
$$

2.5.5.6 Roughness Volume--The roughness of the surface of the fuel and cladoing result in a small vold volume accounted for by 


$$
V_{\text {rough }}=\frac{5.27 \times 10^{-5} \pi D_{\mathrm{P}}}{\mathrm{V}_{\mathrm{f}}}
$$

where

$$
\begin{aligned}
& v_{\text {rough }}=\text { roughness volume per unit length }\left(\mathrm{m}^{2}\right) \\
& v_{\mathrm{p}}=\text { initial pellet diameter }(\mathrm{m}) \\
& v_{\mathrm{f}}=\text { geometric fuel volume per unit length }\left(\mathrm{m}^{2}\right)
\end{aligned}
$$

The gas pressure response resulting from the above models feeds back into the mectianical and temperature response models in the iteration scheme.

2.6 tuel Rod Failure Models

A set of models has been developed for fRAPCON-2 to predict the propability of failure of zircaloy cladding under a variety of steday state and transient conditions. Collectively, these mocels form the FRAIL-3 (LEAP Integrity Limit) package. This package interfaces oniy with the FRACAS-I mechanics option.

Each model has the capability of predicting a different mode of zircaloy tuel rod cladoing failure. Probabilities of failure are calculated for each failure mode, then appropriately combineo to 
yielo a net probability of failure for the fuel rod cladoing. The faiture models considered by FRAll-5 are cladding melt, eutectic melt, excess oxide, excess ballooning, cladding collapse, overstress, crack growth, overstrain, fatigue, stress-rupture and flow blockage. Not all of the fallure models in Frall-s are applicacle to steaoy state. Unly the steady state failure models are discussed belom.

2.0.1 Model for Cladcing Melt. The cladding melt rodel predicts cladding failure whenever the MATPRC meft temperature or a mett temperature supplicd by the user is exceeded. The probability for fallure is set to one when this condition exists.

2.6.2 Model for Eutectic Melt. The clading eutectic melt model predicts cladding failure when (TCOOL/2 + TCLAD)/1.5>1233 K where TCUJl is the coclant temperature and TCLAL is the average clad temperature $(K)$. The probability for failure is set to one when this condition exists.

2.6.3 Model for Excess oxide. If the thickness of the clading oxide layer is greater than $17 \%$ of the original cladding wall thickness, failure of the cladding is assumed to occur. If the oxide layer thickness is less than 17\% of the original wall thickness, the probatility for fallure is assumed to be zero.

2.6.4 Model for Uverstress. The assumptions in the overstress failure model are:

1. Uverstress failure must be madeled using true hoop stress. 
2. The true hoop stress at failure is a function of temperature, cold mork and fast neutron fluence.

3. The cladoing cross section is circular, although the inside and outside surfaces are not necessarily concentric.

4. The cladoing axial radius of curvature is infinite.

5. Wall thinning is the primary deformatin that causes an an increase in true stress.

6. The cladoing is inconpressible.

The overstress model uses the cladoing mechanical limits model described in Reference 3. This model uses a correlation between circumferential terperature variation for which the overstress model assumes a $50 \mathrm{~K}$ variationl and circumferential strain to compute local wall thinning. The local wall thinning is then used to convert the true burst stress to an effective true burst stress for idealized symmetric deformation. The overstress model compares this with the effective true hoop stress from the current time step which is computed by

$\bar{\sigma}_{\theta}=\operatorname{HSTRESS} \star(1+$ HSTRAN $) /(1+$ STRANR $)$

(231) 
where

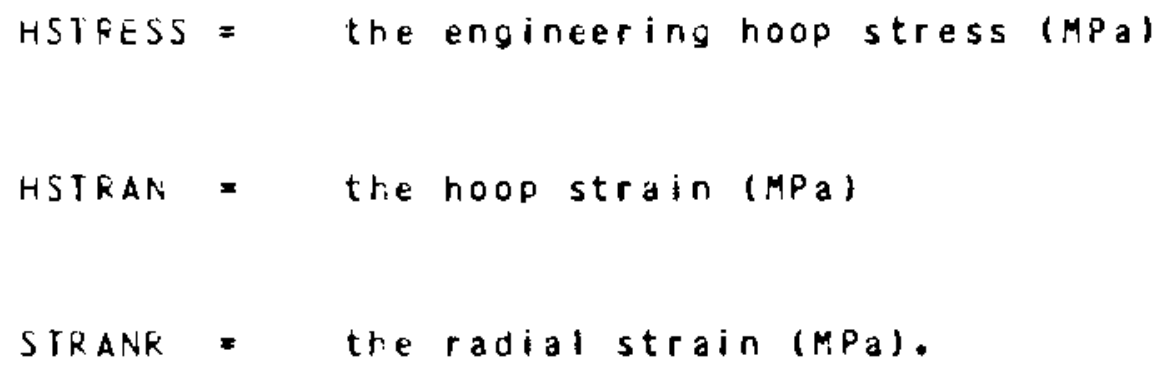

For the purpose of the FRAIL-5 subcode, the protablltiy of failure as a function of stress ano temperature is needed. Tris is obtained by assuming a distribution of failure stress about the mean failure stress.

The beta cistribution function was found to be most representative of the failure stress data. The beta distritution is Iimited to a tinite interval and may be adjuted to include all available data. for a given temperature, values of stress mhich lie above the defineo interval result in failure (probablity of one), while values which fall below the interval result in no failure (probatility of zero). Because the beta distribution is defined only on the interval 0 to 1 , it is necessary to normalize both the failure stress and the standard deviation of the failure stress to this interval. By a simple variable tranformation, the normalized failure stress is found from the expression

$\bar{x}=\frac{\vec{\sigma}_{\theta F}-B}{T-B}$ 
where

$$
\bar{x}=\text { rormialized failure stress (MPa), }
$$

and $E$ and $T$ define the interval of avaitable failure stress data. This interval mas chosen to be three standard deviations above and tho stanoard oeviations delow the mean failure stress, so that for a given temperature, the available test data are includec in the interval.

The normalizeo standara deviation is found from the equation

$s=s^{\prime} \frac{\partial \bar{x}}{\partial \bar{\sigma}_{G F}}=\frac{s^{\prime}}{T-B}$

where

$$
\begin{aligned}
s^{\prime} & =\text { ncrmalized standard aeviation; that ls, stanoara } \\
& \text { deviation of } x \\
= & \text { standard deviation of } \bar{\sigma}_{\theta F} .
\end{aligned}
$$

The cumulative beta distribution, ottained by integration of the frequency oistritution tunction, is used to determine the probability of failure as a function of stress and temperature.

2.6.5 Model tor Crack Growth. The well-known phenomenon of stress corrosion crackinc (SCC) is an important contributor to stress 
rupture and is considered in this failure model. A literature search for quantitative cata explaining the SCC phenomenon has resulted in only tro data sets wich can be used in model development. 37,38 All other literature has of a qualitative nature. Two quantitative papers used a fracture mechanics approach to explain the SCC phenomenon. This section oriefly explains the fracture mechanics approach. Adaptations were made to the rethod to assure compatatility hith FRAL-5. The adaptations are also presented.

References 36 and 37 state that the SCC phenomenon can be described by the crack growth equation

$$
\frac{\mathrm{da}}{\mathrm{dt}}=\mathrm{CK}_{\mathrm{I}}^{4}
$$

where

$\begin{aligned} K_{I} & =\text { stress intensity factor } \\ c & =\text { material constant }(\mathrm{m} / \mathrm{s}) \\ \mathrm{a} & =\operatorname{crack} \text { length (m) } \\ \mathrm{t} & =\operatorname{time}(\mathrm{s}) .\end{aligned}$

Here, the crack length is measured through the cladoing wall. The axial and tangential components of crack length are not considered by Equation $(234)$. 
The stress intensity factor, $K$, may be toung from the equation

$K_{I}=\sigma_{0} Y \sqrt{a}$

where

$\begin{aligned} \sigma_{\theta} & =\text { appliea tensile hoop stress (MPa) } \\ r \quad & =\text { fracture mechanics flaw geometry factor. }\end{aligned}$

The material constant, $C$, in Equation (234) was found to be dependent on temperature and iodine concentration. The experimentally aeterminea correlation for $c$ was found to be

$\mathrm{C}=1.32 \times 10^{-7} \mathrm{I}_{2}^{3 / 4} \exp \left(\frac{-35900}{\mathrm{RT}}\right)$

where

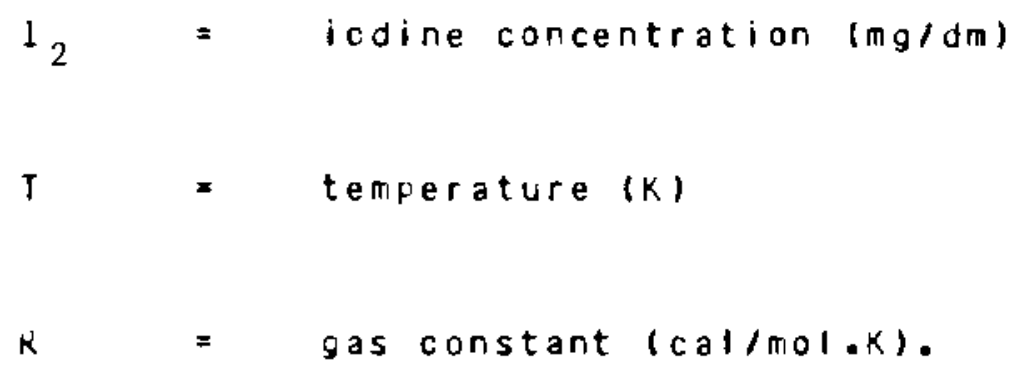

For a given temperature and iodine concentration there is a critical stress intensity factor, $K_{\text {ISCC }}$ below which SCC will not occur. This implies that there exists a threshold stress below 
which SCC will not occur. This threshola stress is given by

$\sigma_{\text {threshold }}=\frac{K_{\text {ISCC }}}{Y \sqrt{a}}$

This threshold stress may also be found from the following empirical relationship

$\sigma_{\text {threshold }}=\frac{(2710-3 \mathrm{~T}) \mathrm{I}_{2}^{-3 / 16}}{\mathrm{Y} \sqrt{\mathrm{a}}}$

where all variables are as previously defined.

knowing the crack velocity given by Equation (234), the crack length can be determined at any foint in time. This crack length may then be compared to the critical cracklength in order to determine if the fuel rod has failed. The critical crack length is defined as the critical depth necessary to cause instantaneous failure by stress rupture.

All variables necessary lo calculate the crack length are made available to FhAIL-5 frun. MATPRO and the FRAPCON-2 code, with the exception of a, the initial crack depth, and $y$, the flaw geometry factor. Until a more exact determination of these variables can be made, the following values are assumed. The initial flaw in the cladding is assumed to be a pit-shaped flaw, $12.7 \mathrm{~mm}$ in depth. For pits, $Y=1.29$. These values of $Y$ and a were found most frequently in the lot of stress-relieved twbing used in the experiments describeo in References 36 and 37 . 
The critical crack length may be found, as suggested in References 36 and 37 , from the expression

$$
\frac{a c}{w}=1-\frac{\sigma}{\sigma_{B}}
$$

where

$$
\begin{aligned}
a_{c} & =\text { critical crack length }(m) \\
& =\text { cladjing thickness }(m) \\
& =\text { applied hoop stress (MPa) } \\
\sigma_{B} & =\text { burst strength (MPa). }
\end{aligned}
$$

Because the frequency distribution function of the burst strength is known, the frequency distribution of $a_{c} / w$ may be calculated. By knowing the probability frequency distribution of ac' it is possitte to ottain a probability of failure due to SCC from a knowledge of the calculated crack length.

2.0.6 Model for Lverstrain. The assumptions used in the overstrain failure model are

1. The mean failure strain can be correlated with temperature by least-squares fitting to the fallure strain oata base.

2. The distribution of the failure strain about the mean 
failure strain can be approximated by a beta probability cistribution.

3. The failure strain is not a function of the hydrogen, oxyoen, cesium, or iodine content of the cladding.

The overstrain failure model calculates the probability of failure as a function of strain and temperature. The strain at failure is assumed to be distributed according to the beta distritution. The upper and lower linits are set at +2 ano -2 standarc deviations, respectively, from the mean failure strain. The standaro oeviation is $16 \%$ of the mean failure strain.

The mean failure strain as a function of temperature is obtained from the cladoing strain at rufture model described in Referelce 37. The effects of cold work and irradiation level are taken in to account

2.7 Uncertainty Analysis Option

An uncertainty analysis option has been developed for FRAPCON-2 so that a user may easily obtain estimates of the uncertainty in calculated code outputs. The option has specifically been designed so that the user may perform an uncertainty analysis on a FRAPCON-2 case in a understanable and systematic manner. The option further provides for a sequential development of analysis complexity by allowing the user to restart and continue an analysis from intermediate points. One gaal of the option is to provide to all users a straightforward technique based on sound methodology for estimating code uncertainties. 
2.7.1 Uncertainty rethodology. The uncertainty analysis option is based on the response surface method. Any of the output variables of a computer code may te termed a response. There is some functional relationship between a response and the input varlables. In the space of the input variables, this relationship cefines a surface, and nence the term response surface. When the code is ratner simple, this surface may be determined analytically over the entire range of the input values. More often, as in the case of FRAPCON-2, the surface may be known only through the code, as the ranje of inputs ano problem types is very large. Thus, the complete true response surface cannot be determined analytically. The response surface method of uncertainty analysis is based on a systematic sampling of the true surface which is then approximated by a potynomial equation in the independent (input) variables. In effect, the true surface is approximated by a smooth surface. ${ }^{39}$

The polynomial equation approximating the true surface is derived as follons. Let $y\left(x_{i}\right.$, denote the code response as a function of $x_{i}=x_{1}, x_{2}, \cdots, x_{K}$ inputs. The Taylors serles expansion about any point is then given by

$$
\begin{aligned}
Y\left(x_{i}\right)= & Y\left(\mu_{i}\right)+\sum_{i+1}^{K} \frac{\partial Y\left(\mu_{i}\right)}{\partial x_{i}}\left(x_{i}-\mu_{i}\right)+1 / 2 \sum_{i=1}^{K} \frac{\partial^{2} Y\left(\mu_{i}\right)}{\partial{ }_{i}^{2}}\left(x_{i}-\mu_{i}\right)^{2} \\
& +\sum_{\substack{i, j \\
i<j}}^{K} \frac{\partial^{2} Y\left(\mu_{i}\right)}{\partial x_{i} \partial x_{j}}\left(x_{i}-\mu_{i}\right)\left(x_{j}-\mu_{j}\right)+\text { higher order terms }
\end{aligned}
$$

Truncating the Taylors series at second order terms, the desirea polynomial equation is obtained by identifying the coefficients of the polynomial with the partial derivatives of the series expansion. The coefficients are estimated from sample values 
of the true response surface obtained by perturbing the nominal inputs. For a seconc order polynomial to reasonably approximate the true surface, the region of the surface being sampled must be small enough so that larye irregularities are not present. Experience has shown that a range of plus and minus one standard deviation $( \pm 1 \sigma)$ in the input variable uncertainties will usually satisfy this requirement for FRAPCEN-2.

The polynomial approximation to the true response surface may be usec to examine the behavior of the true surface in the region of the sample space without the buraen of excessive cost. In particular, the polyrionial can be used to study the propagation of errors through trie code and their effect on the uncertainty in computed outputs. Thus, an estimate of response uncertainty arid the relative contributions of input variables to this certainty may be obtained using the response surface method.

Dnce the user has selected a base case problem and made a cnoice of output responses and input variables, the folfowing procedures will te followed by the code to obtain the desiced final results, the estimates of response uncertainties.

1. An experimental design will be chosen. Inls is simply a pattern for ferturting the independent varlables of the case. The pattern is abtained in matrix format where the columns correspond to inputs and the rows correspond to the individual analysis that must be performed. The case is run as nany times as the design dictates, each time varying the input variable perturbations according to the pattern. 
2. The response surface equations are then generated at each FRAPCON-2 time step using the information cerived from step one. Basically, a multiple regression routine useo with certain simplifications arising from the orthogonal properties of the experimental design.

3. The resfonse surface equations are used to generate uncertainty distritutions for the response parameters. Second oroer error propagation analysis is usec to estimate the means anc variances of the responses.

4. Finally, estimates of the fractional contributions to the response variances are made to indicate the relative importance of individual input rariables.

2.7.2 Uncertainty Application. The uncertainty analysis option in FRAPCON-2 is essentially ioentical to that in FRAP-T5 40 . The only difference lies in expanded input and response lists that reflect the oifferences in the two codes. The user is referred to Reference 41 for detailed discussions of the development and assessment of the method. The purpose of this section is to suggest possible use of the option and specitic limitations of the methoo of which the user shoula be aware.

Obviousiy, the most important feature of the option is the ability to determine the uncertainty in code outputs that result from propagating uncertainties in inputs through the cooe. Equally important for code development, however, is the ability to determine the relative contributions to the overall uncertainty of each input variable. This can lead to studies on the need for specific code development or experimental programs. For example, if it is found 
that fuel ther ${ }^{2}$ al conductivity uncertainty contributes $80 \%$ of the uncertaincy in clading temperature but fuel Poisscn's ratio contributes less than $1 \%$, then future rork should be directeo at refining the uncertainty in fuel thermal conductivity.

Another use of the option is a traditional sensitivity analysis. Here the user should be aware that the interpretation of the perturbations that define the experimental desion as one stancaro oeviation is not made until the very last step of the analysis. At that point the interpretation is necessary in order to infer information about the uncertainty distribution of the output responses. If the user is willing to forego this inference, then the perturbations naking up the experimental design can in fact be arbitrary. If, for example, the user wishes to study the sensitivity of a particular response to $10 \%$ deviations in a family of input variables, this analysis is entirely possible. The output coefficients of the response equation can be interpreted as sensitivity coefficients given in units comparable to the responses. The coefficients can be ranked by absolute macnitude and this ranking represents the sensitivity of the response to each input. Thus tracitional sensitivity stucies may easily be executed.

Dccasionally, the user must be prepared to question the validity of results produced by the option. The estimates of response uncertainty are based on approximations (the response surface equationsl to the true code output. The accuracy of these estimates therefore depends airectiy on the degree to which the equations really do approximate the true code output. An often used method to evaluate this is to examine the distribution of residuals formed by taking the differences between the code predictions and the response surface equations. At present, the code fits a full model equation to the data, resulting in very smal, 
residuals. The best metrod is to examine residuals from data not used to fit the equations. This entails generating more computer data. However, the nominal case is always run and only used in equation fitting for quaratic analyses. The user is therefore a drised to compare the nofiral case with the response equations. If the true code outfut is actually linear, the mean will equal the nominal. The difference tetween the two may be taken as some measure of the quality of the linear response equation approximation.

\section{GENEKAL CODE DESCRIPTION}

3.1 Code Structure ana Solution Routine

FRAPCQN-2 is a large ano complex code that contains over 200 subroutines. The hierarchy of subroutines, their function and their interrelation are discussed in detail in Appendix C. This section discusses the code structure, solution scheme and the major subroutines involved in the solution scheme.

3.1.1 Code Structure. The FRAPCON-2 sucroutines have been grouped in packages, not all of which need to be compiled for every run. These packages are listed in table 4. Note that every execution requires the FRPCON package and the MATPRO package; the former contains the driver routine, the setup routines, and the thermal models. Using only these tmo packages restricts one to the FRACAS-I mechanlcal modeling option and precludes selection of the GRASS Or FAST-GRASS fission gas release models.

The other packages listed in Table 4 correspond to the models of the same name discussed in Section 2 . 
TABLE 4. MAJCR FRAPCON-2 PACKAGES

Package

Cescription

PRFCCN

The main section of the code, including all of the thermal models; also includes the uncertainty analysis routines, the FRAll cladoing failure model, and the FRACAS-I rechanics rodel.

FRACAS-II Contains the subroutines comprising the FRACAS-1I ceformable pellet mechanics model.

FELET

Contains all of the subroutines that comprise the PELET/RAOIAL mechanics mooel.

AXISYM

Contains the subroutines comprising the detailec finite element mechanics mooel.

MATPRC

The MATPRO material properties packase.

GRASS

Contains the subroutines which comprise the GRASS fission gas release model.

FAST-GRASS

Contains the subroutines which comprise the fast GRASS fission gas release model. 
3.1.2 Solution Scheme. Figure 35 shows a flowchart of FRAPCON-2 beginning with case setup, following through the converoence loops and ending with output. Each major section of this sequence will be aiscussed, togetiner with the subroutines involved. To aid this discussion. Figure 36 presents an aboreviated outione of the main suoprogram, FRPCLA, arranged in the same oroer as the flowchart. Major sutroutines appear in the figure as do the major FORTRAN IOOPS.

The first portion of the flowchart has to do with case setup and initialization. This includes reading the input data, the oynamic dinensioning procedure, initializing variables, and an initial problem cescription output. The subroutines listed in lable 5 are involved in the setup and initialization.

Next, the cooe enters the first of four major loops in the FGRTRAN coding, the Time Step Loop. The Time Step Loop encompases virtually all of the rerainder of the FRAPCON-2 code. In each execution of the Time step loop, the code solves for the thermal and mechanical equilitrium of the fuel rod at a new point along the rod power versus time history input ty the user. Those subroutines which are executed only once per time step are listed in Table $t$.

Three additional loops exist in the code. The next loop encountered within the Time Step loop is the Gas Releae Loop. This loop is cycled until the value for calculatedroointernal oas pressure fopendent on temperature, volume, and fission gas release) converges. Subroutines called from within this loop are listed in Tabie 7 .

The next inner loop in the coding is the Axial Node Loop. For 

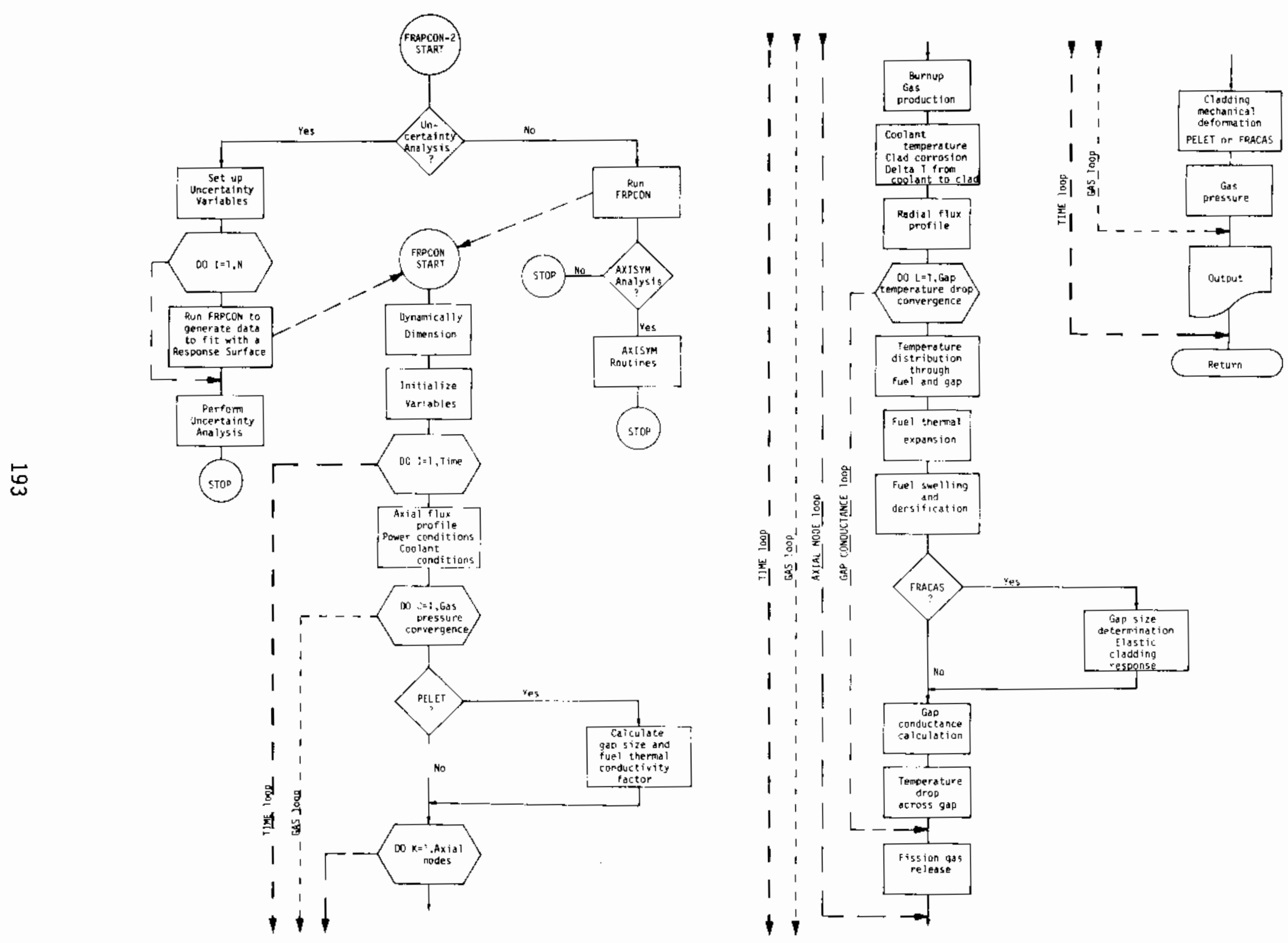

Figure 35. FRAPCON-2 flow chart. 


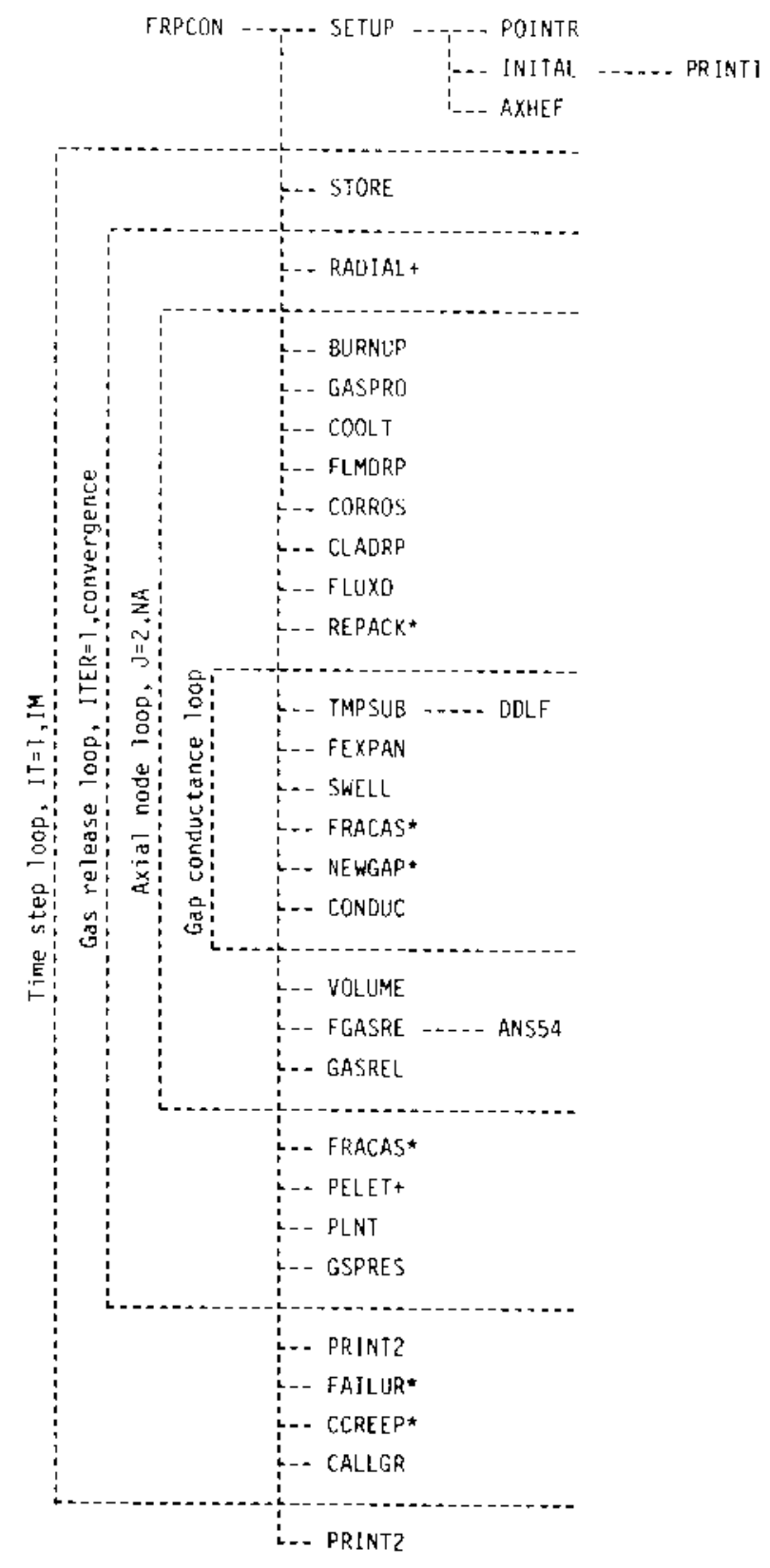

*FRACAS-I or II options only +PELET option only

Figure 36. Calling sequence for FRAPCON-2 subroutines. 
TAELE 5. INITIALIZATION SUBRGUIINES

Subroutine

Description

SETUP

Reads the cata input pertaining to the problen size requirements.

FOINTR

Performs the dynamic dimensioning procedure.

IN I T AL

Reads the remaining problem description input anc initializes the variables.

FKINTI

Gerierates the output reflecting the initial conditions and specifications of the fuet rod anc lists the proposed power history.

$\triangle X H E F$

Calculates the axial power profile as it affects the axial regions of the fuel rod and also any varying axial porer profile changes. 
TAELE 6. SUBrCUTINES IN THE TIHE SteP LCCP

Sutroutine

Description

STCRE

Stores variable values as necessary to account for history aependency.

PR IN T 2

Generates output for the code that preserts converged values for all of the axial nodes for both thermal and mechanical solutions.

FAILUR

A package of sutroutines which calculate the probability of cladoing failure based on an analysis of the FRACAS-I generated cladding conditions.

CCREEP

The cladding creep portion of the fRACAS rechanics mooel.

CALLGR

Calis the GRASS or FAST-GRASS fission gas releast packages. 


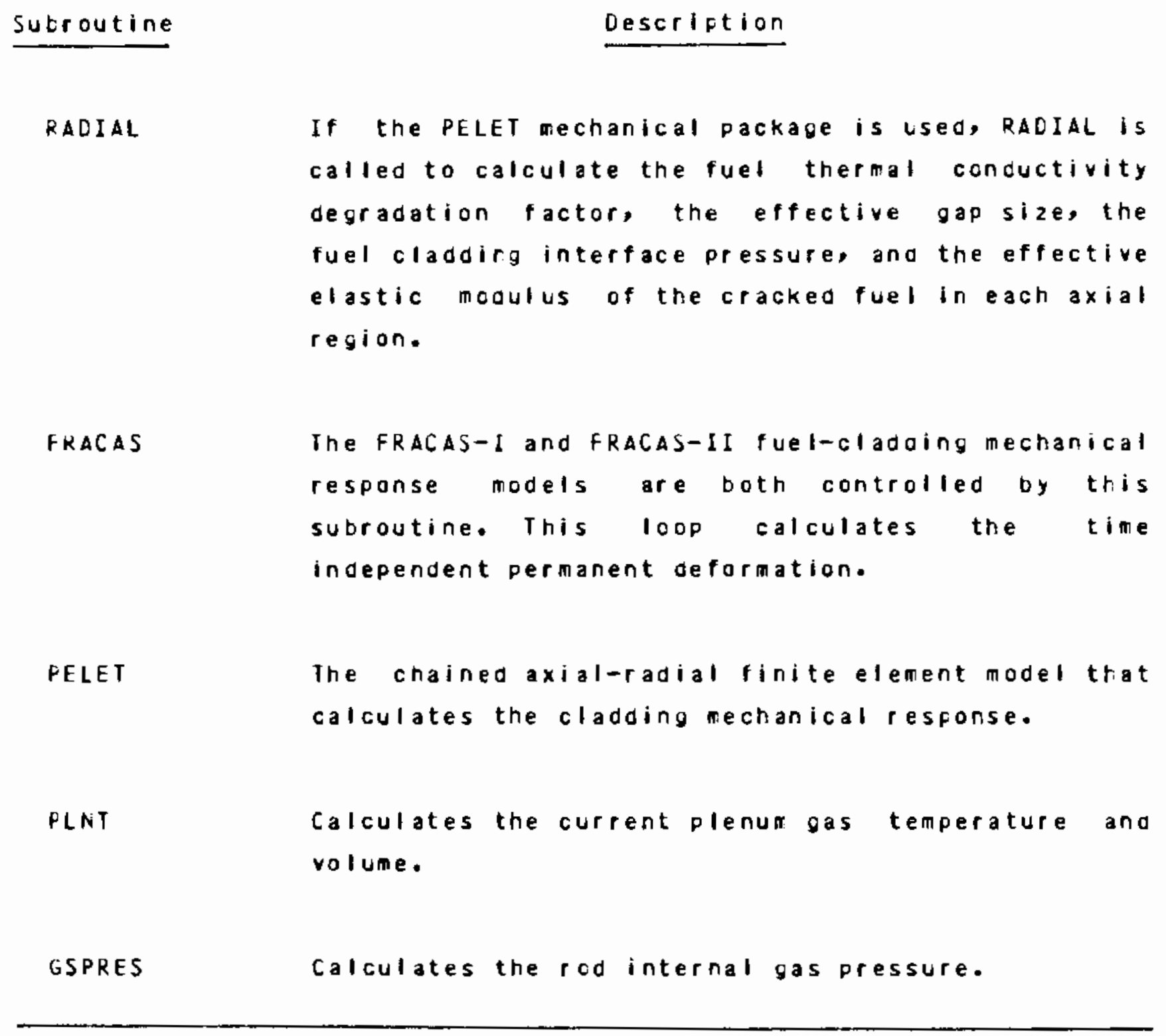


every pass through the Gas Release Loop, the Axial Nooe loop sequences through tach of the axial regions defired by the input. The subroutines controlled by this loop are listed in Table 8.

The innermost loop is the Gap conductance Loop. This loop iterates on each axial node until thermal equilibrium in the radial direction is achieved. Thermat equilibrium is sionifled by a converseo value ror the calculated temperature drop from the fuel outer surface to the cladding inner surface. The subroutines listed in Table 9 comprise the Gap Conductance Loop.

At the completion of all the time steps, and before returning to the ariver packase, a final call to PRINT2 is made. Inis call results in the printing of a summary table for the entire power history of the rod.

3.2 Code Results

FRAPEON-2 generates fuel rod response information as a function of fuel rod fabrication informationg boundary conditions, and power nistory. This information is provided to the user in the form of printed output ano in the form of plots (optionall. The capability also exists to supply this information for steady state initialization of the FRAP-I5 or FRAP-T6 computer codes. The information provioed to the transient fuel rod analysis code consists of permanent burnup effects such as cladding creepoown, fuel skelling, fuel oensification and fission gas inventory. This section presents the important response parameters, the plotting packave and information on the FRAPCON link with FRAP-T. 


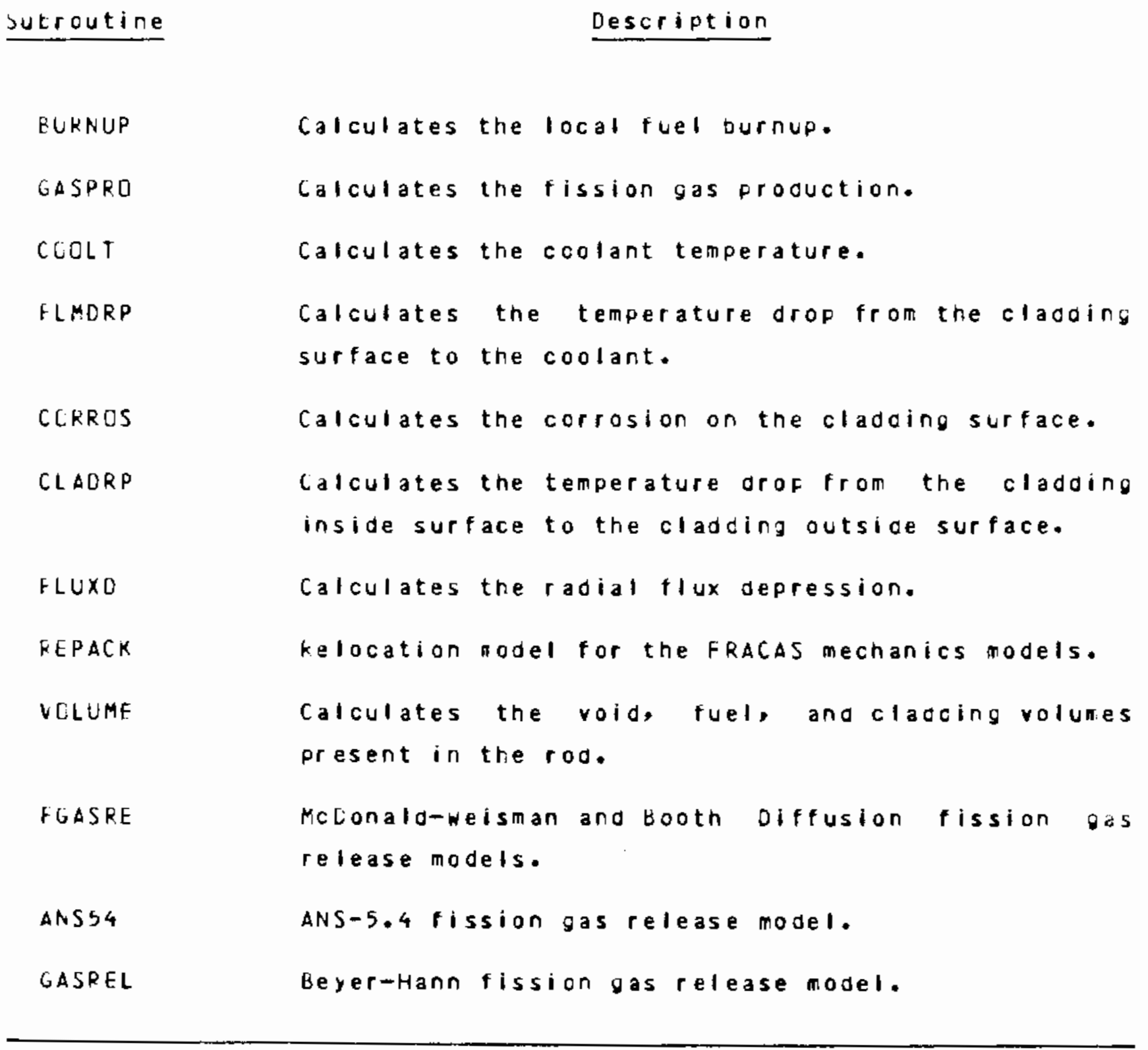


taEle 9. SubrCutines in the gap cCNOUCtance lcop

sutroutine

Description

TMPSUB

Calculates the radial tenperature distribution through the fuel.

CDLF

Solves the equations for the radial heat balance.

FEXPAN

Fuel thermal expansion routine.

SWELL

Calculates fuel swelling and fuel censification.

FRACAS

If the fRACAS rechanics models are used, this subroutine calculates the new position of the clading due to deflection caused by internal oas pressure changes.

NEWGAP

calculates the new fuel-clading gap size lused with the FRACAS mechanics models only).

CCNDUC

Calculates new values for the gap conductance anc the fuel-clading sap temperature drop. 
3.2.1 Fuel Rod Response. rRAPCON-2 provides the calculated fuel rod thermal, mechanical and pressure response data. Ine results are presentes in tnree forms: an axial rejion printout, a powertime step printout, and a sumary paoe printout.

The axial rejion printout presents local information on power, time, time step and burnup. Also presented are rod radial temperature distrioution, coolant temperature, cladding stresses and strains (both recoverable and permanent), gap conductance, fuelcladding interfacial pressure, and coolant film neat transfer information.

The power-time step printout presents roo burnup, void volumes and associated temperatures, mole fractions of constituent vases and release fractions, total moles of rodgas, and rod gas pressure. Also this printout presents stresses, strains, temperatures and stored energy as a function of axial region.

The sumary page printout presents time-dependent information about the hot axial rejion. This includes temperatures of the cladjing, fuel-clasiong sap, and fuel; fuel-cladding interfacial pressure; cladding stress and strain; fuel outside diameter; gap conductance and gas pressure; zircaloy oxide thickness; and hydrogen uptake.

3.2.2 Plot Packaje. Tne FRAPCON-2 plotting package is nade up of suoroutines which nake use of the IGS graphics systen. Tne plot information is stored on unit TAPEl? Oy FRAPCUN-2 and is processed Dy the plot package wnich generates the curve information on unit IAPE 10. TAPEL can be used to generate the plots on computer system devices such as a FR80 microficne plotter, a microfilm plotter, and 
3.2.3 FRAP-T Initialization. FRAPCON-2 contains subroutine RESTFS which, when the fiog NTAPE is set to 1 , stores sets of history depenaent inforkation for each power-time step. This information is storec on unit tAPEl ano is for FRAP-T initialization. This gives the user the ability to model the fuel rod response of a rod which experiences significant burnup prior to some transient excursion.

3.3 FGatures of FRAFCON-2

FKAPCCid-2 has been designed with special features to aid the user. The code has been dynamically dimensioned so that the code may be implemented on computers with limited core storage. The code also nas a restart feature which allows for modeling power histories which require more than loc power-time pairs the maximum number of porer-time pairs wich can be input for any single run is limiteo to 1001 .

3.3.1 Dynatic Dimensioning. FRAPCON-2 has been oynamically dimensioned so that a minimum amount of core storage is required for any given fuel modeling process. Those parameters which are a function of the problem size are dimensioned to the exact size required by the axial and radial nocalization and the number of power-time steps. The user can set the core size based on the number of axial and radial nodes and the number of time steps.

3.3.2 FRAPCON-2 Restart. In the event that a user requires more than 100 power-time steps for the analysis of the behavior of a fuel rod, the capadility exists to restart the code. This allows the 
user the freedor: to model the steaoy state behavior of a fuel rod in as much oetail as is aeemed necessary. When NRESTR is set to 1 , FRAPCON-2 stores information for a FRAPCON-2 restart on unit TAPE2. This information can then be used to continue an analysis which requires more than lco poner-time pairs. The restart option is not drailabte when the PELET mechanical modeling option is chosen. 


\section{KEFERENCES}

1. EGés Ioaho Inc., Quarterly Technical Progress Report on hater keactor Safety Programs Sponsored by the Nuclear Regulatory Commission's Livision of Reactor Safety Research, OctoberCecember 1978, NUREG/CK-0512, TREE-1298, January 1979, p.43.*

2. EGEG Idaho Inc., Guarterly Technical Progress Report on water Reactor Safety Programs Sponsored by the Nuclear Regulatory commission's Division of Reactor Safety Research, January-March 1980, NUREG/CR-1400, EGG-2031, April 1980, pp. 19-20.*

3. L. L. Hagrman et al., MATPRC-Version 11 (kevision I): A Handbook of raterials properties for use in the analysis of Light water feactor Fuel Rod gehavior, NUREG/CR-0497, TFEE-1280, Rev I, February 1980.*

4. D. R. Coleman et al., FKAP-53: A Computer Code for the Steady state Analysis of Oxide Fuel Rods - Model Assessment Report, NUKEG/CR-0786, TREE-1352, R4, APRil 1979.*

5. D. D. Lanning et al., GAPCEN-THERMAL-3 Code description, PNL2434 , January 1978.

- EGEG Idaho, Inc., Quarterly Technical Progress Report on water Reactor Safety Programs Sponsored by the Nuclear Regulatory Comission's Division of Reactor Safety Research, July-September 1978, NUREG/CR-0412, TREE-1294, October 1978, pp. 45-49.** 
7. M. P. Bohn, FKACAS: A Subcode for the Analysis of Fuel PetletCladding Mechanical Interaction, TREE-NUREG-1028, April 1977.

8. B. B. Mikic, Thermal Contact Conductance: Theoretical Considerations, International Journal of Heat Mass Transfer, yolume 14, pp. 205-214.

9. K. E. Willford et al., The Analysis of Fuel Relocation for the NRC/PNL Halden ASsemblies IFA-431, IFA-432, and IFA-513, NUREGI CR-0588, PNL-2709, APriI 1980*

10. C. K. Hann et al., Data Report for the NRC/PNL Halden Assembly IFA-432, NUREG/CR-0560, PNL-2673, 1978. **

11. D. D. Lanning and M. E. Cunningham, Startup Data Report for NRC/ PNL Halden Assembly IFA-513, NUREG/CR-0862, PNL-2948, July 1979.*

12. M. E. Clinningham, D. D. Lanning, and S. D. Montgomery, A Procedure for the oualitative Interpretation of Fuel Centerline Thepmocouple kesponse to step Poner Decreases, NUREG/CR-1012, PNL-3090, Octoter 1979.*

13. F, Dittus and L. M. K. Boelter, Heat Transfer in Automobile Radiators of the Tubular Type, university of California Publications in Engineering, 2, 13, 1930 pp. 443-461.

14. W. H. Jens and P. A. Lottes, Analysis of Heat Transfer, Burnout, Pressure orop, ana Density Data for High-pressure Water, ANL$4627,1951$. 
15. F. Kreith, Principles of Heat Transfer, oth Edition, Scranton: International Eook Company (1964).

16. C. E. beyer et al., GAPCON-THERMAL-2: A Computer Program for Catculating the Thermal Behavior of an oxide fuel Rod, BNiL1098 , November 1975.

17. A. M. Ross and $F$. L. Stoute, Heat Transfer Coefficients Between $\mathrm{UD}_{2}$ and Zircaloy-2, CRFD-1075, AECL-1552, June 1962.

18. A. C. Rapier, 1. M. Jones, and J. E. McIntosh, The Thermal Conductance of Uranium vioxide/stainless steel Interfaces, International Journal of Heat Mass Transfer, volume 6 , pp. 397$416,1963$.

19. N. Todreas ano G. Jacobs, Inermal Contact Conduction in Reactor Huel Elements, Nuclear Science and Engineering, volume 50 , p. 283,1973 .

20. J. E. Garnier and S. Begej, Ex-Reactor Determination of Thermat Gap ano contact conouctance Between Uranium Dioxide: Zircaloy-4 Interfaces, NUFEG/CR-0330, PNL-2696, April 2979.*

21. B. A. Finlayson, The Method of weighted Residuals and variational Principles, New York: Academic Press, 1972.

22. H. H. Mcadams, Heat Iransmission, 34th Edition, New York: MCGraw-Hill Book Company, Inc., 1954. 
23. R. W. Garner et al., Gap Conductance in Test Series-2 Test Results Report for Tests $6 C 2-1,6 C 2-2$, and GC 2-3, NUREG/CR0300 , TREE-1266.**

24. A. Mendelson, Plasticity: Iheory and Apolications, New York: The Macmillan Confany, 1968.

25. U. R. Coleman and E. T. Laats, FRAP-T3, A Computer Code for the Transient Analysis of Oxide Fuel Rods - Model Assessment Report, NUKEG/CR-0535, TREE-1320, Rebruary $1979 . *$

26. F. Hill, A Theory of the Yielding and Plastic flow of Anisotropic Metals, proceedings of the Royal Society of Lonoon, Vol $1434, P P .281-297,1948$.

27. P.j. Pankaskje, Irradiation Effects on the Mechanical Properties of Zirconium on oilute Zirconium alloys: a Revien, $B N-F A-61 B$, JuIy 1976.

?d. m. Nausch ano r. E. Panisko, ANS54: A Computer Subroutine for Predicting fission Gas Release, NUREG/CR-1213, PNL-3077, August $1979 . \star$

29. C. E. Beyer, C. R. Hann, Prediction of Fission Gas Release from UO 2 Fuel, BNWL-1875, November 1974. 
30. J. Weisman et al., Fission Gas Release from UG 2 Fuel Rod with Time Varying Power Histories, ANS Transactions, 12,2 November 1969.

31. J. Rest, GRASS-SST: A Comprehensive Mechanics Model for the preaiction of Fission Gas Behavior in UO 2 Based Fuels curino Steady State and Transient Conditions, NUREG/CR-0202, ANL-7E-53, June $1978 . \star \star$

32. R. Soulhier and M. J. F. Notley, Effect of Power Changes on Fission product Gas Release fram UQ Fuel, Nuclear applications, Volume 5, P. 296.

33. A. H. Booth, A Method of Calculating Fission Gas Diffusion from $U Q_{2}$ Fuel and ItS Application to The $x-2$ Loop Test, AECL-496, CROC $-721,1957$.

34. H. M. Ferrari, Nitrogen Release from U0, Pellets at Elevated Temperatures, Nuclear Science and Engineering, 17, 4, Decemoer 1963.

35. H. H. Ferrari, Diffusion of Nitrogen in Uraniua Dioxide, Journal Df Nuclear Materials, 12, 2, 1964.

36. P. R. Bevington, Lata Reduction and Error Analysis for the Pnysical Sciences, New York: McGraw-Hill Book Company, 1969.

37. P. H. Kreyns, G. L. Spahr, J. E. McCauley, an Analysis of Iodine Stress Corrosion Cracking of Zircaloy-4 Tubing, WAPD-TM-1248, February 1970 . 
38. K. P. Tucker, P. H. Kreyns, J. J. Kearns, The Effects of Internal Surface Flaws, lodine Concentration and Temperature on the Stress Corrosion Cracking Benavior of Zircaloy-4 Tubing, HAPD-IM-1248, Fetruary 1976.

39. R. L. Plackett and J.P. Burman, The Design of Opotimum Multifactorial Experiments, Biometrika, 33, p. 305-325, 1946.

40. L. J. Siefken et al., FRAP-TS: A computer code for the Transient Analysis of Oxide fuel Rods, NUREG-CR-0840, TREE-1281, June 1979.*

41. J.P. C. Kleijnen, Statistical Techniques in Simulation - Part II, Marcel Dekker, Inc., Publisher 1975.

ॠAvallable for purchase from the NRC/GPO Sales Program, U.S. Nuclear Regulatory Commission, Washington, DC 20555, and the National Technical Information Service, Springfield, VA 22161,

* Available for purchase from the National Technical Information Service, Springfield, VA 22161. 
AP PENCIX A

INPUT ANE LUTPUT DESCRIPTION 
APPENLIX A

INPUT AND DUTPUT UESCRIPTIUN

Appenaix a descrites the input necessary to run FRAPCGN-2 and how to interpret the output data. The makeup of the input datadeck consists of:

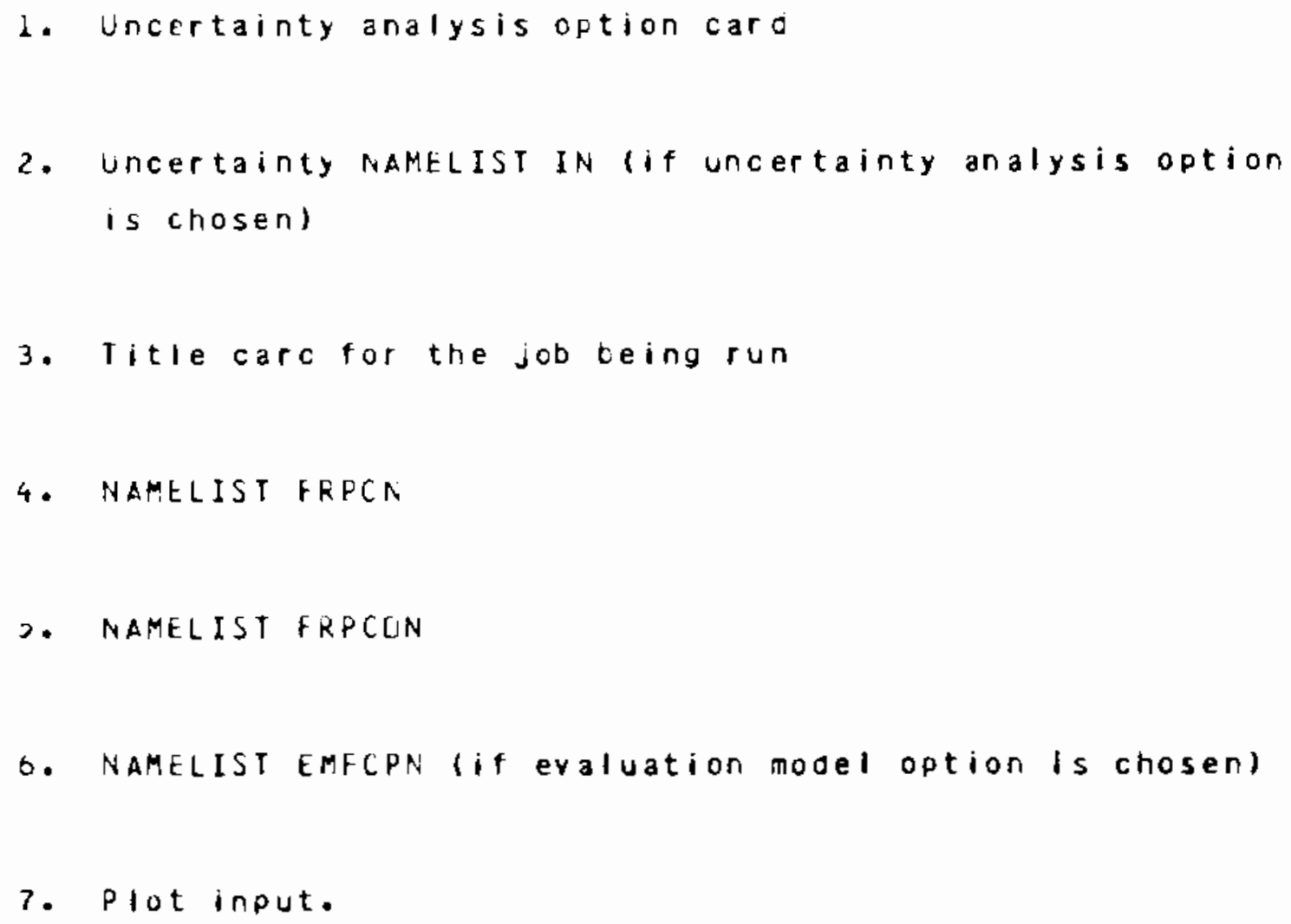

Ine stanaro input includes the uncertainty analysis option card, the titie caro, and tho NAMELISTs, FRPCN and FRPCON. GPtional input - includes the NAMElist in, NAMElist emfCPN, and the plot input.

Section 1. of this appendix describes the standard input, which is in NAMELIST format. Section 2. describes the optional 
input. Section 3. Fresents the output format and Section 4. describes the contral languaye necessary to run the code on the CDC CYBER computer.

\section{STANDARD INPUT}

1.1 USE OF NAMELIST.

The standaro infut to FRAPCQN-2 is in the form of two NAMELISTs, FRPCN and FRPCON. Tre use of NAMELIST input requires that the user foltow a prescritec input format. Within certain restrictions, nowever, great flexitility is provided for designing an input deck.

The input format requires that:

1. The first character on every card be left blank.

2. The first cara of a MAMELIST data set must contain a "s" in column 2, immediately followed by the appropriate NAMELIST name.

3. The last entry of a data set must be a "\$".

4. The data items must be separated ty commas (tre comma should follow imadiately after the constant).

The form of the data items in an input record is

SYERoLig-Uane = Gogstant, 
The symbolic name may be a variable name or an array element name. subscripts must be integer numbers. The egastagt may be an integer or real number.

1.2 DATA DECK FQRMULATIUN.

The caros necessary to create a standaro input deck for FRAPCON-2 include the uncertainty analysis option card, the title card, NAMELIST FKPCN, and NAMELIST FRPCON.

The uncertainty analysis card is used to input the choice of whether or not the uncertainty analysis option is to be run. If the option is to be used, a "l" is placed in column 5 and NAMELIST IN follows. If the option is not to be used, a "o" is placed in column 5. If the uncertainty analysis option is not used, the uncertainty analysis card is followed by the title card(s). There is no limitation on the length of the title or the number of title cards used, but only the first 40 characters of the first card will be printed by the cooe. The titte card is followed first ty NamelIST FRPCN, and trien by NAMELIST FRPCON. Evaluation models are used if the value of the variable IMSHCH is not equal to zero. If plot options are chosen, additional cards are added after NAMELISI FRPCCN.

What follows is a description of the variables comprising NAMELIST FRPCN. These variables are used by the code to describe the physical space in the computer where information will be stored. 
NAMELISI_ZERECE

Vaciacle-vane

LAXSYM

I M

MECHAN

ivA

NC

NF

$N R$
Cescrietion_ang_testciction

Controls AXISYM Option; use only with MECHAN $=3$

$$
\begin{aligned}
& =1 \text {, AXISYM is used } \\
& =0 \text {, AXISYM is not used }
\end{aligned}
$$

Number of power-time steps igreater than 1)

Cortrols choice of mechanical model

0

×1, PELET-RAUIAL

$\approx 2$, FRACAS- 1

$=3$, FRACAS-II

Number of axial regions (may vary f(cmi 3 to 18$)$

Number of radial nodes in cladding; used only if MECHAN=1 (greater than 1)

Number of radial nodes in fuel; used only if MECHAN=1 (areater than 1)

Number of $r$ adial nodes in fuel;

used for thermal calculations

(recommended value is 11 ) 
NGASR

$$
\begin{aligned}
& \text { Cortrols choice of yas release mooel } \\
& =-2 \text {, FASTIGRASS Or GRASS } \\
& =-1 \text {, Bootr oiffusion } \\
& \text { = } 0 \text {, Maclonalo-heisman } \\
& \text { = 1, beyer-Hann with NRC high turnup } \\
& \text { correction factor } \\
& =2 \text {, Beyer-Hann without NRC high turnup } \\
& \text { correction tactor } \\
& \geq 6 \text {, ANS-5.4; NGASR is used as } \\
& \text { the number of radial regions } \\
& \text { in the fuel for the gas release } \\
& \text { calculations }
\end{aligned}
$$

When cnoosiny values for the variables in NAMELIST FRPCN the user inust consider that more detailed modeling will result in increased computer core storage requirements and increased running time. Those variaules which directly influence the running lime and core storage requirements include IY, NA, AND NR (NF and NC if MECHANAl). The influence of the MLCHAN and NGASR option choices is best determined by trial for the specific problem being modeled.

The caro immediately following the SEND card of NAMELIST FRPCN will be the first caro of NAMELIST FRPCON. NAMELIST FRPCON contains the actual rod parameters, poner history, ete., and consists of the variables listed belcw. The user has the option to Input English or SI units. Each input variable lists the appropriate English or SI unit in the variatle aescription. Thesemust be input with those units. 


\section{NAMELISI ERPCON}

Variagle Name

CLMP

$C P L$

$0 \mathrm{CI}$

DCO

DE

DELTAZ

$\triangle E N$

DISHSO
Cescriotion and Restrictions

Default value

Plutonium oxide content of fuel

0

(neight percent)

Plenum length $(i n,, m\}$

Inside oiameter of cladding (in.,m);

input a value for each axial

region if IVARDH $=2$

Lutside diameter of cladding (in.,m);

input a value for each axial

region if IVARDM $=2$

Equivalent heated diameter $($ in.,m $)$;

input a value for each axial

region if IVARDM $=2$

Array containing lengths of axial

secments (in.,m); variable only if

IVARLIM $=1$

Fuel density as percent of theoretical; $10.97 \mathrm{gm} / \mathrm{cc}$ is assumed theoretical denstty

Dish shoulder width; pellet radius

minus dish radius $(i n ., m)$ 
DP

DSPG

DSPGW

ENRCH

FA

FGPAV

FLUX

GO

HDISH

HPLT
Liameter of pellet (in.,m); input a value for each axial region if IVARDM $=2$

Cutside diameter of spring (in.,m)

Diameter of spring wire $(i n ., m)$

Fuel enrichment (weight percent)

Axial power profile peak-to-average ratio

$1 \cdot 0$

$=1.0$, if GMPY = average

$>1.0$, if GMPY = peak

Initial fill gas pressure ipsia, $N / m^{2}$ )

Fast neutron flux (neutrons $/ m^{2} . s$ )

$6 \times 10^{17}$

Mass flon rate (lo/hr. ft $\left.{ }^{2} \mathrm{~kg} / \mathrm{s} \cdot \mathrm{m}^{2}\right)$

0 see NSP; if go:0, cladoing surface temperature $=T w$ at all axial nodes

Depth of pellet end dish (in.,m)

Height of pelfet $(i n ., m)$ 
ICH

$$
\begin{aligned}
& \text { Index for cladoing material } \\
& =2, \text { zircaloy-2 } \\
& =4, \text { zircaloy-4 }
\end{aligned}
$$

IDXGAS

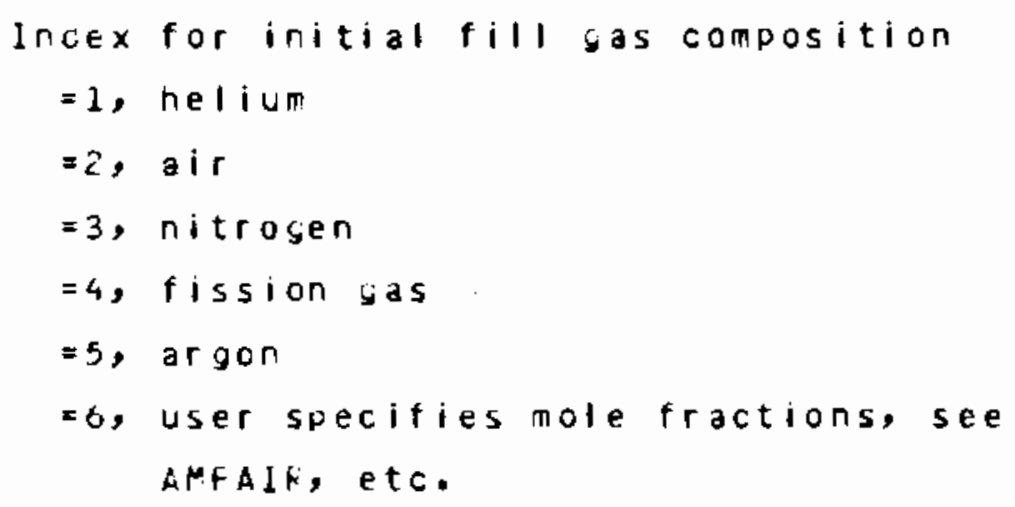

$\operatorname{MSNCH}$

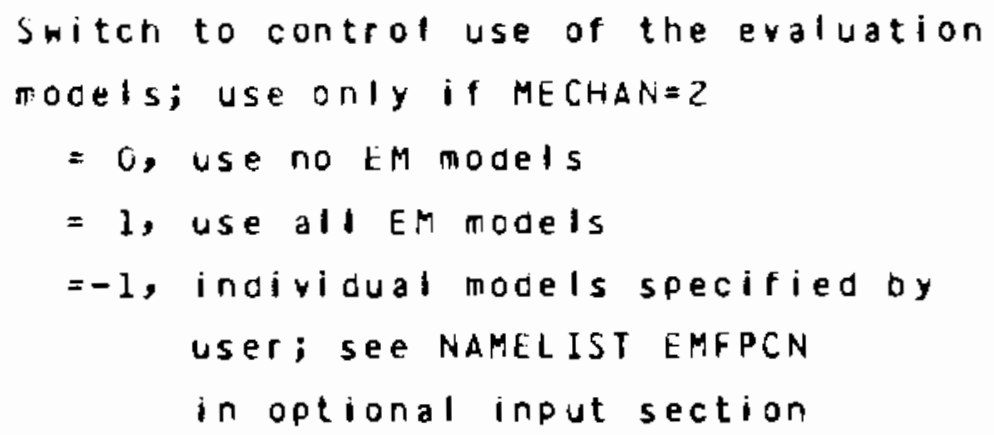


Variagle vame

10

I VARUM

$J[L P R$

$J N$

US T

Index for axial power shape o

$=0$, user input (see FA, QF)

- I, cosine shape calculateo by code crpy must be average poners; see $F A$

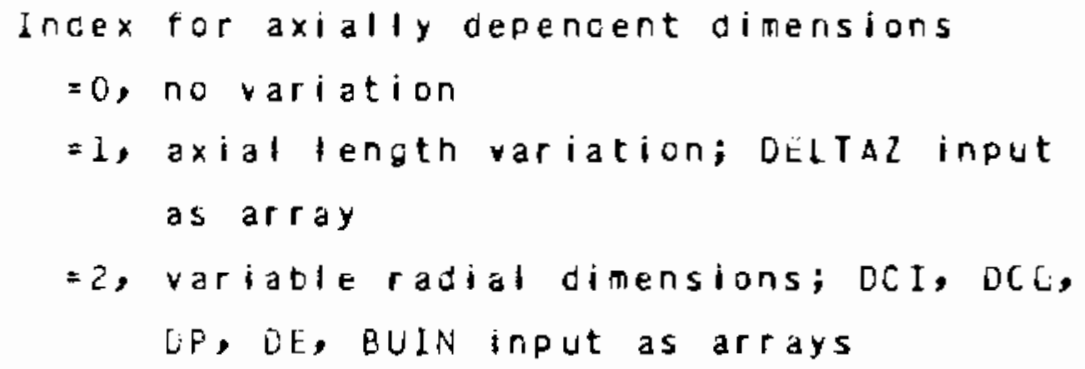


Vociable Name

NiPT

NSP

ivUNITS

$p 2$

JF

OMPY

$R C$
Cutput option

c

$=0$, tull output

$=3$, summary page output

Sitch for time dependent parameters

0

$=0$, constant parameters

$=1, P 2, T h$, ano GD must be input as

arrays versus time array

Specifies infut unit type

0

=0, metric units

$=1$, English units

System pressure (see NSP)

(psia, $N / m^{2}$ )

Axial power profile factors; up to 8

shapes containing JN entries for each

shape; May te normalized to peak or average

Foner; see $H A$, IQ, OMPY

Linear heat rating array versus time

array $(k w / f t, k w / m)$.

CMfy values may be either peak or average

poners. If MECHAN $=1$, OMPY should not

change more than $5.0 \mathrm{~kW} / \mathrm{m}$ (1.5 $\mathrm{kw} / \mathrm{ft})$

per time step.

Racius of pellet annulus $(i n, m)$

0.0 


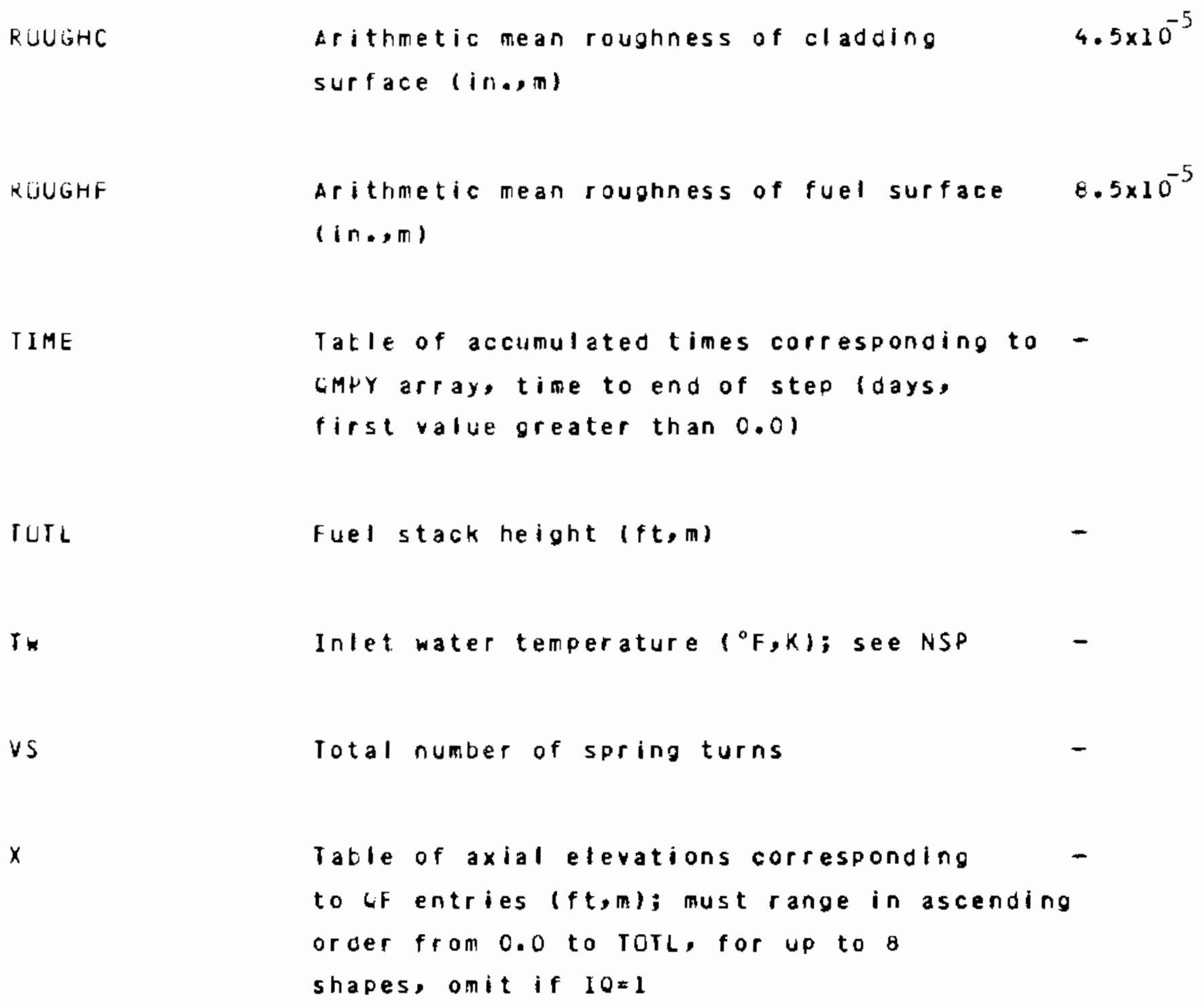

The following variables, also contailed in NAMELIST FRPCON, are basically input options rather than requirements. The user may wish to use the default values for mast of these variables. 
Default Name

AMFAIR

AMFARG

$A M F F G$

AMFHE

AMFH2

AMFH 20

AMFKRY

AMFN2

AMFXE
Absolute mole fraction of air;

0.0

WSed Only if IOXGAS =

Dbsolute mole fraction of arjon;

0.0

used onty if IDXGAS = 6

Absolute mole fraction of fission gas;

$0 . \mathrm{C}$

used onfy if ILXGAS = 6; use only if

$\triangle M F K R Y=0.0$ and $A M F K E=0.0$

Absolute mole fraction of hetium;

$0 . \mathrm{C}$

useo only if IDXGAS =

Absolute mole fraction of nyarogen;

0.0

usea only if IDXGAS $=0$

Absolute mole fraction of steam;

0.0

useo onty if ICXGAS = 6

ADsclute mole fraction of krypton;

0.0

USec only if ICXGAS = 6

Absolute mole fraction of nitrogen;

0.0

used only if IOXGAS = 6

Absolute mole fraction of xenon;

0.0 
$B \in T A$

Buis

CATEXT

CLOAK

$C P$

CR

CKUT

CROTK

CREPHK

$\operatorname{CTMAX}$ variable not currently used.

Initial fuel burnup (Mwd/MiU, Mws/kg);

also consider inputting AMFFG; may be

axially defendent array if IVARDM $=2$

Texture factor: fraction of cladaing cells hith basal poles parallel to the tude axis

Cold work of the cladding, fraction

0.2

Anisotropy coefficient; used with MECHAN=1 1.0

Anisotropy coefficient; used with MECHAN=1 1.

Initial cruathickness $(\mathrm{mil}, \mathrm{m})$

1.0

Lrud builoing rate (milhr,m/s)

$1.4 \times 10^{-4}$

onty if 1 COR $=2$

Creep step size for FRACAS-I and FRACAS-II 10.0 (hrs)

Maximum clading temperature attaineo by 386.33

the fuel rod, axial array $(f, k)$ etc. 


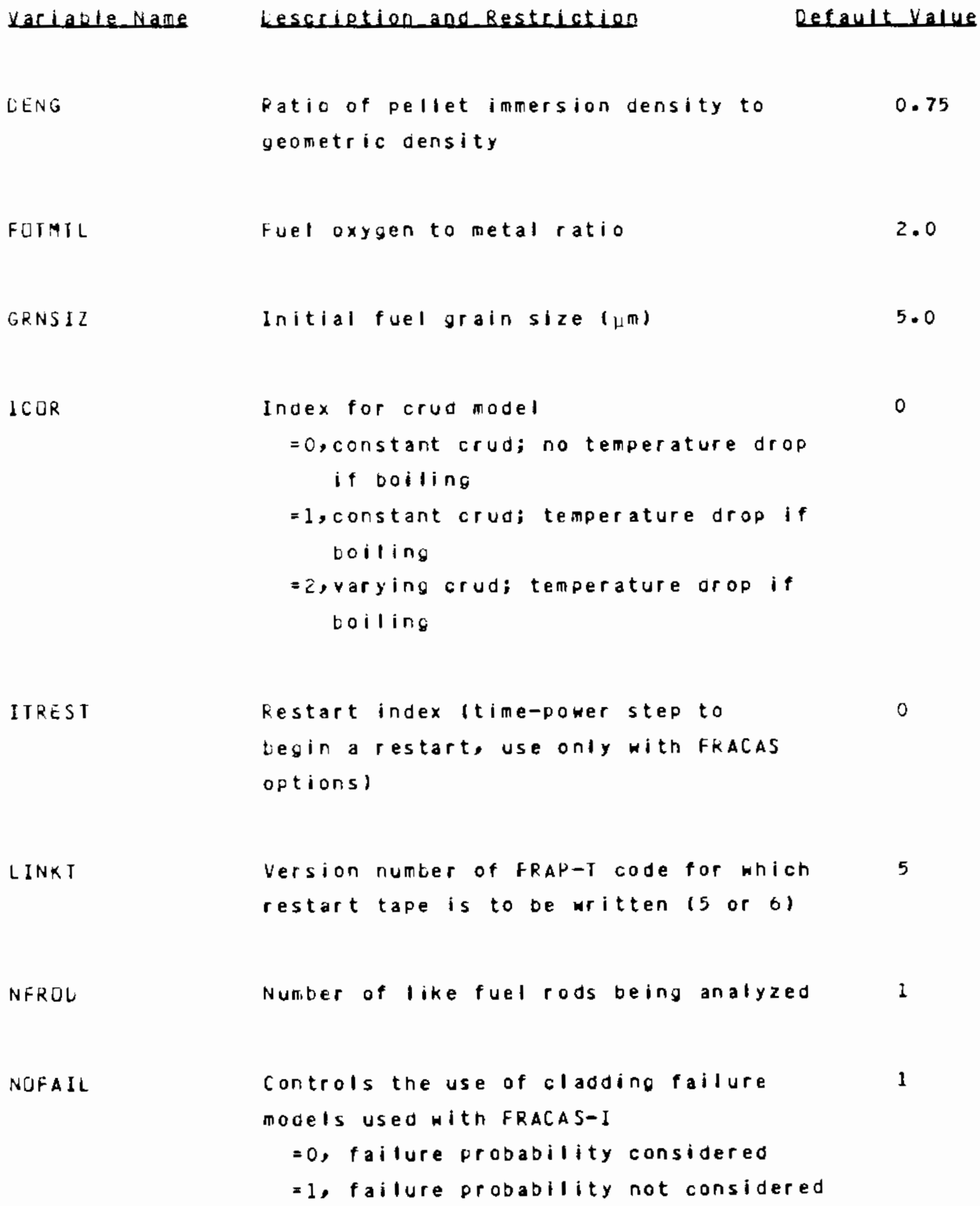


NPCYCL

NPLTAB

NPRINT

NREAD

NRESTR

NTAPE

NUCFC

Number of previous power cycles

0

Parameter on plot abcissa

0

$=0$, time

$=1$, rod average porer

*2, local burnup

$$
\begin{aligned}
& \text { GRASS print index } \\
& =1 \text {, no GRASS output } \\
& =4 \text {, full GRASS output }
\end{aligned}
$$

$$
\begin{aligned}
& \text { FRAPCON restart read } \\
& \quad=0 \text {, no restart read } \\
& =1 \text {, read restart tape }
\end{aligned}
$$

0

$=0$, no restart write

$=1$, write restart tape
FRAP-T restart
$=0$, no restart data stored
$=1$, ata stored each time step for FRAP-T USE

0

\footnotetext{
User specifled collapse failure crlterion

$=0$, collapse mode is not considered

$=1$, collapse mode is considered
}

0 
Fuel initial nitrogen content (ppm) 15.0 of fuel stack to plenum, one value e tc. for each axial power profile; see $Q F$

RAPQW

Facial power profile, applied at 1, I, Louncaries of equal volume rings in order etc. from surface to center; II values are required, normalized to an average value of 1.0 , used only if IPLANT $\neq-$ I

RSNTK

Absolute change in fuel density due to 0.0 thermal resintering $\left(\mathrm{kg}_{\mathrm{g}} \mathrm{m}^{3}\right.$ ) 
The optional infut is comprised of three groups, the uncertainty analysis input, the evaluation model input, and the plot input.

2.I UNCERTAINIY UPTIGN.

As noteo previously, the uncertainty option is used when a "l" is placeo in column $b$ of the uncertainty analysis card. when the option is selecteo for use, tre uncertainty analysis option card is followed by the NAMELIST IN. The varlaties contajned in IN are described Delow.

\section{NAMELISI IN}

Variable vame

FACT DR(K)
Cescription and Restrictions

Flag for adaitive or multiplicative uncertainty factors.

$=c$, additive

$=1$, multiplicative (default)
Lefault value 
$C(I, J, K)$

Uncertainty factors. Factors are 0.0

aescribed by polynonials of up to third etc.

order in temperature (I) in four temperature

ranges (J) for approximately 50 factors (K).

Values are ore standard deviation.

Multiplicative factors snould de inout as a

decimal fraction. For example, an

uncertainty factor of 1.25 indicating a one

sigma uncertainty of 25 shovis be input

as 0.25 . The code will ads the 1.00

when usea

$T L(J, K)$

Upper temperature linits describing

ranges applicable to sncertainty

factor polynomials. If no temperature

range uppermost limit, enter 3 .

$\operatorname{LRES}(V)$

List of responses fron rable $A-l$.

Order is not important. Individual

responses may be listed more than once.

For example, a given response may oe desired

at nore than one axial node. Not to exceed

100 total responses.

$\operatorname{NODE}(N)$

List of axial nodes corresponding

LRES(N). List must naten LRES(V)

one for one. 
TabIE A-1. DEFAJLT UNCERTAINTY FACTURS

\begin{tabular}{|c|c|c|c|c|}
\hline LFAO & Sour $\approx e$ & $\begin{array}{l}\text { Additive (A) or } \\
\text { Multiplicative }(M) *\end{array}$ & ractor & $\begin{array}{l}\text { Temp. } \\
\text { Range }(k) \\
\end{array}$ \\
\hline$i \cdot$ & Fued specific heat & M & $\begin{array}{l}1.02 \\
1.012+1.0 E \\
-5 \times 1 \\
2.06\end{array}$ & $\begin{array}{l}T<500 . \\
500 .<T<3000 . \\
3000 .<T\end{array}$ \\
\hline $2 \cdot$ & $\begin{array}{l}\text { Fuel thermal } \\
\text { conductivity }\end{array}$ & $A$ & 0.2 & - \\
\hline 3. & Fuel erissivity & M & 1.10 & - \\
\hline 4. & $\begin{array}{l}\text { Fuel thermal } \\
\text { expansion }\end{array}$ & $A$ & $\begin{array}{l}0.253-6 \times 1 \\
0.00125\end{array}$ & $\begin{array}{l}T<500 \\
500 .<i\end{array}$ \\
\hline 5. & $\begin{array}{l}\text { Fuel elastic } \\
\text { modulus }\end{array}$ & $A$ & $\begin{array}{l}0.3 E 11 \\
(\dot{P})\end{array}$ & - \\
\hline 6. & Poisson's ratio & 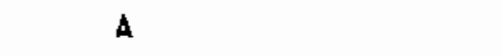 & 0.074 & - \\
\hline 7. & Fuel creep & - & $(T B D) *$ & - \\
\hline$\theta$. & $\begin{array}{l}\text { Fuel fracture } \\
\text { strength }\end{array}$ & A & $(\dot{p} \mathrm{a})$ & - \\
\hline 9. & Fuel swelling & M & 1.50 & - \\
\hline 10 & Fuel restructuring & - & (T⿺辶寸) & - \\
\hline 11. & Fuei densification & - & $(T B)$ & - \\
\hline 12. & $\begin{array}{l}\text { Fission gas } \\
\text { release }\end{array}$ & - & $(T B D)$ & - \\
\hline 13. & $\begin{array}{l}\text { Cladding specific } \\
\text { heat }\end{array}$ & $A$ & $\begin{array}{l}10 \cdot(\mathrm{J} / \mathrm{kg} \cdot \mathrm{K}) \\
10: \\
25: \\
100 .\end{array}$ & $\begin{array}{l}T<300 . \\
300 .<T<1390 . \\
1090 .<T<1300 . \\
1300 .<T\end{array}$ \\
\hline 14. & $\begin{array}{l}\text { Cladding thermal } \\
\text { conductivity }\end{array}$ & $\Delta$ & $(* 01, k)$ & - \\
\hline 15. & $\begin{array}{l}\text { Zircoxide } \\
\text { emissivity }\end{array}$ & 4 & 0.10 & - \\
\hline
\end{tabular}


Table a-1. (Continued)

\begin{tabular}{|c|c|c|c|c|}
\hline$\angle F A C$ & Source & $\begin{array}{l}\text { Alditive (A) or } \\
\text { mutiplicative (M)* }\end{array}$ & Factcr & $\begin{array}{l}\text { temp. } \\
\text { kange }(<)\end{array}$ \\
\hline ló. & $\begin{array}{l}\text { Zirc oxide tnermal } \\
\text { conductivity }\end{array}$ & $A$ & 1.20 & - \\
\hline 17. & $\begin{array}{l}\text { Cladoingaxial } \\
\text { therinal expansion }\end{array}$ & $M$ & $\frac{1}{1}: 20$ & $\begin{array}{l}T<1073 \\
1073 .<T\end{array}$ \\
\hline 18. & $\begin{array}{l}\text { Cladainjodanetral } \\
\text { thernal expansion }\end{array}$ & $i$ & $\frac{1}{1} \cdot 20$ & $\begin{array}{l}1<1073 \\
1073<1\end{array}$ \\
\hline 19. & $\begin{array}{l}\text { Cladion elastic } \\
\text { nodulus }\end{array}$ & $y$ & $\frac{1}{1} \cdot \frac{1}{20}$ & $\begin{array}{l}T<1003 \\
1083 .<1\end{array}$ \\
\hline 20. & $\begin{array}{l}\text { Cladoing strength } \\
\text { coefficient }\end{array}$ & 4 & $\begin{array}{l}27: 50 \\
(P a)\end{array}$ & - \\
\hline 21 & $\begin{array}{l}\text { cladding } \\
\text { circumferential }\end{array}$ & A & $\begin{array}{l}0.03 \\
-0.91+1.25 \\
5-3 \times 1 \\
0.11\end{array}$ & $\begin{array}{l}T<800 . \\
900 .<T<1170 \\
1170 .<T\end{array}$ \\
\hline 22 & $\begin{array}{l}\text { Cladding Meyer } \\
\text { nardness }\end{array}$ & - & $(130)$ & - \\
\hline 23. & $\begin{array}{l}\text { Gladding creep } \\
\text { rate }\end{array}$ & - & $(\pi B J)$ & - \\
\hline 24. & $\begin{array}{l}\text { Lladjing poisson's } \\
\text { ratio }\end{array}$ & $A$ & 0.025 & - \\
\hline 25. & Cladding oxidation & M & $\begin{array}{l}1.175 \\
1.065\end{array}$ & $\begin{array}{l}1<1523 \\
1523<1:\end{array}$ \\
\hline 26. & $\begin{array}{l}\text { Gas thernat } \\
\text { conductivity }\end{array}$ & M & 1.25 & - \\
\hline 27 & Gas viscosity & $M$ & 1.25 & - \\
\hline 23 & bap neat transfer & $M$ & $1 \cdot 2 j$ & - \\
\hline 29. & $\begin{array}{l}\text { A pha-Beta tran- } \\
\text { sition tenperature }\end{array}$ & 4 & $10 .(k)$ & - \\
\hline
\end{tabular}


Table $4-1$. (Continued)

\begin{tabular}{|c|c|c|c|c|c|}
\hline$\angle F A S$ & Sourse & Mudttive (A) or & Factor & $\begin{array}{l}\text { Temk. } \\
\text { kange }\end{array}$ & $1<1$ \\
\hline 30. & $\begin{array}{l}\text { pellet stack } \\
\text { nelght }\end{array}$ & $r_{1}$ & 1.001 & - & \\
\hline 31. & $\begin{array}{l}\text { ¿ladding outer } \\
\text { jianeter }\end{array}$ & $M$ & 1.001 & - & \\
\hline 32. & Fuel density & M & 1.0007 & - & \\
\hline 33. & $\begin{array}{l}\text { Pellet snoulder } \\
\text { radius }\end{array}$ & 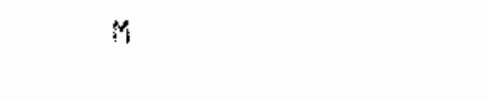 & 1.034 & - & \\
\hline 34. & $\begin{array}{l}\text { Metlet dish } \\
\text { depth }\end{array}$ & M & 1.034 & - & \\
\hline 35. & Pel tet height & M & 1.001 & - & \\
\hline 30. & $\begin{array}{l}\text { Pellet dish } \\
\text { volume }\end{array}$ & is & 1.034 & - & \\
\hline 37. & $\begin{array}{l}\text { Peliet outer } \\
\text { radius }\end{array}$ & $M$ & 1.001 & - & \\
\hline 38. & $\begin{array}{l}\text { Cladoiny inner } \\
\text { radijs }\end{array}$ & $M$ & 1.001 & - & \\
\hline 39. & $\begin{array}{l}\text { Cladoing outer } \\
\text { radius }\end{array}$ & 19 & 1.001 & - & \\
\hline 40 & Cladding roughness & $i 1$ & 1.100 & - & \\
\hline 41 . & Fuel roujnness & M & 1.100 & - & \\
\hline 42 & $\begin{array}{l}\text { Amount of gas } \\
\text { in rod }\end{array}$ & $M$ & 1.034 & - & \\
\hline 43. & plenum volume & $\mathrm{Mi}$ & 1.001 & - & \\
\hline 44. & cold pressure & M & 1.034 & - & \\
\hline
\end{tabular}


Iaole a-1. (Continued)

\begin{tabular}{|c|c|c|c|c|}
\hline LFAC & Sour 2 e & $\begin{array}{l}\text { Additive }(A) \text { or } \\
\text { Multiplicative }(M) \neq\end{array}$ & Factor & $\begin{array}{l}\text { Temp. } \\
\text { Range }\end{array}$ \\
\hline 45. & $\begin{array}{l}\text { Mole fractions of } \\
\text { of gas components }\end{array}$ & - & $(T E J)$ & - \\
\hline 46. & Unused & - & - & - \\
\hline 47. & $\begin{array}{l}\text { Initial temp- } \\
\text { erature estinate }\end{array}$ & - & $(T B D)$ & - \\
\hline 48 & Pomer history & $M$ & $1.0 j 0$ & - \\
\hline 49. & $\begin{array}{l}\text { ANS decay } \\
\text { heat curve }\end{array}$ & M & 1.067 & - \\
\hline 50. & Unused & - & - & - \\
\hline 51. & EHF factor & $M$ & 1.080 & - \\
\hline 52. & Jnuseo & - & - & - \\
\hline b3. & Unused & - & - & - \\
\hline 54. & Unused & - & - & - \\
\hline 55. & Jnused & - & - & - \\
\hline 50. & Jnused & - & - & - \\
\hline 57. & Unuseo & - & - & - \\
\hline 58. & Unused & - & - & - \\
\hline 59. & Unused & - & - & - \\
\hline 00 & Jnused & - & - & - \\
\hline 61. & Plenum length & - & $(T(1))$ & - \\
\hline 62. & $\begin{array}{l}\text { Equivalent heateo } \\
\text { diameter }\end{array}$ & - & $(T B D)$ & $\overrightarrow{-}$ \\
\hline
\end{tabular}


Taole $A-1$. (Continued)

\begin{tabular}{|c|c|c|c|c|c|}
\hline LFAC & Sourse & $\begin{array}{l}\text { Adoitire (A) or } \\
\text { Multiplicative }(M) *\end{array}$ & Factor & $\begin{array}{l}\text { Temp. } \\
\text { Range }\end{array}$ & $(<)$ \\
\hline 63. & $\begin{array}{l}\text { Diameter of } \\
\text { spring, autside }\end{array}$ & - & $(T B O)$ & & \\
\hline 64. & $\begin{array}{l}\text { Diameter of } \\
\text { spring wire }\end{array}$ & - & $(T B O)$ & - & \\
\hline 65. & Fuel enrichment & - & $(T O D)$ & - & \\
\hline 60. & Mass flow rate & - & $(\mathrm{T} \Delta U)$ & - & \\
\hline 67. & system pressure & - & $(180)$ & - & \\
\hline 68. & $\begin{array}{l}\text { Axial heat } \\
\text { Normalization } \\
\text { Factors }\end{array}$ & - & $(T B)$ & - & \\
\hline 69. & $\begin{array}{l}\text { Fuel stack } \\
\text { neisht }\end{array}$ & - & $(\mathrm{T} \theta)$ & - & \\
\hline 70 & $\begin{array}{l}\text { Injet water } \\
\text { temperature }\end{array}$ & - & $(T \unlhd D)$ & - & \\
\hline 71. & $\begin{array}{l}\text { Total spring } \\
\text { turns }\end{array}$ & - & $(T E D)$ & - & \\
\hline 72. & $\begin{array}{l}\text { Initiai fuel } \\
\text { burnup }\end{array}$ & - & $(T B))$ & - & \\
\hline 73. & Texture factor & - & $(\mathrm{TBD})$ & - & \\
\hline 74. & Cold work & - & $(T 03)$ & - & \\
\hline 75. & $\begin{array}{l}\text { Weight percent } \\
\text { puo }\end{array}$ & - & $(T B D)$ & - & \\
\hline 76. & $\begin{array}{l}\text { Initial crua } \\
\text { thickness }\end{array}$ & - & $(T B O)$ & - & \\
\hline 77. & $\begin{array}{l}\text { Crud buildup } \\
\text { rate }\end{array}$ & - & $(T B D)$ & - & \\
\hline 78. & Porosity correction & - & $(T B O)$ & - & \\
\hline
\end{tabular}


Taole $a-1$. (Cantinuea)

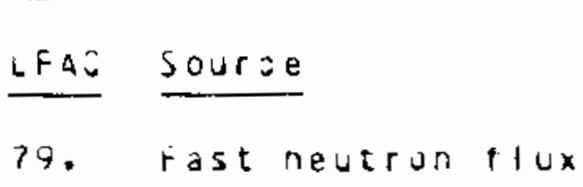

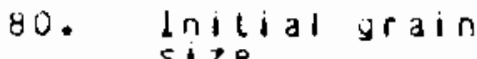

81. Fuel initial

water content

Q2. Fuel initial

nitrogen content

d3. vornalized neat flux at top of fuel stack

84. Pellet core $r a d i$ is

85. Fissian yas atoms per loj fissions

4o. Fuel sintering temperature

d7. iladaing oxiae

failure criterion

80. Unused

89. Jnused

90. Jnused

91. jnused

92. Jnuseo

73. Jnusea

74. Unused

75. Unused

Adutire (a) or

multinjicativ? (y)

Factor

Tent?

Rarye $(<)$

$(100)$

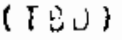

( $(\mathrm{T})$

(Td S)

$-$

$-$

(T)

(15)

( 13.$)$

(T日)

$-$

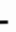


Tajo A-1. (Continueo)

\begin{tabular}{|c|c|c|c|c|}
\hline$\angle F A S$ & jourse & $\begin{array}{l}\text { Additive (A) or } \\
\text { Multiplicative }(4) * 4\end{array}$ & Factor & $\begin{array}{l}\text { Temp. } \\
\text { kangic } 1<1\end{array}$ \\
\hline 40. & Unusec & - & - & - \\
\hline 97. & Unused & - & - & - \\
\hline 99. & Unuseo & - & - & - \\
\hline 100. & Jnused & - & - & - \\
\hline
\end{tabular}

* Adative (A) or Multiplicative (M) reters to tha nanner in thict the tastor Nas apolied. Ihat is, percentage uncertainties were injltiplicative witeres adolute ucertainties adative.

** (Toj) - to be aetermined. 
LFAC ( $)$

List of factors fron raole $\mathrm{A}-2$

0

to oe included in the analysis. Jroer

is important. Example, (LFA:)=

$2,4,8, j, 5$, the analysis will include

these five factors in tais oroer in the

experimental design and confounding arrays.

LTYPE

Type of anatysis desires.

1

$=1$, linear

$=2$, linear plus foljover

$=3$, linear plus quajratic

$=4$, linear alus foldoves plus quadratic

LPB

Placket-Burman desian tlag

0

$=0$, fractional factorial desion

$=1$, Placket-jurman desizn

$A Y J(L, \prec)$

First four dimensionless central

moments of the uncertaincy factor

distributions asiumed to apply over all

temperature ranges. vornal distribution

is defaut.

IPRINT

Flag for additional experinental

design including design zenerators and

one and two factor aliases.

$=0$, no

$=1$, yes 
Taole A-2. Responses

\begin{tabular}{|c|c|}
\hline LRES & Response \\
\hline 1 & Zircaloy oxide thickness (M) \\
\hline 2 & Net permanent fuel deformation $|m|$ \\
\hline 3 & Cladding plastic noop strain \\
\hline 4 & Cladding plastic axial strain \\
\hline 5 & Clading plastic raoial strain \\
\hline 6 & $\begin{array}{l}\text { Cladcing peak temperature during } \\
\text { operation (k) }\end{array}$ \\
\hline 7 & Jpen porosity \\
\hline 8 & Fuel burnup (Mad/MTU) \\
\hline 9 & Fuel centerline temmeratare $(x)$ \\
\hline 10 & Fuel surface temperature $(1)$ \\
\hline 11 & Cladoing inner surface temperature $(K)$ \\
\hline 12 & Ciadoing outer surtace temperature $(k)$ \\
\hline 13 & Internal gas pressure (Pa) \\
\hline 14 & Raoial gas jap $(m)$ \\
\hline 25 & Gram-moles gas in the rod \\
\hline 16 & Mole fraction of rod gases \\
\hline 17 & Cladding resiodal hoop strains \\
\hline 18 & Cladoing residual axial strains \\
\hline
\end{tabular}


Table a-2. (continues)

\begin{tabular}{ll}
\hline LRES & kesponse \\
20 & Eladaing resigual radial strains \\
21 & Fuel resigual noof strains \\
22 & Fuel residual radial strains \\
24 & Interface pressure (Pa) \\
\hline
\end{tabular}


IFLAG

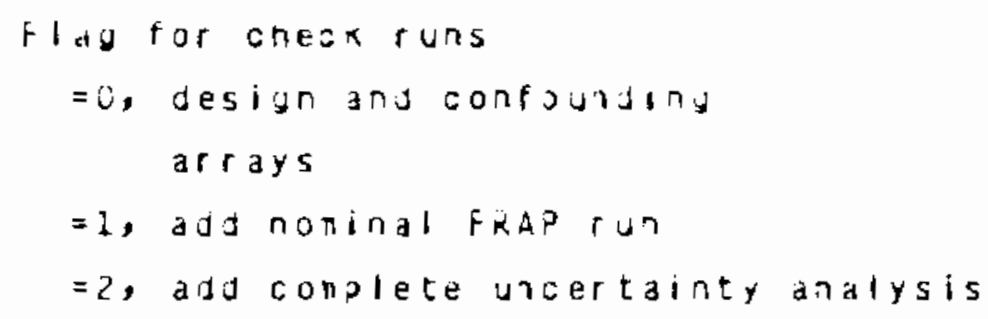

ISTARI

$$
\begin{aligned}
& \text { Hag for restarting from previous } \\
& \text { analysis } \\
& =0 \text {, no } \\
& =1 \text {, yes }
\end{aligned}
$$

2.2 CVALJATIJY MJUEL OPTIJN

4 set of ten th flags are used to specify the EM options. One, flad IMSE:H in the FRPCJN NAFELIST is dsed to set all ootions on or off, or to specify that some combination of the ey mojel options and Dest-estinate 3 nodel options is to de uses.

The inout variatles for tre EMrPSV NAMELIST are given velow. Tney correspond to the nine tm requirenents. All variables are defauted to zero.

The EM input cards follow a BemfPCN caro and end with a SENO card. If i.15d $0 t=0$ or 2 , these cards nust be omitted. 


\section{NAMELISI EMEPCN}

\section{Vaciaole}

Lescription and cestriction

Default value

IMPOWR

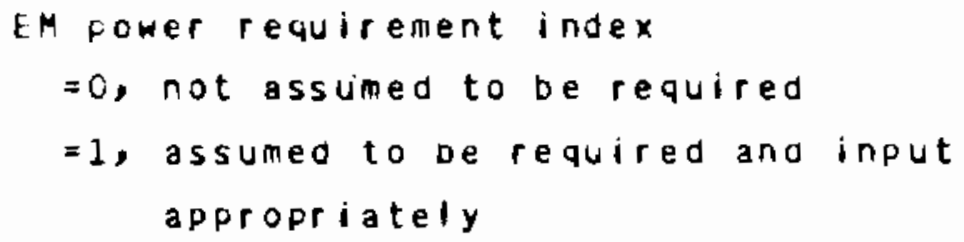

IMFUEL

EM fuel dimensional change index

0

$=0$, BE dimensional changes

- 1 , EM dimensional changes

IMFONS

$$
\begin{aligned}
& \text { EM fwel densification index } \\
& =0, \text { BE densification used } \\
& =1 \text {, EM densification used }
\end{aligned}
$$

IMRELO

$$
\begin{aligned}
& \text { EM fuel relocation index } \\
& =0 \text {, BE relocation used } \\
& =1 \text {, EM relocation used }
\end{aligned}
$$

I MCLAD

$$
\begin{aligned}
& \text { EM cladding deformation index } \\
& =0 \text {, All deformation mechanisms included } \\
& =1 \text {. No permanent deformation included }
\end{aligned}
$$

IMGAPC

$$
\text { EM gap conductance index }
$$

$=0$, EE gap conductance used

I. EM gap conductance used 
Varianle Name

$1 M 93 w 0$

IMENRG

IMFGAS
Description ano Restriction

EM fuet theral conductivity inoex

- 0 , Thermal conouctivity based on $97 \mathrm{~m} / \mathrm{cm}$

$=1$, Thermal conductivity based on $93 \mathrm{H} / \mathrm{cm}$

and uncracked fuel
Refault value

0

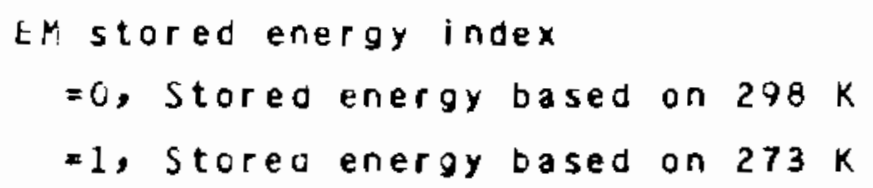

EM stored energy index

$=0$, Stored energy based on $298 \mathrm{~K}$

* 1, Storeo energy based on $273 \mathrm{~K}$

0

2.3 PLOT INPUT

The following is the input data required for plotting. If no plots are desired. follow the "SEND" card of the preceoing input with card l below. If plots are desired, a full set of plot data is required and the folloning plots versus time, power, or burnup isee NPLTAB) will be created for each axial node specified.

1. Cladoing Surface Temperature

2. Fuel centerine Temperature

3. Gas pressure

4. Cladoing Hoop Strain

5. Fuel Axial Elongation

6. Cladding Axial Elongation

7. Local Rod Pomet 


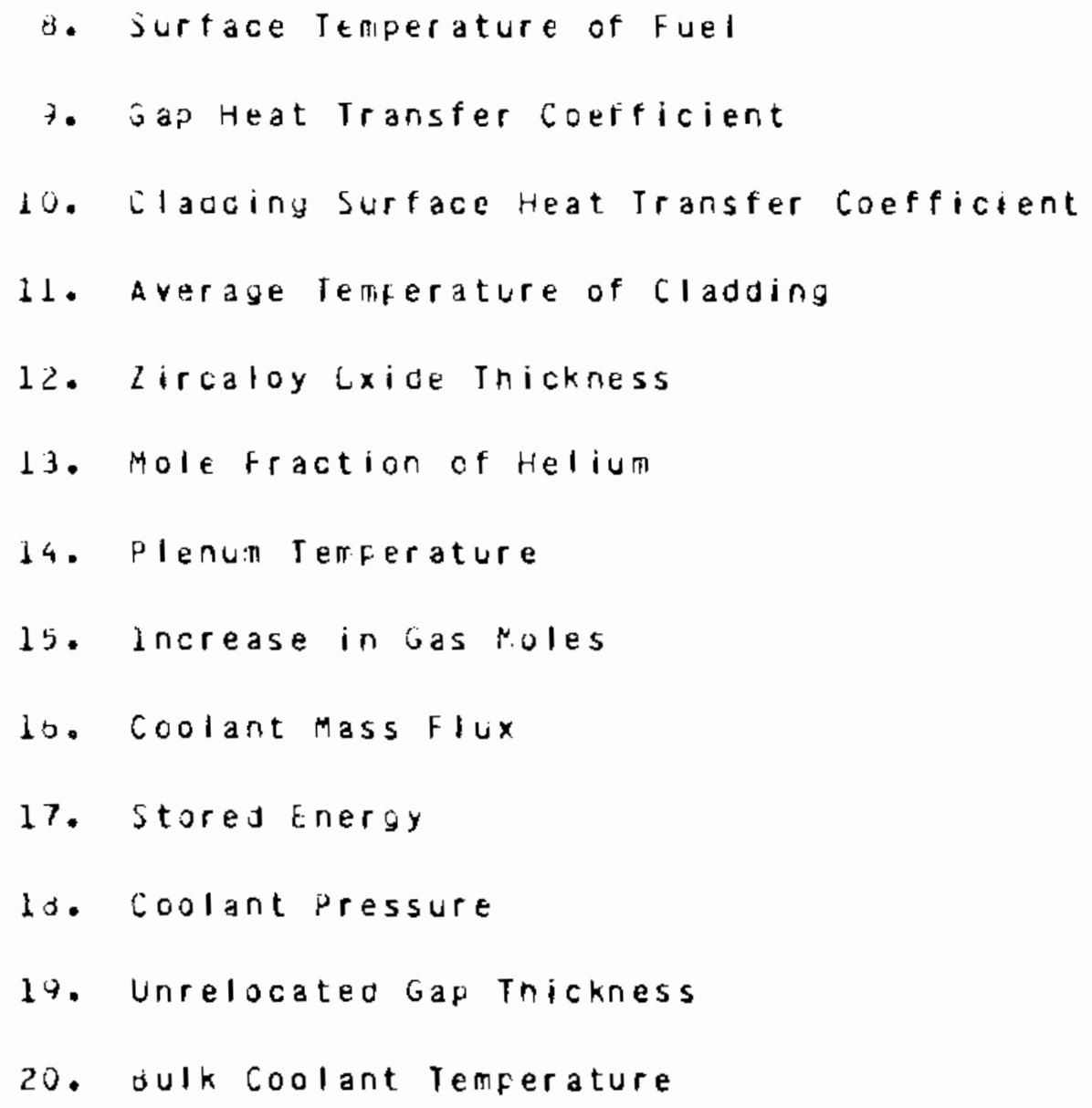




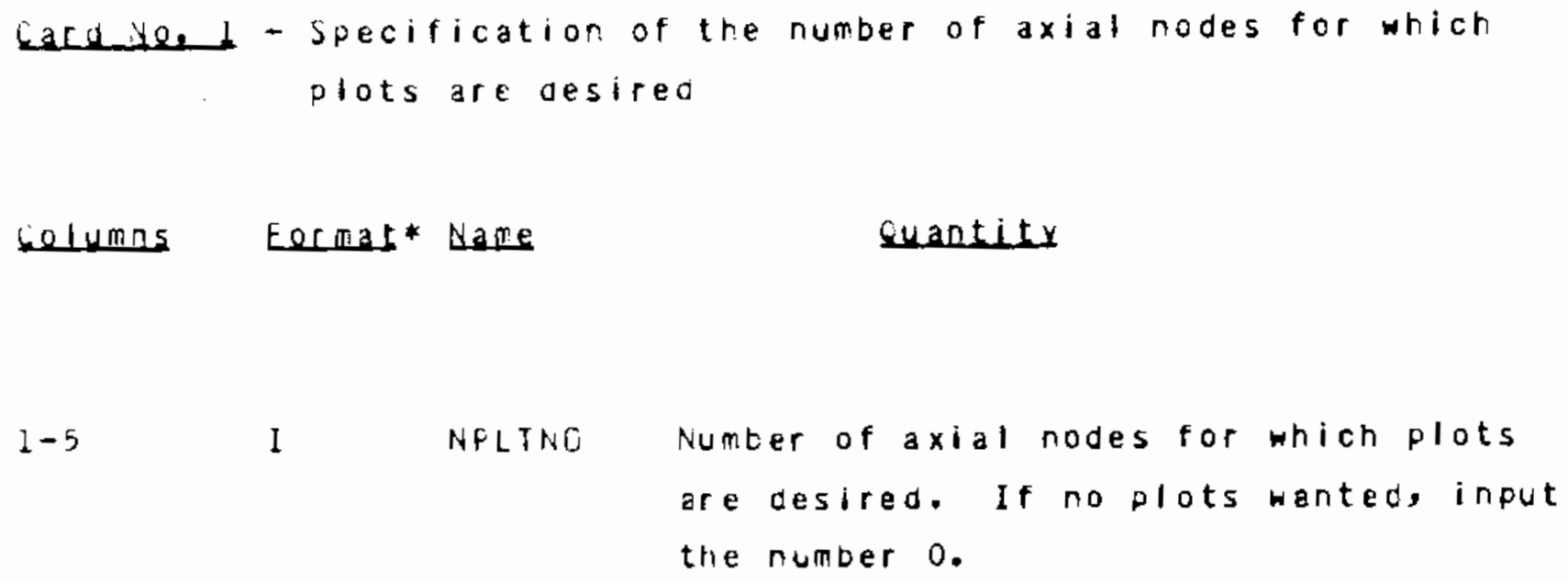

* I integer value, $F=$ floating point value, A a a fona numeric value. 


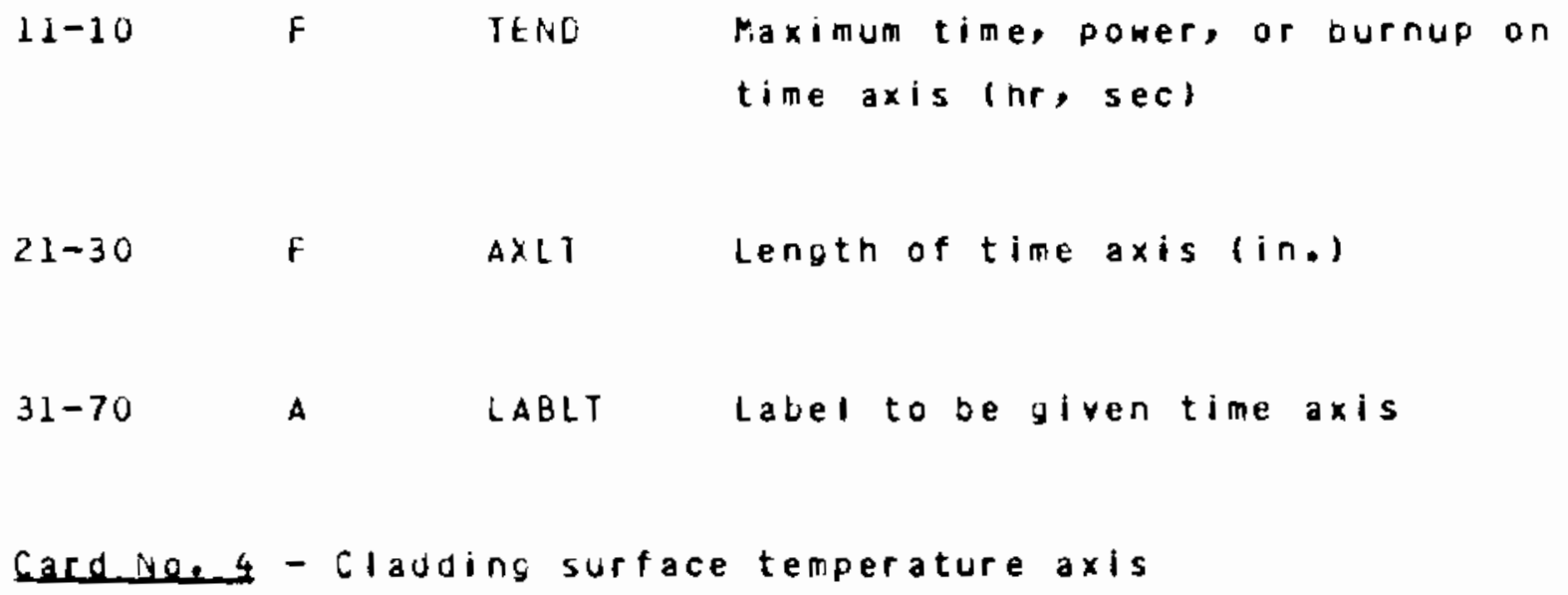

Card Noe 5 - Fuel centerline temperature axis

\section{Columns Earmat Name quantity}

1-10 F TCLMIN Minimum fuel centerline temperature on axis $\left({ }^{\circ} \mathrm{F}, \mathrm{K}\right)$ 

11-20 f TCLMAX Maximum fuel centerline temperature on axis $\left({ }^{\circ} \mathrm{F}, \mathrm{K}\right)$
21-30 F AXLTMP Length of centerline temperature axis $(\mathrm{in} \cdot)$
31-70 A LABLTM Label to be given centerline temperature axis
Gardno, te - Gas pressure axis

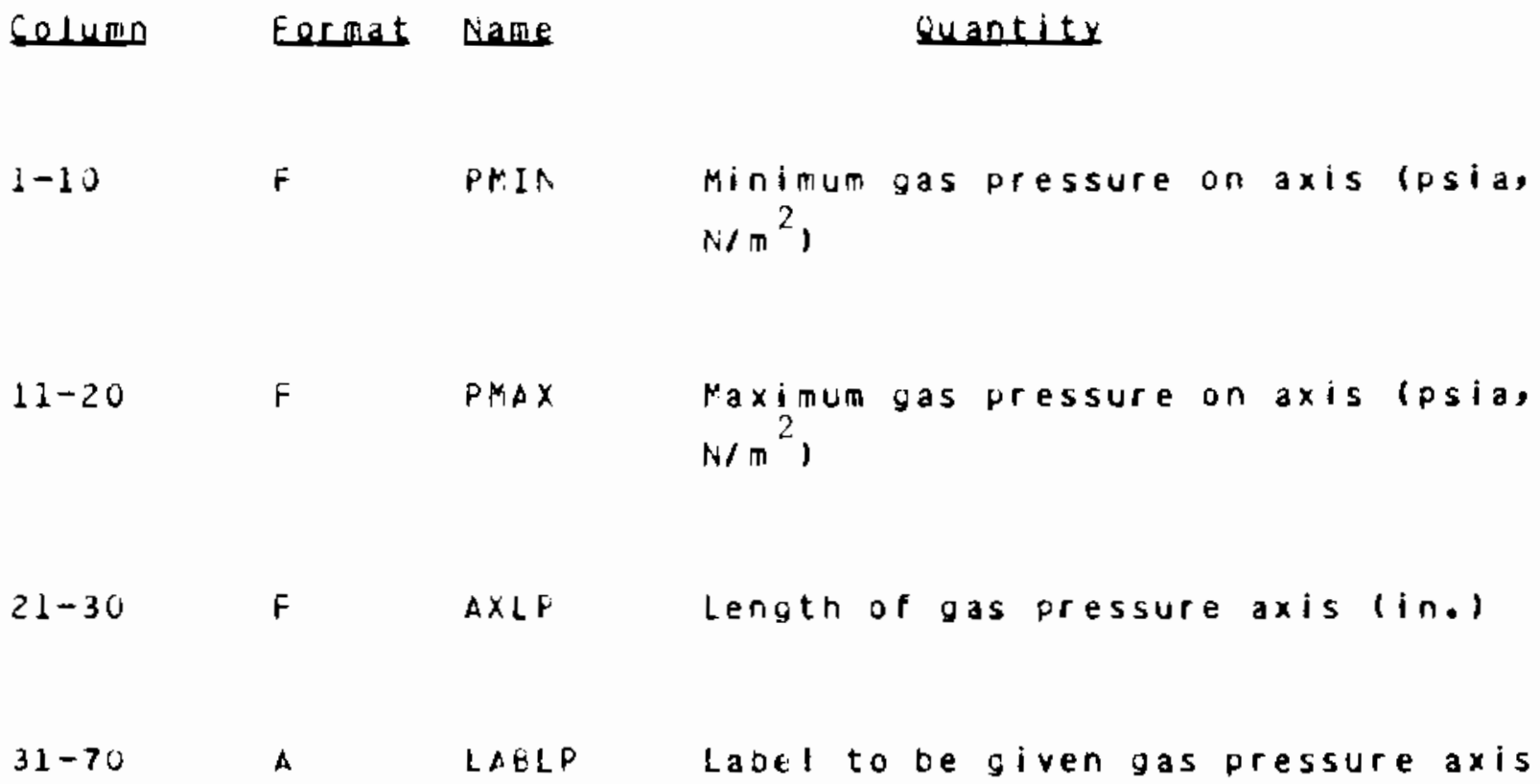

Sarg voe 7 - Cladaing noop strain axis

1-10 F EPSMIN Minimum cladding hoop strain on axis (dimensiontess)

11-20 F EPSMAX Maximum cladoing hoop strain on axis 
(dimensionless)
$21-30$
$\mathrm{F}$
AXLEOS
Lensth of
cladoing hoop strain axis (in.)
$31-70$
A
LAELE
Label to be given cladding hoop strain ax is
Gacdpo. z - tuel axial cisplacenient axis

\begin{tabular}{|c|c|c|c|c|}
\hline celumns & Eormat & Nanie & Guantity & \\
\hline$i-10$ & $F$ & $U Z F+\perp N$ & $\begin{array}{l}\text { Minimum fuel axial displacement on } \\
\text { (ft,m) }\end{array}$ & axis \\
\hline $11-20$ & $\mathrm{~F}$ & UZFMAX & $\begin{array}{l}\text { raximum fuel axial displacement cn } \\
(f t, m)\end{array}$ & axis \\
\hline $21-30$ & $F$ & $\triangle X L U Z F$ & $\begin{array}{l}\text { Length of fuel axial oisplacement } \\
\text { linl. }\end{array}$ & axis \\
\hline $31-70$ & $A$ & LAELUF & $\begin{array}{l}\text { Label to be given fuel axial } \\
\text { aisplacement axis }\end{array}$ & \\
\hline
\end{tabular}
Cacd Nee g - Claddins axial oisplacement axis
columns Eormat same guantity
1-10 F UZCMIN Minimum cladding axial 
displacement on the axis $(f t, m)$
$11-20$
$F$
$U Z C M A X$
Maximum cladding axial displacement on axis $(\mathrm{ft}, \mathrm{nt})$
$21-30$
$\mathrm{F}$
$A X L U Z C$
Length of cladoing axial aisplacenent axis (in.)
$31-70$
A
LABLUC
Label to be given cladoing axial
cisplacement axis

Carciven 10 - Fuel roo pomer axis

Gelumis Eormat Dare

vuantity

$1-10$

$F$

PF.IT.

Minimum linear fuel roc power on axis $(\mathrm{kin} / \mathrm{ft}, \mathrm{kw} / \mathrm{m})$
$11-20$
$F \quad P A A X$
Maximum linear fuel roc power on axis $(k w / f t, k w / m)$
$21-30$
F PLEN
Length of 1 inear fuel rod power exis (in.)
$31-70$
A
PLAEL
Label to be given linear fuel roo poker axis

Cacd Nee_d - Fuel surface temperature axis 


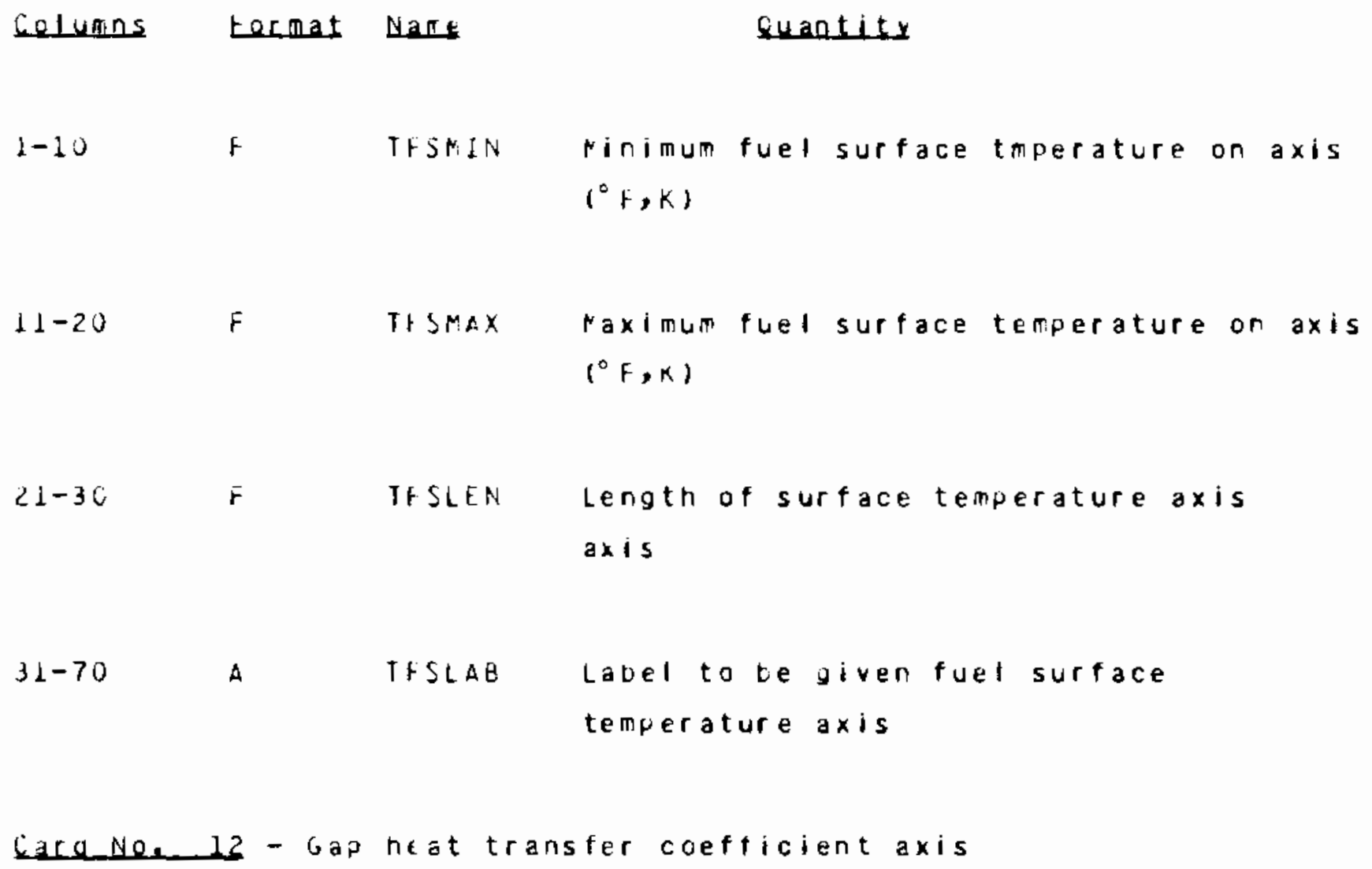




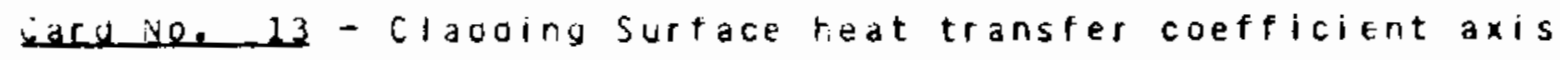

\begin{tabular}{|c|c|c|c|}
\hline Gelumas & Locmat & Manse & Quantity \\
\hline $1-10$ & $F$ & HSHIN & $\begin{array}{l}\text { Minimun surface heat transfer } \\
\text { coefficient on axis (Btu/rr. }{ }^{\circ} F . f t^{2} \text {, } \\
\left.k, m^{2} . k\right)\end{array}$ \\
\hline $11-20$ & $F$ & $\operatorname{HSMAX}$ & $\begin{array}{l}\text { Maximum surface heat transfer } \\
\text { coefficient on axis (Btulhr. }{ }^{\circ}+\mathrm{ft}^{2} \text {, } \\
\left.\mathrm{m}^{2}, \mathrm{k}\right)\end{array}$ \\
\hline $21-30$ & $F$ & HSLEN & $\begin{array}{l}\text { Lengti of surface neat transfer } \\
\text { coefficient axis cinel }\end{array}$ \\
\hline $31-70$ & $A$ & $M S L A B$ & $\begin{array}{l}\text { Label to be given surface heat transfer } \\
\text { coefficient axis }\end{array}$ \\
\hline
\end{tabular}

bargiver 14 - average cladoing terperature axis

Gelumes Eormat Name suantity

1-10 $\quad$ TAMIN Minimum average cladoino temperature on axis $\left({ }^{\circ} \mathrm{F}, \mathrm{K}\right)$

11-20 F tamaX raximum average cladding temperature on axis $\left({ }^{\circ} \mathrm{F}, \mathrm{K}\right)$

21-30 F TALEN Length of average cladoing temperature axis (in) 

$31-70$
A
TALABL
Label to te yiven average cladding temperature axis
Gargne._25 - Zircaloy-Gxide thickness axis

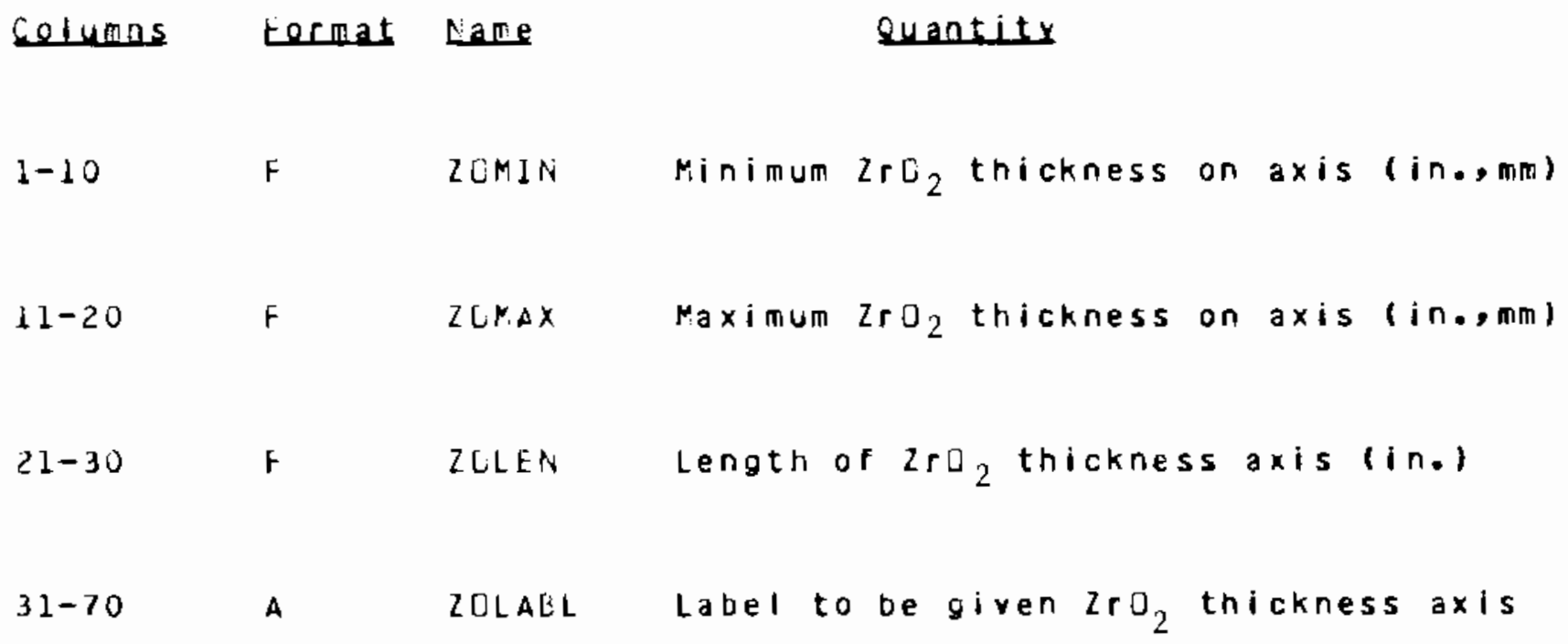

Caca Noe_16 - Helium niole fraction axis

\begin{tabular}{|c|c|c|c|}
\hline Sotumas & Eormat & Lame & Quantity \\
\hline $1-10$ & $\mathrm{~F}$ & MFMIN & Minimum helium mole fraction on axis \\
\hline $11-20$ & $f$ & MIF MAX & Maximum helium mole fraction on axis \\
\hline $21-30$ & r & MFLEN & $\begin{array}{l}\text { Length of helium mole fraction axis } \\
\text { lin.l }\end{array}$ \\
\hline $31-70$ & A & MFLABL & $\begin{array}{l}\text { Label to be given helium mole fraction } \\
\text { axis }\end{array}$ \\
\hline
\end{tabular}




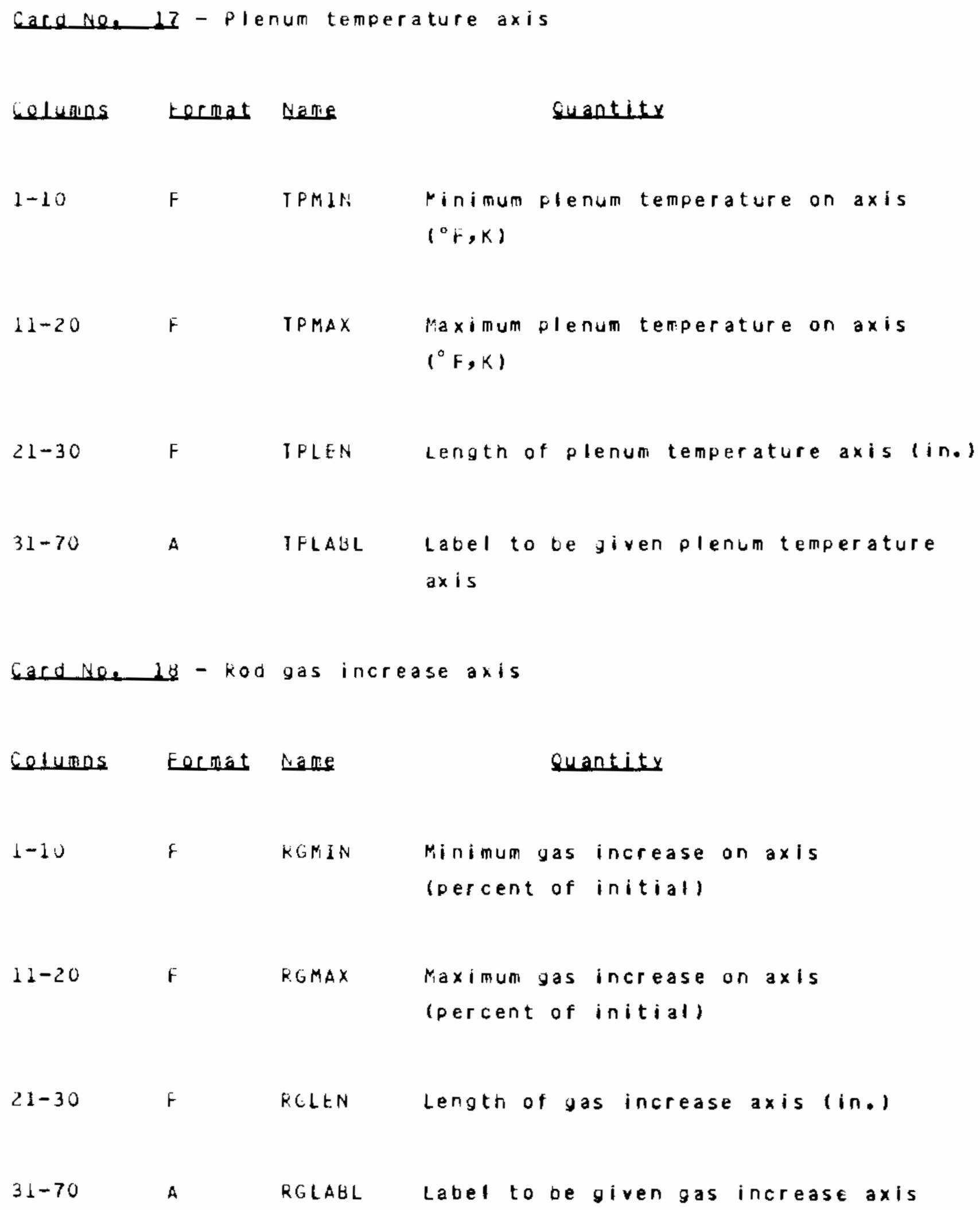




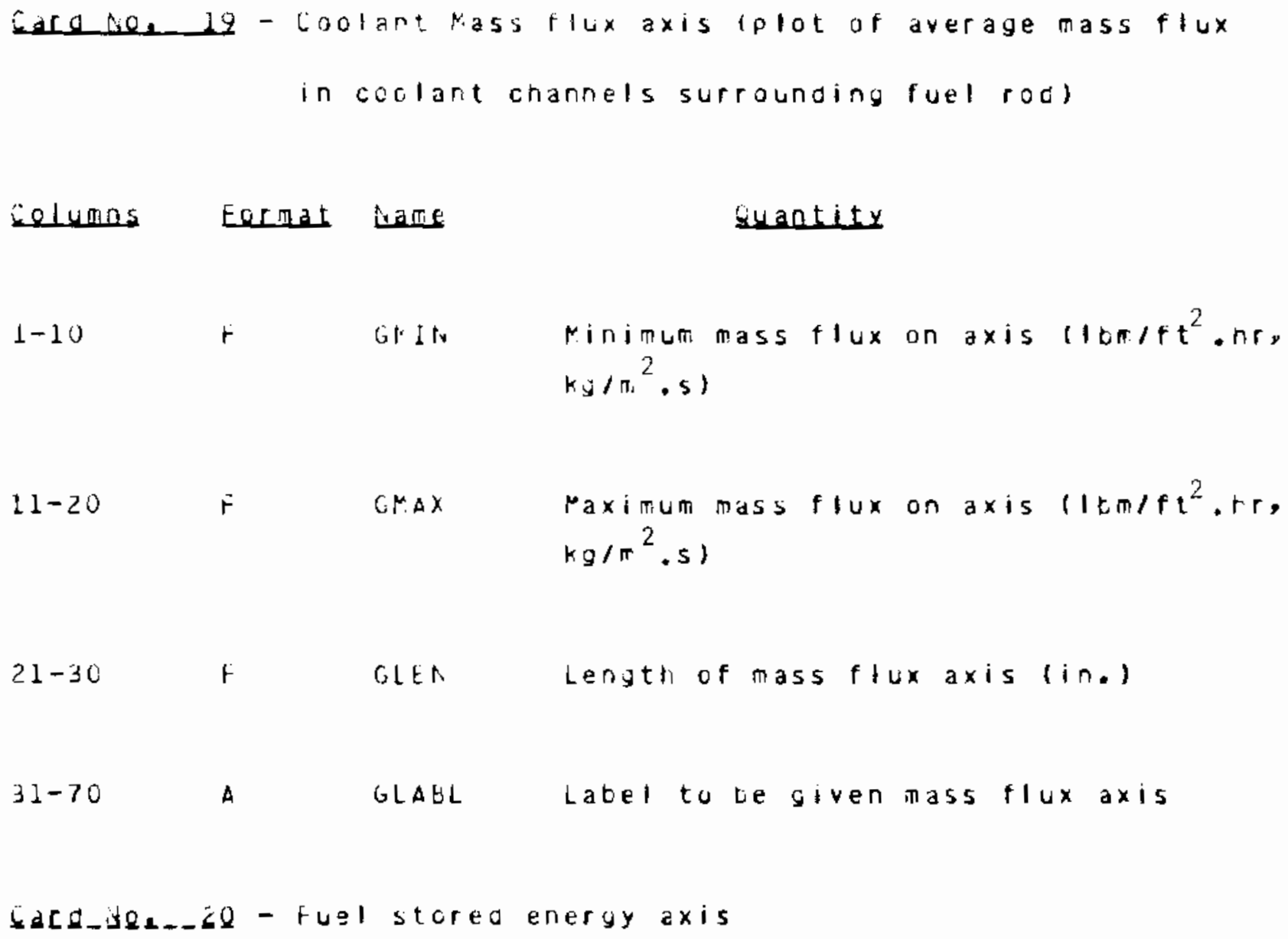




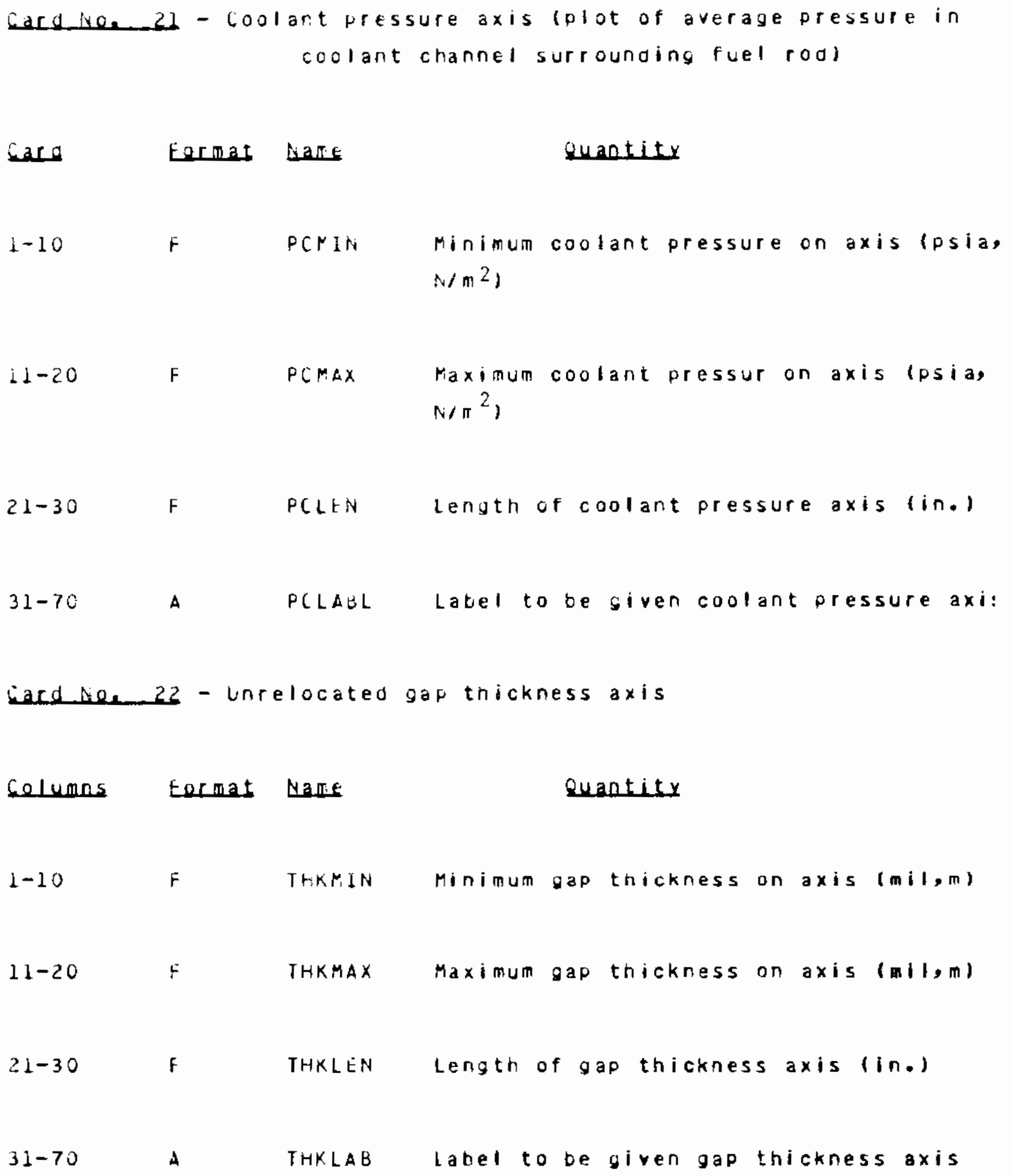


Gacdne 23 - tulk coolant temperature axis

Celunas Eocmat Name Quantity

1-10 F TEMIN Minimumbulk temperature cn axis $\left({ }^{\circ} \mathrm{F}, \mathrm{K}\right)$

11-20 F TEMAX Maximum bulk temperature on axis $\left({ }^{\circ} \mathrm{F}, \mathrm{K}\right)$

21-30 F TBLEN Length of butk temperature axis (in.)

31-70 A TELAB Label to be given bulk temperature axis

3. OUTPUT OESCRIPTION

The output from FRAPCON-2 consists of several discrete sections. Each section will be described below in the order the user mouto encounter it.

The first page is an exact listing of the cards the program reads as data (figure $A-1$ ). The wRITE statements which create this output are in sliBRQUTINE ECHOL.

If the uncertainty option $f 1 a g$ is set to "1", the next section of output wil present uncertainty analysis output (Figure $A-2$ ).

The next output section to appear in the printout is the NAMELIST FRPCN with the values for those variables (Figure a-3). This information is created by the WRITE statements in subroutine SETUP. 


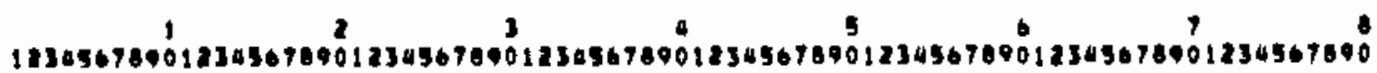

IFA-A32, 由OD 1

SFAPCN

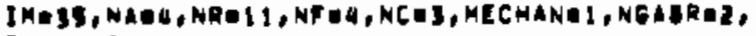

Im 10,

IM 12 ,

AEND

C) RPCON

RENTE D104.0, TSINT 3092.0, FLUX M.1E1T,

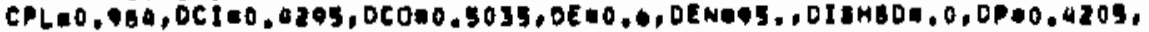

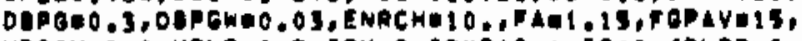

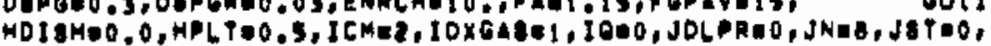

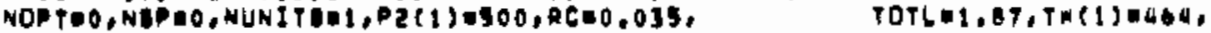

IPLANT:-1,

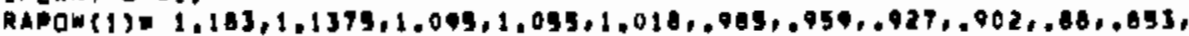

of (1) $190,580,0.67,0.701,0.062,0,917,0,068,0,906,1,0$,

Qmpr(10; o, $13,7,6+12,8,3 * 13,7,12,4,3+11,12 * 12,2,3 * 11,4,2+10,2,11,2$,

Ompres $\bullet 1,12,2,11,2,10,2,9,2,2,8,4,0,4,10,4,8111,4$,

TIME (22) - 125,2,147,.108,1180,,210,.230,,230,2,250,,270,1270,2,270,4,

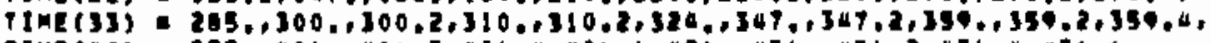

PIME(40) 375,,406,,400,2,406,4,400,0,421,,436,,436,2,436,4,436,0,

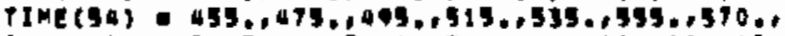

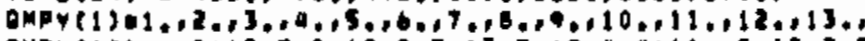

gmpy (19): $0.13,7,8+12,8,5 * 13,7,12,4,4 * 11,12 * 12,2,5+11,4,2=10,2,11,2$,

ancy (30) $12=12,2,11,2,10,2,0,2,4 * 0,4,0,4,10,4,10411,4$,

TIme (1) $0,075,0150,0.225,0,300,0,375,0,450,0,325,0,600,0.075$,

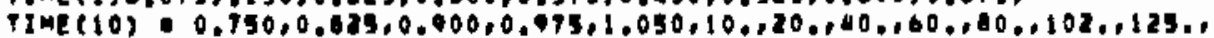

TIME (22) $175,2,140,1155,1170,1105,1200,1215,1230,1230,2,240,1250, \ldots$

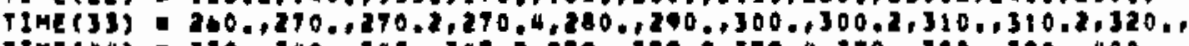

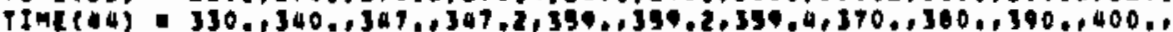

TIME(35) $400,, 406,2,406,4,406,6,406,8,416,, 426, .436,1436,2,436,4$,

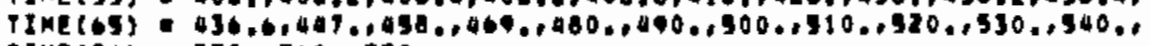

TIME(T) $150,1500,1570.1$

$x(1) 00,0,0,25,0,541,0,033,1,241,1,350,1,098,1,01$,

JOLPQ 1 ,

SEND

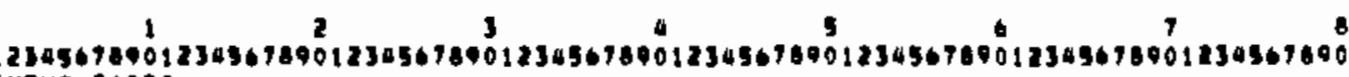
36 INPUT CARDS

Fig. A-1. Card 11st generated by ECHOl. 


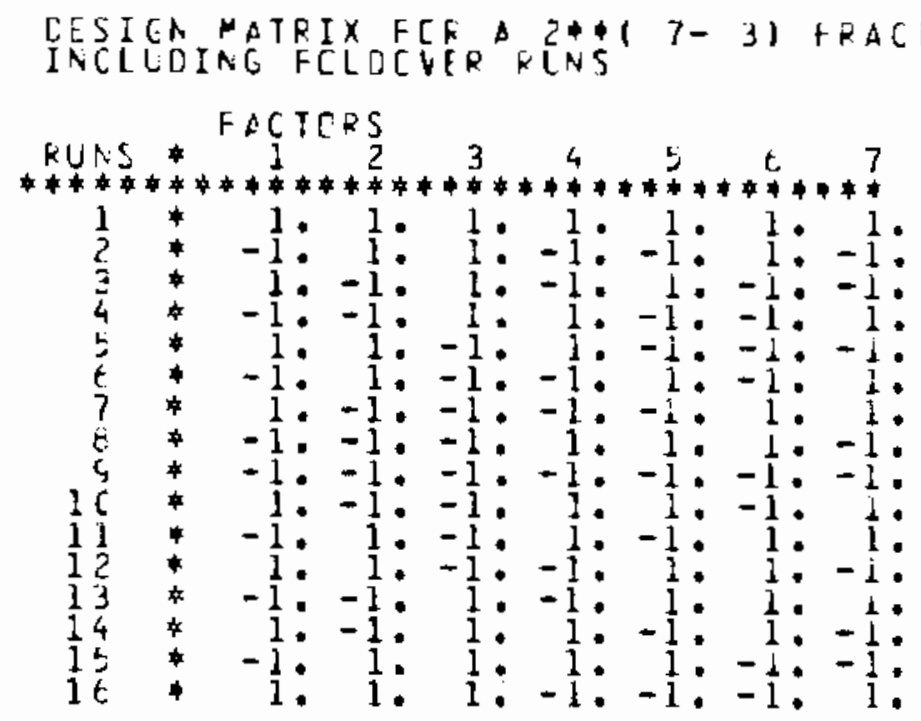

CCNFCUADINE ARRAY

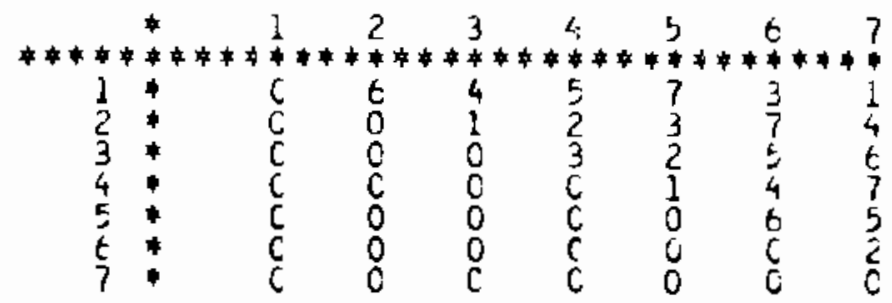

Figure A-2 Uncertainty printout. 


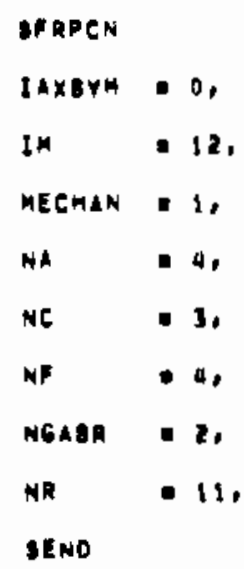

Figure A-3. Namelist FRPCN.

UIFIRA 3017 UILAST 16822

THE NUMBER OF ARRAY is 279

THE LENGTH OF THE AOARRAY IS IOAB2

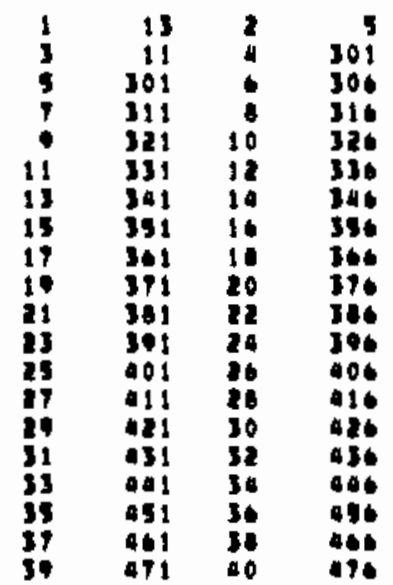

Figure A-4. Dynamic dimenstontng output. 
SUBRUUTINE PUINTR frints a column of numbers that follow the sEND from NAMELIST FRPCN. These numbers designate array locations as calculated by the dynamic oimensioning capability of the code ifigure $A-4)$.

The NAMELIST FRPCON is the next section of output (figure a-5). This will be followec by any diagnostic messages generated by the data checking routine in the code, SUARQUTINE CHECK.

SUBROUTINE PRINTl generates the table of physical dimensions which appear after the diagriostics messages, or the SEND of NAMELIST FRPCLN (Figure A-6). The tieading at the top of the page is generated Dy SUBROUTINE PGHEAC. It contains the FRAPCON code version number, the MATPRo version number, the run date, an option indicator lsee Jable A-3) and the page number for future reference. The title also appears in this pase heading.

The time dependent variables which may be input by the user are generated by the PkINTl subroutine and are listed on page 2 of the output (Figure A-7). Plot information will also be output here.

Page 3 which is generated oy SUBROUTINE AXHEF, gives information on how the code interpreted the user's input with respect to axial and radial power profiles. Initial fill gas composition is also output. The zero poner cladding axial expansion is provided for data comparison purposes (figure $(-8)$ ). 


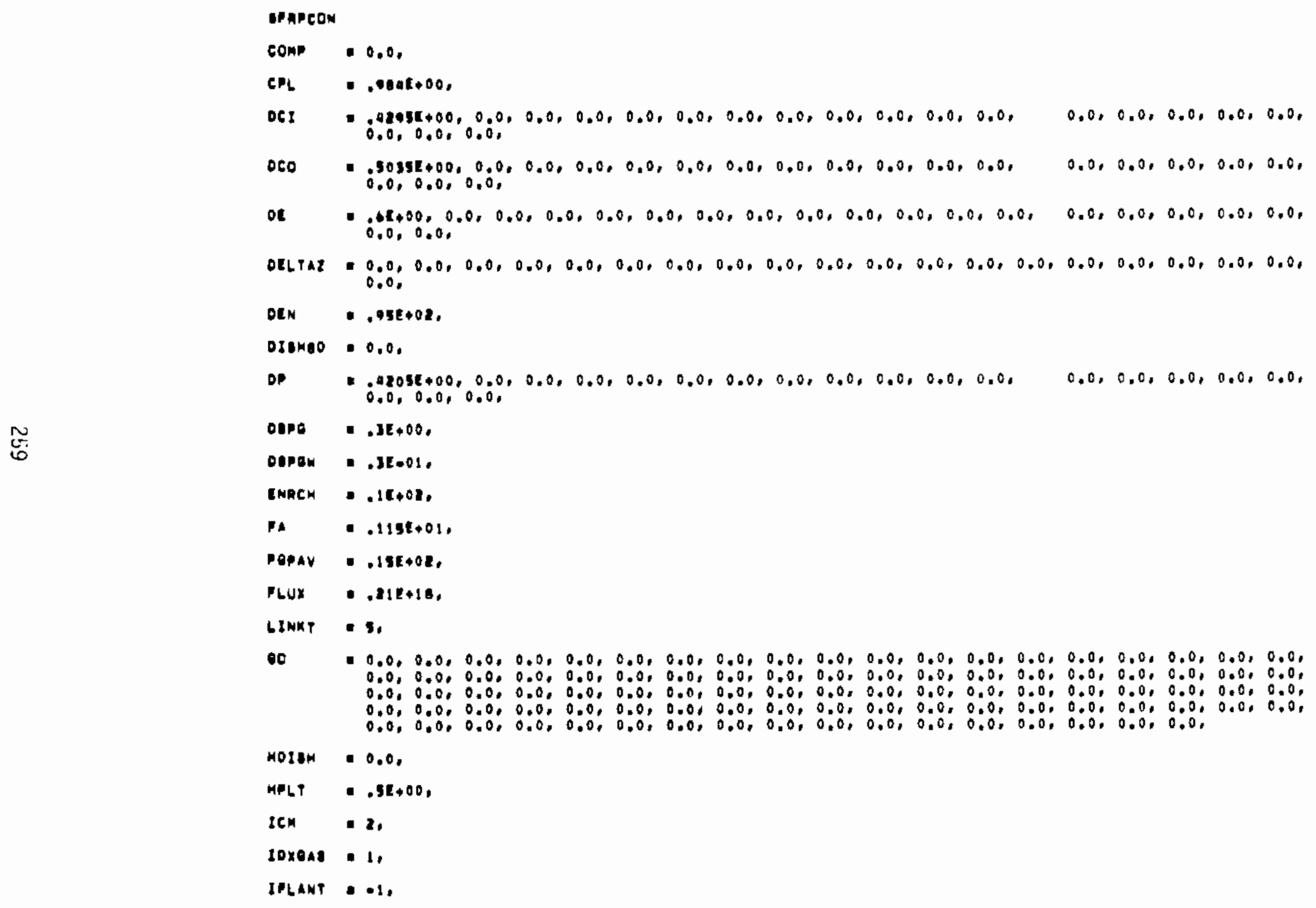

Figure A-5. Namelist FRPCON, 


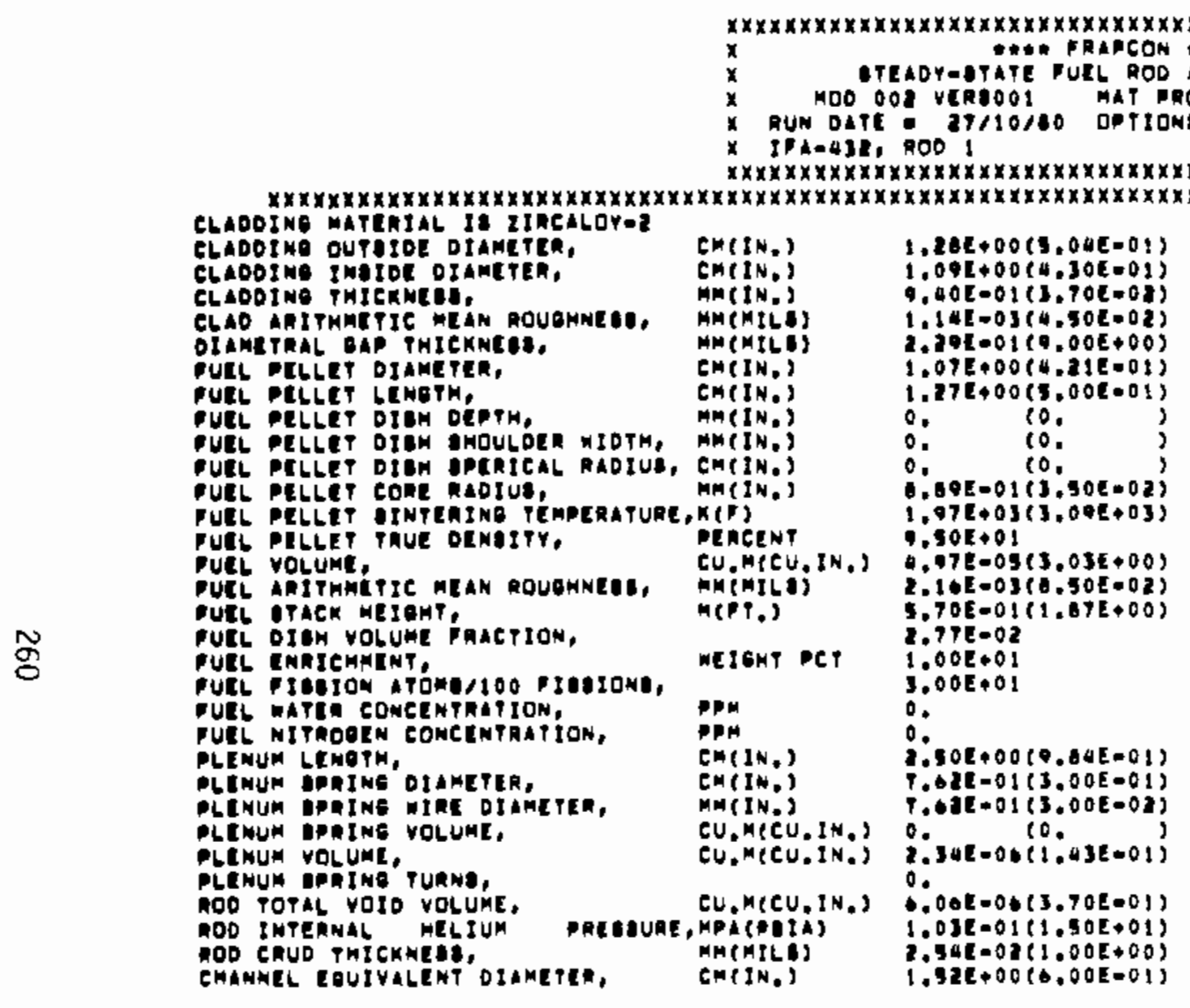

Figure A-6. Initial dimensions. 
example:

$$
\begin{aligned}
& \text { UPTIONS } 7221000000 \\
& \text { number } 123456789 \\
& \text { tach number is cesigned to signify a model option. }
\end{aligned}
$$

The first number indicates the radial power profile option choice

=1, LASER generated: Phk uranium enriched

=2, LASER generatec: BhR uranium enricned

$=3$, LASER generated: PhR plutonium enriched

$=4$, LASER generateg: bih plutonium enriched

$=b$, Lser supflied radial poner profile

$=6$, FluxD computed profile with zero enrichment

=7, Fluxo conputeo profile nith enrichment greater than zero

The second number incicates the gas release option choice:

=1, Beyer-Hann with NRC high burnup correction

$=2$, Eeyer-Harin without NRC high burnup correction

$=3$, ANS 5.4

$=4$, booth diffusion

$=5$, GRASS Or FAST-GRASS

The thira number indicates the mechanics option choice.

$=1$, PELET/RADIAL

$=2, \quad F K A C A S-1$

3, FRACAS-1I

The renainder of the numbers are not presently used. 


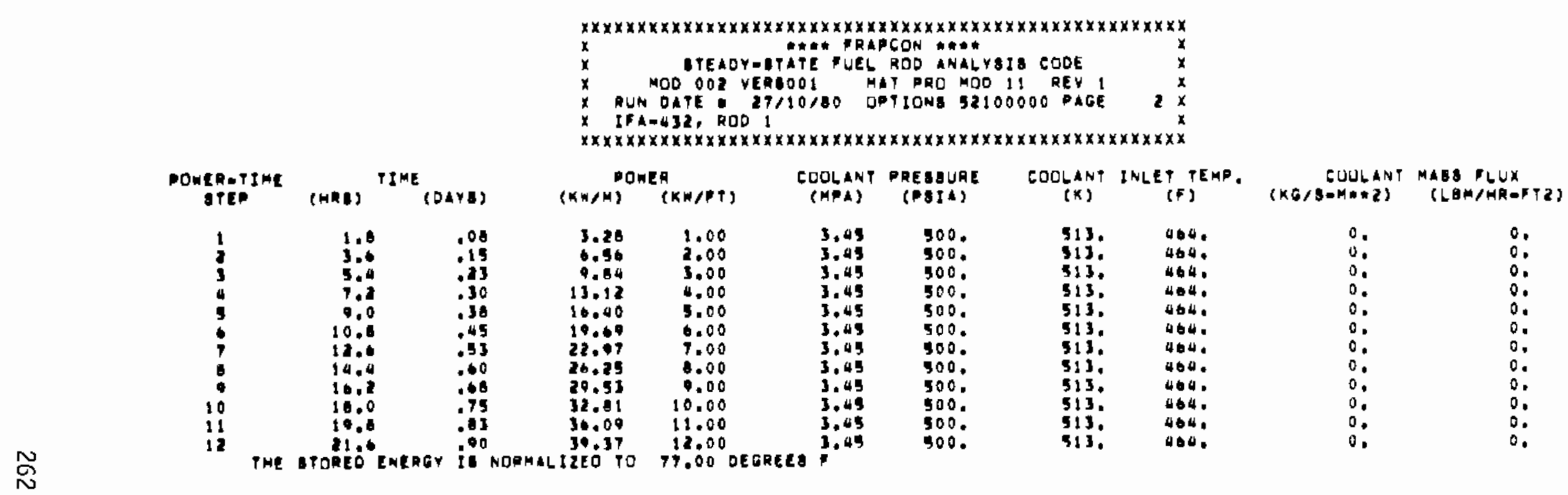

PLOTS NOT REQULATEO 


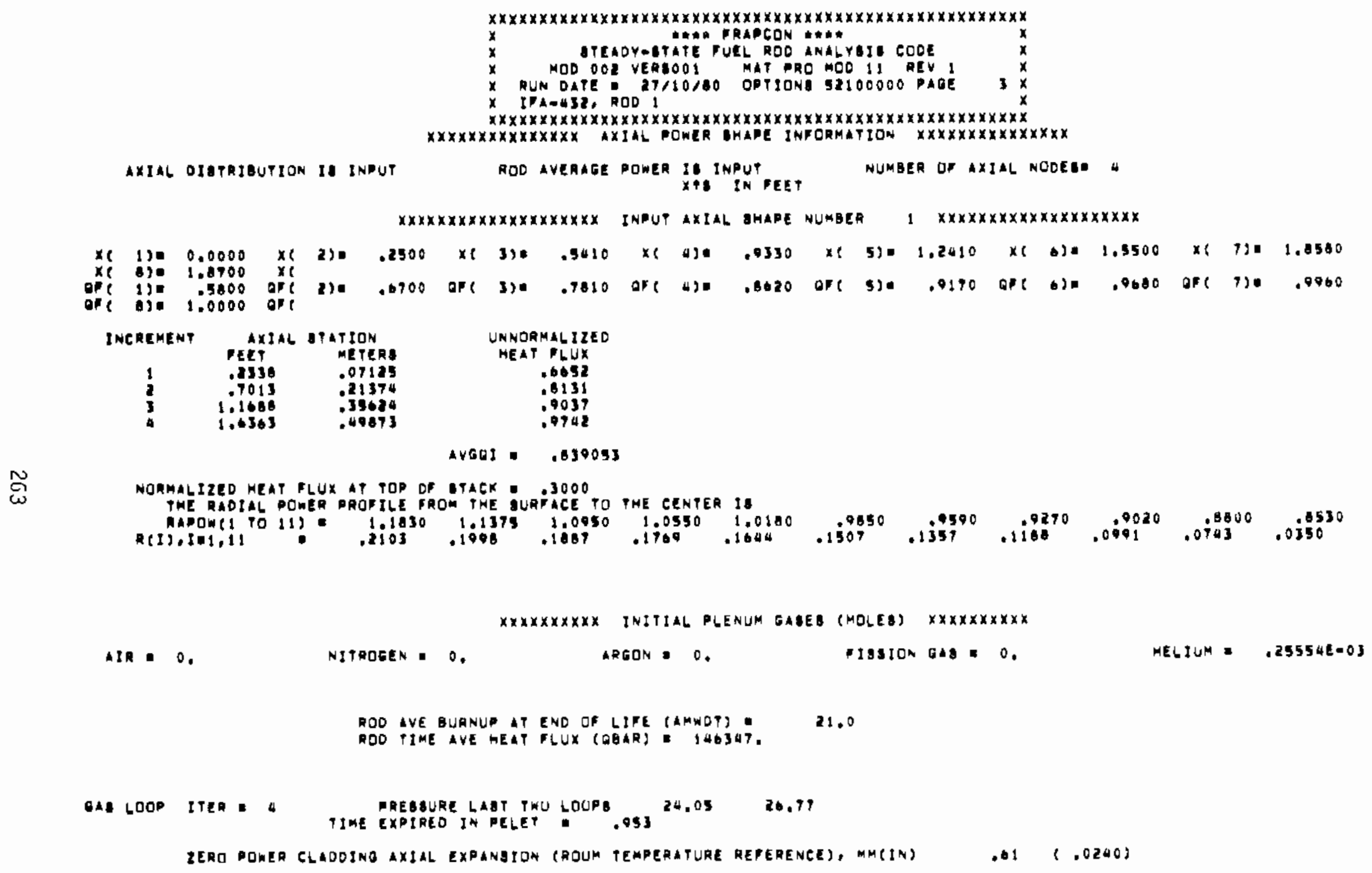

Figure A-8. Power profiles. 
Starting itn Pagt 4 the output is generated by the code as it runs the simulation. For each time step, there will be a page describing the state of the fuel roa for each axial region (figure a9.), a sumbry pase (Figure A-10). The output for each axial node contains such things as fuet roo linear heat rating, ournup, time in reactor, temperatures and dimensions, jas conouciivity, gap size and conductance, stresses and strains in the cladoing, and pellet-claoding interfacial fressure. If the relet option is used, the effective fuel elastic moduli and tre calculatec fuel thermal concuctivity cegradion factor are also output.

The suminary fage includes information on the volumes and temperatures used in the gas fressure calculation, fission gas release and a complete sumary for aft tre axial nodes. This sumary for each axial node includes claduing stresses, total strains and permanent strains, fuel centerline temperature, fuel average temperature, cladoins temperature, coolant temperature and the stored energy in the fuel. rinaliy, the cladding axial expansion is provided.

If the Als 5.4 fission gas release model is used, a table ano plot of tission gas relcase fraction versus half-life is output for the shorter nalf-life "radioactive" gases (figure A-11). This fage precedes the other outfut pages for that time step.

ror the final time step, final summary pages are provided (rigures a-12, A-13). Inis summary contains information of interest nith resfect to pean poner segment values for each time step.

4. Jug cuntEgl language feik cle cyber

The job control caras necessary to run rRAPCON-2 on COC CYBER computers are listed below: 


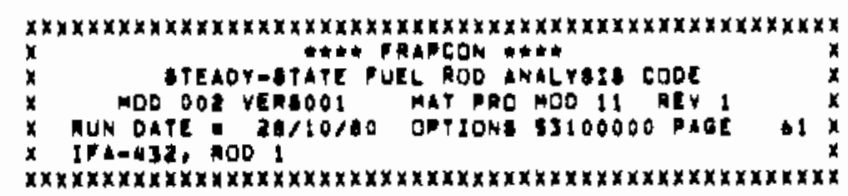

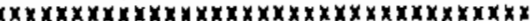

AXIAL REgION NUMBEA " DOWERTITE BTE 29

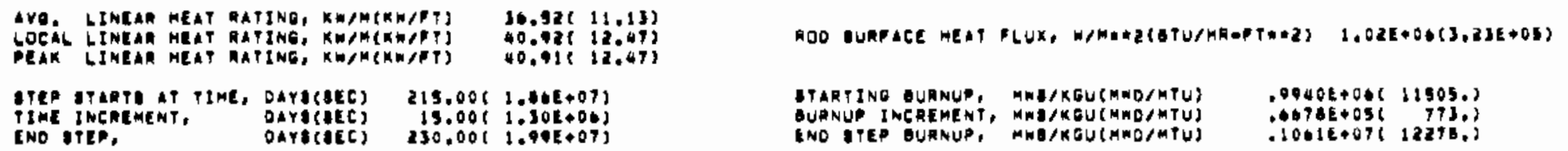

ENO DTEPEMEN, DAYS(EEC) $13.00(1.30 E+06)$

RAOTAL TEMPEATTURE DIATRIBUTION

PAOIUJ, CH(IN) TEMP, K(F)

\begin{tabular}{|c|c|c|}
\hline 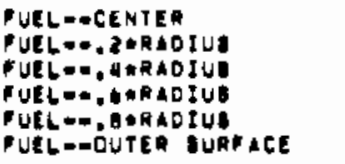 & $\begin{array}{l}.00096 \\
.10031 \\
.21056 \\
.32701 \\
.43716 \\
.50036\end{array}$ & $\begin{array}{l}.03501 \\
.04301 \\
: 08609 \\
.12919 \\
.1724 \\
.21519\end{array}$ \\
\hline $\begin{array}{l}\text { CLADOING INNER EURFACE } \\
\text { CLADOINE OUTER OURFACE }\end{array}$ & $\begin{array}{l}.94001 \\
: 04241\end{array}$ & $\begin{array}{l}.23000 \\
.25200\end{array}$ \\
\hline OXIOE UURTACE & .04256 & .25301 \\
\hline
\end{tabular}

COOLANT TEMPERATURE

MAOIAL GEOMETRY ENAMGES

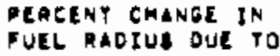

OENGLFTCATION THELLING

DERTMAL EXPANBION

PUTAL

ect change in cladoing AAOZUS OUE TO

meamanent

COVERAGL

TDTAL
INTERFAG CONOITIONB

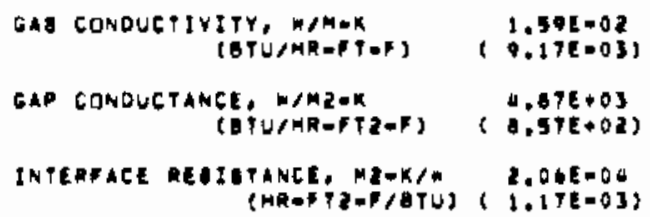

FRATIZON CONDUCTANCE DUE TO

Ga CONOUCTANCE

COMTACT

.011
INTERTACE PRT

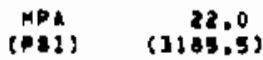

$2 ? 23,(0441$.

2937.1409 .3

$2097,13279 . ;$

1447.12104 .5

$579,5503 . ?$
$324 .,(465 . ?$

513.8404 .1

$513,(464$,

\section{BTREBgEB, MPA(KSI) BTRAINE, PCT}

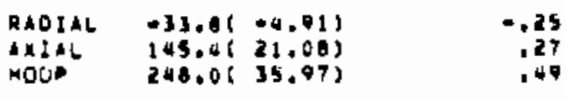

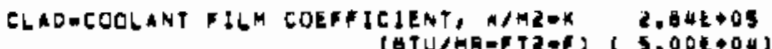

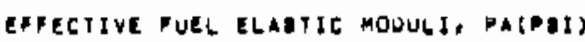

RAOIAL $3, J 5 E+0 Q(4,0 \otimes E+O 5)$

wel TmERmal CONOUETIYITY

Figure A-9. Output page for one axial node. 


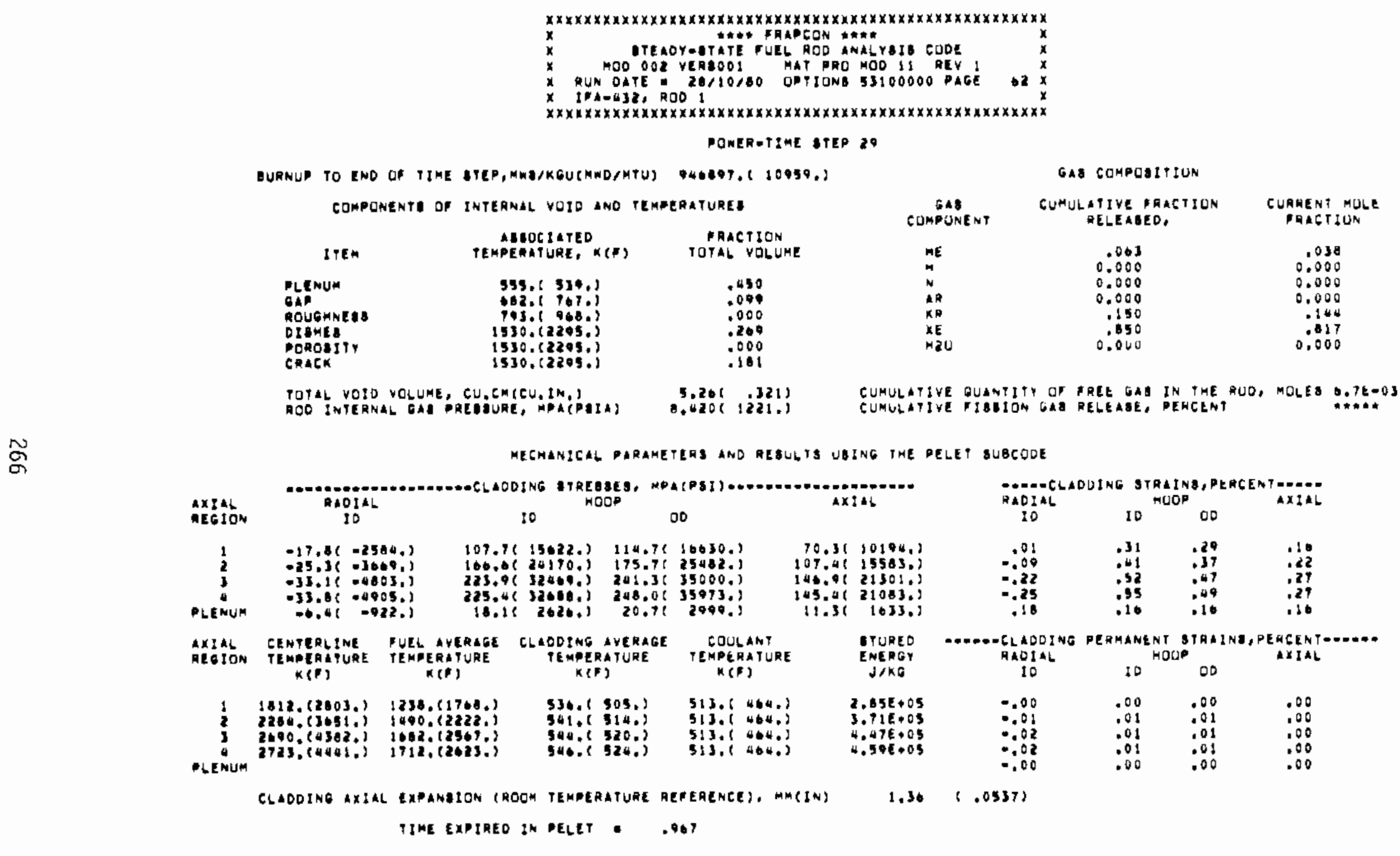

Figure A-10. Output page for one time step. 
FRAGTION RADIDAETIYE GABES RELEABEO

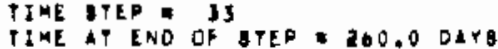

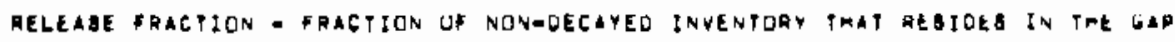

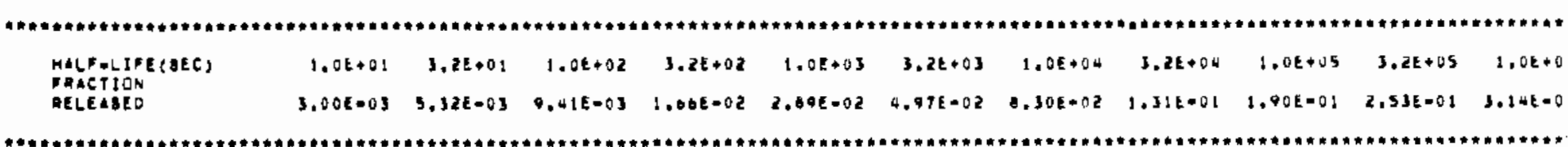

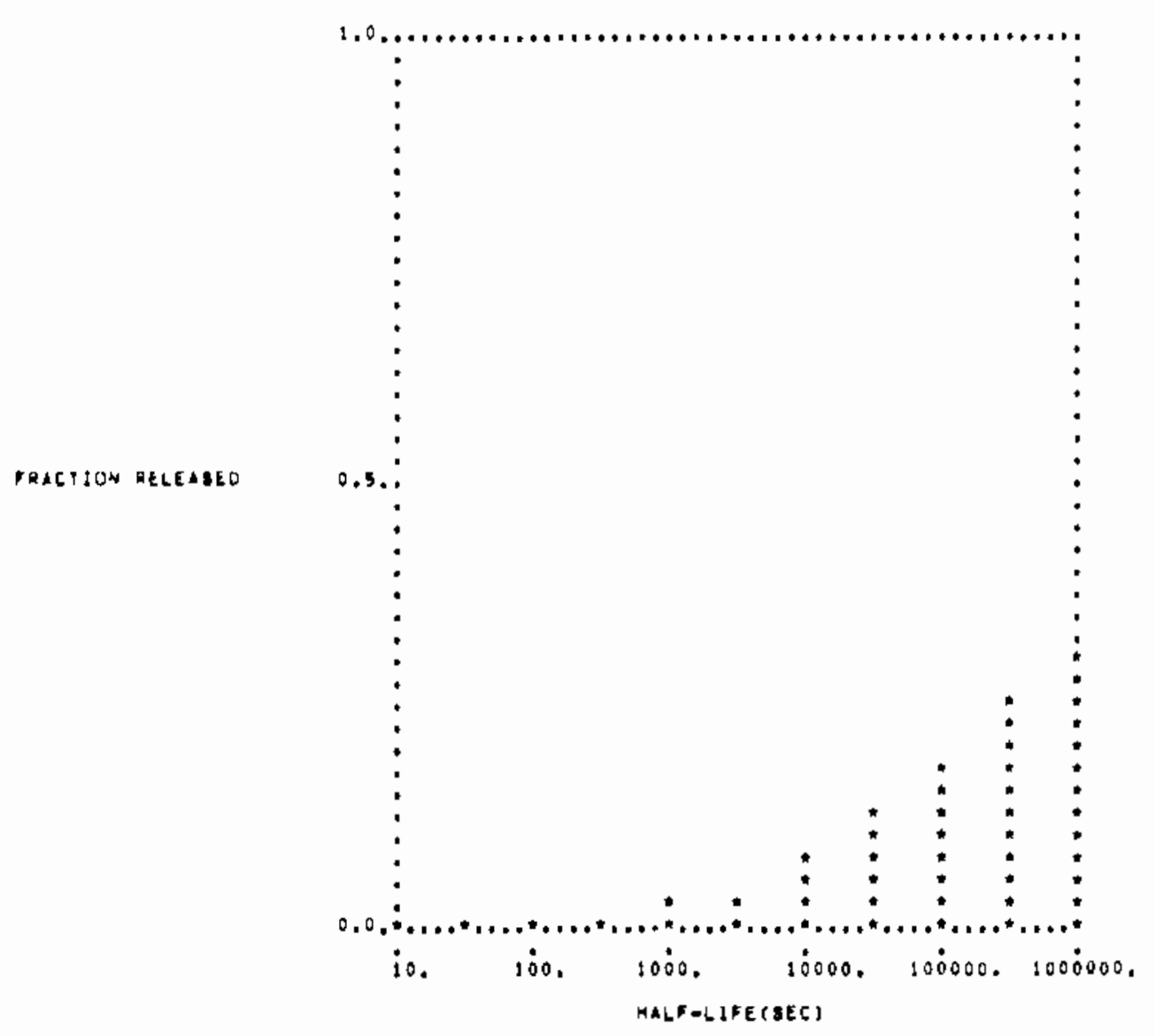

Figure A-11. ANS-5.4 radioactive (short-1ived) fission gas release. 


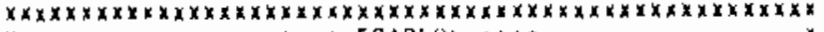

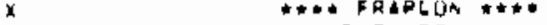 \\ BTEADYGTTEE FUEL ROD RNALYSIS COUE

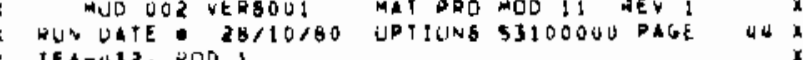 $164=432,2001$}

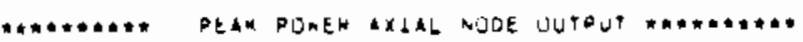

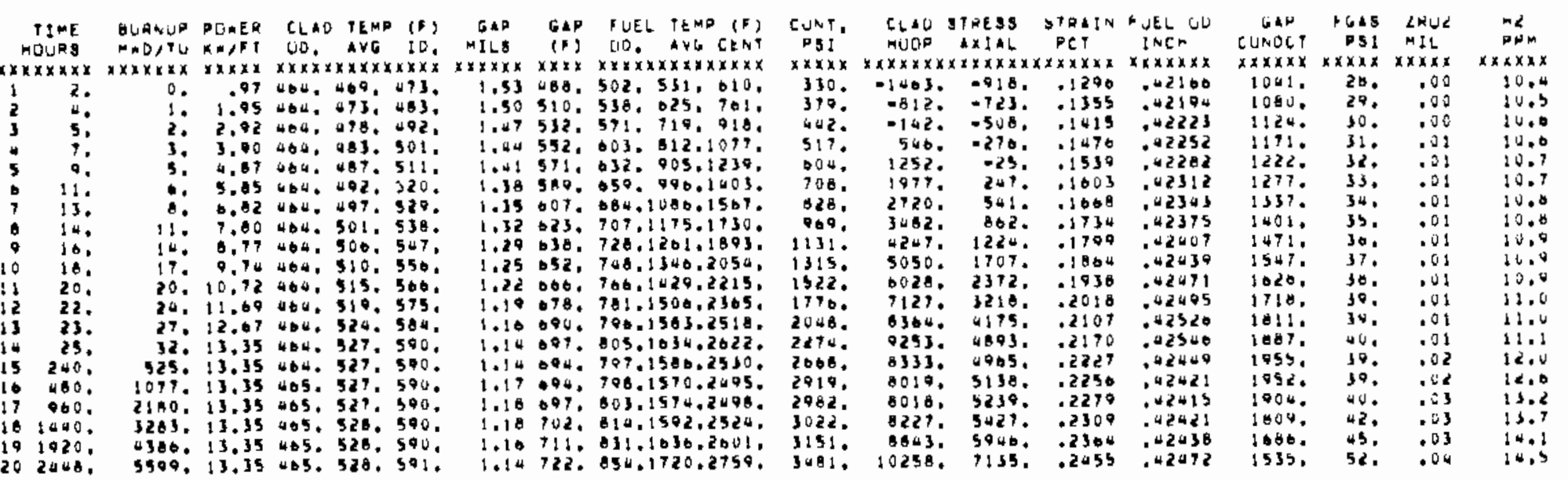




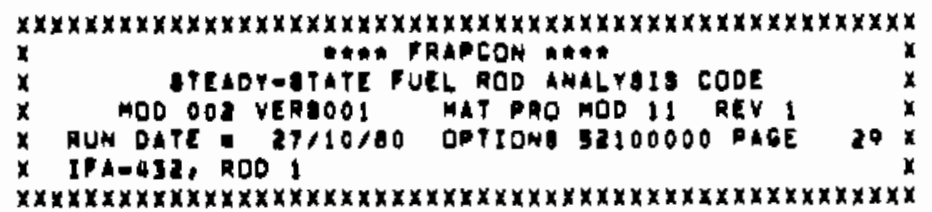

END OF LITE ITRAIN RANGE (PEACENT) - .00OB

Fiagion gal CUMULATIVE FRAETION RELEABE = ,000744

2ROZ WEIOHT GAIN , (GM/MHEZ) .

Figure A-13. E0L fission gas release fraction. 


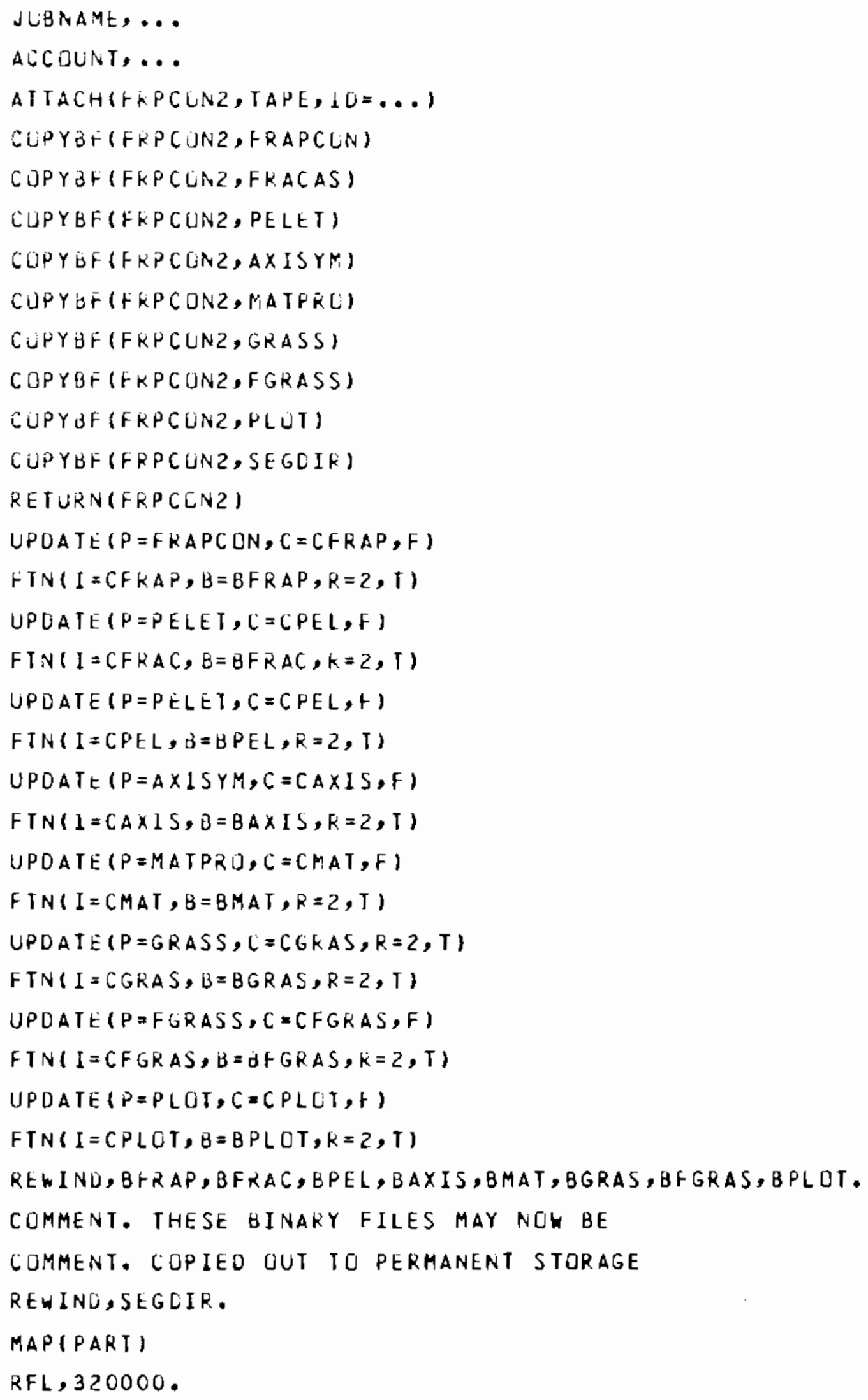


CGMMENT. THE RFL CARO WILL VAKY FKLM MACHINE

COMMENT. TO MACHINE ANL DEPENLING ON THE INPUT

COMMENT. PARAMETERS SPECIFIED

SEGLQAU(I) SEGOIR, B=FKAPGO)

LUAD, UTRAP, BMAT, ...

COMMEIT. BHKAP AND BPAT AKE aLWAYS REQUIRED

CUMMENT. BFRAC IS KEQUIRED FUR FKACAS-II

COMMENT. BPEL IS KEQLIKEL FOR PELET.

CUMMENT. BGRAS IS RECUIKED FLR GRASS.

COMMENT. UFGRAS IS REQUIRED FER FAST/GRASS

COMMENT. BAXIS IS FECUIRED FCK AXISYM

CUMMEVT. BPLUT IS REQUIREO FOR PLOTS

NUSO.

CGMMENT. FRAPGO maY HE COPIEO UUT TO PERMANENT

CUMMENT. STORAGL TL SAVE RELCACING EACH TIME.

FKAPGL, PL $=100000$.

$+t a R$

*EOR

*EQR

*EOR

* E QR

$* E Q R$

*EQR

* EOR

HEUR

JATA STREAM

*EOF

If the different files are copied out to permanent storage as suggested by the comment cards, the sequence of job control cards can be begun with the FRAPGO card in succeeding runs. An RFL cardwill always be needed, however. 
APPENOIX B

MATEKIAL PRLPEKIILS CUKRELATILNS EMPLOYEO BY FRAPCLN-2 
APPENLIX $B$

\section{MATEKIALS PREPEFTIES CURRELATIONS}

\section{EMPLOYEO EY FRAPCON-2}

A materials properties subcode is used to provide the computational subcodes of rkAPCON-2 with gas, fuel, and clacaing materials properties. Those properties used by FRAPCDN from Reference 3 are presented in Table $B-1$.

TaOle b-1. MATERIALS PROPERTIES IN MATPRO USED GY FRAPCON-2 Property

Subroutine

\section{Euel Material properties}

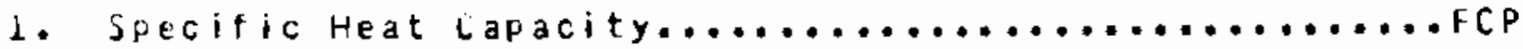

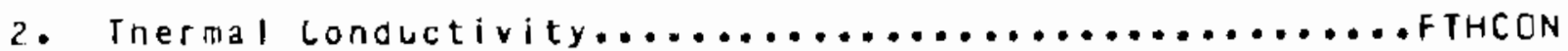

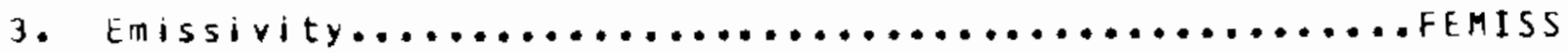

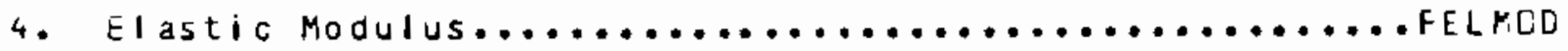

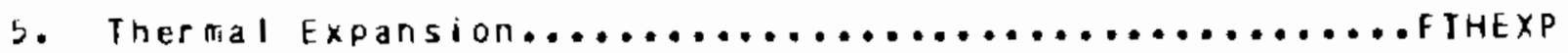

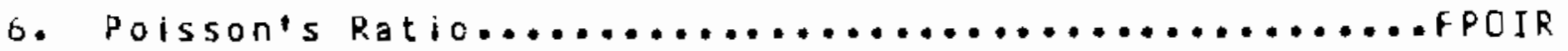

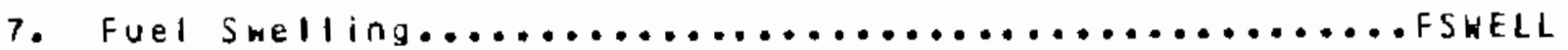

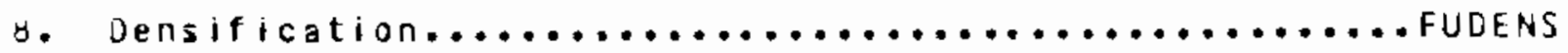

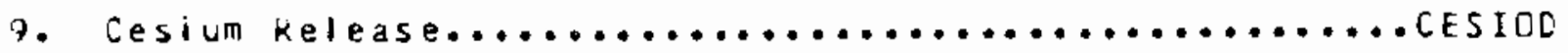

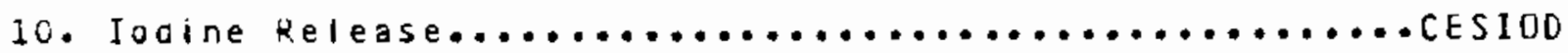


Table $\theta-1$ (continued).

\section{Cladding Material Proferties}

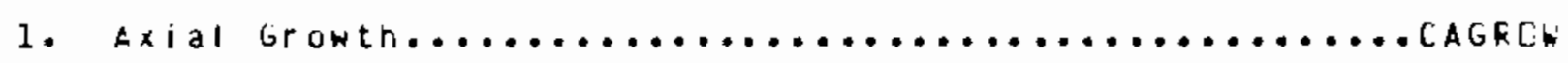

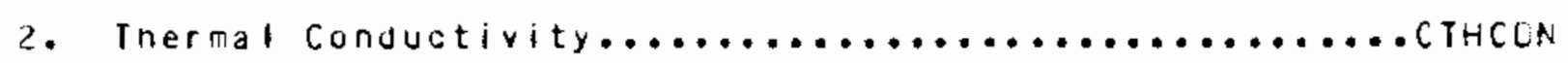

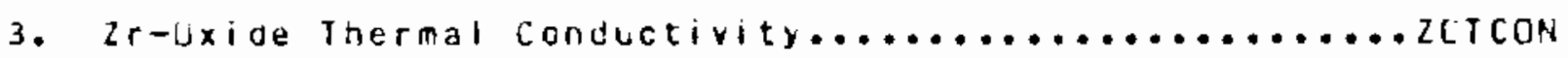

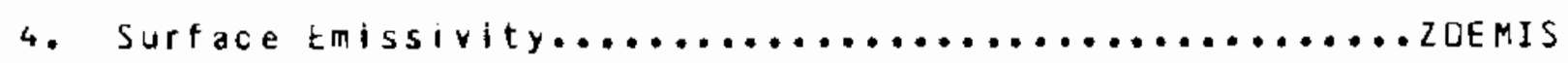

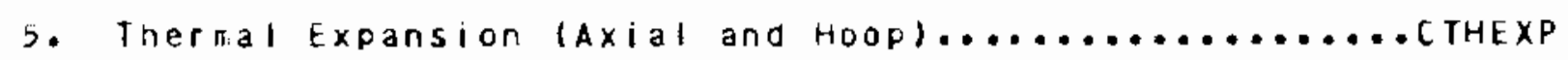

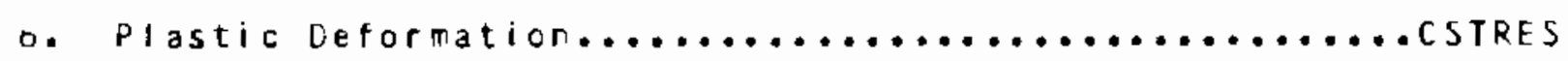

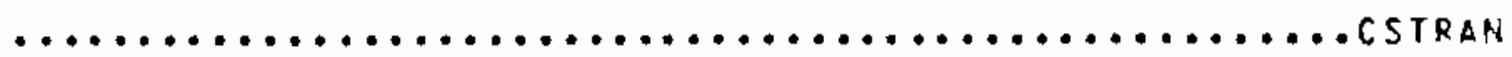

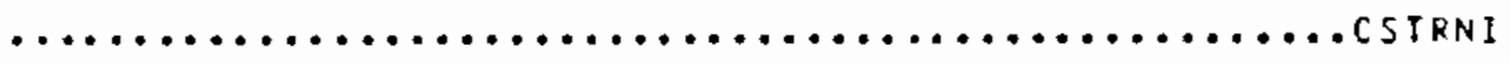

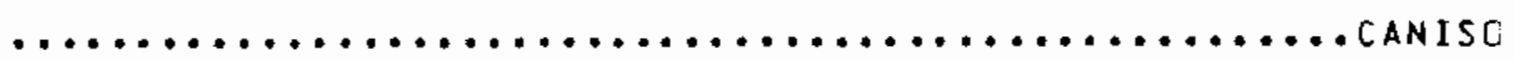

7. Elastic Modulus.......................................

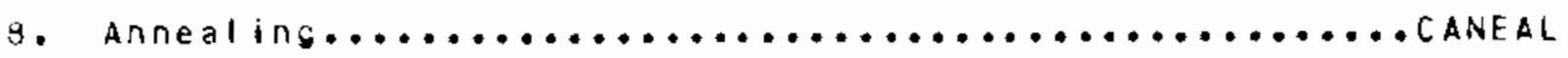

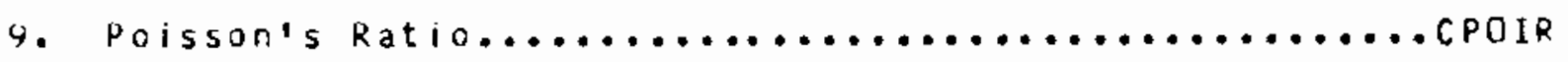

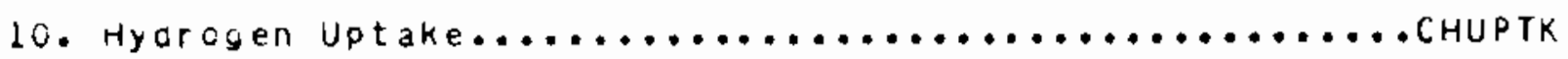

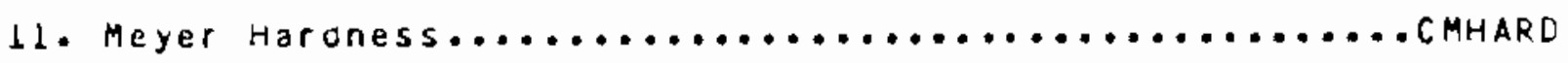

Gas and tuel kod Material Properties

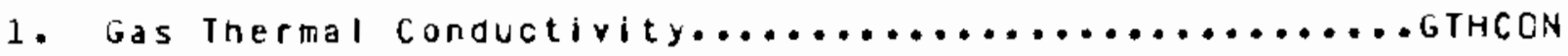

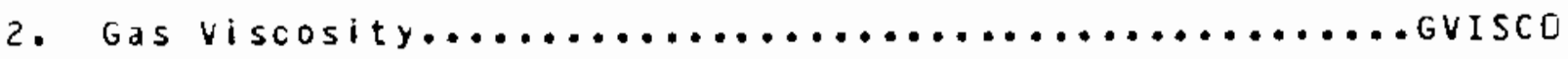

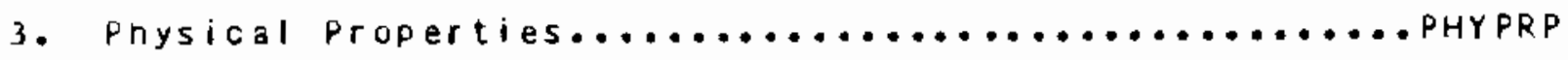


APPENEIXC

SUBKUUTINE RELATIONS TO MUDELS 
The information contained in appendix $C$ orovides the user with assistance in relating subroutine names with the function performed. Figure $C-1$ presents the FRAPCON-2 suoroutine relations and heirarchy.

1. Interaction of Subroutine RADIAL with ither Subroutines

As noted in Section 2.4.2, subroutine RADIAL takes fuel temperatures ana fuel/cladoing dimensions as input ano produces values at ach axial node for the fuel thermal conductivity cegradation. effective jap size, and effective fuel elastic moduli. Figure c- 2 lists these items and the fORTRAN variable name, and shows to which subroutine they go.

As shown, the fuel-ctagoing gap size (DG) and the fuel-cladoing interfacial pressure (PFG) to the gap conductance calculation in subroutine GAPRS. The interfacial pressure additionally goes to the mecinanical interaction calculation in the PtLET subcode to subroutine Asulve. The fuel radial and axial effective elastic moduli come into PELET to the subroutines STIFF ano STAFXL.

The degrading factor on the fuel thermal conouctivity (CFAC) goes directly to the fuel pellet radial temperature calculator (ODLF) where it modifies both the MATPRO conductivity and its oerivative with respect to temperature.

2. Interrelationship of subroutines in the PELET Subcode 

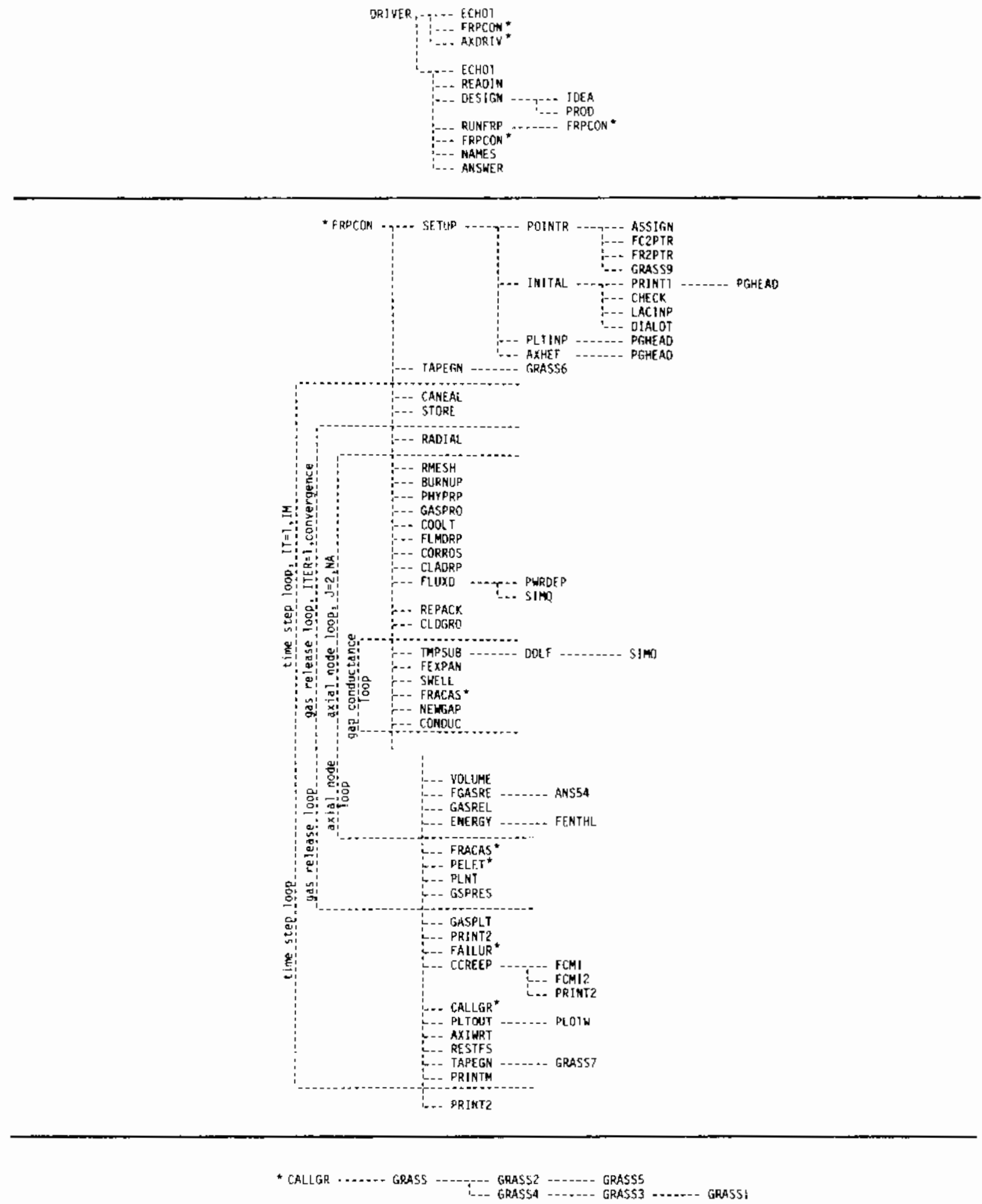

Fig. C-1 FRAPCON-2 subroutine relation and heirarchy. 


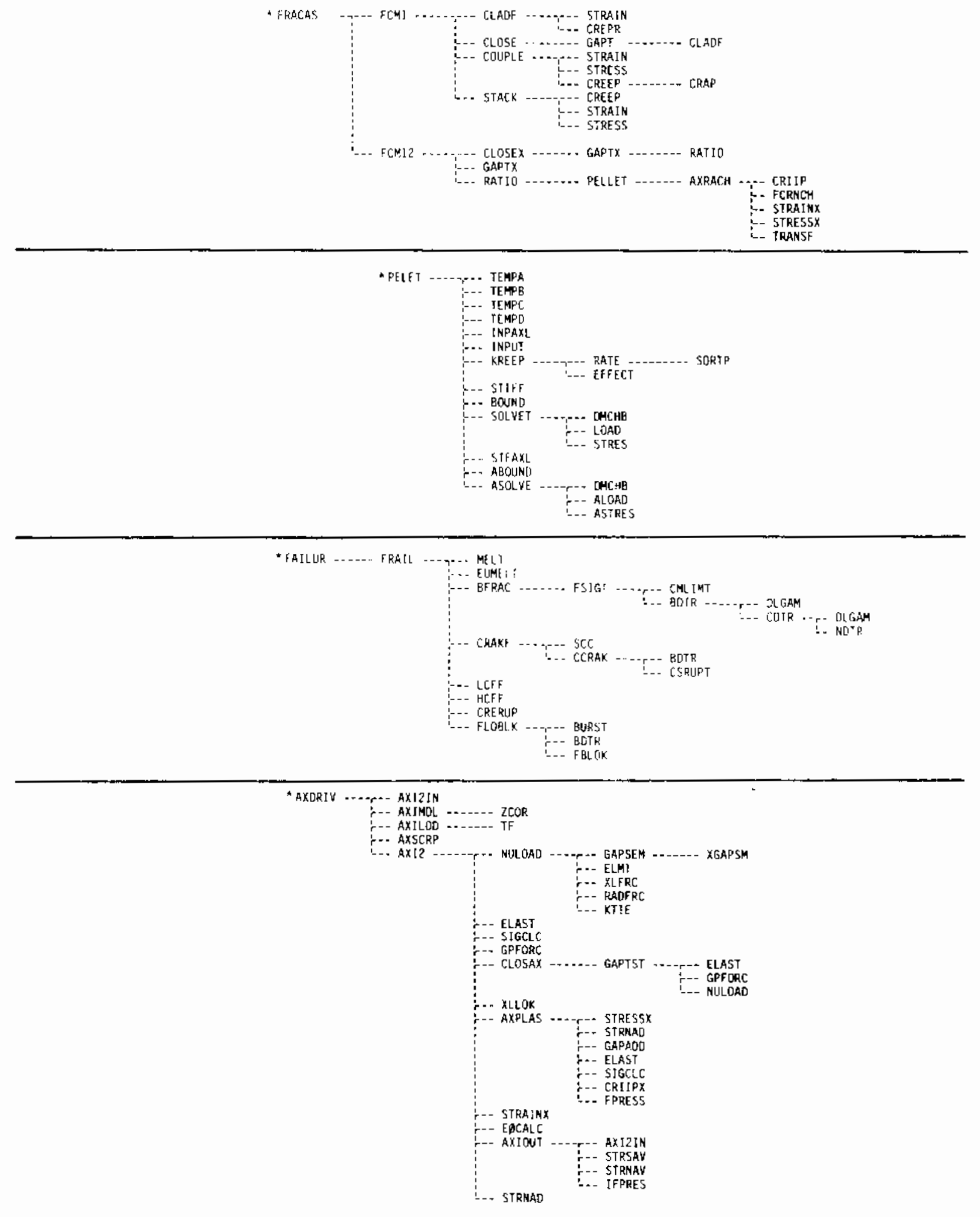

Figure C-1 (Cont.) FRAPCON-2 subroutine relation and
heirarchy. 


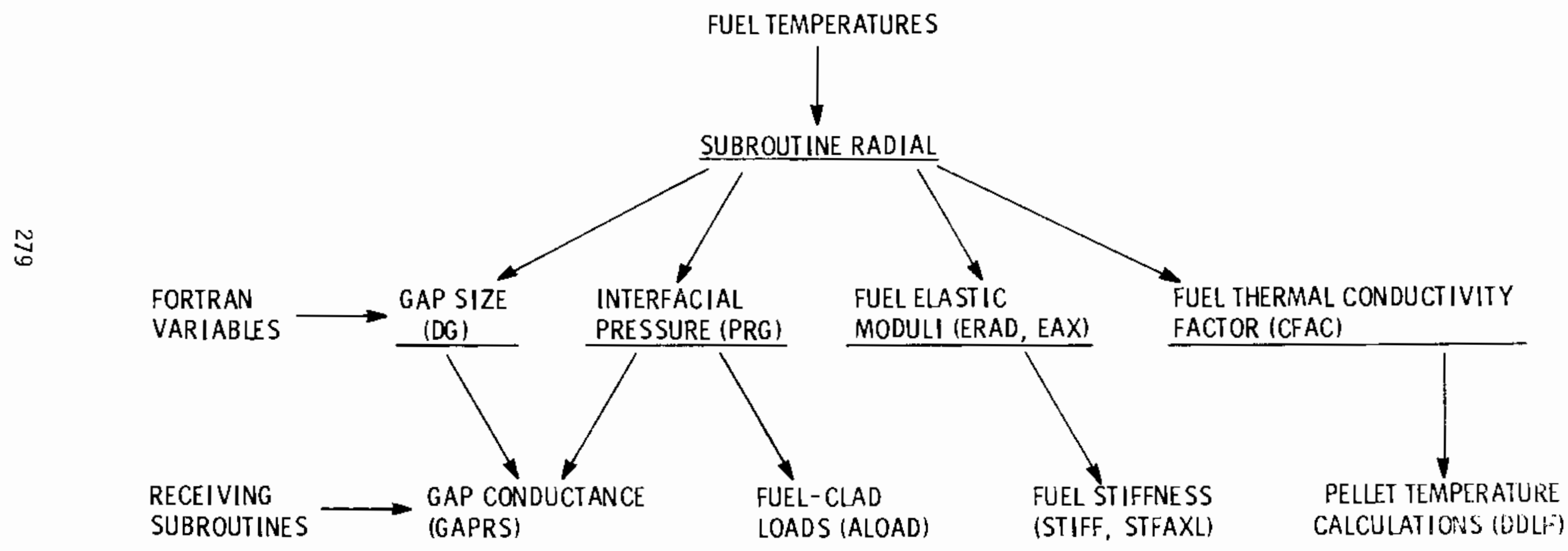

Figure C-2. Relation of subroutine RADIAL to other subroutines. 


\begin{tabular}{|c|c|c|}
\hline & Radial & Axial \\
\hline Function & $\begin{array}{l}\text { Model } \\
\text { Subroutine }\end{array}$ & $\begin{array}{l}\text { Model } \\
\text { Subroutine }\end{array}$ \\
\hline $\begin{array}{l}\text { Set up elerent ana global } \\
\text { stiffness matrix }\end{array}$ & STIFF & STFAXL \\
\hline $\begin{array}{l}\text { Apply Doundary conditions } \\
\text { ano constraints }\end{array}$ & BCUND & $A B O U N D$ \\
\hline Calculate ncaal point loads & $\angle O A O$ & $A L C A O$ \\
\hline Coordinate solution & SOLVET & ASOLVE \\
\hline $\begin{array}{l}\text { Perform solution of loao- } \\
\text { displacement equations }\end{array}$ & OMCHB & $\mathrm{DMCHB}$ \\
\hline $\begin{array}{l}\text { Calculate incremental and } \\
\text { total stresses }\end{array}$ & STRES & ASTRES \\
\hline
\end{tabular}

Note that the entire KKA loop is repeated for each creep sutstep, the difference being that the CREEP-EFFECT-RATE subroutine series is called first to provioe creep displacenents and a translation af these to initial strains, which are treated witnin alOAD/LQAD just like thermat strains. 
The Pinl fuel cladding mechanical interaction finite element calculation is performedin the pelet subcode, which is a collection of over 20 sudroutines. PELET calculates incremental stresses and strains in fuel and cladoing each time step, and accrues these in total stress/strain arrays. In this section we will show how the PELEJ sutroutines relate to one another. flow chart shoming the basis logic of PELET and the heirarchy of subroutines is jiven in rigure $\mathrm{C}-3$.

PelET first calls INPUT and INPAXL (once per case) to set wp the nade anc degree of freedom numbering sequences for the $r$ adial and axial nooels, respectively. PELET then calls TEMPA, TEMPB, TGEMPC and TEMPU to calculace current temperatures to be assigned to each of the finite elentents.

Then the elastic/plastic NKN loop begins. For NKN=1, elastic incremental calculations are performed; for $N K N=2$, creep and, if necessary, plastic deformations ano corresponding stress corrections are calculated. A subloop (the ITI loop) is executed for NKN=2 only if the creep time step must te suboivided to accomplist the calculation.

witnin the NKM loop resides the axial/radial loop (the KKA loop) mich coordinates the radiallaxial chained calculation. For KkAzl, the axial model calculation is performed; for KKA=2, the radial mooels representing each axial nooe are executed.

within the KKA loop resides the axial region loop (KA loop) which runs through all the axial regions of the rod. Within this loop reside the calls to radial-mocel subroutines. The function of the various suoroutines within the axial and radial models are listed DeION. 


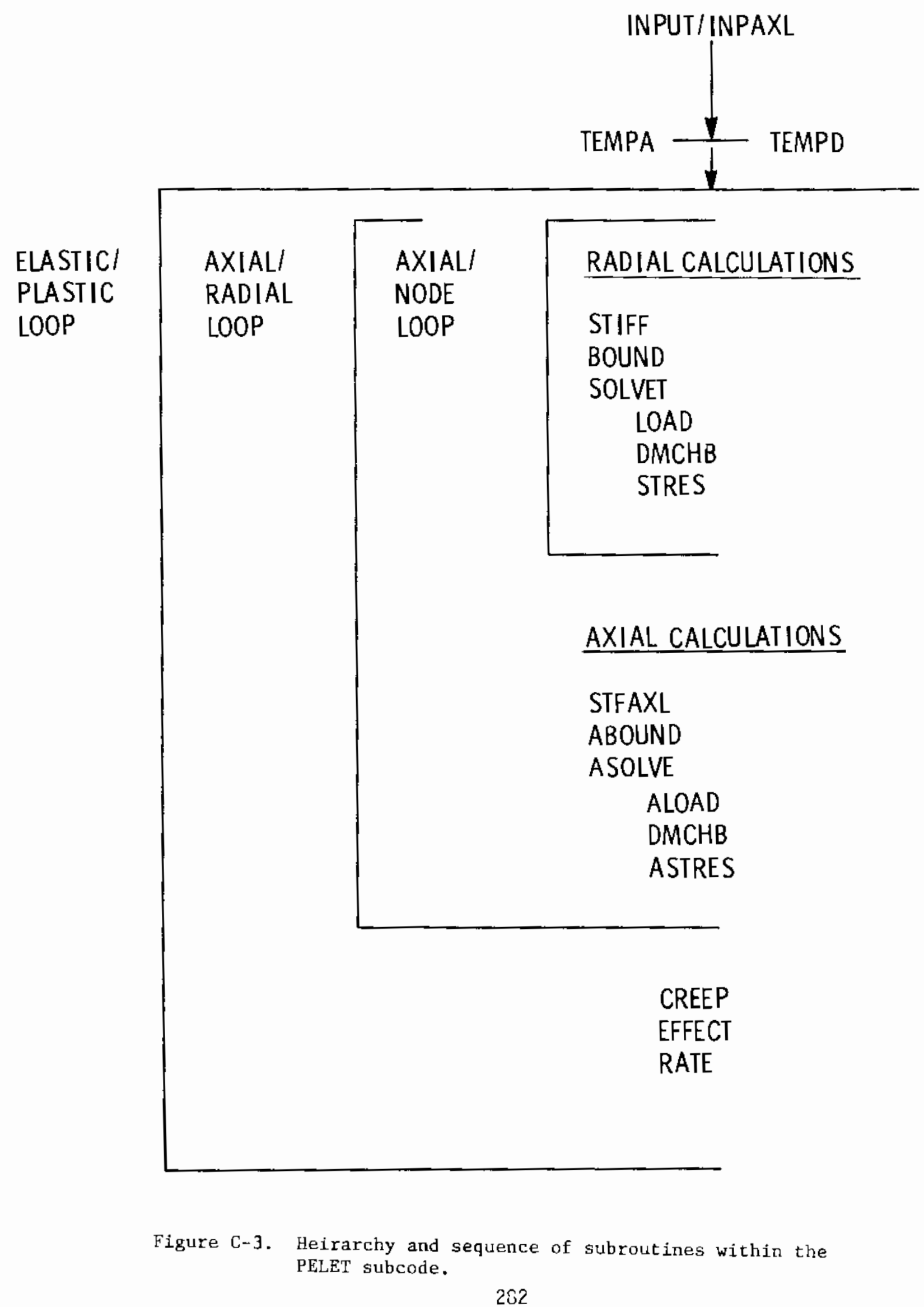




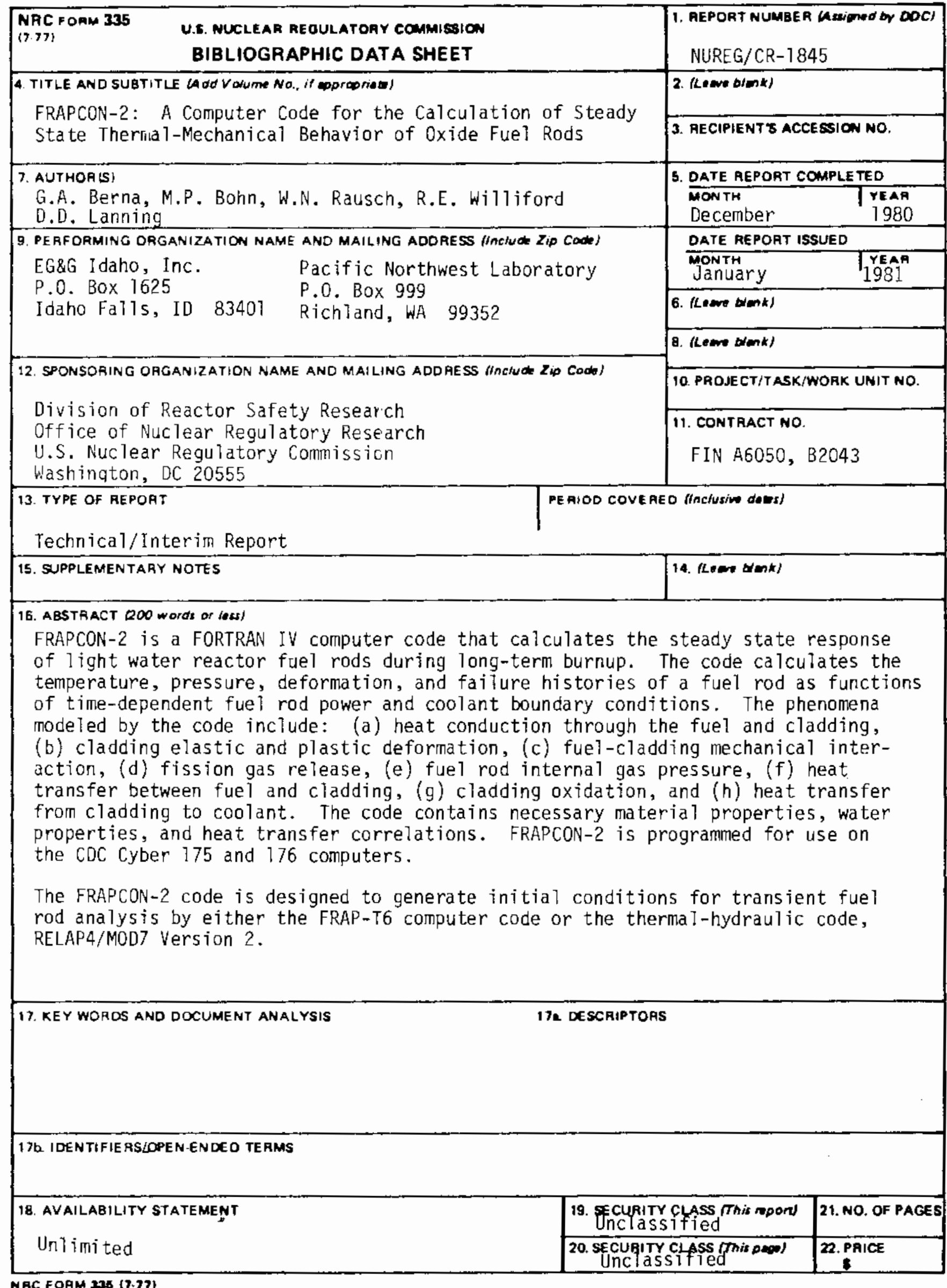


